University of South Florida

DIGITAL COMMONS

@ UNIVERSITY OF SOUTH FLORIDA
Digital Commons @ University of

South Florida

$4-1-2004$

\title{
Worksite Trip Reduction Model and Manual
}

CUTR

Follow this and additional works at: https://digitalcommons.usf.edu/cutr_nctr

\section{Scholar Commons Citation}

CUTR, "Worksite Trip Reduction Model and Manual" (2004). Research Reports. 215.

https://digitalcommons.usf.edu/cutr_nctr/215

This Technical Report is brought to you for free and open access by the National Center for Transit Research (NCTR) Archive (2000-2020) at Digital Commons @ University of South Florida. It has been accepted for inclusion in Research Reports by an authorized administrator of Digital Commons @ University of South Florida. For more information, please contact digitalcommons@usf.edu. 


\title{
WORKSITE TRIP REDUCTION MODEL AND MANUAL
}

\author{
April 2004 \\ Prepared for \\ Florida Department of Transportation \\ BC-137-41 \\ Prepared by \\ National Center for Transit Research \\ Center for Urban Transportation Research \\ University of South Florida \\ 4202 E. Fowler Avenue, CUT100 \\ Tampa, FL 33620-5375 \\ (813) $974-3120$ \\ http://www.nctr.usf.edu/
}

Florida Department of Transportation Project Manager:

Michael Wright, Commuter Assistance Program Manager

Principal Investigators:

Philip L. Winters, TDM Program Director, CUTR

Rafael A. Perez, Computer Science \& Engineering, USF

Project Staff:

J ennifer Perone, Research Associate, CUTR

Ajay J oshi, Graduate Research Assistant, Computer Science \& Engineering

Aaron Gregory, Graduate Research Assistant, Computer Science \& Engineering

J onathan Hershberger, Grad. Research Assistant, Computer Science \& Engineering

The opinions, findings and conclusions expressed in this publication are those of

the authors and not necessarily those of the State of Florida Department of Transportation, or the US Department of Transportation.

Prepared in cooperation with the State of Florida Department of Transportation, or the US Department of Transportation 


\begin{tabular}{|c|c|c|}
\hline $\begin{array}{l}\text { 1. Report No. } \\
\text { NCTR-473-14, FDOTBC137 } \\
\text { RPWO\#41 }\end{array}$ & 2. Government Accession No. & 3. Recipient's Catalog No. \\
\hline \multirow{2}{*}{\multicolumn{2}{|c|}{$\begin{array}{l}\text { 4. Title and Subtitle } \\
\text { Worksite Trip Reduction Model and Manual }\end{array}$}} & $\begin{array}{l}\text { 5. Report Date } \\
\text { April } 2004\end{array}$ \\
\hline & & 6. Performing Organization Code \\
\hline $\begin{array}{l}\text { 7. Author(s) } \\
\text { Winters, Philip L.; Perez, Rafael A.; Peror }\end{array}$ & e, Jennifer S.; \& Joshi, Ajay. & 8. Performing Organization Report No. \\
\hline \multirow{2}{*}{\multicolumn{2}{|c|}{$\begin{array}{l}\text { 9. Performing Organization Name and Address } \\
\text { Center for Urban Transportation Research } \\
4202 \text { E. Fowler Avenue, CUT } 100 \\
\text { Tampa, FL } 33620-57350\end{array}$}} & 10. Work Unit No. \\
\hline & & $\begin{array}{l}\text { 11. Contract or Grant No. } \\
\text { DTRS98-G-00329 }\end{array}$ \\
\hline \multirow[t]{2}{*}{$\begin{array}{l}\text { 12. Sponsoring Agency Name and Address } \\
\text { Office of Research and Special Programs } \\
\text { U.S. Department of Transportation } \\
\text { Washington, DC } 20590\end{array}$} & $\begin{array}{l}\text { Florida DOT } \\
605 \text { Suwannee } \\
\text { Tallahassee, Florida } 32399\end{array}$ & 13. Type of Report and Period Covered \\
\hline & & 14. Sponsoring Agency Code \\
\hline
\end{tabular}

15. Supplementary Notes

Supported by a Grant from the USDOT Research and Special Programs Administration, and the Florida Department of Transportation.

16. Abstract

According to Institute of Transportation Engineers, assessing the trip reduction claims from transportation demand management (TDM) programs is an issue for estimating future traffic volumes from trip generation data. To help assess those claims, a Worksite Trip Reduction Model and Manual was produced using existing data on programs, services and incentives contained in thousands of before and after worksite trip reduction plans. Models were built using linear regression and non-linear neural networks with the change in vehicle trip rate (VTR) as the dependent variable. No single variable selection technique, data handling method, or modeling approach yielded the best-fitting model for all three urban areas. The neural network model built on equally sampled data was the best generalized model based on three performance measures: the accuracy across the moderate range of change in VTR; the accuracy on full range of change in VTR and the R-square between the actual change in VTR and the predicted change in VTR. Worksite trip reduction plans explain a modest portion of the change in vehicle trip rates from one year to the next. The smaller datasets may have affected neural networks ability to identify correct non-linear relationship by overfitting the training data and reducing the neural network model's power to generalize over unseen validation data. The aggregate nature of the data loses the ability to explain whether the change in mode behavior was influenced by the programs or changes in the workforce or other exogenous variables. Quality control issues with the provided datasets affected the model building process. Future research should examine organizational culture, management styles, the total expenses incurred by the employer, employee demographics and changes in the local economy. Finally, this Worksite Trip Reduction Model and Manual should be allowed to evolve like the ITE's Trip Generation Manual, which is in its sixth edition. Efforts to improve, maintain, and disseminate such a document are critical to its widespread application and improved understanding about transportation demand management program effects on traffic volumes and parking demands.

17. Key Words

Transportation Demand Management, TDM, trip reduction, Carpool, Vanpool Telecommuting, Transit, Compressed Work Weeks, Flextime, Neural Network, Trip Generation

19. Security Classif. (of this report) Unclassified
18. Distribution Statement

Available to the public through the National Technical Information Service (NTIS), 5285 Port Royal Road, Springfield, VA 22161, (703) 487-4650, http://www.ntis.gov/, and through the NCTR web site at http://www.nctr.usf.edu/.

20. Security Classif. (of this page)

Unclassified
21. No. of pages
139 


\section{Acknowledgements}

The research team acknowledges the cooperation and support of many individuals, including FDOT project manager, Michael Wright. In addition, we would like to extend our sincere thanks to Ed Hillsman and Brian Lagerberg with the Washington State Department of Transportation, Rita Hildebrand with the Pima Association of Governments, and Antonio Thomas and Carol Gomez with the South Coast Air Quality Management District for providing the data and helping explain the nuances. 


\section{TABLE OF CONTENTS}

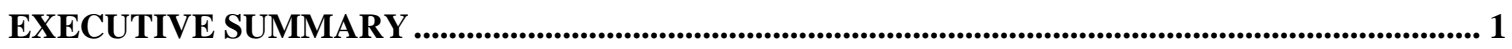

I. INTRODUCTION...................................................................................................................... 6

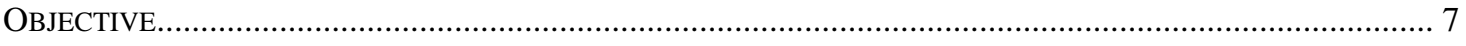

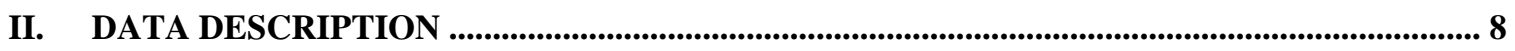

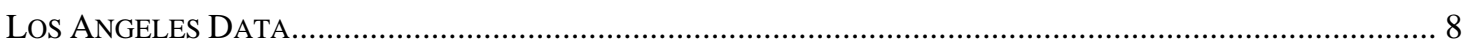

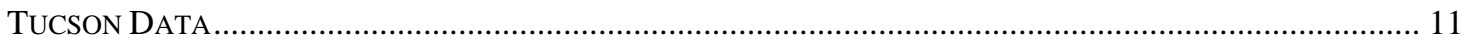

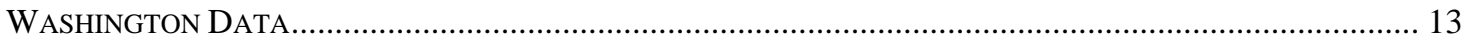

III. MODEL BUILDING APPROACHES ...................................................................................... 16

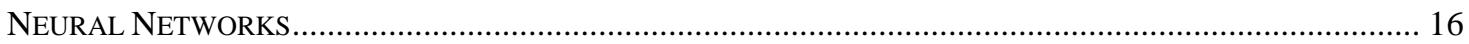

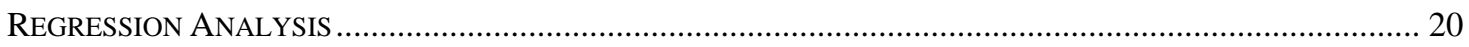

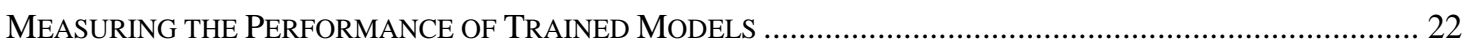

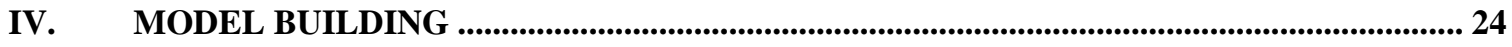

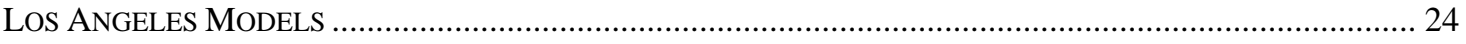

Phase I: Los Angeles Full Sample Data ................................................................................ 24

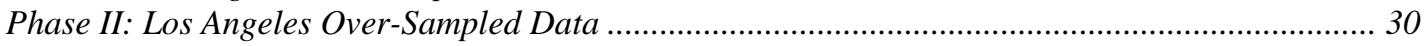

Phase III: Los Angeles Data - records with no incentives removed ..................................................... 33

Phase IV: Los Angeles Data - records with no incentives \& outliers removed ................................... 38

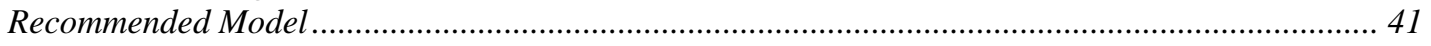

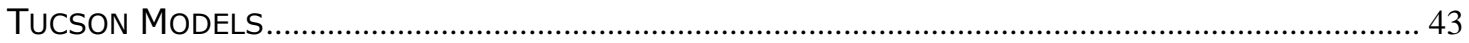

Phase I: Tucson Full Sample Data ................................................................................................ 43

Phase II: Tucson Over-Sampled Data ................................................................................. 52

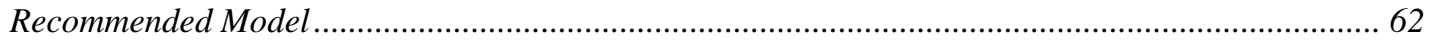

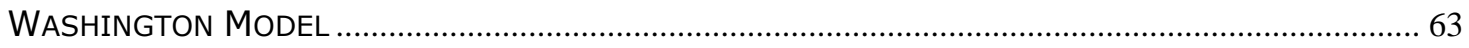

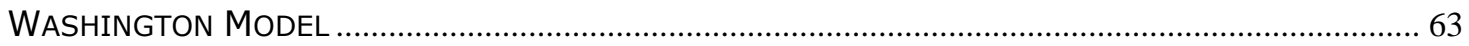

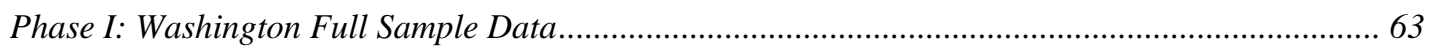

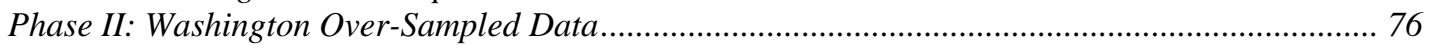

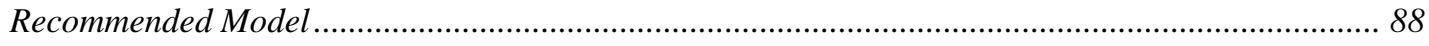

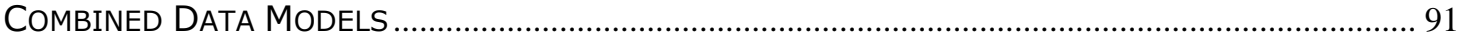

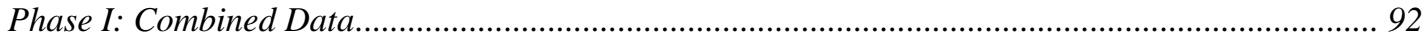

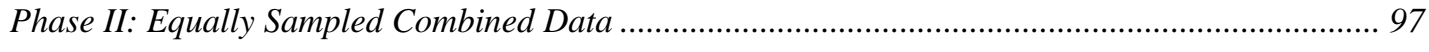

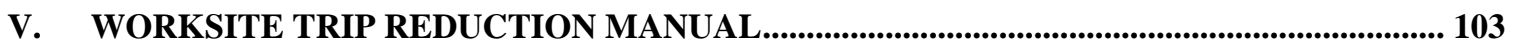

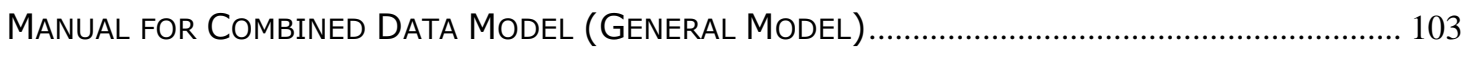

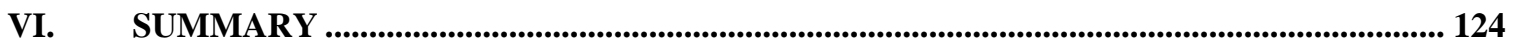

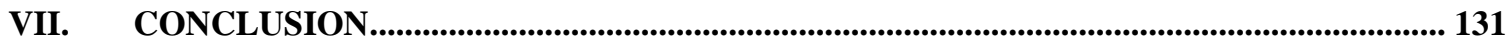

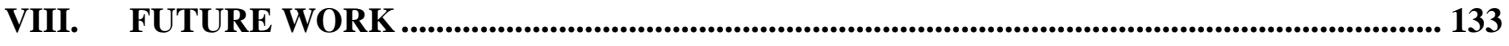




\section{List of Tables}

Table 1: Los Angeles Data variable description and grouping $\ldots \ldots \ldots \ldots \ldots \ldots \ldots \ldots \ldots$

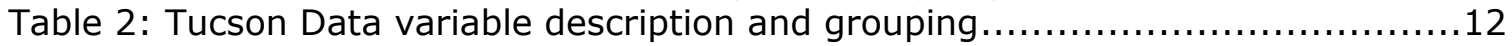

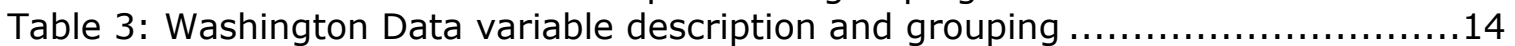

Table 4: Los Angeles Full Sample Data - No. of Records in bins ........................

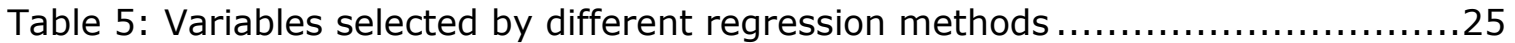

Table 6: Detailed accuracies for NN model on stepwise regression variables ..........28

Table 7: Los Angeles Over-Sampled Data - No. of Records in bins ..................... 30

Table 8: Los Angeles data with records with no Incentives Removed - No. of Records

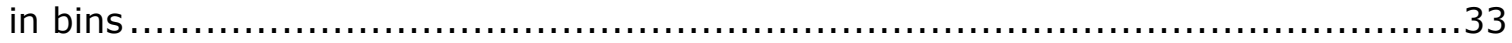

Table 9: Variables stepwise regression--data with associated incentive costs .........33

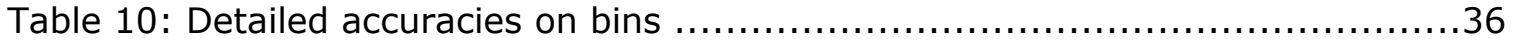

Table 11: Los Angeles data with records no incentives \& outliers removed- - No. of

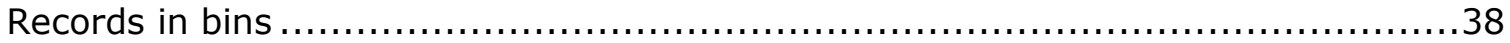

Table 12: Variables for recommended model Los Angeles Model .....................42

Table 13: Tucson Full Sample Data - No. of Records in bins .........................43

Table 14: Variables selected by different models on Full Sample data ................45

Table 15: Detailed accuracies for neural net model with no variable selection ........46

Table 16: Variables with grouped incentives.......................................... 47

Table 17: Detailed accuracies for forced enter regression model .....................49

Table 18: Variables and the associated forced enter regression coefficients ...........51

Table 19: Tucson Over-Sampled Data - No. of Records in bins ....................... 52

Table 20: Variables selected by different models on Tucson over-sampled data.....54

Table 21: Detailed accuracies on bins: Tucson over-sampled data. ....................55

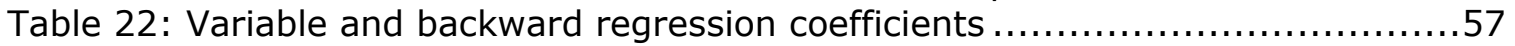

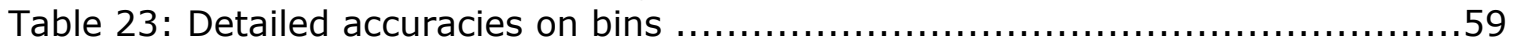

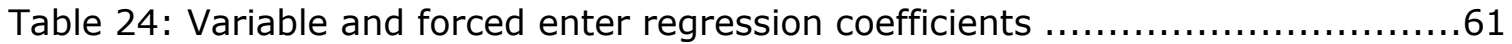

Table 25: Washington Full Sample Data - No. of Records in bins.......................63

Table 26: Variables selected by different models on Full Sample data ...................66

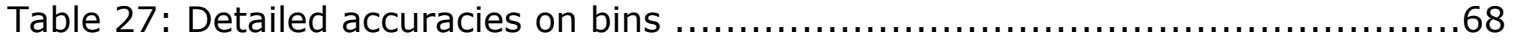

Table 28: Variables and coefficients for forced enter regression model ................69

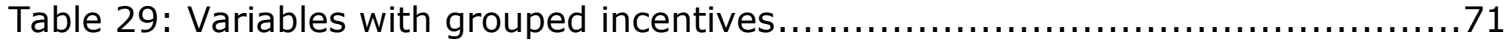

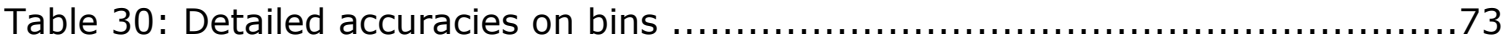

Table 31: Variables and coefficients for forced enter regression model ...............75

Table 32: Washington Over-Sampled Data - No. of Records in bins ....................76

Table 33: Variables selected by different models on over-sampled data ...............78

Table 34: Detailed accuracies on bins .............................................. 80

Table 35: Variables and coefficients for forced enter regression model ...............82

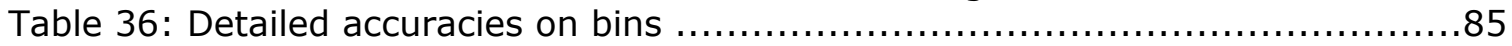

Table 37: Variables and coefficients for forced enter regression model ................87

Table 38 A-D: Validation Set......................................................... 88

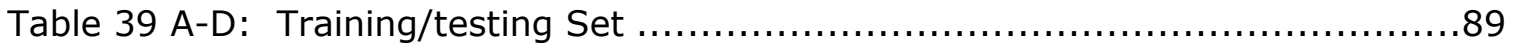

Table 40: Variable mapping for combining all three datasets $\ldots \ldots \ldots \ldots \ldots \ldots \ldots \ldots \ldots 1$

Table 41: Combined Data - No. of Records in bins ................................... 92

Table 42: Equalized Combined Data - No. of Records in bins $\ldots \ldots \ldots \ldots \ldots \ldots \ldots \ldots \ldots \ldots \ldots$

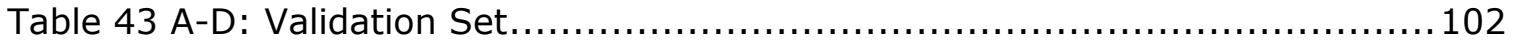

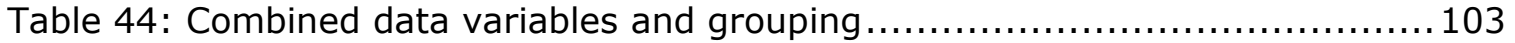

Table 45: Widely implemented incentive plan combinations.......................... 106

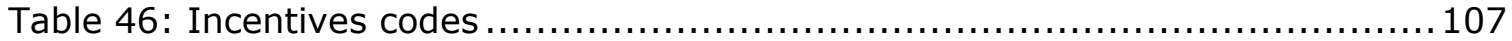

Table 47: Model's change in VTR prediction for worksites with Transit share between

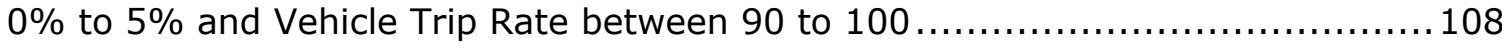


Table 48: Model's change in VTR prediction for worksites with Transit share between $0 \%$ to $5 \%$ and Vehicle Trip Rate between 80 to 90.

Table 49: Model's change in VTR prediction for worksites with Transit share between $0 \%$ to $5 \%$ and Vehicle Trip Rate between 70 to 80.

Table 50: Model's change in VTR prediction for worksites with Transit share between

$0 \%$ to $5 \%$ and Vehicle Trip Rate between 60 to $70 . .$.

Table 51: Model's change in VTR prediction for worksites with Transit share between

$0 \%$ to $5 \%$ and Vehicle Trip Rate between 50 to 60.

Table 52: Model's change in VTR prediction for worksites with Transit share between

$5 \%$ to $15 \%$ and Vehicle Trip Rate between 90 to 100 ...

Table 53: Model's change in VTR prediction for worksites with Transit share between

$5 \%$ to $15 \%$ and Vehicle Trip Rate between 80 to 90 114

Table 54: Model's change in VTR prediction for worksites with Transit share between

$5 \%$ to $15 \%$ and Vehicle Trip Rate between 70 to 80

Table 55: Model's change in VTR prediction for worksites with Transit share between

$5 \%$ to $15 \%$ and Vehicle Trip Rate between 60 to 70

Table 56: Model's change in VTR prediction for worksites with Transit share between

$5 \%$ to $15 \%$ and Vehicle Trip Rate between 50 to 60

Table 57: Model's change in VTR prediction for worksites with Transit share between

$15 \%$ to $25 \%$ and Vehicle Trip Rate between 70 to 80

Table 58: Model's change in VTR prediction for worksites with Transit share between

$15 \%$ to $25 \%$ and Vehicle Trip Rate between 60 to 70

Table 59: Model's change in VTR prediction for worksites with Transit share between

$15 \%$ to $25 \%$ and Vehicle Trip Rate between 50 to 60

Table 60: Model's change in VTR prediction for worksites with Transit share between

$25 \%$ to $35 \%$ and Vehicle Trip Rate between 60 to 70

Table 61: Model's change in VTR prediction for worksites with Transit share between

$25 \%$ to $35 \%$ and Vehicle Trip Rate between 50 to 60

Table 62: Model's change in VTR prediction for worksites with Transit share between

$25 \%$ to $35 \%$ and Vehicle Trip Rate between 50 to 60

Table 63 Performance Measures for Best Generalized Model 


\section{EXECUTI Ve SUMMARY}

Today's transportation professionals and others often use Institute of Transportation Engineers' (ITE) Trip Generation Manual and the Parking Generation Manual for estimating future traffic volumes to base off-site transportation improvements or identifying parking requirements. Planners may use these same resources to evaluate the impacts of land use or zoning changes on the transportation system. According to ITE, one of the many issues facing users of this trip generation data is assessing the claims that "specific transportation demand management programs and transit services will reduce site trip generation by a certain amount." This research project was undertaken to help transportation planners and engineers assess those claims.

Using literally thousands of before/after trip reduction plans from employers with 100 or more workers, a variety of analytical techniques were applied to predict the change in vehicle trips due to worksite demand management programs and policies. The products of the effort are this Worksite Trip Reduction Manual and web-based model.

The Worksite Trip Reduction Model (WTRM) (http://www.nctr.usf.edu/worksite/) predicts the extent that each incentive, disincentive, or program would impact traffic volumes and parking needs in a specific worksite. This model would allow transportation engineers, local planners, developers, employers, and transportation demand management professionals to easily input various programs, incentives, disincentives, and worksite characteristics to obtain predictions about the change in vehicle trips from that mix of tactics.

The development of this manual and model will also save those stakeholders time and money. These tools will allow for a quick assessment of different worksite-based transportation demand management strategies on traffic volumes and parking impacts. WTRM could be used to assess parking needs of new developments, thereby reducing the cost of parking construction. Employers and developers would also know what types of services and programs to offer employees, residents and tenants to decrease onsite traffic congestion and reduce their need for parking. And, finally, reduction in vehicle traffic and vehicle miles traveled will improve air quality in the region.

\section{Methodology}

This project used several thousand worksite trip reduction plans to build the model. The data came from three urban areas in the United States: Los Angeles, Tucson, and Washington State that have had trip reduction requirements on employers for many years. Employers were required to submit plans to reach a particular objective such as a reduction in the 
levels of single occupant vehicle (SOV) use. The data consisted of worksite modal characteristics aggregated at the employer level and a listing of incentives and amenities offered by employers. The Los Angeles data contained the largest data set data, with the Tucson, Arizona and Washington state data sets being considerably smaller. Data quality control problems reduced the size of the data in each area and eliminated or restricted some potentially useful variables (e.g., dollar values of some incentives). For performance evaluation the datasets were divided in two disjoint sets 'training/testing set' which was used to build the models and 'validation set' which was used as an unseen data to evaluated the models.

The dependent variable chosen was the change in vehicle trip rate (VTR) (e.g., reduction of 4.5 vehicles per 100 employees). VTR correlates closely with the goals of TDM -- reduce trips, decrease air pollution, decrease the need for parking -- and is generally proportional to the desired result. Alternative dependent variables such as SOV share or average vehicle ridership (AVR) have disadvantages. SOV share misses the benefits of moving from one non-SOV mode to another where the switch may actual reducing traffic but not affect SOV share (e.g., carpool to transit). The reduction in vehicle trips is distorted when using AVR as the dependent variable due to the non-liner relationship between AVR and vehicle trips. For example, increasing AVR by 0.25 from 1.10 to 1.35 persons per vehicle would require a reduction in 17 vehicle trips per 100 people. The same increase (0.25) for a worksite with an AVR of 1.50 to 1.75 would only require a reduction in 9 vehicle trips per 100 people.

Two approaches were used for the model building process: linear statistical regression models and non-linear neural networks. The linear statistical regression models were used as a benchmark for the validity and accuracy of the neural net models. The linear statistical regression models minimize the sum of the error between the real and predicted data, learning simple linear relationships between the worksite characteristics, incentives and the dependant variable 'change in VTR', while the neural networks learn more complex non-linear relationships. Sometimes linear regression methods were used to determine which variables the neural net would use to build its models.

Several phases were followed to build the models. Models were built for each of the three datasets using a variety of approaches of handling the data, including variable selection, grouping of incentives, and the treatment of outliers. Models were also built after combining the data from the three urban areas into a single dataset. Under the assumption the transportation industry was most interested in a model that predicted when large reductions could be achieved, the model performance objective was focused on predicting the change equally well across the range of the changes in VTR. 


\section{Results AND Conclusi ons}

No single variable selection technique, data handling method, or modeling approach yielded the best-fitting model for all urban areas. In many cases, there was no significant performance difference between the top models, so the recommended model for some dataset had to be decided by using the F-value measure, which incorporated two other metrics: Recall which gave a measure of the completeness of the model, and Precision which gave a measure of the correctness of the model.

The best model for each city also was not the model that used data only from that city. Before combining the Los Angeles data set with those from the other two areas, the preferred model was the one built on the grouped incentives data with records with 'no incentives' removed. But after combining the datasets, the neural network model built with no variable selection performed better for Los Angeles than the model built with only data from Los Angeles. Also for Tucson data, a neural network model built on the equally sampled (i.e., each dataset contributes equally) data performed better than the previously selected neural network model built on the full sample (i.e., all valid records from the dataset) with ungrouped incentives data. The best model for the Washington data was the linear forced enter regression model built on full sample with ungrouped incentive data.

The generalized models for any urban area were built on the combined training datasets and equally sampled training datasets. The models built using equally sampled datasets were the ones which were not biased towards the any dataset. Overall, the best generalized model for any location is the neural net model built on equally sampled data based on three performance measures. The first performance measure is the accuracy across the moderate range of change in VTR. The second performance measure is the accuracy on full range of change in VTR. The third measure is the R-square between the actual Delta_VTR and the Predicted Delta_VTR. This is the model at http://www.nctr.usf.edu/worksite.

Overall, the neural net models performed better than the linear regression models. This might be due to the ability of the neural network program to move beyond simple linear regression, which tries to minimize the error between the predicted and actual data sets. The neural network models in many situations were able to learn the non-linear relationships among various combinations of strategies. There were some neural network models which performed worse than the linear models. This might be due to the over-fitting of the training data and reducing the neural net's power to generalize over unseen validation data.

Quality control issues with the provided datasets affected the model building process. In the case of the Los Angeles data, there were many worksites for which some incentives were available in one year, then not a 
single incentive was shown in the following year, only to have incentives "reappear" the next plan cycle. These unexplained gaps in reporting can affect the ability of the model to estimate the impact of a particular incentive.

The quality of the financial data and the limited information on the levels of financial support provided by employers for individual employees hindered the use of such data that may have helped explain more of the changes. Not every community asked for the dollar value of transit subsidies, for example.

Another data problem encountered was the use of different units of measurement across programs. For example, the transit subsidy values were reported ranging from $\$ 0.20$ to $\$ 3,000$. Though employers were to report these values as "cost per employee per month", the lower figure basis appears to be along the lines of "cost per employee per trip" and the upper figure might be the "total cost per employee using the mode per year". The difficulty is there is no way of telling from the data. Also it was found that the incentives had differences in their definition across different datasets which introduced error into the results. This problem made modelbuilding a complex task when trying to condense and collapse all of the similar variables into one.

The aggregate nature of the data loses the ability to explain whether the change in mode behavior was influenced by the programs or changes in the workforce or other exogenous variables. While hundreds of thousands of employee data, including employee's preferences to particular options, were available from the State of Washington dataset, there was no identifier to track individual changes in behavior over time. The other two datasets did not have any comparable data to Washington's individual survey responses. Access to such disaggregate data could improve the ability of a model to track behavior changes over time based on changes to worksite incentives, amenities and programs.

\section{FUTURE WORK}

Given the common interest shared by the public sector and worksites in assessing the relative effectiveness of worksite trip reduction program, future work should begin by improving the quality of the data already being collected. Quality can be improved by adopting standard definitions and common terminology to make more use of the data collected by others to improve the accuracy of the model under different circumstances. Common terms will contribute to an expanded dataset by making the data compatible with other data from other parts of the country. This approach could be facilitated by the creation of a centralized database.

Adhering to quality control procedures also could add more explanatory power to the data, especially as it relates to the value of financial 
incentives. Improving the quality of information that already exists can help worksites more cost-effectively deliver vehicle trip reduction programs.

The data used in this model-building approach was aggregated to the worksite level prior by the employer. This aggregate level detail does not allow for analysis to determine individual changes in travel behavior. In order to get a real handle on what makes VTR increase or decrease and what causes people to choose alternative transportation options, attempts to control for the differences should be used. Access to disaggregate data collected over time can help establish a "test" and "control" group approach to control for differences, for example, in the composition of the workforce.

Access to the disaggregate data also would help track the long-term effects of the programs. The current project assesses the impacts between two time periods (usually separated by only one to two years). However, the cumulative effect of these programs over time is less understood (i.e., will the worksite experience a constant, variable, or exponential change in VTR over time as the programs diffuse within the workforce and move beyond the "early adopters"?) Diffusion is the process by which the trip reduction program offerings are communicated through certain channels over time among the employees at that location. While the collection of individual data may be difficult due to privacy and attrition issues, it is worth investigating the possibility of collecting this type of transportation behavior data to help develop sustainable transportation strategies and programs for the future.

Worksite trip reduction plans explain a modest portion of the change in vehicle trip rates from one year to the next. Future research should build on this research by examining other factors that could explain more of the variance. Organizational culture and management styles are two factors hypothesized as having a significant effect on performance. These factors may affect management support at all levels, including the selection and support for the organization's employee transportation coordinator (ETC). ETCs may have the responsibility but not the authority or resources to carry out an effective program. Turnover at the ETC position can also affect continuity of service delivery and the quality of the effort. Other factors such as the total expenses incurred by the employer, employee demographics and changes in the local economy also should be examined.

Finally, this Worksite Trip Reduction Model and Manual should be allowed to evolve like the ITE's Trip Generation Manual, which is in its sixth edition. Modest levels of financial and technical assistance efforts will be needed to make this happen. Efforts to improve, maintain, and disseminate such a document are critical to its widespread application and improved understanding about transportation demand management program effects on traffic volumes and parking demands. 


\section{I NTRODUCTION}

For years, transportation engineers and urban planners have relied on the Institute of Transportation Engineers' Trip Generation Manual and the Parking Generation Manual to estimate the traffic impact and parking needs of new developments. The trip generation estimates predict the traffic volumes of these development proposals and decisions regarding whether to allow new developments are tied to these estimates. The link between development and available capacity is especially important in areas with insufficient roadway "supply" to absorb the new development "demand" without degrading the level of service.

Both the ITE trip generation manual and the Parking Generation Manual are missing modifications to the demand side of the equation. According to ITE, one of the many issues facing users of this trip generation data is assessing the claims that "specific transportation demand management programs and transit services will reduce site trip generation by a certain amount."

This research project was undertaken to help transportation planners and engineers assess those claims. Using literally thousands of before/after trip reduction plans from employers with 100 or more workers, a variety of analytical techniques were applied to predict the change in vehicle trips due to worksite demand management programs and policies. The products of the effort are this Worksite Trip Reduction Manual and web-based model.

This project seeks to help these transportation professionals by compiling several thousand worksite trip reduction plans that have been developed and tracked for several years from three urban areas in the United States: Los Angeles, Tucson, and Washington State. These data sets were compiled into models of best fit via both Neural Net and regression model building with statistical software.

The model, VTR, predicts the extent that each incentive, disincentive, or program would impact traffic volumes and parking needs in a specific worksite. This model would allow transportation engineers, local planners, developers, employers, and transportation management associations to easily input various programs, incentives, and disincentives to obtain predictions about the change in vehicle trips from that mix of tactics.

Chapter 2 of this report summarizes the data from each of the three areas. Chapter 3 provides a description of the model building approaches. Chapter 4 provides the results of the models for each of the three areas, including the best model for each area as well as the model built with the combined and sampled datasets. Chapter 5 provides a series of lookup tables to 
complement the web-based model, VTR. Chapter 6 provides future research ideas. Chapter 7 provides a summary and Chapter 8 provides conclusions.

\section{OBJ ECTIVE}

To develop a Worksite Trip Reduction Model and a Manual that will estimate the impacts of various combinations of transportation demand management (TDM) strategies in reducing vehicle trips. 


\section{I. DATA DESCRI PTI ON}

The data used in this project was obtained from three different locations: Los Angeles, California; Tucson, Arizona; and several locations in the state of Washington. The following sections describe the available variables for each data set.

\section{Los ANGELES DATA}

The Los Angeles data was obtained from South Coast Air Quality Management District for Los Angeles and consisted of 33,092 total records from 7,626 company worksites. Each record represents information from a specific company worksite for a specific year. There can be several records from a specific worksite as well as several records for a company if that company has multiple worksites. The information in a record includes worksite characteristics such as shares of different modes of transportation used by employees for commuting, the different incentives used by the company to entice employees to use other commuting modes different from driving alone, etc. for a specific company. The names of incentive plans in the dataset followed a coding convention, so that the names with same first two letters were closely related. This knowledge was used in some cases to group several individual incentives into one. The reason for this was to avoid collinearity of the variables, or shared variance. By grouping similar variables together, it cuts down on this problem of shared variance and produces fewer variables with which less complex models can be built. These grouped variables also give more explanatory power to the modelbuilding process.

The incentive grouping and field descriptions for Los Angeles are shown in Table 1. Each record included a total of 95 fields. Several years of data was collected from 1988 to 2001. Some worksites collected data only for a subset of these years. The vehicle trip rate for each worksite was calculated from the given data using -

VTR $=100 *($ CAR $1+$ MOTORCYCLE + CAR2 $/ 2+$ CAR3 $/ 3+$ CAR4/4 + CAR5 $/ 5+$ CAR6/6 + VAN_CUTR/7) / (CAR1 + MOTORCYCLE + CAR2 + CAR3 + CAR4 + CAR5 + CAR6 + VAN_CUTR + BUS + TRANSIT + WALK + BIKE + TELECOMMUTE + CWW336 + CWW440 + CWW980)

Where,

CAR1 - Number of employees driving alone

MOTORCYCLE - Number of employees commuting by motorcycle

CAR2 - Number of employees commuting two together

CAR3 - Number of employees commuting three together

CAR4 - Number of employees commuting four together

CAR5 - Number of employees commuting five together

CAR6 - Number of employees commuting six together 
VAN_CUTR - Number of employees commuting in van

BUS - Number of employees commuting by bus

TRANSIT - Number of employees commuting using transit

WALK - Number of employees commuting walking

BIKE - Number of employees commuting by bike

TELECOMMUTE - Number of employees telecommuting

CWW336 - Number of $3 / 36$ days off

CWW440 - Number of 4/40 days off

CWW980 - Number of $9 / 80$ days off

Given that the objective was to build a model that could predict the effect on VTR of one or more incentives introduced by a worksite, the data described above was used to calculate the change in VTR ( $96^{\text {th }}$ field) between consecutive years. For example, worksite 'A' in 1999 had a VTR of 90 and in 2000 had a VTR of 85, then the difference in VTR (85 - 90) of -5 was associated with the 1999 record for that worksite. All the last year records for all the worksite were deleted, as change in VTR for them cannot be calculated. This associated data set now consisting of 25459 records (full sample data) was used to build the models described in this report.

Table 1: Los Angeles Data variable description and grouping

\begin{tabular}{|c|c|c|c|}
\hline No. & Field Name & Description & Grouping \\
\hline 1 & Motorcycle & Share of employees commuting by motorcycle & \\
\hline 2 & CAR1 & Share of employees commuting alone in car & \\
\hline 3 & CAR2 & Share of employees commuting two person carpool & \\
\hline 4 & CAR3 & Share of employees commuting three person carpool & \\
\hline 5 & CAR4 & Share of employees commuting four person carpool & \\
\hline 6 & CAR5 & Share of employees commuting five person carpool & \\
\hline 7 & CAR6 & Share of employees commuting six person carpool & \\
\hline 8 & VAN_CUTR & Share of employees commuting in van & \\
\hline 9 & BUS & Share of employees commuting in bus & \\
\hline 10 & TRANSIT & Share of employees commuting using transit & \\
\hline 11 & WALK & Share of employees commuting walking & \\
\hline 12 & BIKE & Share of employees commuting using bike & \\
\hline 13 & TELECOMMUTE & Share of employees telecommuting & \\
\hline 14 & CWW336 & Share of $3 / 36 \mathrm{CWW}$ days off & \\
\hline 15 & CWW440 & Share of $4 / 40 \mathrm{CWW}$ days off & \\
\hline 16 & CWW980 & Share of $9 / 80 \mathrm{CWW}$ days off & \\
\hline 17 & TARGET_VTR & Target VTR required (3 zones) & \\
\hline 18 & BFL & Passenger Loading Areas & FACILITY_AMENITIES \\
\hline 19 & BFO & Other Facility Improvements & FACILITY_AMENITIES \\
\hline 20 & BFP & Preferential Parking Areas & FACILITY_AMENITIES \\
\hline 21 & BFR & Bike Racks and Bike Lockers & FACILITY_AMENITIES \\
\hline 22 & BFS & Shower and Lockers & FACILITY_AMENITIES \\
\hline 23 & BGA & TMA/TMO Provided Guaranteed Return Trip & RIDE_HOME \\
\hline 24 & BGC & Company Vehicle Guaranteed Return Trip & RIDE_HOME \\
\hline 25 & BGE & Emergencies Guaranteed Return Trip & RIDE_HOME \\
\hline 26 & BGO & Other Guaranteed Return Trip Program & RIDE_HOME \\
\hline 27 & BGR & Rental Car Guaranteed Return Trip & RIDE_HOME \\
\hline 28 & BGT & Taxi Guaranteed Return Trip & RIDE_HOME \\
\hline 29 & BGU & Unscheduled Overtime Guaranteed Return & RIDE_HOME \\
\hline 30 & $\mathrm{BHF}$ & Flextime for Ride sharers (Work Shifts) & FLEXTIME \\
\hline 31 & BHG & Flextime for Ride sharers (Grace Period) & FLEXTIME \\
\hline 32 & BMC & Commuter Information Center & MARKETING \\
\hline 33 & BMF & Commuter Fairs (Marketing) & MARKETING \\
\hline 34 & BMG & Focus Groups (Marketing) & MARKETING \\
\hline 35 & BMM & Posted Materials (Marketing) & MARKETING \\
\hline 36 & BMN & New Hire Orientation (Marketing) & MARKETING \\
\hline 37 & BMO & Other Marketing Elements & MARKETING \\
\hline 38 & BMP & Personal Communication (Marketing) & MARKETING \\
\hline 39 & BMR & Company Recognition (Marketing) & MARKETING \\
\hline
\end{tabular}




\begin{tabular}{|c|c|c|c|}
\hline No. & Field Name & Description & Grouping \\
\hline 40 & BMS & Special Interest Club (Biking, Walking) & MARKETING \\
\hline 41 & BMT & TMA/TMO Membership (Marketing) & MARKETING \\
\hline 42 & BMW & Written Materials (Marketing) & MARKETING \\
\hline 43 & BMZ & ZipCode Meetings (Marketing) & MARKETING \\
\hline 44 & BRC & Regional Commuter Management Agency & RS_MATCH \\
\hline 45 & BRE & Employer-Based Rideshare Matching System & RS_MATCH \\
\hline 46 & DA & Transportation Allowances & FINANCIAL \\
\hline 47 & DFB & On-Going Bike-to-Work Subsidies & FINANCIAL \\
\hline 48 & DFC & On-Going Carpooling Subsidies & FINANCIAL \\
\hline 49 & DFI & Introductory Transit Passes or Subsidies & COMMTAX \\
\hline 50 & DFO & Other Direct Financial Subsidies & FINANCIAL \\
\hline 51 & DFS & Subsidized Vanpool Seats & COMMTAX \\
\hline 52 & DFT & On-Going Transit Subsidies & COMMTAX \\
\hline 53 & DFV & On-Going Vanpooling Subsidies & COMMTAX \\
\hline 54 & DFW & On-Going Walk-to-Work Subsidies & FINANCIAL \\
\hline 55 & DNA & Auto Services (Fuel, Oil, Tune-Up) & DIRECT_NONFINANCIAL \\
\hline 56 & DNC & Gift Certificates & DIRECT_NONFINANCIAL \\
\hline 57 & DNF & Free Meals & DIRECT_NONFINANCIAL \\
\hline 58 & DNO & Other Direct Non-Financial Incentives & DIRECT_NONFINANCIAL \\
\hline 59 & DNP & Catalogue Points & DIRECT_NONFINANCIAL \\
\hline 60 & DNT & Additional Time Off with Pay & DIRECT_NONFINANCIAL \\
\hline 61 & DPC & Increased Parking Costs for Drive Alones & PARKMGT\$ \\
\hline 62 & DPO & Other Parking Management Strategies & PARKMGT\$ \\
\hline 63 & DPS & Subsidized Parking for Ridesharers & PARKMGT\$ \\
\hline 64 & DTH & Work at Home (TeleCommuting) & TELE \\
\hline 65 & DTS & Work at Satellite Center (TeleCommuting) & TELE \\
\hline 66 & DW_3 & 3/36 Compressed Work Week Schedule & COMPRESSED \\
\hline 67 & DW_4 & 4/40 Compressed Work Week Schedule & COMPRESSED \\
\hline 68 & DW_9 & 9/80 Compressed Work Week Schedule & COMPRESSED \\
\hline 69 & DWO & Other Compressed Work Week Schedule & COMPRESSED \\
\hline 70 & IBO & Other Employee Benefits and Services & ONSITE \\
\hline 71 & IBP & Drawings, Free Meals, Certificates, etc. & DIRECT_NONFINANCIAL \\
\hline 72 & IBV & Company Owned/Leased Vanpools & VANPOOL \\
\hline 73 & ISC & On-Site Childcare Service & ONSITE \\
\hline 74 & ISO & Other On-Site Services & ONSITE \\
\hline 75 & ISS & Cafeteria, ATM's, Postal, Fitness Center & ONSITE \\
\hline 76 & IST & Transit Information or Pass Sales & ONSITE \\
\hline 77 & 000 & Other Not Classified by Other Codes & UNCLASSIFIED \\
\hline 78 & $\mathrm{XXX}$ & Incentives not Required & NOINCENTIVES \\
\hline 79 & FACILITY_AMENITIES & Grouped incentives & \\
\hline 80 & RIDE_HOME & Grouped incentives & \\
\hline 81 & FLEXTIME & Grouped incentives & \\
\hline 82 & MARKETING & Grouped incentives & \\
\hline 83 & RS_MATCH & Grouped incentives & \\
\hline 84 & FINANCIAL & Grouped incentives & \\
\hline 85 & PARKMGT\$ & Grouped incentives & \\
\hline 86 & TELE & Grouped incentives & \\
\hline 87 & COMPRESSED & Grouped incentives & \\
\hline 88 & VANPOOL & Grouped incentives & \\
\hline 89 & ONSITE & Grouped incentives & \\
\hline 90 & DIRECT_NONFINANCIAL & Grouped incentives & \\
\hline 91 & OTHER & Grouped incentives & \\
\hline 92 & COMMTAX & Grouped incentives & \\
\hline 93 & VTR_CUTR & Vehicle trip rate & \\
\hline 94 & EMP_TRIPS_CUTR & Total Employee trips & \\
\hline 95 & VEH_TRIPS_CUTR & Total Vehicle trips & \\
\hline 96 & Delta_VTR_CUTR & Change in vehicle trip rate between two years & \\
\hline
\end{tabular}




\section{TUCSON DATA}

The Tucson data was obtained from Pima County Association of Governments and consisted of 1,438 total records from 317 company worksites. Each Tucson record was similar to a Los Angeles data record in that it contained information related to worksite characteristics such as number of employees, shares of the different modes of transportation used by employees for commuting; the different incentives offered etc. Each record included a total of 58 fields, which are shown in Table 2. Using the information about the incentive groupings for Los Angeles data, several Tucson incentives were combined together in similar compatible Los Angeles grouped incentives. The data collected was from year 1996 to 2001. Some worksites collected data only for a subset of these years. The vehicle trip rate (VTR) for each worksite was calculated from the given data using the formula -

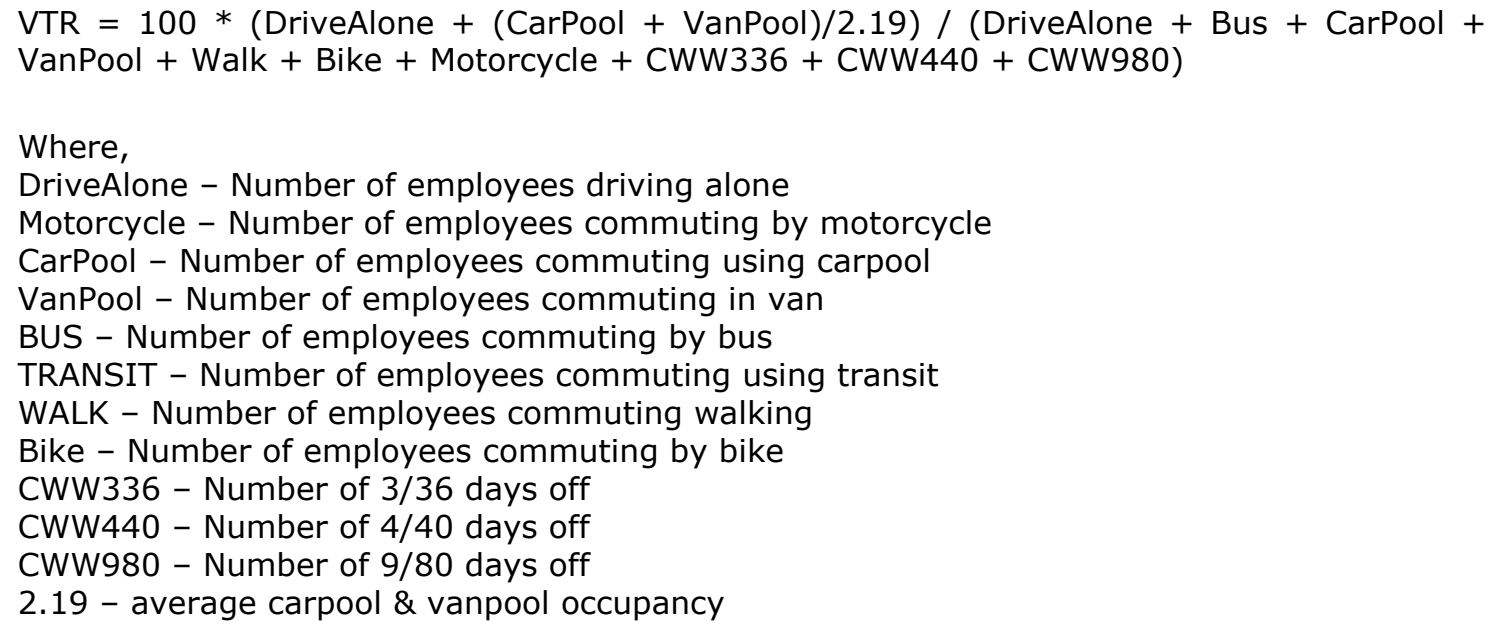

The change in VTR (Delta_VTR) was calculated similar to the way it was calculated for Los Angeles data and the last year records were removed. 
Table 2: Tucson Data variable description and grouping

\begin{tabular}{|c|c|c|c|}
\hline No. & Field & Description & Grouping \\
\hline 1 & \begin{tabular}{|l|} 
PermId \\
\end{tabular} & Id number of the employer & \\
\hline 2 & PlanYr & Plan year & \\
\hline 3 & AloneShare & Alone share & \\
\hline 4 & BusShare & Bus share & \\
\hline 5 & CVpoolShare & Carpool + Vanpool share & \\
\hline 6 & WalkShare & walk share & \\
\hline 7 & McycleShare & Motorcycle share & \\
\hline 8 & BcycleShare & Bicycle share & \\
\hline 9 & HiMiles & Average Miles traveled & \\
\hline 10 & HiMinute & Average minutes traveled & \\
\hline 11 & CWW336 & 3/36 compressed work week share & \\
\hline 12 & CWW440 & 4/40 compressed work week share & \\
\hline 13 & CWW980 & $8 / 80$ compressed work week share & \\
\hline 14 & VTR & Vehicle trip rate (in fraction) & \\
\hline 15 & NoEmp & Number of employees & \\
\hline 16 & $\mathrm{AC}$ & Alternate Mode Information & MRKT \\
\hline 17 & $\mathrm{AQ}$ & Post Air Quality Information & MRKT \\
\hline 18 & AW & Adjusted Work Hours & FLEX \\
\hline 19 & $\mathrm{BP}$ & Bus Pass Sales on Site & ONSITECONV \\
\hline 20 & BR & Bicycle Racks & FACILITY_AMENITIES \\
\hline 21 & BS & Bus Subsidy & COMMTAX \\
\hline 22 & BV & Busing Vehicle & VANPOOL \\
\hline 23 & $\mathrm{CC}$ & Matching Service & RSMATCH \\
\hline 24 & $C D$ & Covered Parking & FACILITY_AMENITIES \\
\hline 25 & CG & Alternative Fuel Vehicles & VANPOOL \\
\hline 26 & $\mathrm{CP}$ & Carpool Subsidy & FINANCIAL \\
\hline 27 & $\mathrm{CS}$ & Coordination with Transit Provider & MRKT \\
\hline 28 & $\mathrm{CV}$ & Carpooling Vehicle & VANPOOL \\
\hline 29 & $\mathrm{CW}$ & Compressed Work Week & CWW \\
\hline 30 & DC & Daycare Facilities on Site & ONSITECONV \\
\hline 31 & $\mathrm{DQ}$ & Dissemination of Air Quality Information & MRKT \\
\hline 32 & DW & Drawing for Prizes & MRKT \\
\hline 33 & ES & Employee Shifts Between Sites & TELE \\
\hline 34 & EV & Alternate Mode Campaign & MRKT \\
\hline 35 & FP & Fee for Parking & PARKMGT\$ \\
\hline 36 & FW & Field Worker & UNCLASSIFIED \\
\hline 37 & GP & Guaranteed Ride Home Program & GRH \\
\hline 38 & IC & Information Center & MRKT \\
\hline 39 & IN & Incentives for Employees to Live Close & FINANCIAL \\
\hline 40 & IP & Incentive Programs & DIRECT_NONFIN \\
\hline 41 & MP & Map Board & RSMATCH \\
\hline 42 & NE & New Employee Information & MRKT \\
\hline 43 & NL & Newsletter Articles & MRKT \\
\hline 44 & PP & Preferred Parking & FACILITY_AMENITIES \\
\hline 45 & $\mathrm{RC}$ & Rideshare Committee & MRKT \\
\hline 46 & RP & Rebate not to Use Parking & PARKMGT\$ \\
\hline 47 & SA & Staging Area & FACILITY_AMENITIES \\
\hline 48 & SC & Carpool Campaign & MRKT \\
\hline 49 & SK & Speakers & MRKT \\
\hline 50 & SV & Shuttle Vehicle & VANPOOL \\
\hline 51 & SW & Showers/Lockers & FACILITY_AMENITIES \\
\hline 52 & TF & Transportation Fair & MRKT \\
\hline 53 & VC & Vanpooling Vehicle & MRKT \\
\hline 54 & VP & Vanpool Subsidy & COMMTAX \\
\hline 55 & VV & Vanpooling Vehicle & VANPOOL \\
\hline 56 & WB & Walking Campaign & MRKT \\
\hline 57 & $\mathrm{WH}$ & Work at Home & TELE \\
\hline 58 & WS & Bicycle Campaign & MRKT \\
\hline 59 & Delta_VTR & Change in VTR & Change in VTR \\
\hline
\end{tabular}




\section{WASHI NGTON DATA}

The Washington data was obtained from Washington State Department of Transportation and consisted of 2,482 total records from 1,038 company worksites. Each record represents information from a specific company worksite for a specific year. The information in a record includes worksite characteristics such as number of employees, the type of the company, shares of the different modes of transportation used by employees for commuting, the different incentives, and the preferences of the employees for the incentives they feel important. Each record included a total of 106 fields, which are shown in Table 3. The table also shows the grouping of the similar incentives as done for other datasets. For Washington, bi-yearly data were collected from 1995 to 2001. The vehicle trip rate for each worksite was calculated from the given data using the formula

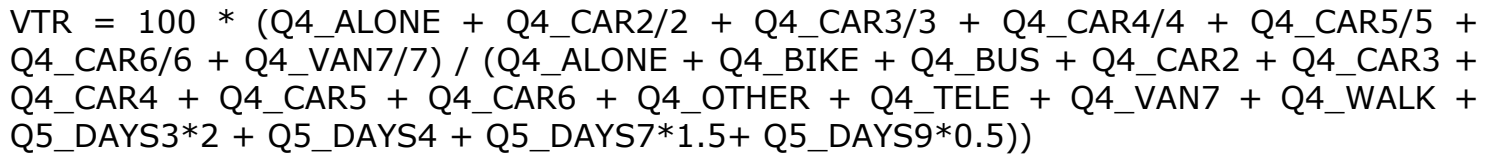

Where,

Q4_ALONE - Number of employees driving alone

Q4_CAR2- Number of employees commuting two together

Q4_CAR3 - Number of employees commuting three together

Q4_CAR4 - Number of employees commuting four together

Q4_CAR5 - Number of employees commuting five together

Q4_CAR6 - Number of employees commuting six together

Q4_VAN7 - Number of employees commuting in van

Q4_BUS - Number of employees commuting by bus

Q4_WALK - Number of employees commuting walking

Q4_BIKE - Number of employees commuting by bike

Q4_TELE - Number of employees telecommuting

Q5_DAYS3 - Number of employees on 3/36

Q5_DAYS4 - Number of employees on $4 / 40$

Q5_DAYS7 - Number of employees on $9 / 80$

Q5_DAYS9 - Number of employees on $9 / 80$

The change in VTR (Delta_VTR) was calculated similar to the way it was calculated for Los Angeles data and the last year records were removed. 
Table 3: Washington Data variable description and grouping

\begin{tabular}{|c|c|c|c|}
\hline No. & Field Name & Description & Grouping \\
\hline 1 & CTRID & CTRID & \\
\hline 2 & AnnReYR & Plan Year & \\
\hline 3 & NonProf & Non-profit organization & \\
\hline 4 & Agri & Agriculture organization & \\
\hline 5 & Finance & Finance organization & \\
\hline 6 & InfoServ & Info services organization & \\
\hline 8 & Retail & Retail organization & \\
\hline 9 & Manufact & Manufacturing organization & \\
\hline 10 & Services & Services organization & \\
\hline 11 & pubUtil & Public utilities organization & \\
\hline 12 & Construc & Construction organization & \\
\hline 17 & Union & Union & \\
\hline 18 & Shifts & Shifts & \\
\hline 19 & OnParkSp & Onsite Parking Spaces & \\
\hline 20 & OfParkSp & Offsite Parking Spaces & \\
\hline 21 & LOnParkP & Leased Onsite Parking Price & \\
\hline 22 & LOfParkP & Leased Offsite Parking Price & \\
\hline 23 & OwnOnPrk & Own Onsite Parking & \\
\hline 24 & OnPrkChr & Onsite Parking Charge & \\
\hline 25 & OwnOfPrk & Own Offsite Parking & \\
\hline 26 & OfPrkChr & Offsite Parking Charge & \\
\hline 33 & PstMatrl & Post Materials & \\
\hline 34 & Orientat & CTR Orientation & \\
\hline 35 & CTREvent & CTR Events & MRKT \\
\hline 36 & CTREmail & CTR E-mail & MRKT \\
\hline 37 & Articles & Articles & MRKT \\
\hline 38 & RideMApp & Ride match Apps & RS_MATCH \\
\hline 39 & WPycheck & With Paychecks & MRKT \\
\hline 40 & Drawings & Drawings & DIRECT_NONFINANCIAL \\
\hline 41 & Leave & Leave & DIRECT_NONFINANCIAL \\
\hline 42 & OthPromo & Other Promo & MRKT \\
\hline 43 & CovBikeN & Covered Bike Number & FACILITIES_AMENITIES \\
\hline 44 & UncovBkN & Uncovered Bike Number & FACILITIES_AMENITIES \\
\hline 45 & LockersN & Lockers Number & FACILITIES_AMENITIES \\
\hline 46 & ShowersN & Showers Number & FACILITIES_AMENITIES \\
\hline 47 & ShelterN & Shelters Number & FACILITIES_AMENITIES \\
\hline 48 & OthrAmeN & Other Amenities 1 Number & FACILITIES_AMENITIES \\
\hline 49 & CP-SpacN & Carpool Spaces Number & FACILITIES_AMENITIES \\
\hline 50 & VP-SpacN & Vanpool Spaces Number & FACILITIES_AMENITIES \\
\hline 51 & SOVPkch & SOV Parking Charge & PARKMGT \\
\hline 52 & SOVPkChN & SOV Parking Charge Number & PARKMGT \\
\hline
\end{tabular}




\begin{tabular}{|c|c|c|c|}
\hline No. & Field Name & Description & Grouping \\
\hline 63 & EmpPubM & Employees public match & RS_MATCH \\
\hline 64 & FVwrkEmp & FV work employees & \\
\hline 65 & EmpNo & Number of Employees & \\
\hline 66 & CWW3 & Percentages of employees on $3 / 36 \mathrm{CWW}$ & CWW \\
\hline 67 & CWW4 & Percentages of employees on $4 / 40 \mathrm{CWW}$ & CWW \\
\hline 68 & CWW5 & Percentages of employees on $5 / 40$ & \\
\hline 69 & CWW7 & Percentages of employees on $7 / 40 \mathrm{CWW}$ & CWW \\
\hline 70 & CWW9 & Percentages of employees on $9 / 80 \mathrm{CWW}$ & CWW \\
\hline 71 & CWWOTHER & Percentages of employees on other CWW & CWW \\
\hline 72 & AloneShr & Alone share & \\
\hline 73 & BikeShr & Bike share & \\
\hline 74 & BusShr & Bus share & \\
\hline 75 & CarShr & Cars hare & \\
\hline 76 & OtherShr & Other share & \\
\hline 77 & TeleShr & Tele share & \\
\hline 78 & VanShr & Van share & \\
\hline 79 & WalkShr & Walk share & \\
\hline 80 & DSVTC2wk & Days saved telecommuting in two weeks & \\
\hline 81 & AdminSh & Administration job Share & \\
\hline 82 & CrPrLaSh & Craft/Production/Labor Share & \\
\hline 83 & MgntSh & Management job Share & \\
\hline 84 & SaleMktSh & Sales/Marketing job Share & \\
\hline 85 & CstSrvSh & Customer Service job Share & \\
\hline 86 & OtherSh & Other job Share & \\
\hline 87 & ProTecSh & Professional/Technical job Share & \\
\hline 88 & PEmpPCar & Prefer provide car for work & \\
\hline 89 & PTrlunch & Employee Prefer Transport during lunch & \\
\hline 90 & PreGRH & Employee Prefer GRH & \\
\hline 91 & PflxCVpB & Employee Prefer flex to meet CVpool bus & \\
\hline 92 & PFinance & Employee Prefer financial incentive & \\
\hline 93 & PDsCVpSp & Employee Prefer reserved discounted CVpool space & \\
\hline 94 & PPrIHCVp & Employee Prefer Personalized help for CVpool & \\
\hline 95 & PCovbkPk & Employee Prefer covered bicycle parking & \\
\hline 96 & PLckAShw & Employee Prefer lockers \& showers & \\
\hline 97 & POnChild & Employee Prefer onsite childcare & \\
\hline 98 & PreCWW & Employee Prefer CWW & \\
\hline 99 & PreTele & Employee Prefer to telecommute & \\
\hline 100 & Pimptran & Employee Prefer improved access to transit & \\
\hline 101 & SOV & SOV & \\
\hline 102 & VMT & VMT & \\
\hline 103 & VTR & VTR & \\
\hline 104 & CBD & Central Business District & \\
\hline 105 & suburb & Suburban area & \\
\hline 106 & outside & Outside suburban area & \\
\hline 107 & DeltaVTR & DeltaVTR & \\
\hline
\end{tabular}




\section{I I. MODEl BUI LDI NG APPROACHeS}

The above cleaned data and its subsets were used in the model building process. Two different approaches were used in model building: non-linear neural networks and linear statistical regression models. Regression models identify the simple linear relationships between the independent inputs and the dependent output variable, whereas neural network models can explain much more complex non-linear relationships between the independent inputs and dependent variables. Therefore, the results provided by the regression models can be used as the baseline for the comparison of the various neural network models.

\section{NeURAL NetWORKS}

Neural networks are essentially a group of highly interconnected and relatively simple computational units, as illustrated in Figure 1. Each of these computational units performs processing of its inputs to produce a single output. The neural network connects the output of each unit to the inputs of many other units through different weights.

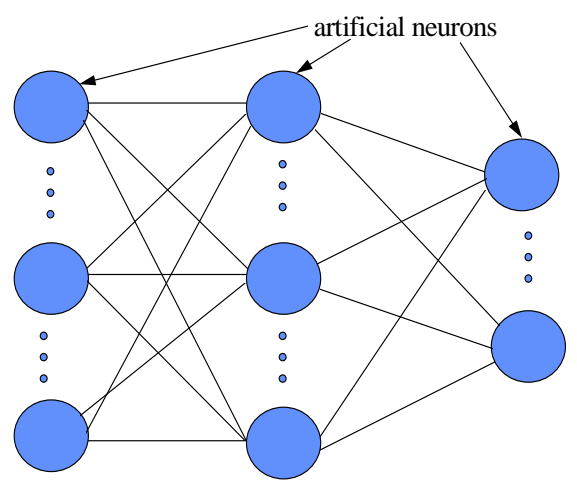

Figure 1: Neural network consisting on interconnected neuron

Figure 2 shows a typical artificial neuron (i.e., any single individual neuron) which adds all of its weighted inputs and uses a sigmoid output function to generate its output. Other output functions, such as the hyperbolic tangent, are sometimes used. As can be seen in Figure 2, neural network learning consists of finding the correct weighting of the input data so that the model of best fit may be found. 


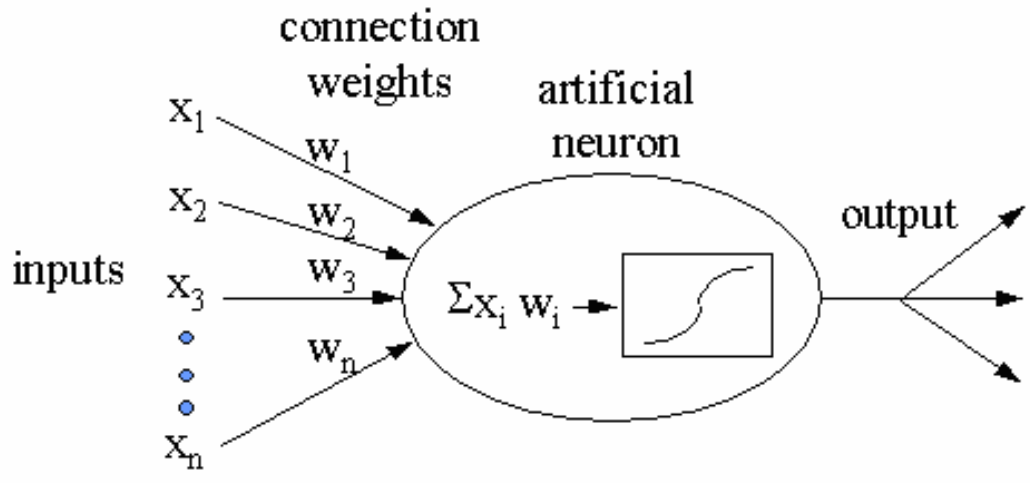

Figure 2: Artificial neuron with inputs and outputs

To help understand the process followed to build the model, defining several key terms is necessary.

Training is a process that uses one of several learning techniques to modify the weights in an orderly fashion. The training set of data is a list of paired input and desired output patterns used in supervised training. All of the information the network needs to learn must be in the training set. The inputs can be numbers, symbols, or pictures.

The testing set is an extract of the training set used while building the model to prevent over-fitting. Over-fitting the training data can occur when the neural network produces a nonlinear model that fits the training data perfectly, but fits the test data very poorly. The goal is to fit both the training and testing data with approximately the same overall error. Therefore, the testing data set is used to analyze the model's ability to interpolate the training/testing data regularly during training. Training is halted when the test performance starts to degrade.

The validation set is independent of the training/testing set and typifies the data that will be seen by the model in the outside world.

Figure 3 illustrates the type of network that is most popular today - a multi-layer, fully-connected, feed forward neural network. These types of networks consist of two or more layers of individual neurons. Each neuron in a given layer receives inputs from all the neurons in the previous layer. The output of the previous layer is input to all neurons in the succeeding layer. The middle layers, between the input and output layers are called hidden layers since they are not directly accessible. The sigmoid or the hyperbolic tangent allows each computational unit to implement a nonlinear mapping between its inputs and output. This allows the networks to model nonlinear relationships that may exist in the data. Once this network is 
trained, it will produce the desired m-dimensional output given an $\mathrm{n}$ dimensional input. The records provide the desired input-output associations used to train the network.

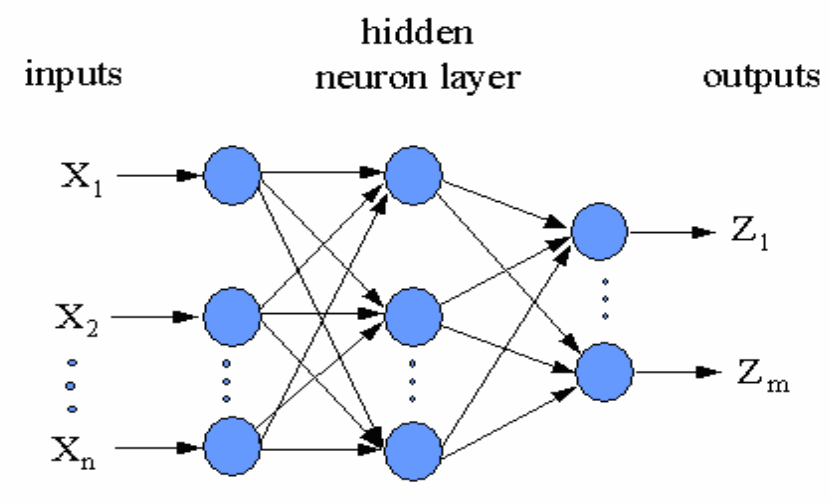

Figure 3: Typical multi-layered fully connected feed forward neural network

The popularity of these types of networks is mostly due to two factors. First, a two-layer feed forward network is capable of implementing any association between inputs and outputs. The second factor that accounts for the popularity of these types of networks is the existence of a welldefined training method called back propagation. This training method can find the weight values that will allow the neural network to produce the desired $\mathrm{m}$-dimensional output given an $\mathrm{n}$-dimensional input.

Before training a network using the back propagation method, the network builder must identify inputs and outputs. This is a critical step for building an accurate neural network and should be done by someone aware of the application domain. During training, that portion of a record identified as input is presented to the network. If the output of the network differs from the output portion of the record, then the neural network changes the weights of the network. The back propagation method specifies what changes to make to the weights so that the neural network reduces the difference between the actual and the desired network output. All of the training records are presented to the network, and corrections made. This makes up one training cycle. Typically, training a network requires many training cycles until the cumulative errors of all records in one cycle are below an acceptable level. Thus back propagation uses the records in the training data repeatedly to change the weights in the network so that the difference between the desired and the actual network output is below an acceptable level. 


\section{General Network Structure}

For the reasons mentioned above, the type of neural network selected to predict the change in VTR was a multi-layer, fully-connected, feed forward type. Neural network model builders have applied these types of networks successfully for prediction and classification problems in a variety of fields.

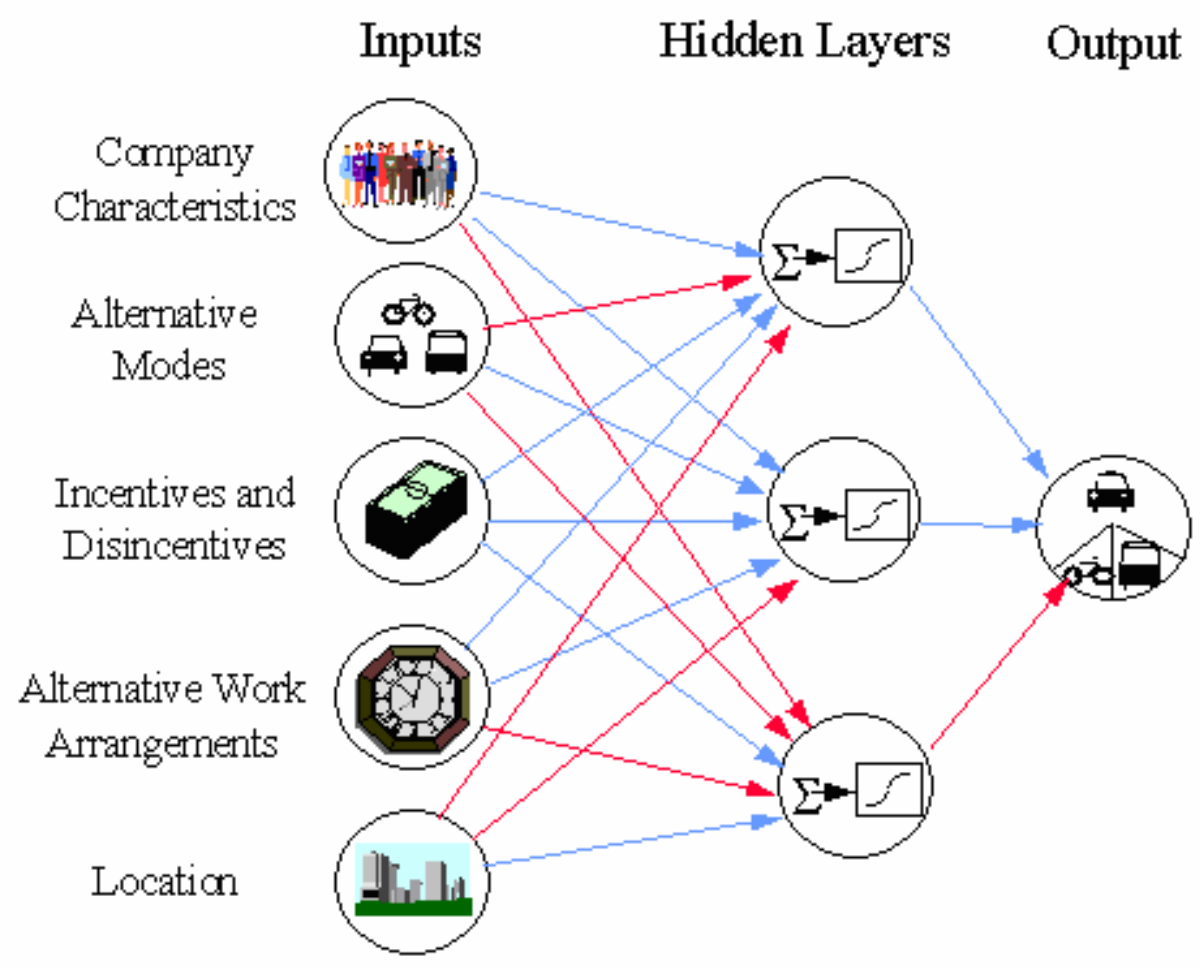

Figure 4: Network structure for Worksite trip reduction model

Figure 4 shows an overview of the network's structure. All of the inputs to the network and the output have been described in this report. The goal of this network is to predict the change in VTR that a company will obtain due to the specific combination of incentive plans selected by the company and the specific characteristics of that company. The neural network determines the number of hidden nodes and layers during training and will be different from those illustrated.

\section{Software Used to Build the Neural Networks}

Neuralware's NeuralWorks Predict v5.0, a plug-in in Microsoft's Excel was used for building the neural network models. Microsoft's database program, Access, was also used to manipulate the data before training the network. The hardware used was a $1 \mathrm{GHz}$ Pentium III PC with 512 Mbytes of RAM.

Predict simplifies the different aspects of the neural network training process by allowing the network builder to select many parameters that can 
affect the performance of the final model. Researchers were able to customize the following key parameters:

1. The types and maximum number of computational units to use.

2. The type of error evaluation function. For example, one error evaluation function may emphasize higher correlation between predicted and desired network output, while another may emphasize greater accuracy of the predicted quantity.

3. Whether to consider noise (i.e., errors in the data caused by individual differences between companies and people) in the training data.

4. In what proportion to break up the data used for training the network and the data used to test the trained network.

5. Whether to eliminate as input to the network those input variables that had little correlation with the output variable.

\section{REGRESSION ANALYSIS}

Regression models were built using the SPSS system, release 11.1 for Windows.

To provide a baseline of comparison of the ability of the neural network model to predict changes in VTR correctly, an independent model was first created by means of factor analysis and stepwise regression.

Initial regressions suffered from multi-collinearity within the data. Since many independent variables were inter-correlated, a possibility exists that the coefficients resulting from model runs would not fully reflect the effects of each of the independent variables. The initial approach to eliminating the effects of the multi-collinearity was to run a factor analysis.

Generally, factor analysis is used as a data reduction technique. The analytical procedure involves creating uncorrelated (orthogonal) combinations of the initial dependent variables.

In common practice, the purpose of the analysis is to reduce a mass of variables to a reasonable number of elements (e.g., 10), which the analyst can understand and explain. Often, the selection of factors to use is limited to those that explain at least as much variance as an independent variable, i.e., the output factor has an eigenvalue of at least one.

However, here, the purpose was to create a series of uncorrelated factors to use as independent variables in regression analysis, without attempting to explain the meaning of the factors. When factor analysis is used in hard science applications, factors are commonly retained to the point where 95 percent of the variance in the data is explained. Some factor analysts even maintain that any factor with a positive eigenvalue is relevant for 
analysis (Hair, 1984). Since a stepwise regression procedure was to be used later for model building, CUTR researchers decided that the stepwise procedure would determine the significance of the factors produced by the analysis, rather than limiting the factors output through analysis of eigenvalues.

The stepwise regression was set to accept variables that significantly improved the model at a 95 percent confidence level. When the analysis had been completed, the factors were then reconverted into the original component independent variables. The conversion was made by multiplying the coefficients assigned by the regression model to the factors by the matrix of the factor loadings of the original variables. The resulting equations predicted the change in VTR.

No reduction in the number of predictor variables was obtained by using this approach. In fact, compared to stepwise regressions, the number of predictor variables was larger because nearly every input variable loaded onto a factor at some point. Even reducing the factors used to those with eigenvalues above 1.0 would not have helped this situation.

The second step taken to resolve this difficulty was to remove correlated variables. While this step risked reducing the predictive power of the model, the resulting coefficients would be much more easily interpretable. Also, a stepwise selection of variables reduces the data required to make a prediction. This should eventually reduce the burden placed on model users.

The actual finding was that taking this step only marginally diminished the explanatory power of the model. The overall predictive accuracy of the results, as shown in later sections, was only slightly reduced no matter the method of regression model building.

In order to understand the results listed in later chapters, one must understand the methods used in SPSS regression. The stepwise methodology has been briefly explained previously, but the forward, backward, forced enter, and stepwise will be discussed further so that the reader will understand the processes involved in the varying methodologies of model building via regression.

Forward regression is very much like stepwise regression in that SPSS will first compute which predictor variable has the largest bivariate correlation with the dependent variable. From this point, the SPSS program will then add in various other variables which explain that largest amount of variance and these additional variables will only be included if they explain a significant amount of additional variance. The forward regression process stops when there are no more variables remaining that can explain a significant amount of the variance. 
In the case of backward regression, the program enters all of the independent variables and then removes them one by one, based upon a preset significance value. The default value in this case removes all variables which don't explain at least 90 percent of the variance. The backward regression process stops when there are no more variables remaining for removal that meet this requirement.

Stepwise regression combines both forward and backward methodologies. It is a complex procedure in that it will remove or add variables as the inter-correlations change between variables already in the regression formula. That is, a variable which significantly explained the variance in the dependent variable is weakened by the addition of another variable and is therefore removed by the program. Stepwise is the most popular of all regression methods.

Forced enter regression method is more simple than stepwise, forward, or backward, in that the program simply accepts all of the specified variables and builds a regression equation regardless of the significance levels of the entered independent variables.

\section{Measuri ng the Performance of Trai ned Models}

As stated earlier, the dataset selected for building a neural network/regression models is divided in two disjoint sets "training/testing set" and "validation set". In case of neural network models, the training/testing set is once more split into two separate sets, the "training set" and the "testing set". The training set is used to train the network while the testing set is used to test the performance of the network as it is being trained and control the number of hidden units in the network. By default, Predict uses 70 percent of the random data from training/testing set as the training set and remaining 30 percent as the test set, although the network builder can change these values to any other proportions. The whole training/testing set is used for training by the statistical software for building the regression models. The validation set which is a representative sample of the original input data is used in the final performance evaluation of the models. The neural network/statistical software does not use the validation set in building the model.

To get some raw estimates of the accuracies of the predicted Delta_VTR (change in VTR), the range of the continuous variable Delta_VTR is discretized into 8 bin/classes so that each bin contains approximately $1 / 8^{\text {th }}$ of the data. Since most of the changes in VTR are small from one period to the next, the bin classification approach to assessing the model performance helps focus on plans with large changes in VTR. Also since each bin has to contain approximately same amount of data, the bin ranges used for the three datasets are not consistent. Two accuracies, the "Exact Accuracy" and the "One-off Accuracy" measures are used. 
The "Exact Accuracy" measures, for how many records the predicted bin was exactly same as actual bin. The "One-off Accuracy" measures, for how many records the predicted bin was same as actual bin or one of its neighboring bins.

In the next section, we do the comparison of the various neural network and regression models using these three performance measures

The first performance measure is the 'Bin Classification Accuracy on moderate range of change in VTR (i.e. bins a2 to a5)'. The second performance measure is the 'Bin Classification Accuracy on full range of change in VTR (i.e. all bins)' and the third measure is the R-square between the actual Delta_VTR and the Predicted Delta_VTR. R-square is a statistical term for the amount of variance for which the regression formula is able to account.

The formula used for calculating R-square is

Where,

$$
r=\frac{\sum(x-\bar{x})(y-\bar{y})}{\sqrt{\sum(x-\bar{x})^{2} \sum(y-\bar{y})^{2}}}
$$

$\mathrm{x}=$ Actual Delta_VTR

$\mathrm{y}=$ Predicted Delta_VTR

It has to be kept in mind that the first two performance measures, which assess the accuracy over a distribution, may be more useful than the $\mathrm{R}$-square variance measure. It was found that often the models had a poor R-square but better Bin Classification Accuracies on moderate and full range of change in VTR.

\section{DATASETS MANI PULATI ONS}

Various data manipulations were performed on the different datasets to improve the performance of the models built on them. The individual incentives in the datasets were replaced by the grouped incentives, thus reducing the total number of variables in the dataset. This helped in reducing the complexity and adding more explanatory power to of the models. In some cases the data in bins lying in moderate range of change in VTR was over-sampled, meaning that the samples in those bins were duplicated to give more importance to these samples as against to the data in other ranges of change in VTR was under-sampled, meaning that some samples in these bins were dropped to reduce their importance. Models were also built on the data which was manipulated by both grouping of incentives and over/under sampling of data in some bins. 


\title{
IV. MODEL BUI LDING
}

\author{
LOS ANGELes MODELS
}

\section{Phase I: Los Angeles Full Sample Data}

The Los Angeles dataset consisted of 25,459 total examples. For datasets from other areas, ten percent of the dataset was left aside as the validation set while the rest of the data became the training/testing set. However, because of the large size of the Los Angeles dataset, twenty percent of the data was left aside as the validation set. The ranges of the eight bins based on the change in VTR values and the number of examples in each bin for validation and training/testing set are shown in Table 4.

Table 4: Los Angeles Full Sample Data - No. of Records in bins

\begin{tabular}{|c|c|c|c|c|c|c|c|c|c|}
\hline \multirow{2}{*}{ range } & \multirow{2}{*}{ \# of records } & \multicolumn{6}{|c|}{ Bin ranges over Change in Vehicle Trip Rate (Delta_VTR) } \\
\cline { 3 - 9 } & & $\mathbf{> - 7}$ & [- 7 to - 4) & [- - 4 to - 2) & [- 2 to -0) & [-0 to 1) & [1 to 2.5) & [2.5 to 5) & 5 >= \\
\hline Bin Number & & $\mathbf{a 1}$ & $\mathbf{a 2}$ & $\mathbf{a 3}$ & $\mathbf{a 4}$ & $\mathbf{a 5}$ & $\mathbf{a 6}$ & $\mathbf{a 7}$ & $\mathbf{a 8}$ \\
Validation & $\mathbf{2 5 3 7}$ & 347 & 286 & 356 & 423 & 237 & 264 & 262 & 362 \\
Training & $\mathbf{2 2 9 2 2}$ & 3087 & 2969 & 2943 & 3988 & 2155 & 2260 & 2612 & 2908 \\
\hline
\end{tabular}

A first attempt to build neural network models using all the variables present in the data resulted in very poor performance. So, different linear regression modeling approaches were used to select different sets of variables. Forced Enter, Stepwise, Forward and Backward regression approaches yielded different subsets of variables as indicated in the table below. Forced Enter consists of entering all variables to build the model, regardless of significance in explaining variance. Forward method involves the entry of variables one at a time, based on the significance of each variable. Backward method involves entering all variables and removing each one by one, according to a preset significance value. Finally, Stepwise method utilizes both forward and backward methods, obtaining the best set of variables for the regression equations.

After the different methodologies were applied to building the SPSS regression models, the neural network models were built using each of the different set of variables obtained from each of the four regression building methods, forced enter, stepwise, forward, and backward. Table 5 shows the different variables selected by each of the different SPSS regression methods. These variables were then used to train the neural net models. 
Table 5: Variables selected by different regression methods

\begin{tabular}{|c|c|c|c|c|}
\hline \multirow{2}{*}{ Names } & \multicolumn{4}{|c|}{ Variables selected using regression method } \\
\hline & Force Enter & stepwise & Forward & Backward \\
\hline Share employees commuting by motorcycle & $\sqrt{ }$ & $\sqrt{ }$ & $\sqrt{ }$ & \\
\hline Share employees commuting alone in car & $\sqrt{ }$ & $\sqrt{ }$ & $\sqrt{ }$ & \\
\hline Share employees commuting two together & $\sqrt{ }$ & $\sqrt{ }$ & $\sqrt{ }$ & $\sqrt{ }$ \\
\hline Share employees commuting three together & $\sqrt{ }$ & $\sqrt{ }$ & $\sqrt{ }$ & $\sqrt{ }$ \\
\hline Share employees commuting four together & $\sqrt{ }$ & & & $\sqrt{ }$ \\
\hline Share employees commuting five together & $\sqrt{ }$ & $\sqrt{ }$ & $\sqrt{ }$ & \\
\hline Share employees commuting six together & $\sqrt{ }$ & & & \\
\hline Share employees commuting in van & $\sqrt{ }$ & & & $\sqrt{ }$ \\
\hline Share employees commuting in bus & $\sqrt{ }$ & & & $\sqrt{ }$ \\
\hline Share employees commuting using transit & $\sqrt{ }$ & & & $\sqrt{ }$ \\
\hline Share employees commuting walking & $\sqrt{ }$ & & & $\sqrt{ }$ \\
\hline Share employees commuting using bike & $\sqrt{ }$ & $\sqrt{ }$ & $\sqrt{ }$ & $\sqrt{ }$ \\
\hline Share employees tele-commuting & $\sqrt{ }$ & $\sqrt{ }$ & $\sqrt{ }$ & $\sqrt{ }$ \\
\hline Share of $3 / 36$ CWW days off & $\sqrt{ }$ & $\sqrt{ }$ & $\sqrt{ }$ & $\sqrt{ }$ \\
\hline Share of $4 / 40 \mathrm{CWW}$ days off & $\sqrt{ }$ & $\sqrt{ }$ & $\sqrt{ }$ & $\sqrt{ }$ \\
\hline Share of $9 / 80$ CWW days off & $\sqrt{ }$ & $\sqrt{ }$ & $\sqrt{ }$ & $\sqrt{ }$ \\
\hline Target VTR required ( 3 zones) & $\sqrt{ }$ & $\sqrt{ }$ & $\sqrt{ }$ & $\sqrt{ }$ \\
\hline Passenger Loading Areas & $\sqrt{ }$ & & & \\
\hline Other Facility Improvements & $\sqrt{ }$ & $\sqrt{ }$ & $\sqrt{ }$ & $\sqrt{ }$ \\
\hline Preferential Parking Areas & $\sqrt{ }$ & & & $\sqrt{ }$ \\
\hline Bike Racks and Bike Lockers & $\sqrt{ }$ & & & $\sqrt{ }$ \\
\hline Shower and Lockers & $\sqrt{ }$ & & & \\
\hline TMA/TMO Provided Guaranteed Return Trip & $\sqrt{ }$ & & & \\
\hline Company Vehicle Guaranteed Return Trip & $\sqrt{ }$ & $\sqrt{ }$ & $\sqrt{ }$ & $\sqrt{ }$ \\
\hline Emergencies Guaranteed Return Trip & $\sqrt{ }$ & $\sqrt{ }$ & $\sqrt{ }$ & $\sqrt{ }$ \\
\hline Other Guaranteed Return Trip Program & $\sqrt{ }$ & $\sqrt{ }$ & $\sqrt{ }$ & $\sqrt{ }$ \\
\hline Rental Car Guaranteed Return Trip & $\sqrt{ }$ & & & \\
\hline Taxi Guaranteed Return Trip & $\sqrt{ }$ & $\sqrt{ }$ & $\sqrt{ }$ & $\sqrt{ }$ \\
\hline Unscheduled Overtime Guaranteed Return & $\sqrt{ }$ & & & $\sqrt{ }$ \\
\hline Flextime for Ridesharers (Work Shifts) & $\sqrt{ }$ & & & \\
\hline Flextime for Ridesharers (Grace Period) & $\sqrt{ }$ & & & \\
\hline Commuter Information Center & $\sqrt{ }$ & & & \\
\hline Commuter Fairs (Marketing) & $\sqrt{ }$ & & & \\
\hline Focus Groups (Marketing) & $\sqrt{ }$ & & & \\
\hline Posted Materials (Marketing) & $\sqrt{ }$ & $\sqrt{ }$ & $\sqrt{ }$ & $\sqrt{ }$ \\
\hline New Hire Orientation (Marketing) & $\sqrt{ }$ & $\sqrt{ }$ & $\sqrt{ }$ & $\sqrt{ }$ \\
\hline Other Marketing Elements & $\sqrt{ }$ & $\sqrt{ }$ & $\sqrt{ }$ & $\sqrt{ }$ \\
\hline Personal Communication (Marketing) & $\sqrt{ }$ & & & \\
\hline Company Recognition (Marketing) & $\sqrt{ }$ & & & $\sqrt{ }$ \\
\hline Special Interest Club (Biking, Walking) & $\sqrt{ }$ & $\sqrt{ }$ & $\sqrt{ }$ & $\sqrt{ }$ \\
\hline TMA/TMO Membership (Marketing) & $\sqrt{ }$ & & & \\
\hline Written Materials (Marketing) & $\sqrt{ }$ & & & $\sqrt{ }$ \\
\hline ZipCode Meetings (Marketing) & $\sqrt{ }$ & & & \\
\hline Regional Commuter Management Agency & $\sqrt{ }$ & & & $\sqrt{ }$ \\
\hline Employer-Based Rideshare Matching System & $\sqrt{ }$ & & & $\sqrt{ }$ \\
\hline Transportation Allowances & $\sqrt{ }$ & & & \\
\hline On-Going Bike-to-Work Subsidies & $\sqrt{ }$ & & & \\
\hline On-Going Carpooling Subsidies & $\sqrt{ }$ & $\sqrt{ }$ & $\sqrt{ }$ & $\sqrt{ }$ \\
\hline Introductory Transit Passes or Subsidies & $\sqrt{ }$ & & & \\
\hline Other Direct Financial Subsidies & $\sqrt{ }$ & & & \\
\hline Subsidized Vanpool Seats & $\sqrt{ }$ & & & $\sqrt{ }$ \\
\hline On-Going Transit Subsidies & $\sqrt{ }$ & & & \\
\hline On-Going Vanpooling Subsidies & $\sqrt{ }$ & & & $\sqrt{ }$ \\
\hline On-Going Walk-to-Work Subsidies & $\sqrt{ }$ & & & $\sqrt{ }$ \\
\hline Auto Services (Fuel, Oil, Tune-Up) & $\sqrt{ }$ & & & \\
\hline Gift Certificates & $\sqrt{ }$ & & & \\
\hline Free Meals & $\sqrt{ }$ & & & $\sqrt{ }$ \\
\hline Other Direct Non-Financial Incentives & $\sqrt{ }$ & & & \\
\hline Catalogue Points & $\sqrt{ }$ & & & \\
\hline Additional Time Off with Pay & $\sqrt{ }$ & $\sqrt{ }$ & $\sqrt{ }$ & $\sqrt{ }$ \\
\hline
\end{tabular}




\begin{tabular}{|c|c|c|c|c|}
\hline \multirow{2}{*}{ Names } & \multicolumn{4}{|c|}{ Variables selected using regression method } \\
\hline & Force Enter & stepwise & Forward & Backward \\
\hline Increased Parking Costs for Drive Alones & $\sqrt{ }$ & $\sqrt{ }$ & $\sqrt{ }$ & $\sqrt{ }$ \\
\hline Other Parking Management Strategies & $\sqrt{ }$ & $\sqrt{ }$ & $\sqrt{ }$ & $\sqrt{ }$ \\
\hline Subsidized Parking for Ridesharers & $\sqrt{ }$ & & & \\
\hline Work at Home (TeleCommuting) & $\sqrt{ }$ & & & \\
\hline Work at Satellite Center (TeleCommuting) & $\sqrt{ }$ & & & \\
\hline 3/36 Compressed Work Week Schedule & $\sqrt{ }$ & & & \\
\hline 4/40 Compressed Work Week Schedule & $\sqrt{ }$ & & $\sqrt{ }$ & \\
\hline 9/80 Compressed Work Week Schedule & $\sqrt{ }$ & & & $\sqrt{ }$ \\
\hline Other Compressed Work Week Schedule & $\sqrt{ }$ & $\sqrt{ }$ & $\sqrt{ }$ & $\sqrt{ }$ \\
\hline Other Employee Benefits and Services & $\sqrt{ }$ & & & \\
\hline Drawings, Free Meals, Certificates, etc. & $\sqrt{ }$ & & & \\
\hline Company Owned/Leased Vanpools & $\sqrt{ }$ & & & \\
\hline On-Site Childcare Service & $\sqrt{ }$ & & & \\
\hline Other On-Site Services & $\sqrt{ }$ & & & \\
\hline Cafeteria, ATM's, Postal, Fitness Center & $\sqrt{ }$ & $\sqrt{ }$ & $\sqrt{ }$ & $\sqrt{ }$ \\
\hline Transit Information or Pass Sales & $\sqrt{ }$ & & & \\
\hline FACILITY_AMENITIES & $\sqrt{ }$ & & & $\sqrt{ }$ \\
\hline RIDE_HOME & $\sqrt{ }$ & $\sqrt{ }$ & $\sqrt{ }$ & $\sqrt{ }$ \\
\hline FLEXTIME & $\sqrt{ }$ & & & \\
\hline MARKETING & $\sqrt{ }$ & & & $\sqrt{ }$ \\
\hline RS MATCH & $\sqrt{ }$ & & & \\
\hline FINANCIAL & $\sqrt{ }$ & & & \\
\hline PARKMGT\$ & $\sqrt{ }$ & & $\sqrt{ }$ & \\
\hline TELE_GRP & $\sqrt{ }$ & & & \\
\hline COMPRESSED & $\sqrt{ }$ & $\sqrt{ }$ & $\sqrt{ }$ & $\sqrt{ }$ \\
\hline VANPOOL & $\sqrt{ }$ & & & $\sqrt{ }$ \\
\hline ONSITE & $\sqrt{ }$ & & & \\
\hline DIRECT_NONFINANCIAL & $\sqrt{ }$ & & & \\
\hline OTHER & $\sqrt{ }$ & & & \\
\hline COMMTAX & $\sqrt{ }$ & & & \\
\hline Vehicle trip rate & & $\sqrt{ }$ & $\sqrt{ }$ & $\sqrt{ }$ \\
\hline Total Employee trips & $\sqrt{ }$ & & & \\
\hline Total Vehicle trips & $\sqrt{ }$ & & & \\
\hline
\end{tabular}

Figures $5,6 \& 7$ show charts of the 'bin classification accuracy on moderate range of change in VTR', 'bin classification accuracy on full range of change in VTR' and the R-square values for the regression models and the corresponding neural network models built using the variable sets selected by the regression models. It can be seen from the table 4 that the number ranges are labeled below as "a1," "a2," "a3," etcetera. For example, the "a2" labeled bin covers a range of change in VTR from -7 to -4 and the "a5" labeled bin covers a range of change in VTR from 0 to 1 . Therefore, the bins "a2" through "a5" would cover the change in VTR range between -7 through +1 which can be considered as a moderate range.

In Figures 5, 6, and 7 below, for each methodology (i.e. forced enter, stepwise, forward, or backward), the models labeled as "linear model" specify the SPSS regression models built on the variables obtained from the specific methodologies and the models labeled as a "sequence on three numbers" (Input neurons - hidden neurons - output neurons) specify the architecture of the neural net model also built from those same variables. 


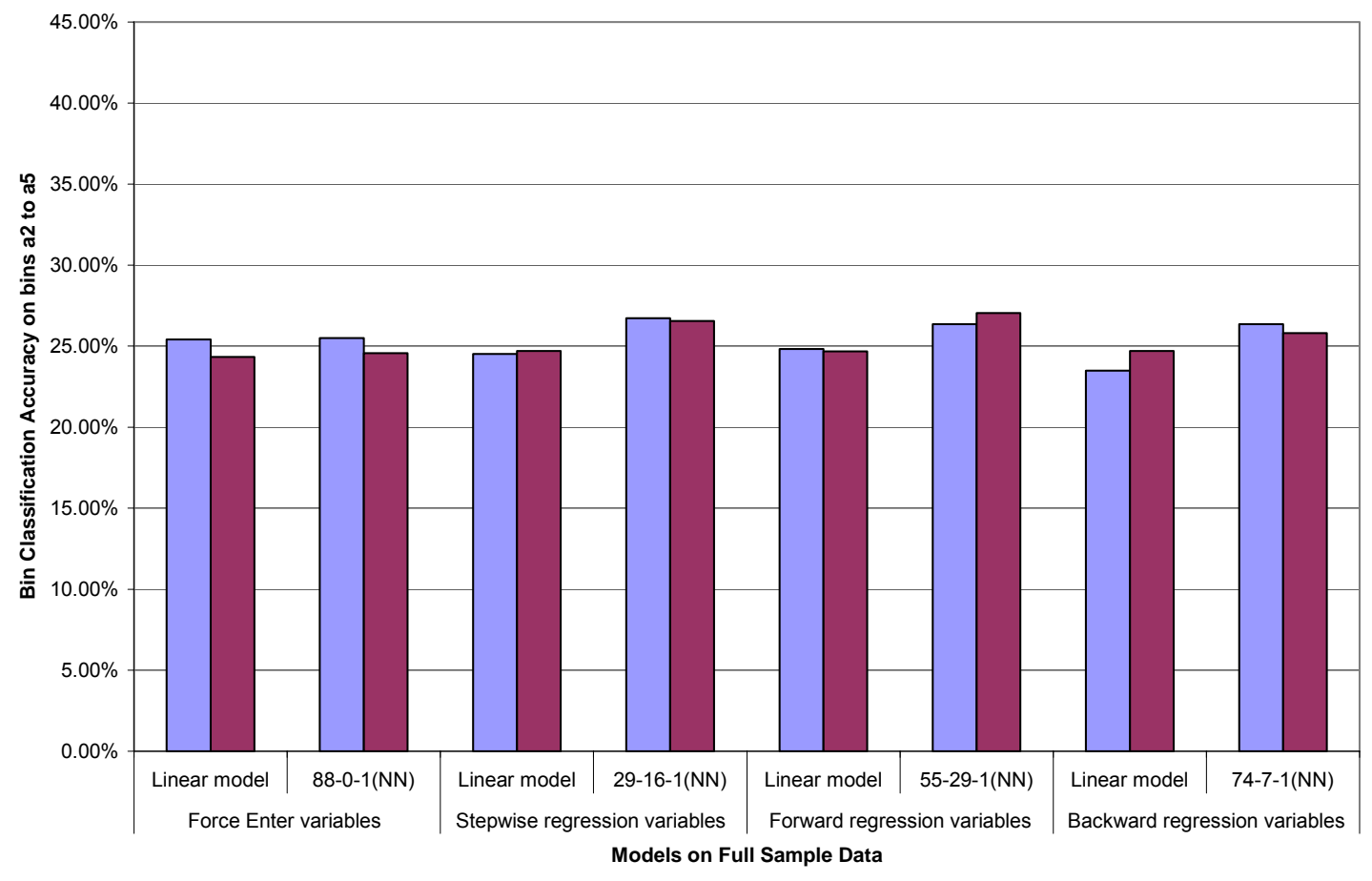

$\square$ Accuracy on Validation set $\square$ Accuracy on Training set

Figure 5: Bin Classification Accuracy on Moderate Range of change in VTR (a2 to a5) for validation \& training set (Different models on Full sample data)

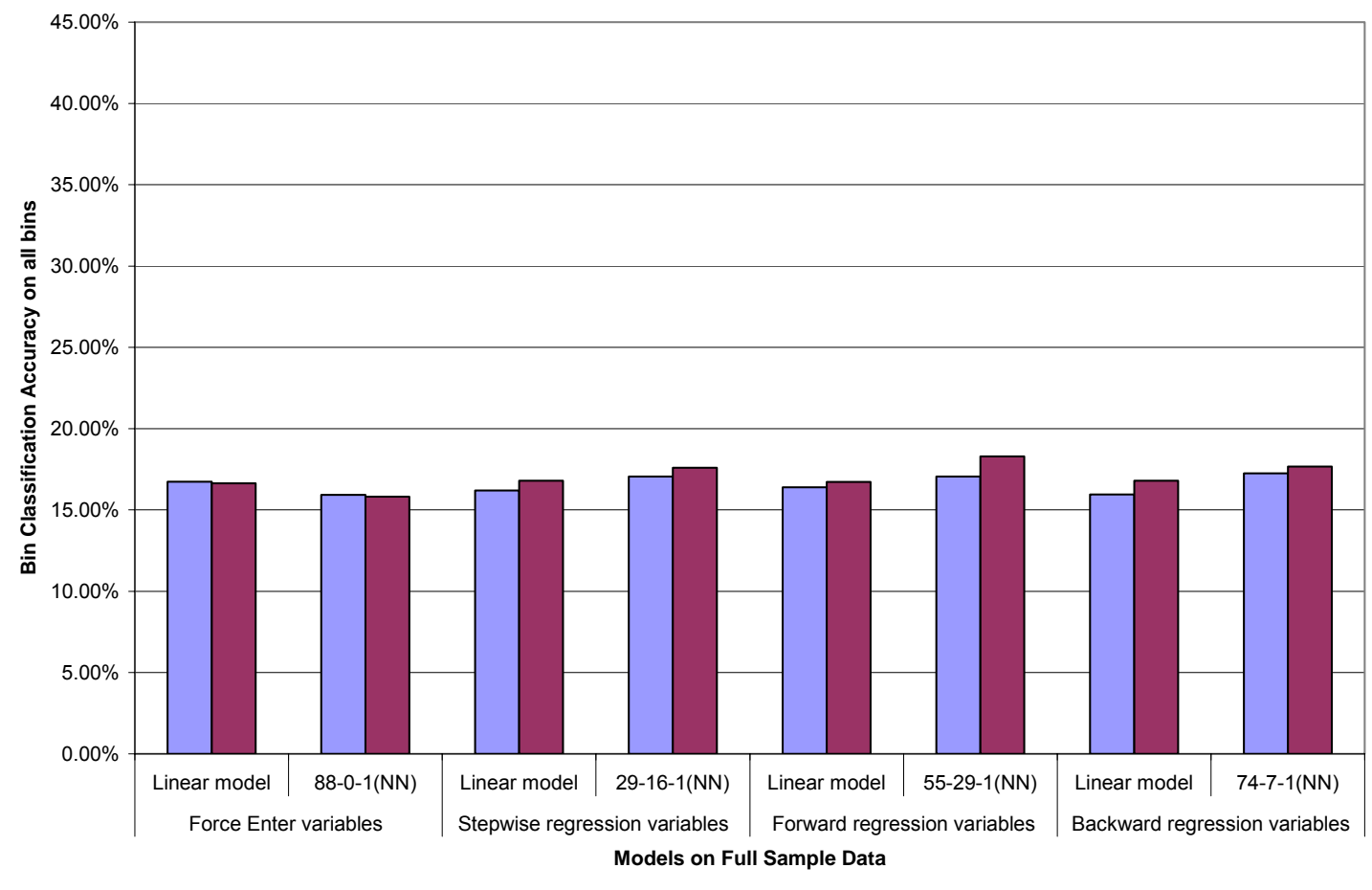

$\square$ Accuracy on Validation Set $\square$ Accuracy on Training Set

Figure 6: Bin Classification Accuracy on Full Range of change in VTR(all bins) for validation \& training set (Different models on Full sample data) 


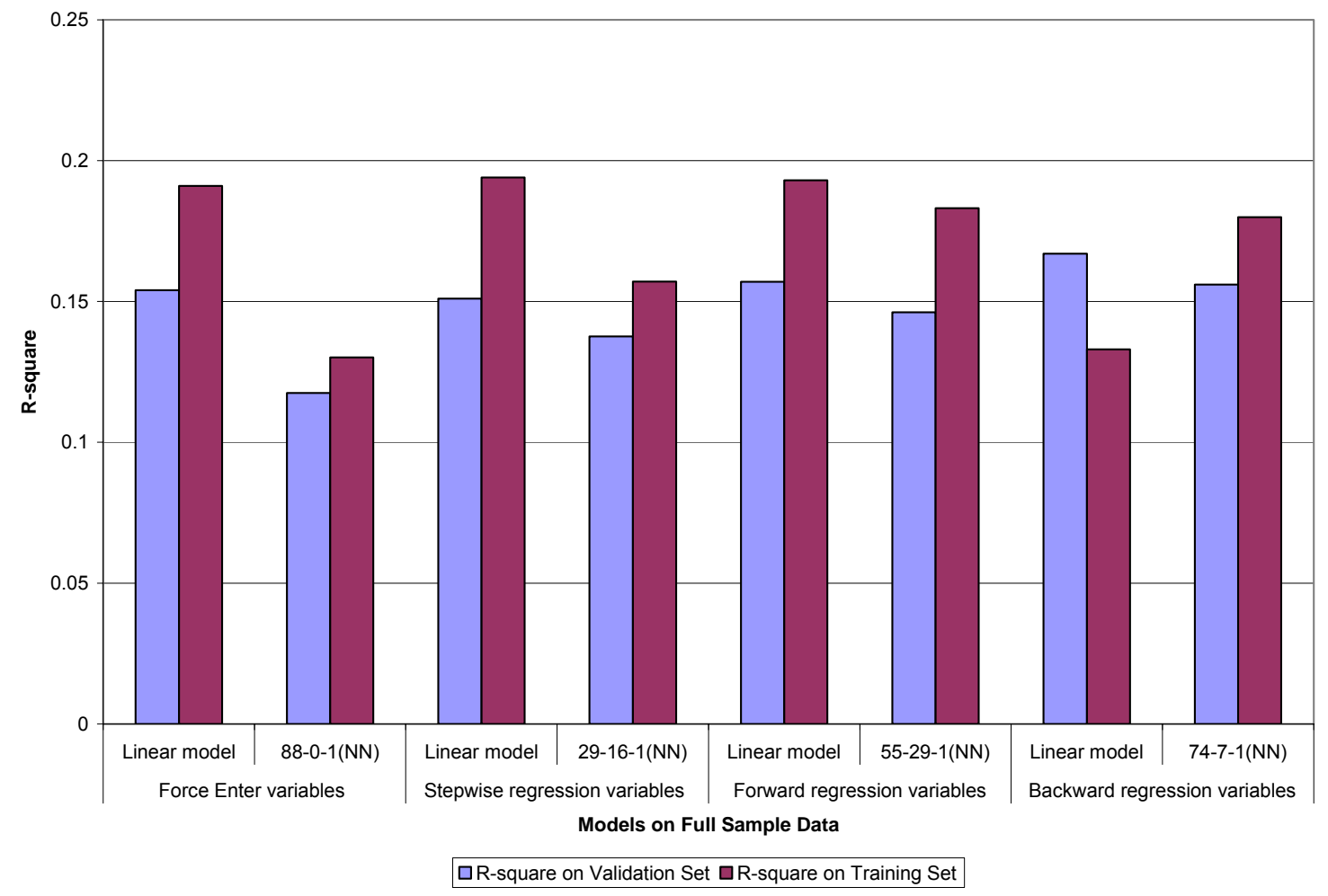

Figure 7: R-square on training and validation data (Different models on Full sample data)

It can be seen from the figures that the neural network model built using the stepwise regression variables yielded the best 'bin classification accuracy on moderate range of change in VTR', and the second best 'bin classification accuracy on full range of change in VTR'.

Table 6: Detailed accuracies for NN model on stepwise regression variables

\begin{tabular}{|c|c|c|c|c|c|c|c|c|c|c|}
\hline \multirow{2}{*}{ range } & \multirow{2}{*}{ \# of records } & \multicolumn{8}{|c|}{ Bin ranges over Change in Vehicle Trip Rate (Delta_VTR) } & \multirow{6}{*}{$\begin{array}{l}\text { Weighted } \\
\text { Avg on } \\
\text { a2 to a5 }\end{array}$} \\
\hline & & $>-7$ & {$[-7$ to -4$)$} & {$[-4$ to -2$)$} & [- 2 to -0$)$ & [-0 to 1) & [ 1 to 2.5 ) & [2.5 to 5 ) & $5>=$ & \\
\hline Bin Number & & a1 & a2 & a3 & a4 & a5 & a6 & a7 & a8 & \\
\hline Validation & 2537 & 347 & 286 & 356 & 423 & 237 & 264 & 262 & 362 & \\
\hline Training & 22922 & 3087 & 2969 & 2943 & 3988 & 2155 & 2260 & 2612 & 2908 & \\
\hline & & & & & & & & & & \\
\hline Exact Validation & $17.07 \%$ & $4.61 \%$ & $17.48 \%$ & $27.25 \%$ & $40.43 \%$ & $12.66 \%$ & $15.53 \%$ & $9.16 \%$ & $1.10 \%$ & $26.73 \%$ \\
\hline Exact Training & $17.59 \%$ & $5.54 \%$ & $18.22 \%$ & $25.45 \%$ & $39.77 \%$ & $15.03 \%$ & $15.58 \%$ & $9.49 \%$ & $2.10 \%$ & \\
\hline One-off Validation & $47.22 \%$ & $28.82 \%$ & $45.80 \%$ & $72.75 \%$ & $70.45 \%$ & $64.98 \%$ & $35.23 \%$ & $30.53 \%$ & $22.93 \%$ & $64.67 \%$ \\
\hline One-Off Training & $47.89 \%$ & $29.87 \%$ & $44.43 \%$ & $75.03 \%$ & $72.57 \%$ & $68.12 \%$ & $37.61 \%$ & $27.41 \%$ & $20.67 \%$ & \\
\hline
\end{tabular}

Table 6 explains how accuracy was verified on a bin by bin basis. Each of the predicted Delta_VTR obtained from the neural net and the regression models were compared against the real change in vehicle trip rate obtained from the data sets. Each of these Delta_VTR values were coded into bins using the ranges as shown in Table 4 and the results were cross tabulated to ascertain the accuracy of the predicted values versus the real values. The bin accuracy system was explained in the previous section, but for 
clarity, the designation "Exact Validation" refers to the accuracy on training set where the predicted bins exactly matched the actual bins. "One-off Training" refers to the accuracy on training set where the predicted bin matched the actual bin or one of the adjacent bins. "Exact Validation" refers to the accuracy on validation set where the predicted bins exactly matched the actual bins and "One-off Validation" refers to the accuracy on validation set where the predicted bin matched the actual bin or one of the adjacent bins. For example, the model shown in Table 6 accurately predicted 72.75 percent of the records showing a reduction from 0 to -7 vehicle trips.

In Figure 8 which shows the scatter plot for this model on the validation set, the upper right quadrant shows positive predicted values that matched with positive actual values and the lower left quadrant shows negative predicted values that matched with the negative actual values. The upper left and lower right represent predicted values which were mismatched to the actual values with respect to the sign (i.e., negative or positive) value.

Actual Delta_VTR Vs. Predict Delta_VTR

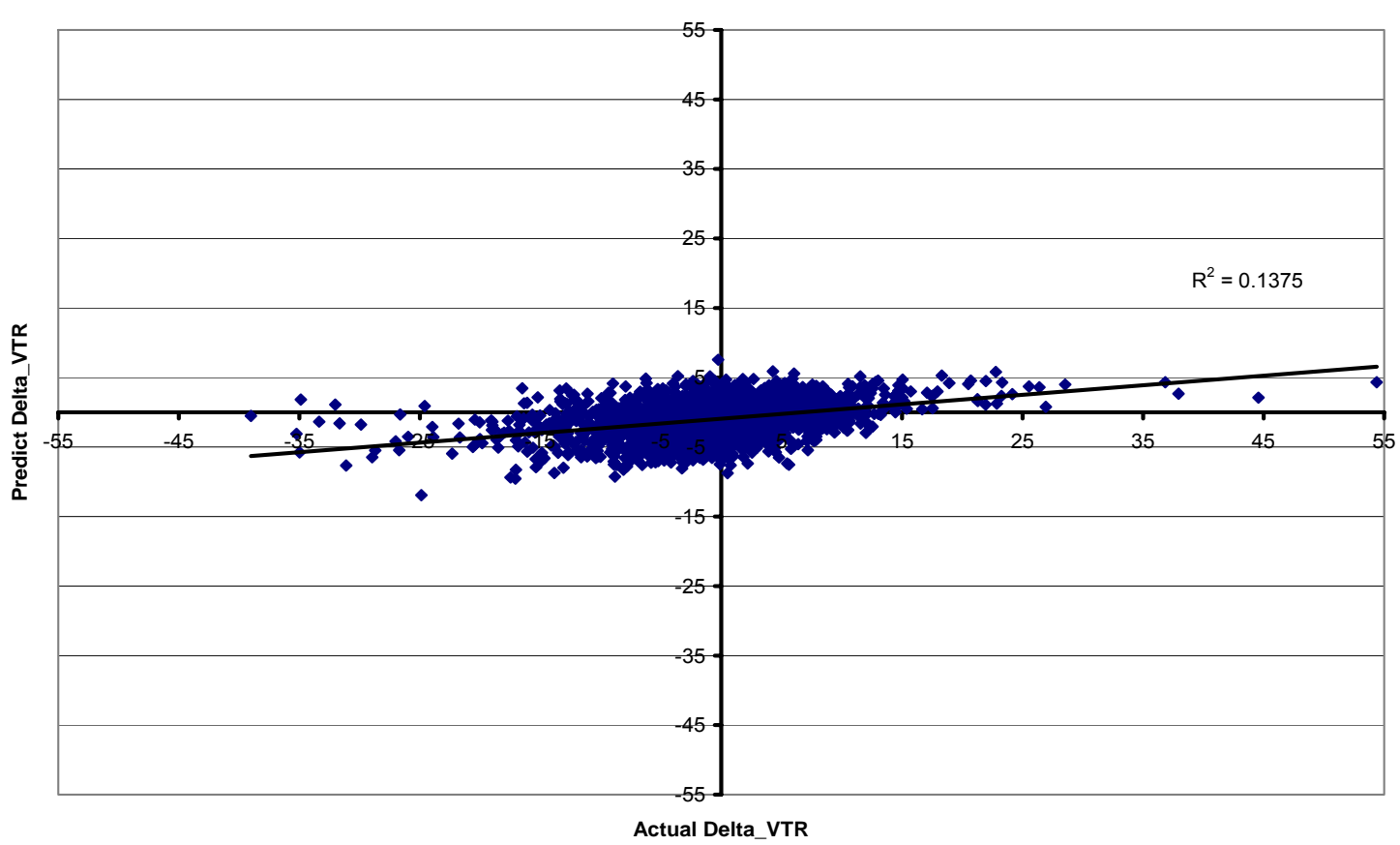

Figure 8: Scatter plot for NN model on stepwise regression variables 


\section{Phase II : Los Angeles Over-Sampled Data}

To get better accuracies over the required range on change in VTR, the examples in some bins were over-sampled and others sub-sampled. Table 7 shows the new number of examples in training set due to over-sampling and sub-sampling.

Table 7: Los Angeles Over-Sampled Data - No. of Records in bins

\begin{tabular}{|l|c|c|c|c|c|c|c|c|c|}
\hline \multirow{2}{*}{ range } & \multirow{2}{*}{ \# of records } & \multicolumn{8}{|c|}{ Bin ranges over Change in Vehicle Trip Rate (Delta_VTR) } \\
\cline { 3 - 10 } & & $\mathbf{7 - 7}$ & [- 7 to - 4) & [- - to - 2) & [- 2 to -0) & [-0 to 1) & [ 1 to 2.5) & [2.5 to 5) & 5 >= \\
\hline Bin Number & & $\mathbf{a 1}$ & $\mathbf{a 2}$ & $\mathbf{a 3}$ & $\mathbf{a 4}$ & $\mathbf{a 5}$ & $\mathbf{a 6}$ & $\mathbf{a 7}$ & a8 \\
Validation & $\mathbf{2 5 3 7}$ & 347 & 286 & 356 & 423 & 237 & 264 & 262 & 362 \\
Training & $\mathbf{3 5 2 2 7}$ & 4564 & 2534 & 6629 & 11500 & 4000 & 3320 & 1324 & 1356 \\
\hline
\end{tabular}

The neural network models were built on variables selected by forward and stepwise regression. Additionally, the costs associated with each incentive were added for each of the respective stepwise incentives and a neural network model was constructed from these cost-modified variables.

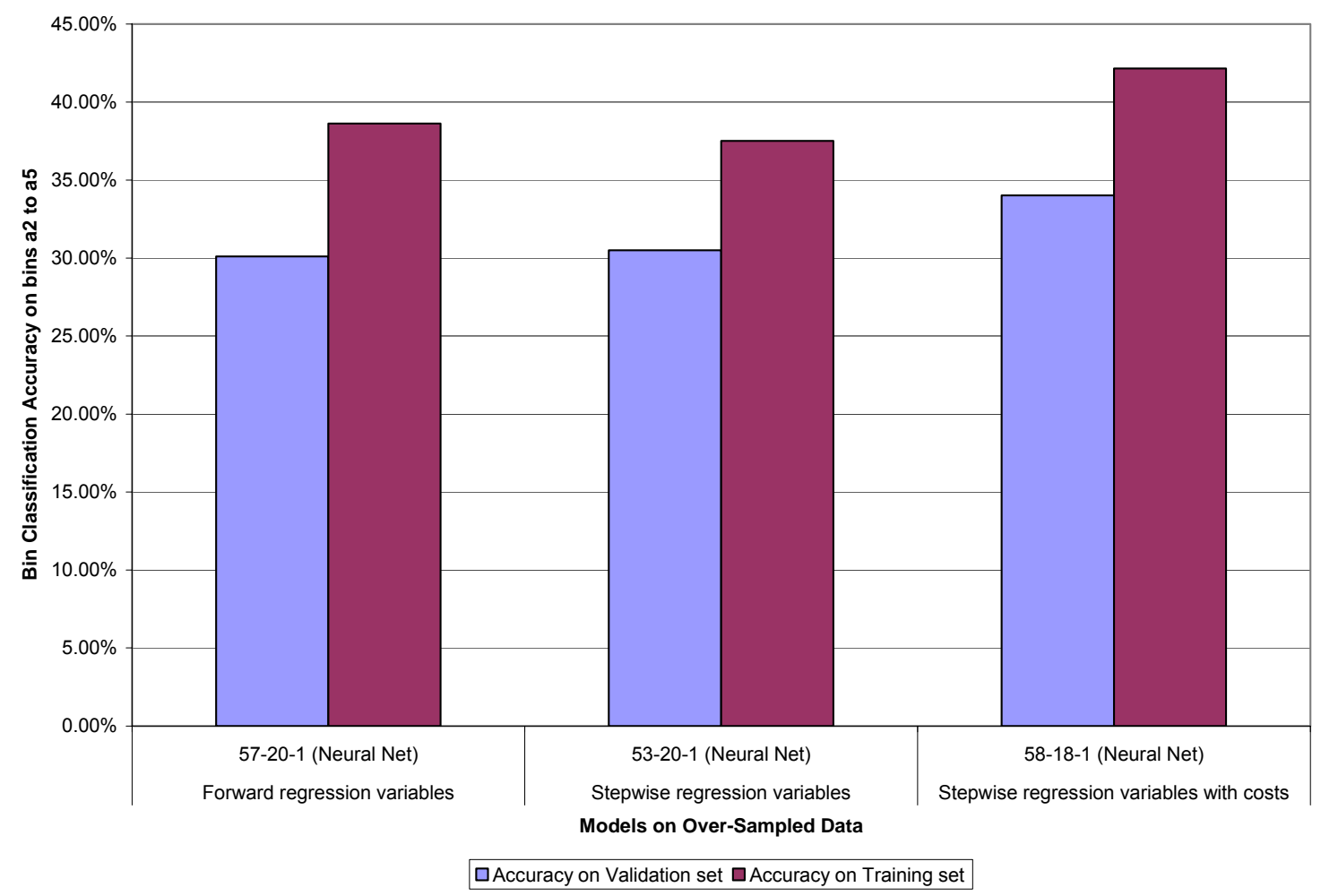

Figure 9: Bin Classification Accuracy on Moderate Range of change in VTR (a2 to a5) for validation \& training set (Different models on Over-sampled data) 


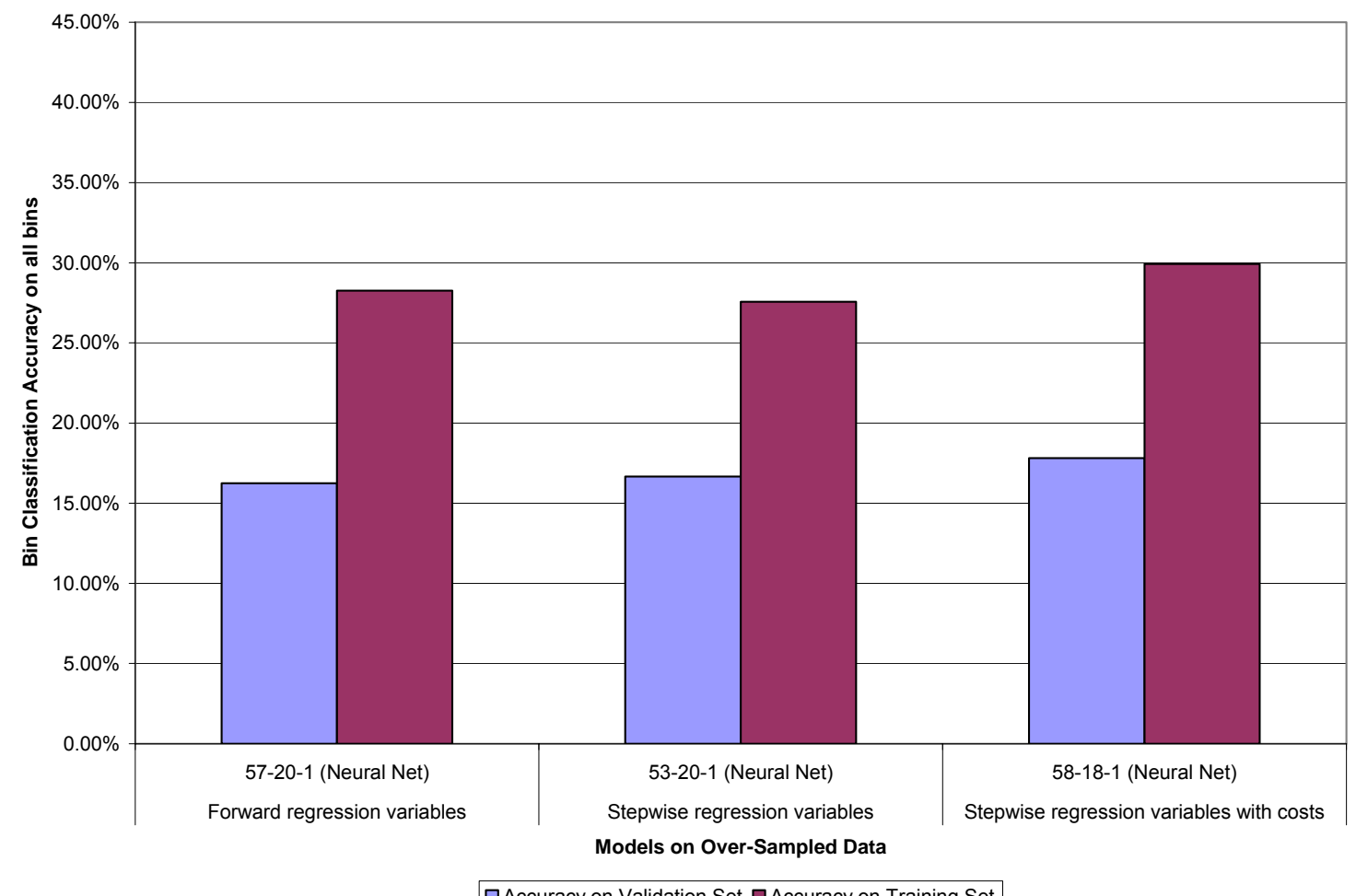

$\square$ Accuracy on Validation Set $\square$ Accuracy on Training Set

Figure 10: Bin Classification Accuracy on Full Range of change in VTR (all bins) for validation \& training set (Different models on Over-sampled data)

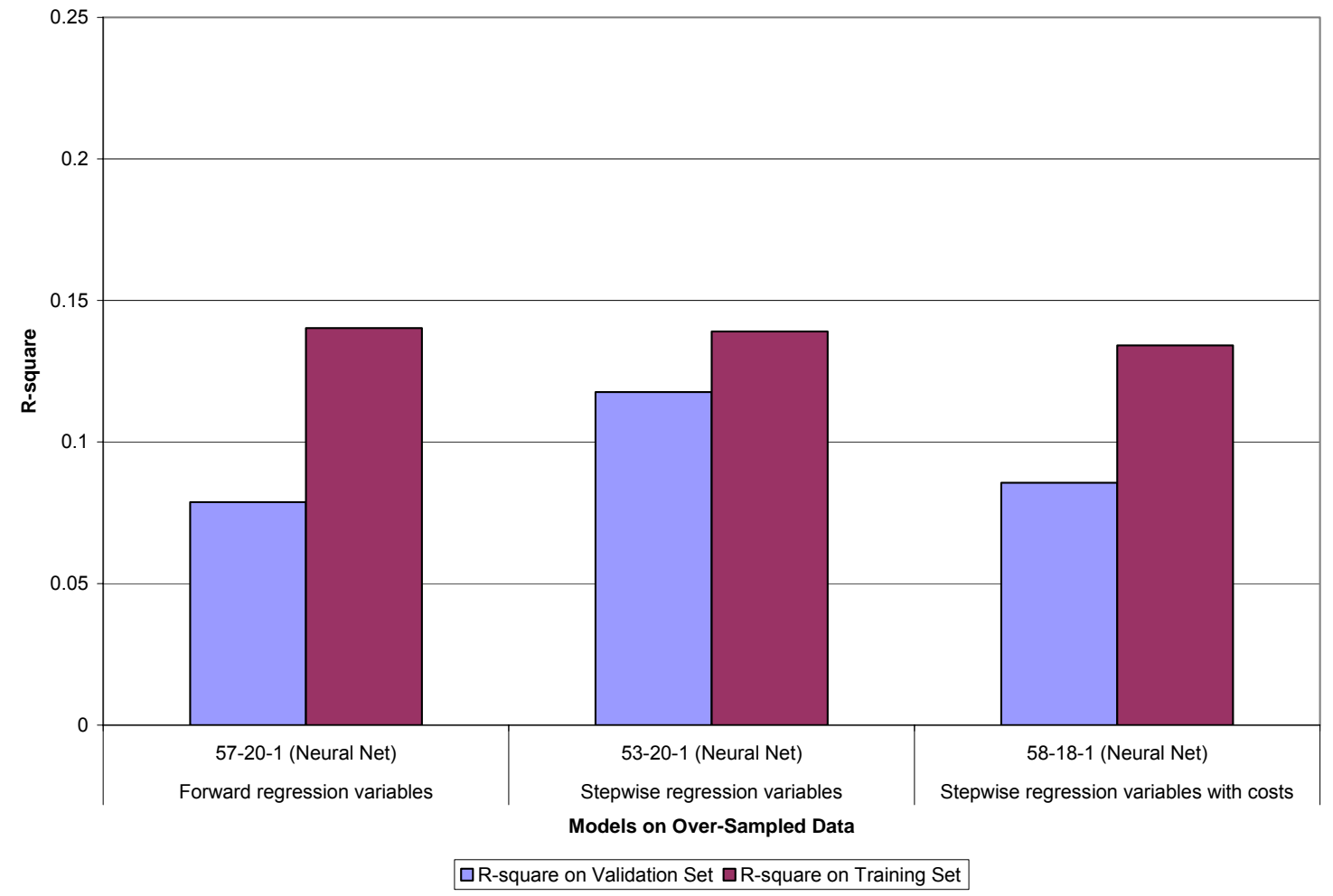

Figure 11: R-square for validation \& training set (Different models on Over-sampled data) 
As can be seen from the figures, for all the models the 'bin classification accuracy on moderate range of change in VTR' has been greatly improved, with little change in total accuracy. However, the R-square value on the validation set has gone down drastically. The model which got the best of three measures was the one constructed with stepwise regression attributes.

But it can be seen from the scatter plot of this model in Figure 12, that for many of the records with positive change in VTR, the model predicted negative change in VTR. The best model should predict both negative as well as positive changes in VTR with a high degree of accuracy. However, this particular model was biased towards predicting negative changes in VTR, and hence fails to achieve the required goal.

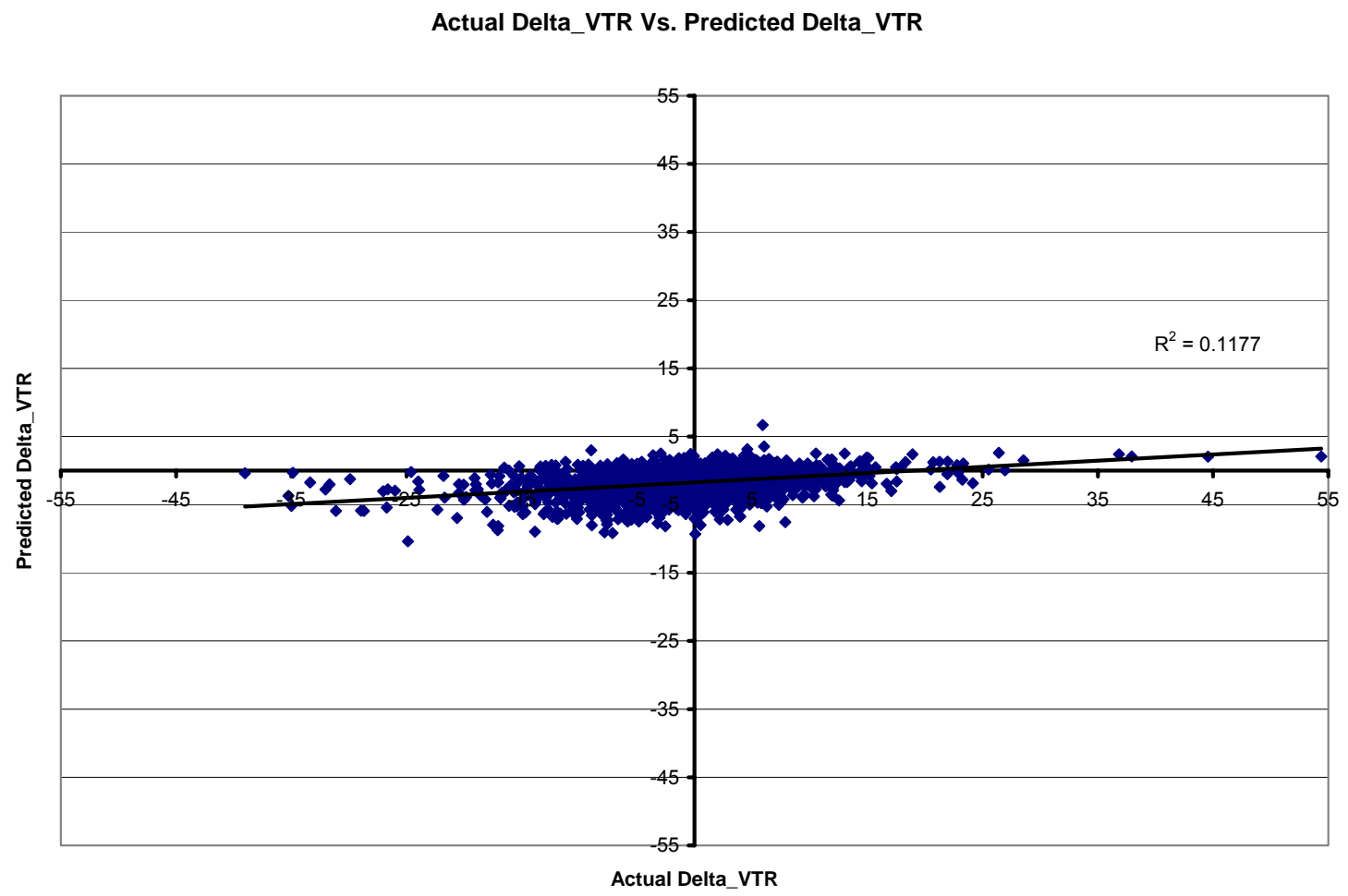

Figure 12: Scatter plot for stepwise linear regression model 


\section{Phase III: Los Angeles Data - records with no incentives removed}

Some of the cases in the data sets did not have any incentives implemented. Such records would not be helpful in trying to find the effectiveness of incentives on the reduction in vehicle trip rate and were removed from the two datasets respectively, with 18,140 records remaining in the final data set.

Table 8: Los Angeles data with records with no Incentives Removed - No. of Records in bins

\begin{tabular}{|l|c|c|c|c|c|c|c|c|c|}
\hline \multirow{2}{*}{ range } & \multirow{2}{*}{ \# of records } & \multicolumn{6}{|c|}{ Bin ranges over Change in Vehicle Trip Rate (Delta_VTR) } \\
\cline { 3 - 10 } & & $\mathbf{> - 7}$ & [- - t to - 4) & [- - 4 to - 2) & [- 2 to -0) & [-0 to 1) & [1 to 2.5) & [2.5 to 5) & $\mathbf{5}>=$ \\
\hline Bin Number & & $\mathbf{a 1}$ & $\mathbf{a 2}$ & $\mathbf{a 3}$ & $\mathbf{a 4}$ & $\mathbf{a 5}$ & $\mathbf{a 6}$ & $\mathbf{a 7}$ & $\mathbf{a 8}$ \\
Validation & $\mathbf{1 8 3 8}$ & 292 & 220 & 261 & 290 & 173 & 157 & 186 & 259 \\
Training & $\mathbf{1 6 3 0 2}$ & 2543 & 2310 & 2192 & 2635 & 1414 & 1479 & 1714 & 2015 \\
\hline
\end{tabular}

Several models were built on the data with these records, but with different variable sets.

For some models, the costs associated with each incentive were added into the dataset. Because the model with stepwise regression variables gave the best results of the full sample data, it was once again used on this data. The variable set selected by the model is shown in Table 9.

Table 9: Variables stepwise regression--data with associated incentive costs

\begin{tabular}{|l|l|}
\hline \multicolumn{1}{|c|}{ Variable } & \multicolumn{1}{c|}{ Description } \\
\hline VTR_CUTR & Vehicle trip rate \\
TRANSIT & Share employees commuting using transit \\
BMM & Posted Materials (Marketing) \\
TELECOMMUTE & Share employees tele-commuting \\
CWW440 & Share of 4/40 CWW days off \\
BIKE & Share employees commuting using bike \\
ISS & Cafeteria, ATM's, Postal, Fitness Center \\
DW4 & 4/40 Compressed Work Week Schedule \\
BGT_DOLLAR_AMT & Taxi Guaranteed Return Trip amount \\
CWW336 & Share of employees on 3/36 CWW \\
TARGET_AVR & Target AVR required (3 zones) \\
BGE & Emergencies Guaranteed Return Trip \\
DNT & Additional Time Off with Pay \\
BMO & Other Marketing Elements \\
BUS & Share employees commuting in bus \\
CAR2 & Share employees commuting two together \\
DFT_DOLLAR_AMT & On-Going Transit Subsidies amount \\
RS_MATCH & Combined ride-share match \\
BMS & Special Interest Club (Biking, Walking) \\
BGC & Company Vehicle Guaranteed Return Trip \\
BGR_DOLLAR_AMT & Rental Car Guaranteed Return Trip amount \\
BMR & Company Recognition (Marketing) \\
DPC & Increased Parking Costs for Drive Alones \\
DPO & Other Parking Management Strategies \\
IST_DOLLAR_AMT & Transit Information or Pass Sales amount \\
BGT & Taxi Guaranteed Return Trip \\
DW4_DOLLAR_AMT & 4/40 Compressed Work Week Schedule amount \\
BGO & Other Guaranteed Return Trip Program \\
BFR & Bike Racks and Bike Lockers \\
BFO & Other Facility Improvements \\
BGU & Unscheduled Overtime Guaranteed Return \\
CAR4 & Share employees commuting four together \\
&
\end{tabular}




\begin{tabular}{|l|l|}
\hline \multicolumn{1}{|c|}{ Variable } & \multicolumn{1}{c|}{ Description } \\
\hline BMN & New Hire Orientation (Marketing) \\
DWO & Other Compressed Work Week Schedule \\
COMPRESSED & Combined compressed WW \\
CWW980 & Share of 9/80 CWW days off \\
\hline
\end{tabular}

A neural network model was built on the dataset containing the variables selected by the above regression model. Also a neural network model was built on variables selected by the stepwise regression on the full sample data with respective incentive costs added.

To reduce the complexity of the model, all of the individual incentives were removed and a simple model was built on the data containing only the worksite characteristics like mode-splits and the grouped incentives.

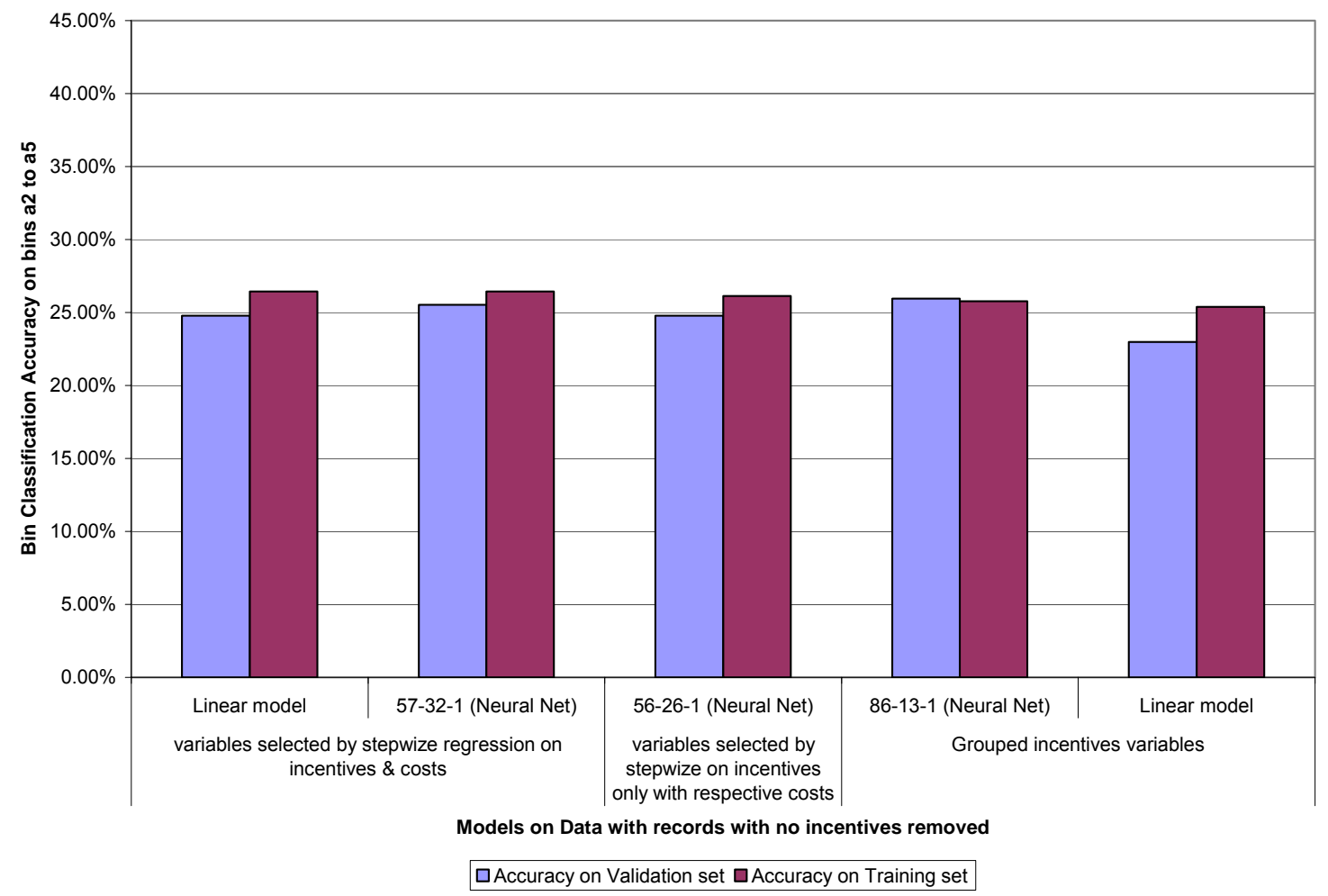

Figure 13: Bin Classification Accuracy on Moderate Range of change in VTR (a2 to a5) for validation \& training set (Different models on data - records with no incentives removed) 


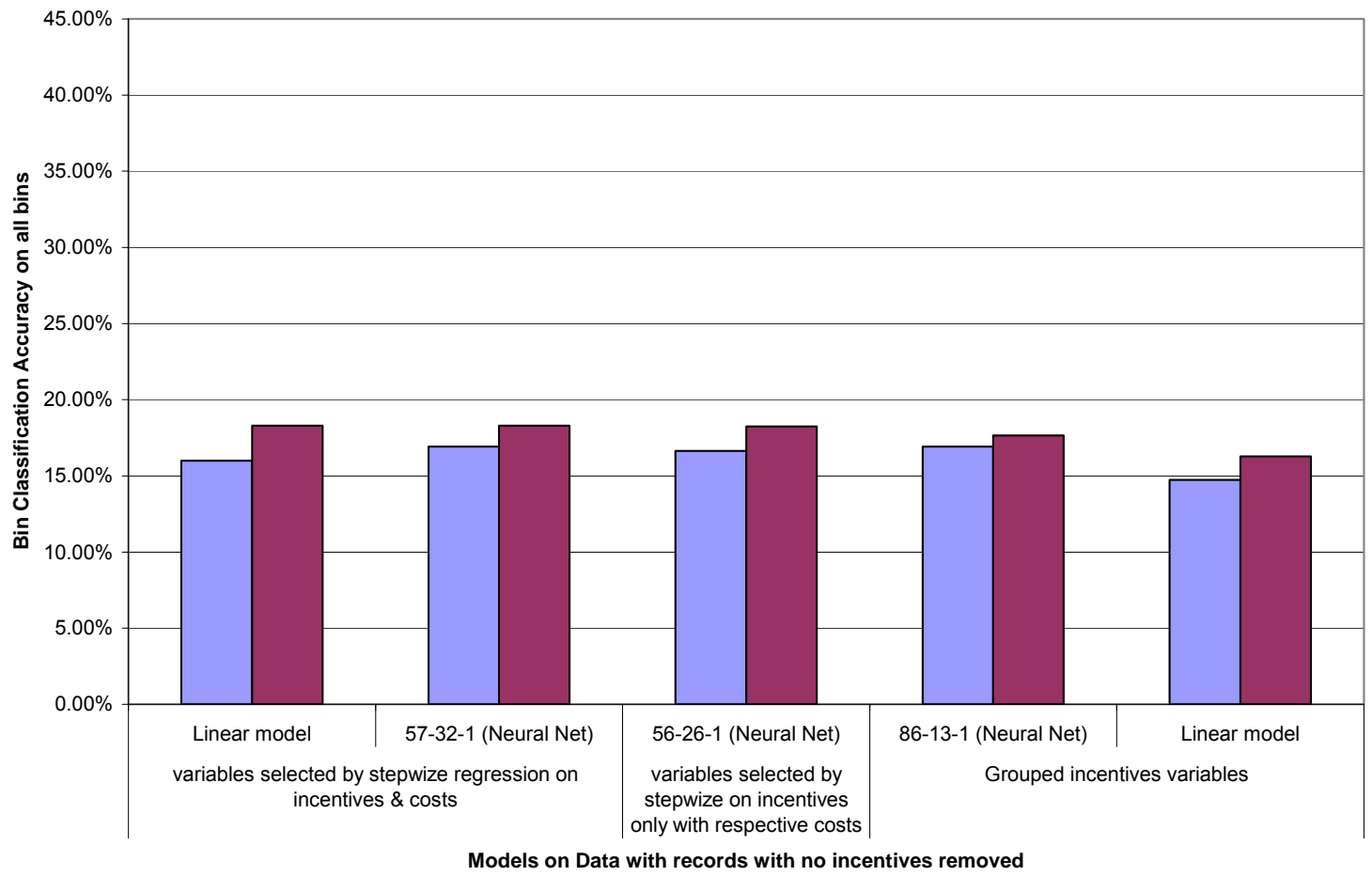

$\square$ Accuracy on Validation Set $\square$ Accuracy on Training Set

Figure 14: Bin Classification Accuracy on Full Range of change in VTR (all bins) for validation \& training set (Different models on data - records with no incentives removed)

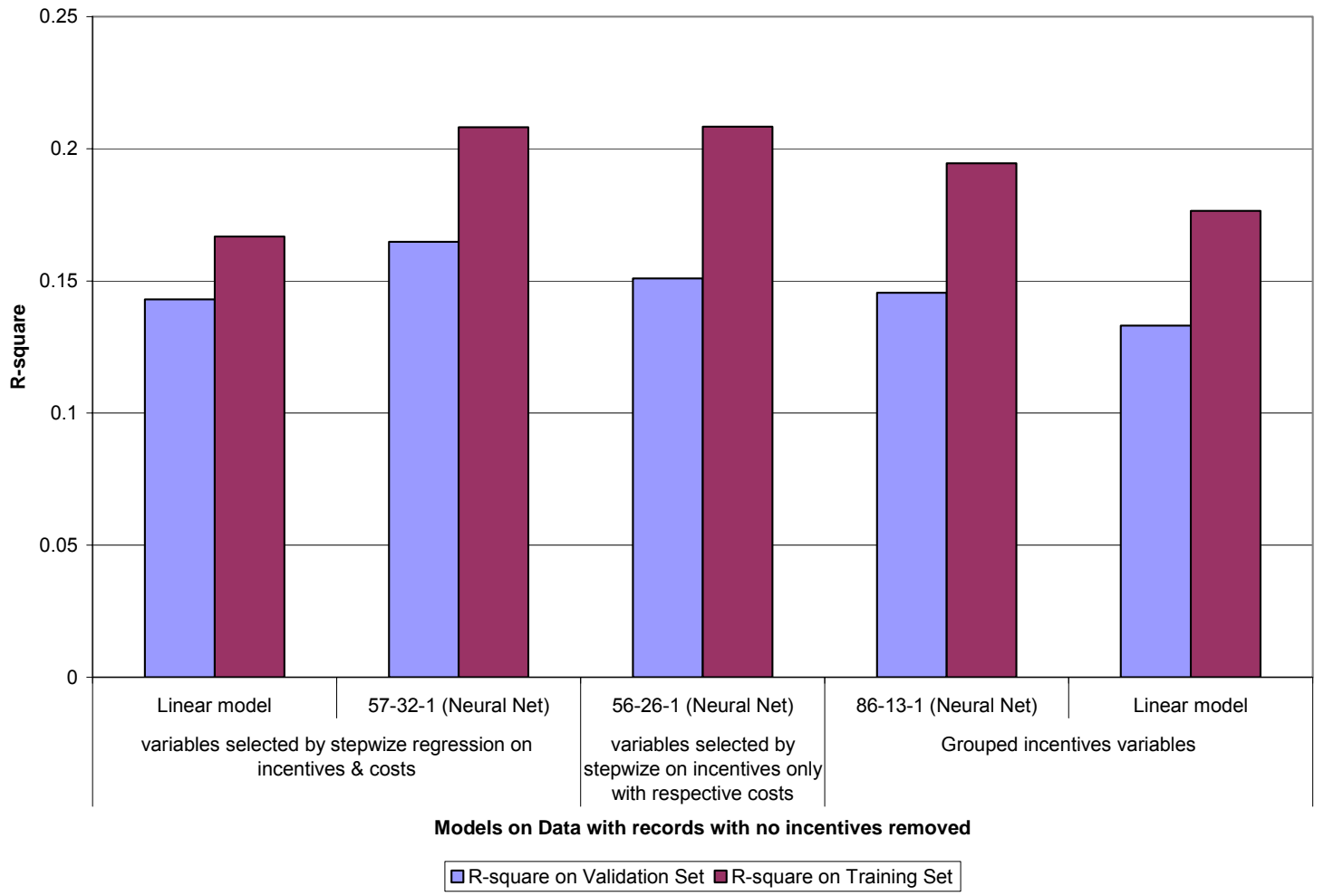

Figure 15: R-square for validation \& training set (Different models on data - records with no incentives removed) 
It can be seen from Figures 13 and 14, that the two neural network models, one built using the stepwise regression variables on incentives and costs (M1) and the other built on grouped incentives variables (M2) were two frontrunners for the best model. The bin accuracies for this model are shown in Table 10.

Table 10: Detailed accuracies on bins

\begin{tabular}{|c|c|c|c|c|c|c|c|c|c|c|c|c|}
\hline & \multirow{2}{*}{ range } & \multirow{2}{*}{$\begin{array}{c}\text { \# of } \\
\text { records }\end{array}$} & \multicolumn{8}{|c|}{ Bin ranges over Change in Vehicle Trip Rate (Delta_VTR) } & \multirow{4}{*}{$\begin{array}{l}\text { Weighted } \\
\text { Avg on } \\
\text { a2 to a5 }\end{array}$} & \multirow[b]{3}{*}{ R-square } \\
\hline & & & $>-7$ & {$[-7$ to -4$)$} & {$[-4$ to -2$)$} & {$[-2$ to -0$)$} & [-0 to 1$)$ & [ 1 to 2.5 ) & [2.5 to 5) & $5>=$ & & \\
\hline & \begin{tabular}{|l} 
Bin Number \\
Validation \\
Training \\
\end{tabular} & $\begin{array}{c}1838 \\
16302\end{array}$ & $\begin{array}{c}\mathbf{a 1} \\
292 \\
2543 \\
\end{array}$ & $\begin{array}{c}\mathbf{a 2} \\
220 \\
2310 \\
\end{array}$ & $\begin{array}{c}\text { a3 } \\
261 \\
2192 \\
\end{array}$ & $\begin{array}{c}\mathbf{A 4} \\
290 \\
2635 \\
\end{array}$ & $\begin{array}{c}\mathbf{a 5} \\
173 \\
1414 \\
\end{array}$ & $\begin{array}{c}\mathbf{a 6} \\
157 \\
1479 \\
\end{array}$ & $\begin{array}{c}\mathbf{a 7} \\
186 \\
1714 \\
\end{array}$ & $\begin{array}{c}\mathbf{a 8} \\
259 \\
2015 \\
\end{array}$ & & \\
\hline & & & & & & & & & & & & \\
\hline M1 & $\begin{array}{l}\text { Exact Validation } \\
\text { Exact Training } \\
\text { One-off Validation } \\
\text { One-Off Training }\end{array}$ & $\begin{array}{l}16.92 \% \\
18.30 \% \\
47.82 \% \\
49.53 \%\end{array}$ & \begin{tabular}{|c|}
$7.19 \%$ \\
$7.75 \%$ \\
$31.85 \%$ \\
$37.48 \%$
\end{tabular} & $\begin{array}{l}19.55 \% \\
22.68 \% \\
54.09 \% \\
54.37 \%\end{array}$ & $\begin{array}{l}27.59 \% \\
30.34 \% \\
77.78 \% \\
76.05 \%\end{array}$ & $\begin{array}{l}35.52 \% \\
33.02 \% \\
67.24 \% \\
68.92 \%\end{array}$ & $\begin{array}{l}13.29 \% \\
14.29 \% \\
56.65 \% \\
58.77 \%\end{array}$ & $\begin{array}{l}12.10 \% \\
12.64 \% \\
36.94 \% \\
35.36 \%\end{array}$ & $\begin{array}{c}6.99 \% \\
9.33 \% \\
27.42 \% \\
27.19 \%\end{array}$ & \begin{tabular}{|c|}
$6.56 \%$ \\
$8.83 \%$ \\
$23.94 \%$ \\
$27.89 \%$
\end{tabular} & $\begin{array}{l}25.53 \% \\
65.15 \%\end{array}$ & $\begin{array}{l}0.1649 \\
0.2081\end{array}$ \\
\hline M2 & $\begin{array}{l}\text { Exact Validation } \\
\text { Exact Training } \\
\text { One-off Validation } \\
\text { One-Off Training }\end{array}$ & $\begin{array}{l}16.92 \% \\
17.65 \% \\
45.48 \% \\
48.77 \%\end{array}$ & \begin{tabular}{|c|}
$4.11 \%$ \\
$5.82 \%$ \\
$27.40 \%$ \\
$31.97 \%$
\end{tabular} & $\begin{array}{l}19.09 \% \\
20.13 \% \\
50.91 \% \\
52.03 \%\end{array}$ & $\begin{array}{l}27.59 \% \\
30.93 \% \\
75.48 \% \\
78.88 \%\end{array}$ & $\begin{array}{l}36.21 \% \\
34.04 \% \\
71.72 \% \\
72.87 \%\end{array}$ & $\begin{array}{l}15.03 \% \\
11.60 \% \\
51.45 \% \\
57.99 \%\end{array}$ & $\begin{array}{l}11.46 \% \\
12.04 \% \\
32.48 \% \\
31.78 \%\end{array}$ & $\begin{array}{c}9.14 \% \\
9.63 \% \\
23.12 \% \\
25.73 \%\end{array}$ & $\begin{array}{c}7.34 \% \\
9.03 \% \\
21.62 \% \\
27.54 \%\end{array}$ & $\begin{array}{l}25.95 \% \\
64.19 \%\end{array}$ & $\begin{array}{l}0.1456 \\
0.1945\end{array}$ \\
\hline
\end{tabular}

It can be seen from table 10, that both the models have same 'bin classification accuracy on full range of change in VTR'. The grouped incentive model has better 'bin classification accuracy on moderate range of change in VTR' than the other model with less R-square value. It can also be seen that the grouped incentive model was able to get better bin accuracies on positive range of change in VTR.

The scatter plots for both models on the validation set are shown in Figures 16 and 17. 
Actual Delta VTR Vs. Predicted Delta VTR

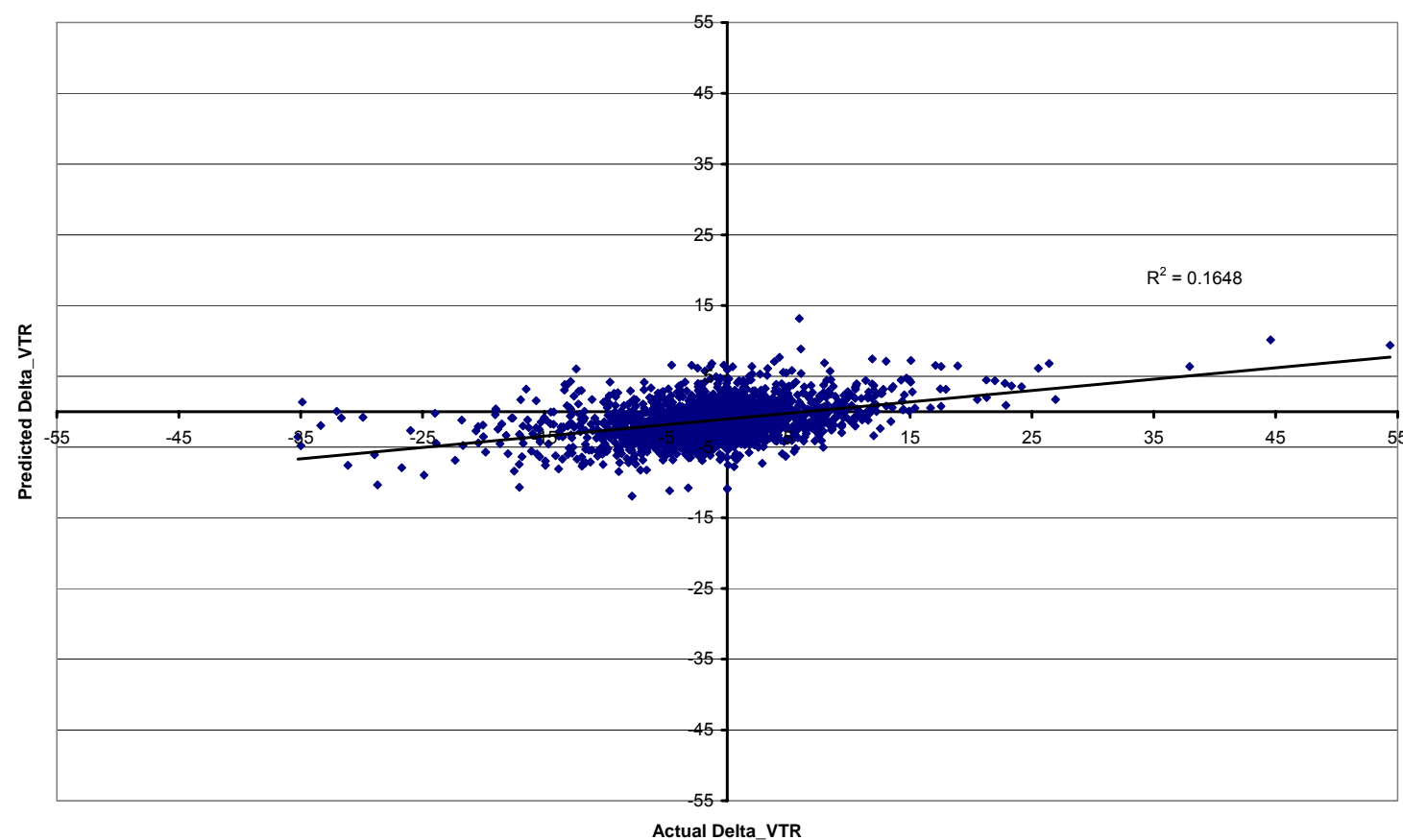

Figure 16 : Scatter plot for $\mathrm{NN}$ model built on stepwise regression variable Actual Delta_VTR Vs. Predicted Delta_VTR

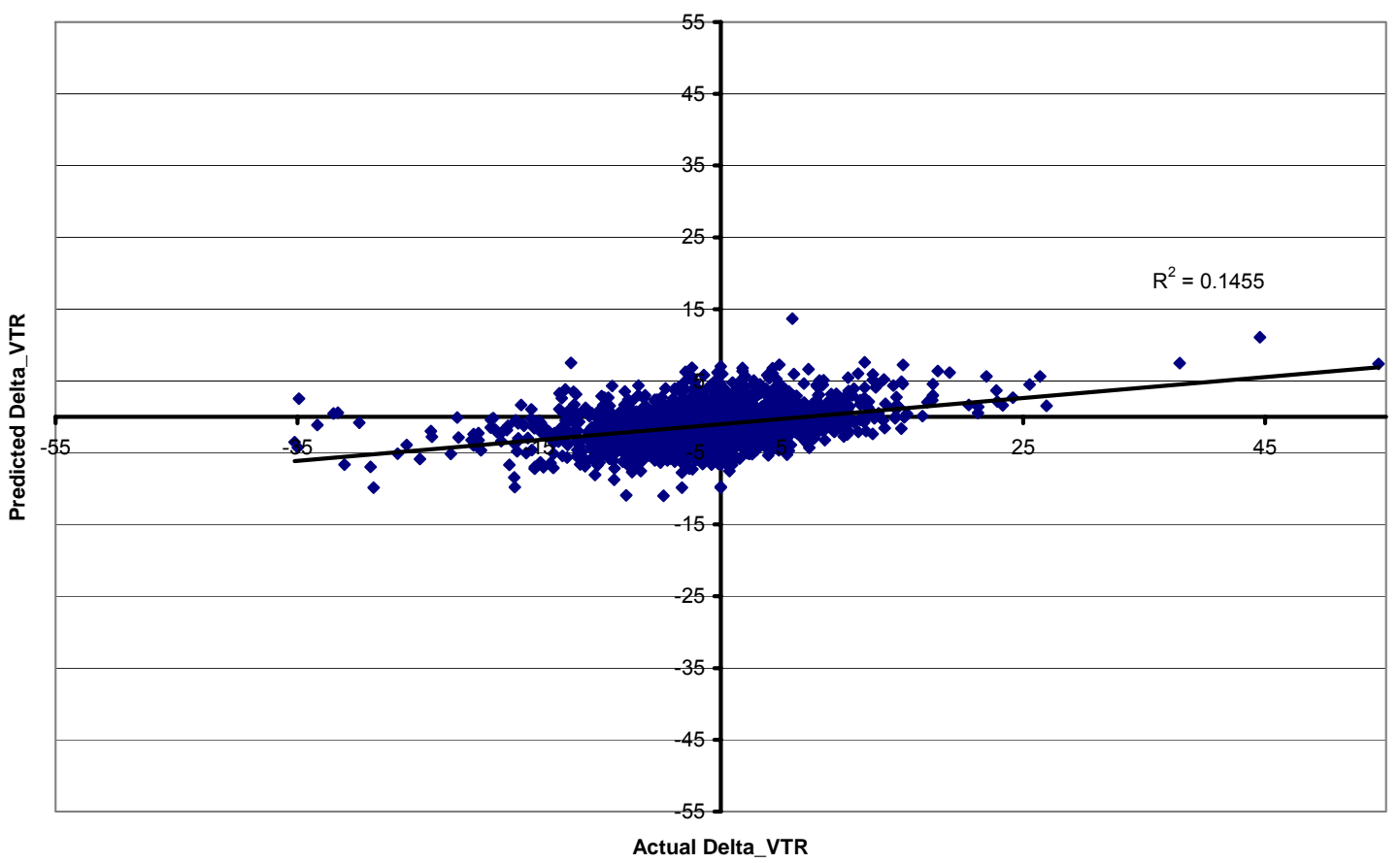

Figure 17: Scatter plot for NN model built on Grouped incentive variable 


\section{Phase IV: Los Angeles Data - records with no incentives \& outliers removed}

The dataset contained some records with very large changes in the VTR in both directions. It was felt that such large changes might not be attributed to the incentives plans implemented by the companies but rather to other non recorded measures. So the records with change in VTR less than -15.2 and greater than 12.5 were considered as outliers and were removed from the training and validation data. The reason for the selection of these cut-off values for change in VTR was that, these values that were outside of the normal distribution of the dataset. In order to obtain these values, SPSS 11.1 was used to identify values in the dataset which were greater or less than three standard deviations of the mean. This resulted in 790 records being dropped from the validation set and 7,432 dropped from the training set.

Table 11: Los Angeles data with records no incentives \& outliers removed- - No. of Records in bins

\begin{tabular}{|l|c|c|c|c|c|c|c|c|c|}
\hline \multirow{2}{*}{ range } & \multirow{2}{*}{ \# of records } & \multicolumn{8}{|c|}{ Bin ranges over Change in Vehicle Trip Rate (Delta_VTR) } \\
\cline { 3 - 10 } & & [- 15.2 to - 7) & [- 7 to - 4) & [- - to - 2) & [- 2 to -0) & [-0 to 1) & [1 to 2.5) & [2.5 to 5) & [5 to 12.5) \\
\hline Bin Number & & $\mathbf{a 1}$ & $\mathbf{a 2}$ & $\mathbf{a 3}$ & $\mathbf{a 4}$ & $\mathbf{a 5}$ & $\mathbf{a 6}$ & $\mathbf{a 7}$ & $\mathbf{a 8}$ \\
Validation & $\mathbf{1 7 3 8}$ & 236 & 220 & 261 & 290 & 173 & 157 & 186 & 215 \\
Training & $\mathbf{1 5 4 9 0}$ & 2103 & 2310 & 2192 & 2635 & 1414 & 1479 & 1714 & 1643 \\
\hline
\end{tabular}

Three models were built again on this data on the variables selected by the models which had given good accuracies measures

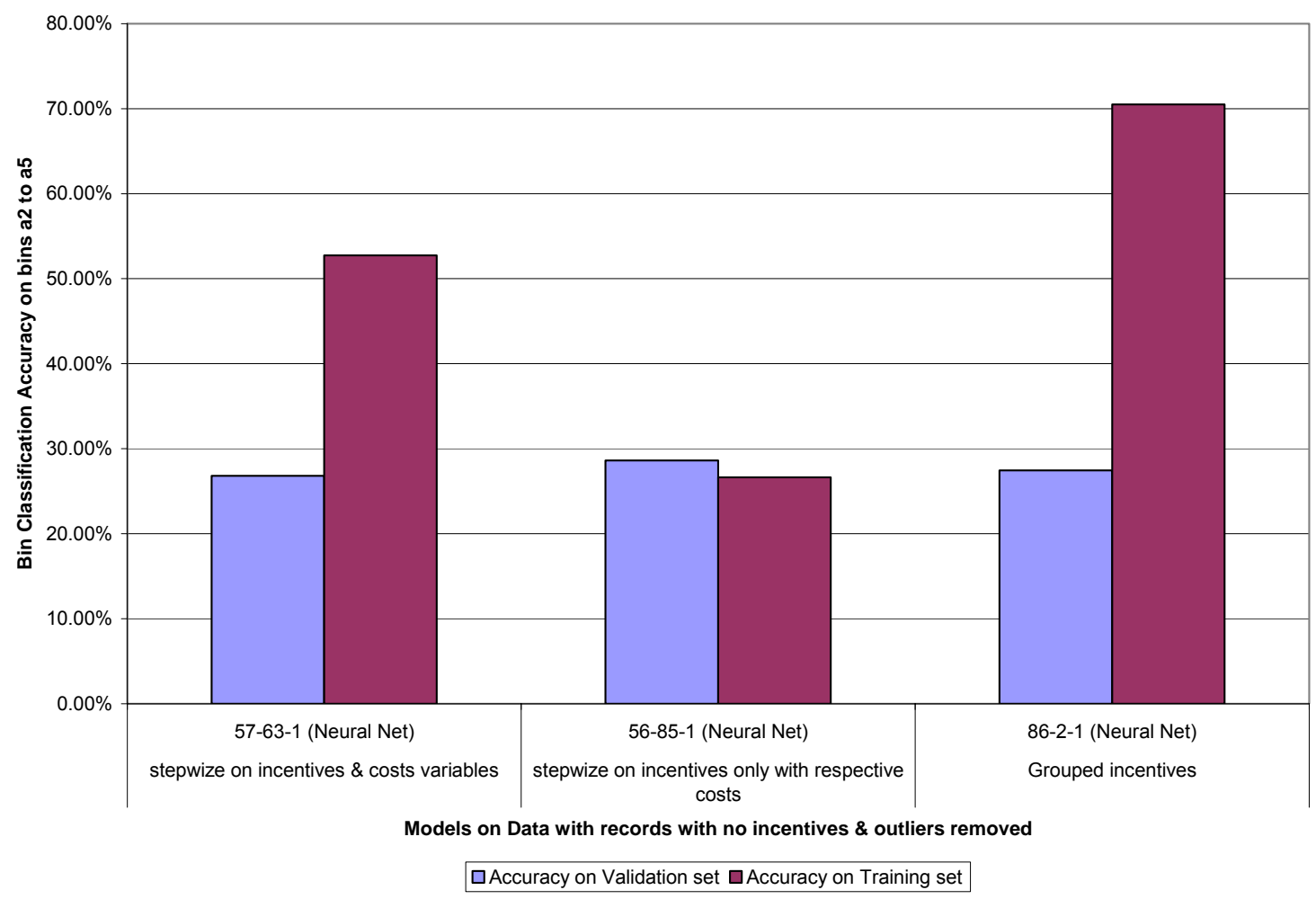

Figure 18: Bin Classification Accuracy on Moderate Range of change in VTR(a2 to a5) for validation

\& training set (Different models on data - records with no incentives \& outliers removed) 


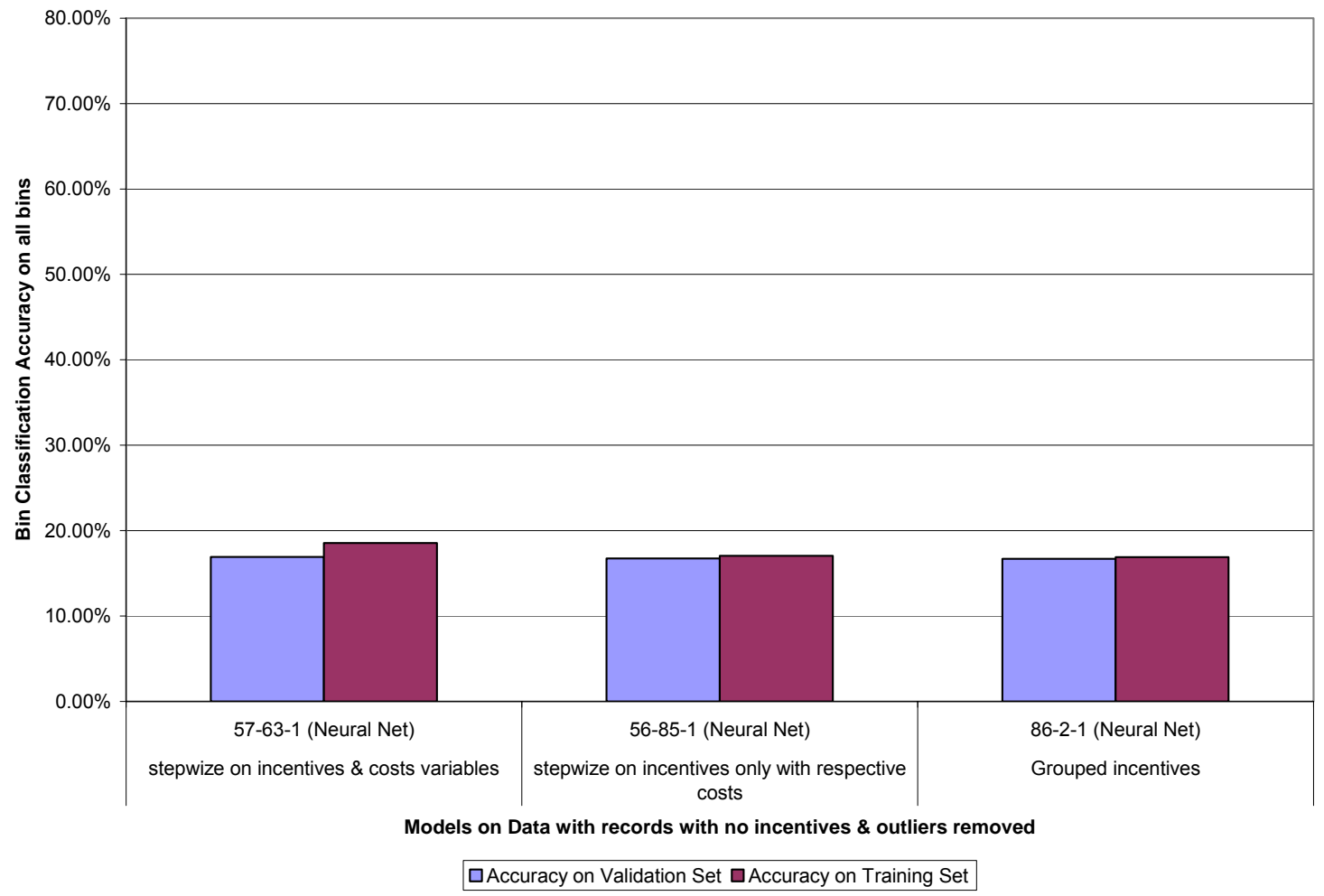

Figure 19: Bin Classification Accuracy on Full Range of change in VTR(all bins) for validation \& training set (Different models on data - records with no incentives \& outliers removed)

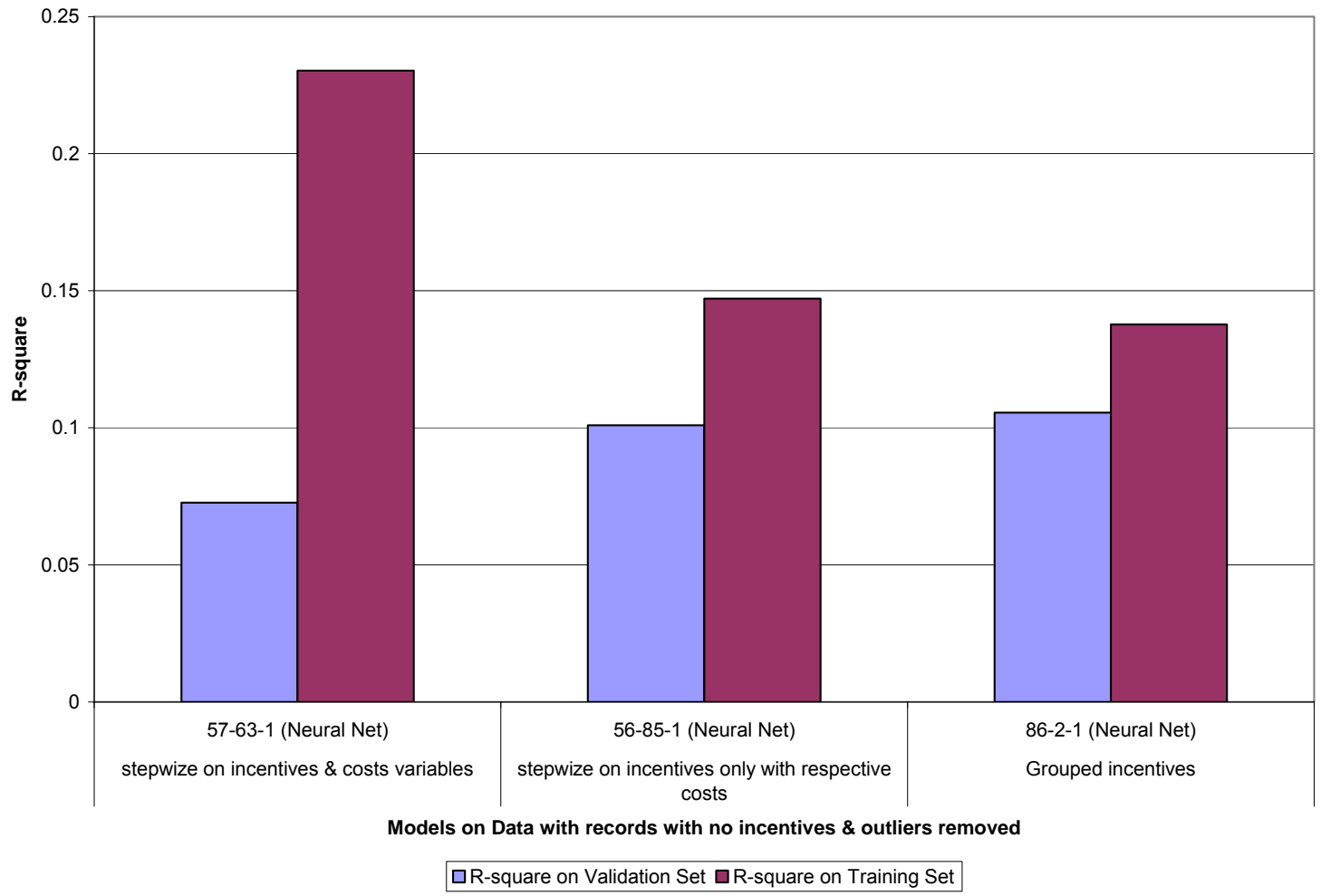

Figure 20: R-square for validation \& training set (Different models on data - records with no incentives \& outliers removed) 
Out of all of the models built without the outlier data, the model that got the best of three measures was the one built from stepwise regression on incentives with respective costs added. Figure 21 shows the scatter plot for this model on the validation set and it is apparent that this model was not a good predictor of change in VTR.

Actual Delta_VTR Vs. Predicted Delta_VTR

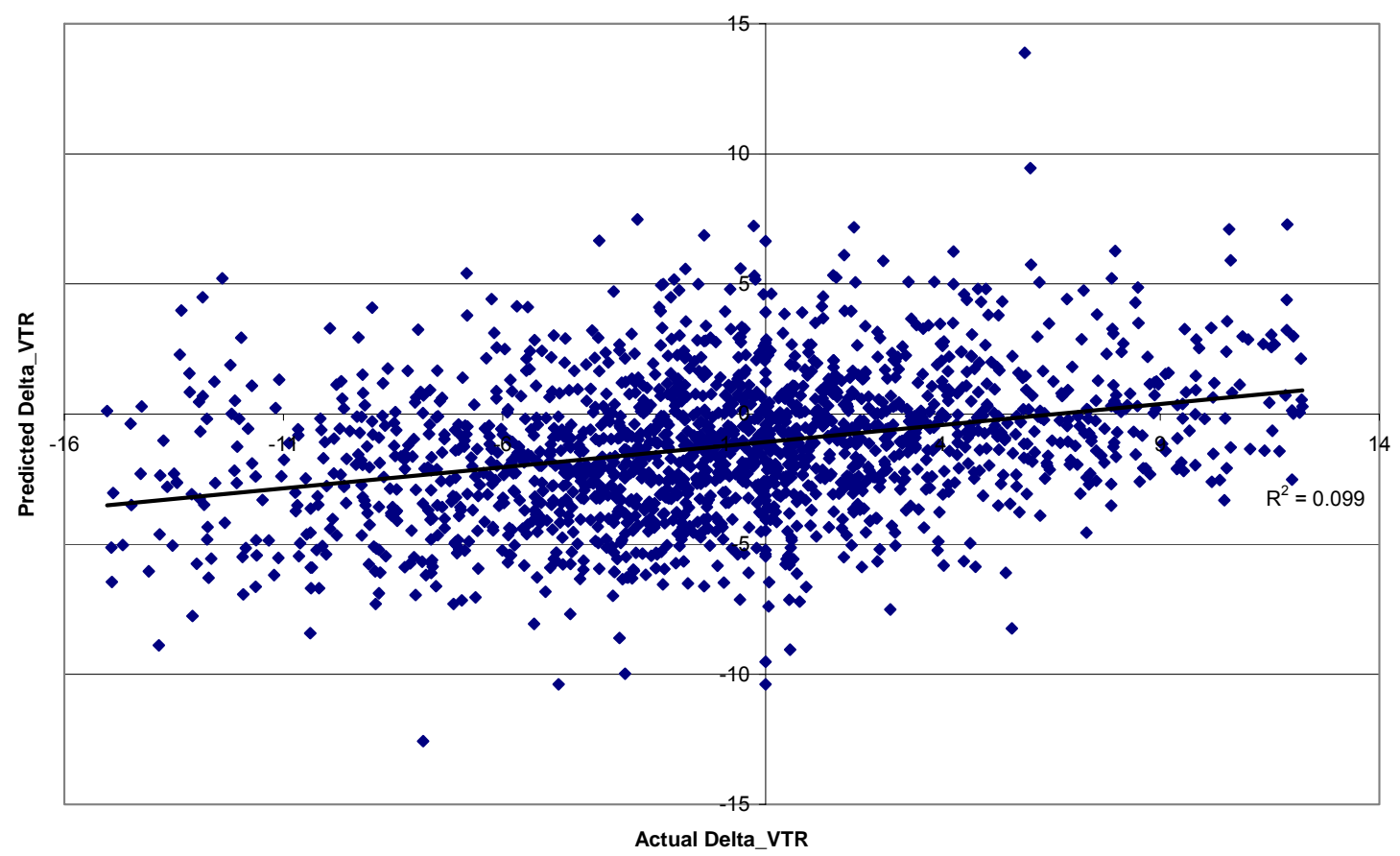

Figure 21: Scatter plot for model built on stepwise regression on incentives with respective costs added

Though the assumption about outliers might have been true, the removal of the outlier records further reduced the prediction power of these models. 


\section{Recommended Model}

In phase I and II of model building, the datasets contained some records which had no information about the incentives each worksite had implemented. This might have reduced the importance of incentives in predicting the change in VTR. In phase III, data was cleaned by deleting the records that did not have any information regarding implemented incentives. There was a considerable improvement in the accuracies in all the models as compared to the models built in the previous two phases.

In phase IV, although the outlier records were removed from the data, the total accuracies and the R-square values of the models went down. The two best candidate models were the ones built using the stepwise regression variables on incentives and costs and the other built on grouped incentives.

The reasons the model built on stepwise regression variables on incentives and costs was considered as candidate was (Table 10)

1. It has 'bin classification accuracy on moderate range of change in VTR' of $25.53 \%$

2. It has 'bin classification accuracy on full range of change in VTR' of $16.92 \%$ which is better than the random choice accuracy of $12.5 \%$

3. It has the best 'R-square' value of 0.1649

The reasons the model built on grouped incentives data was considered as candidate was (Table 10)

1. It has 'bin classification accuracy on moderate range of change in VTR' of $25.95 \%$

2. It has 'bin classification accuracy on full range of change in $\mathrm{VTR}^{\prime}$ of $16.92 \%$ which is better than the random choice accuracy of $12.5 \%$

3. It has a 'R-square' value of 0.1456

4. It is built on simple grouped incentive variable set

The variable set selected by the regression model was not complete as it did not cover all of the incentives, did contain some very detailed specific incentives like Taxi Guaranteed Return Trip dollar amount, etc. which the user of the model might not be able to provide. Thus due to the above reasons, the variable set would not have appealed to the transportation planners and engineers when compared to the simple grouped incentives variable set. So considering these facts, the recommended model is the one built on the grouped incentives with variables shown in the Table 12. 
Table 12: Variables for recommended model Los Angeles Model

\begin{tabular}{|c|c|}
\hline Variable & Description \\
\hline T_AVR1.3 & down town zone \\
\hline T_AVR1.5 & suburbs zone \\
\hline T_AVR1.75 & outside suburb zone \\
\hline VTR_CUTR & Vehicle trip rate \\
\hline CAR1 & Share of employees commuting by motorcycle \\
\hline Motorcycle & Share of employees commuting alone in car \\
\hline CAR2 & Share of employees commuting two together \\
\hline CAR3 & Share of employees commuting three together \\
\hline CAR4 & Share of employees commuting four together \\
\hline CAR5 & Share of employees commuting five together \\
\hline CAR6 & Share of employees commuting six together \\
\hline VAN_CUTR & Share of employees commuting in van \\
\hline BUS & Share of employees commuting in bus \\
\hline TRANSIT & Share of employees commuting using transit \\
\hline WALK & Share of employees commuting walking \\
\hline BIKE & Share of employees commuting using bike \\
\hline TELECOMMUTE & Share of employees telecommuting \\
\hline CWW336 & Share of $3 / 36 \mathrm{CWW}$ days off \\
\hline CWW440 & Share of $4 / 40 \mathrm{CWW}$ days off \\
\hline CWW980 & Share of $9 / 80 \mathrm{CWW}$ days off \\
\hline FACILITY_AMENITIES & $\begin{array}{l}\text { Passenger Loading Areas, Other Facility Improvements, Preferential Parking Areas, Bike } \\
\text { Racks and Bike Lockers, Shower and Lockers }\end{array}$ \\
\hline RIDE_HOME & $\begin{array}{l}\text { TMA/TMO Provided Guaranteed Return Trip, Company Vehicle Guaranteed Return Trip, } \\
\text { Emergencies Guaranteed Return Trip, Other Guaranteed Return Trip Program, Rental } \\
\text { Car Guaranteed Return Trip, Taxi Guaranteed Return Trip, Unscheduled Overtime } \\
\text { Guaranteed Return }\end{array}$ \\
\hline FLEXTIME & Flextime for Ride sharers (Work Shifts), Flextime for Ride sharers (Grace Period) \\
\hline MARKETING & $\begin{array}{l}\text { Commuter Information Center, Commuter Fairs (Marketing), Focus Groups (Marketing), } \\
\text { Posted Materials (Marketing), New Hire Orientation (Marketing), Other Marketing } \\
\text { Elements, Personal Communication (Marketing), Company Recognition (Marketing), } \\
\text { Special Interest Club (Biking, Walking), TMA/TMO Membership (Marketing), Written } \\
\text { Materials (Marketing), Zip Code Meetings (Marketing) }\end{array}$ \\
\hline RS_MATCH & Regional Commuter Management Agency, Employer-Based Rideshare Matching System \\
\hline FINANCIAL & $\begin{array}{l}\text { Transportation Allowances, On-Going Bike-to-Work Subsidies, On-Going Carpooling } \\
\text { Subsidies, Other Direct Financial Subsidies, On-Going Walk-to-Work Subsidies }\end{array}$ \\
\hline PARKMGT\$ & $\begin{array}{l}\text { Increased Parking Costs for Drive Alones, Other Parking Management Strategies, } \\
\text { Subsidized Parking for Ride sharers }\end{array}$ \\
\hline TELE_GRP & Work at Home (Telecommuting), Work at Satellite Center (Telecommuting) \\
\hline COMPRESSED & $\begin{array}{l}\text { 3/36 Compressed Work Week Schedule, 4/40 Compressed Work Week Schedule, 9/80 } \\
\text { Compressed Work Week Schedule, Other Compressed Work Week Schedule }\end{array}$ \\
\hline VANPOOL & Company Owned/Leased Vanpools \\
\hline ONSITE & $\begin{array}{l}\text { On-Site Childcare Service, Other On-Site Services, Cafeteria, ATM's, Postal, Fitness } \\
\text { Center, Transit Information or Pass Sales }\end{array}$ \\
\hline DIRECT_NONFINANCIAL & $\begin{array}{l}\text { Auto Services (Fuel, Oil, Tune-Up), Gift Certificates, Free Meals, Other Direct Non- } \\
\text { Financial Incentives, Catalogue Points, Additional Time Off with Pay, Drawings, Free } \\
\text { Meals, Certificates, etc. }\end{array}$ \\
\hline OTHER & Other Not Classified by Other Codes \\
\hline COMMTAX & $\begin{array}{l}\text { Introductory Transit Passes or Subsidies, Subsidized Vanpool Seats, On-Going Transit } \\
\text { Subsidies, On-Going Vanpooling Subsidies }\end{array}$ \\
\hline DeltaVTR & Change in VTR \\
\hline
\end{tabular}




\section{TUCSON MODELS}

\section{Phase I: Tucson Full Sample Data}

The Tucson dataset consisted of 1,121 total examples. Approximately ten percent of the dataset was left aside as validation set while the rest became training/testing set. Because the training/testing set contained just 1,009 examples, ten percent of the random data of was set aside as the testing set. The eight bin ranges and the number of examples in each bin for validation and training/testing set are shown in Table 13.

Table 13: Tucson Full Sample Data - No. of Records in bins

\begin{tabular}{|c|c|c|c|c|c|c|c|c|c|}
\hline \multirow{2}{*}{ Range } & \multirow{2}{*}{ \# of records } & \multicolumn{7}{|c|}{ Bin ranges over Change in Vehicle Trip Rate (Delta_VTR) } \\
\cline { 3 - 10 } & & $\mathbf{> - 7}$ & [- 7 to - 4.5) & [- - 4.5 to - 3) & [- 3 to -1.5) & [-1.5 to 0) & [0 to 1.5) & [1.5 to 3.5) & 3.5 >= \\
\hline Bin Number & & $\mathbf{a 1}$ & $\mathbf{a 2}$ & $\mathbf{a 3}$ & $\mathbf{a 4}$ & $\mathbf{a 5}$ & $\mathbf{a 6}$ & $\mathbf{A 7}$ & $\mathbf{a 8}$ \\
Validation & $\mathbf{1 1 2}$ & 4 & 6 & 6 & 13 & 19 & 25 & 22 & 17 \\
Training & $\mathbf{1 0 0 9}$ & 37 & 52 & 56 & 116 & 171 & 222 & 200 & 155 \\
\hline
\end{tabular}

Neural network models were built on this data with different variable selection parameters. All types of regression models were also built. Figures 22,23 , and 24, show the comparison of the different performance measures on this data.

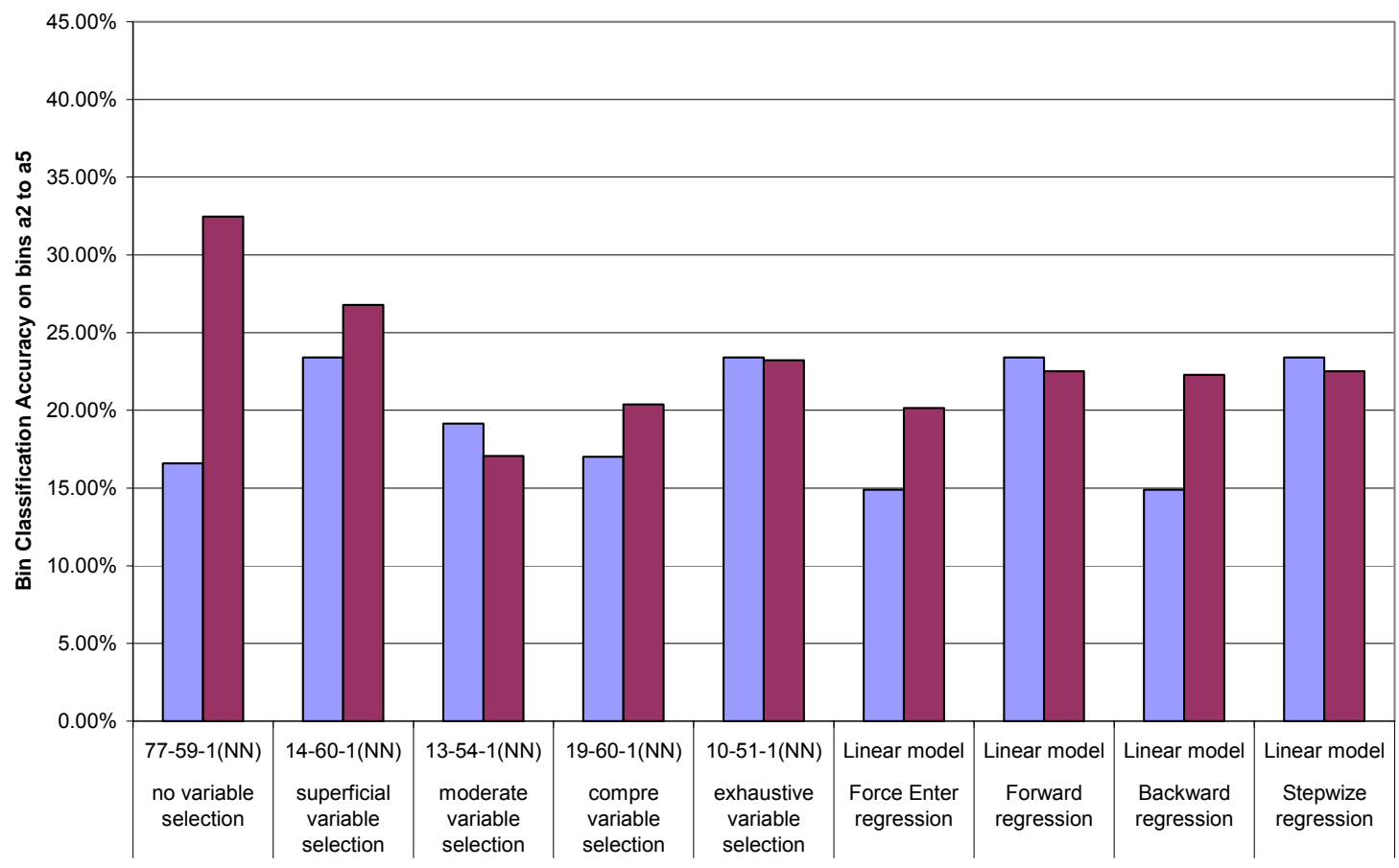

Models on Full Sample Data

$\square$ Accuracy on Validation set $\square$ Accuracy on Training set

Figure 22: Bin Classification Accuracy on Moderate Range of change in VTR(a2 to a5) for validation \& training set (Different models on Full sample data) 


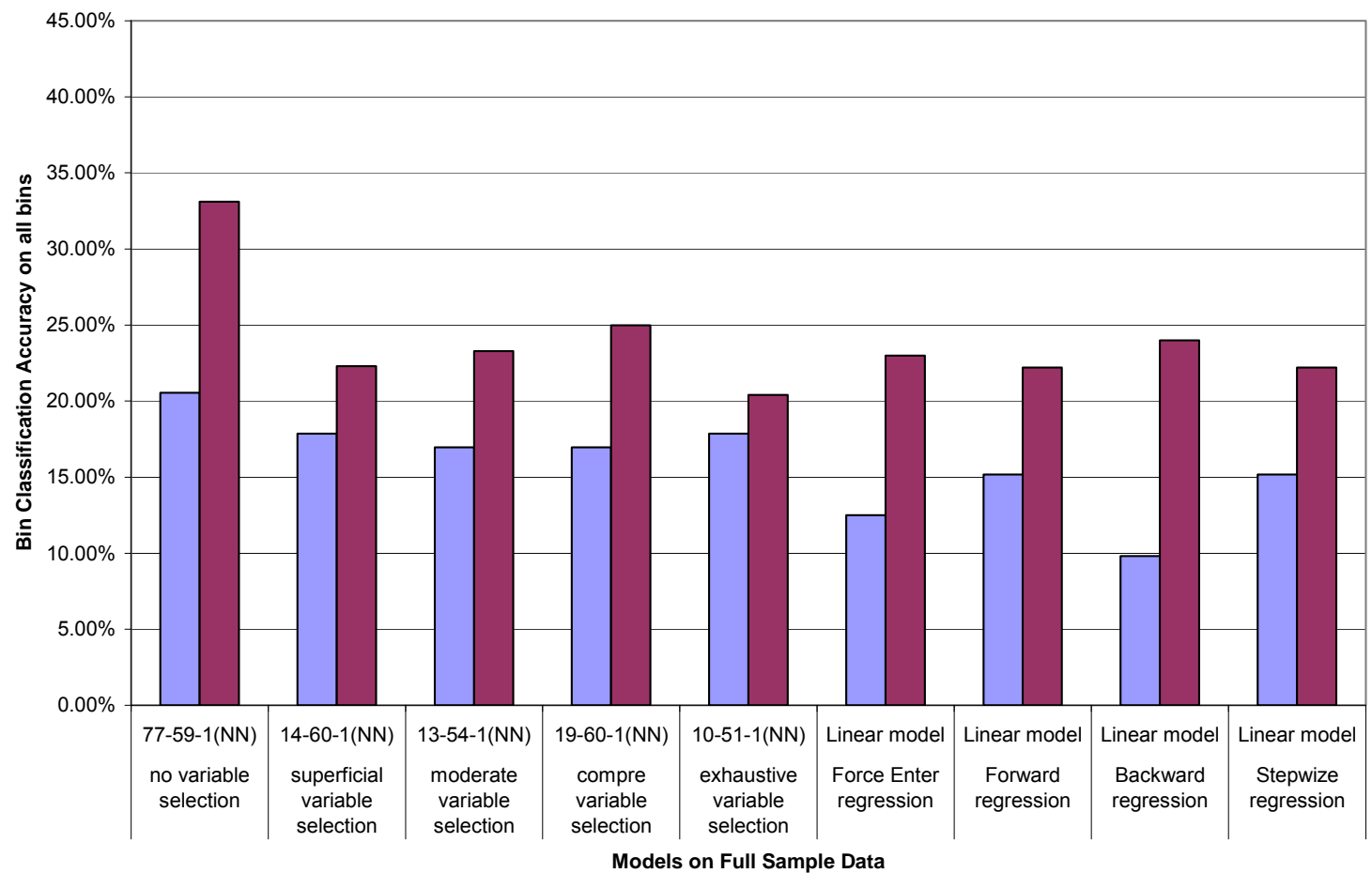

$\square$ Accuracy on Validation Set $\square$ Accuracy on Training Set

Figure 23: Bin Classification Accuracy (all bins) (Full sample data)

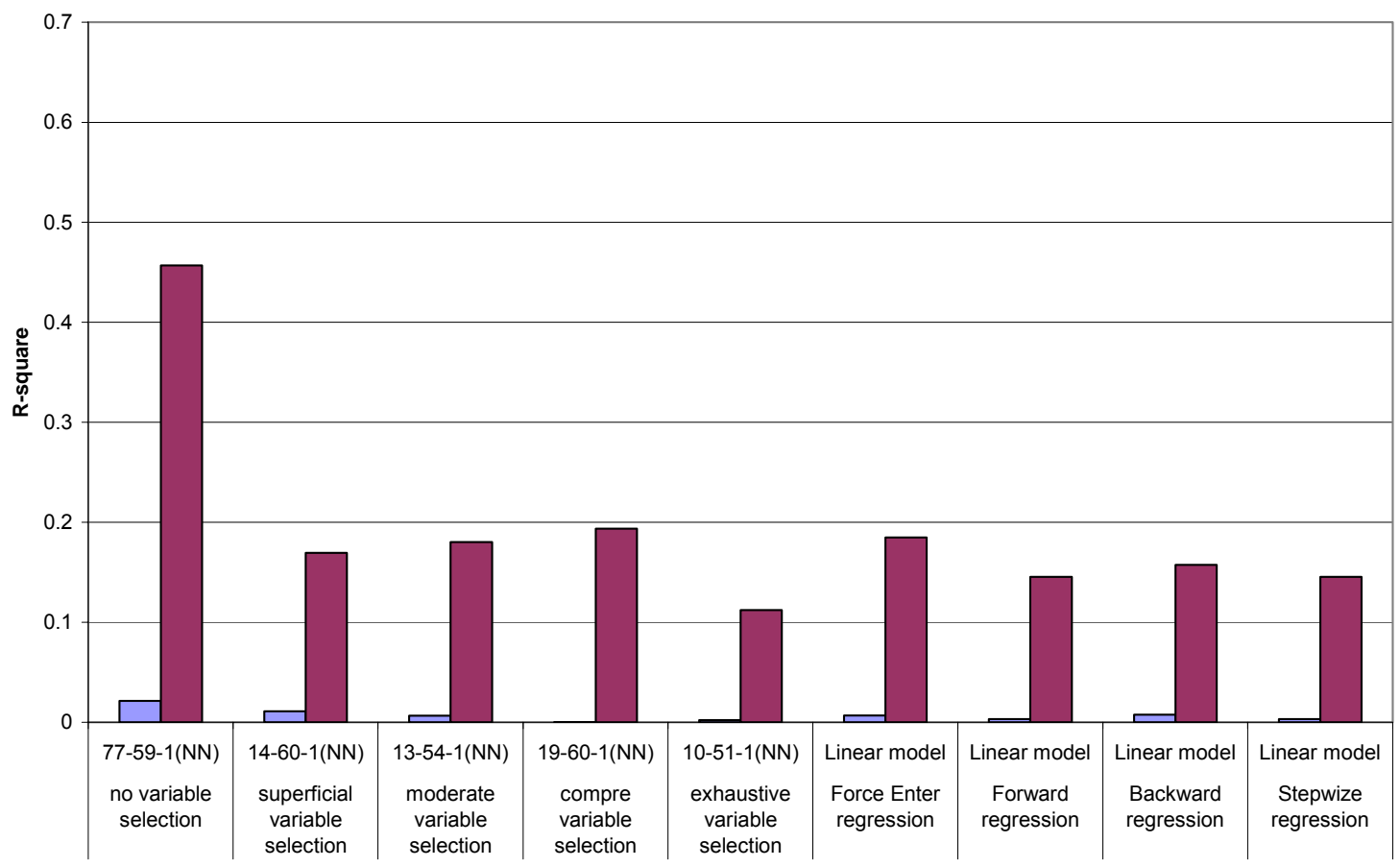

Models on Full Sample Data

$\square R$-square on Validation Set $\square$ R-square on Training Set

Figure 24: R-square for validation \& training set (Different models on Full sample data) 
The variables selected by these models are shown below in Table 14.

Table 14: Variables selected by different models on Full Sample data

\begin{tabular}{|c|c|c|c|c|c|c|c|c|c|}
\hline \multirow[b]{2}{*}{ variables } & \multicolumn{5}{|c|}{ Predicts variable selection } & \multicolumn{4}{|c|}{ Regression Approaches } \\
\hline & no var & $\begin{array}{c}\text { Superfic } \\
\text { ial }\end{array}$ & $\begin{array}{c}\text { modera } \\
\text { te }\end{array}$ & $\begin{array}{l}\text { Compre } \\
\text { hensive }\end{array}$ & $\begin{array}{c}\text { Exhaust } \\
\text { ive }\end{array}$ & $\begin{array}{c}\text { forced } \\
\text { enter }\end{array}$ & $\begin{array}{c}\text { For } \\
\text { ward }\end{array}$ & $\begin{array}{c}\text { Backw } \\
\text { ard }\end{array}$ & $\begin{array}{c}\text { Stepwi } \\
\text { se }\end{array}$ \\
\hline Alone share & $\sqrt{ }$ & & $\sqrt{ }$ & $\sqrt{ }$ & & & & & \\
\hline Bus share & $\sqrt{ }$ & $\sqrt{ }$ & $\sqrt{ }$ & $\sqrt{ }$ & $\sqrt{ }$ & $\sqrt{ }$ & & $\sqrt{ }$ & \\
\hline Carpool + Vanpool share & $\sqrt{ }$ & $\sqrt{ }$ & & & $\sqrt{ }$ & $\sqrt{ }$ & & $\sqrt{ }$ & \\
\hline walk share & $\sqrt{ }$ & $\sqrt{ }$ & $\sqrt{ }$ & $\sqrt{ }$ & & $\sqrt{ }$ & & $\sqrt{ }$ & \\
\hline Motorcycle share & $\sqrt{ }$ & $\sqrt{ }$ & $\sqrt{ }$ & $\sqrt{ }$ & & $\sqrt{ }$ & $\sqrt{ }$ & $\sqrt{ }$ & $\sqrt{ }$ \\
\hline Bicycle share & $\sqrt{ }$ & $\sqrt{ }$ & $\sqrt{ }$ & & $\sqrt{ }$ & $\sqrt{ }$ & $\sqrt{ }$ & $\sqrt{ }$ & $\sqrt{ }$ \\
\hline Average Miles traveled & $\sqrt{ }$ & & $\sqrt{ }$ & $\sqrt{ }$ & $\sqrt{ }$ & $\sqrt{ }$ & & & \\
\hline Average minutes traveled & $\sqrt{ }$ & & & $\sqrt{ }$ & $\sqrt{ }$ & $\sqrt{ }$ & & & \\
\hline $3 / 36$ compressed work week share & $\sqrt{ }$ & & $\sqrt{ }$ & $\sqrt{ }$ & & $\sqrt{ }$ & & & \\
\hline $4 / 40$ compressed work week share & $\sqrt{ }$ & $\sqrt{ }$ & $\sqrt{ }$ & $\sqrt{ }$ & & $\sqrt{ }$ & $\sqrt{ }$ & $\sqrt{ }$ & $\sqrt{ }$ \\
\hline $8 / 80$ compressed work week share & $\sqrt{ }$ & $\sqrt{ }$ & $\sqrt{ }$ & & & $\sqrt{ }$ & & & \\
\hline Number of Employees & $\sqrt{ }$ & $\sqrt{ }$ & & & & $\sqrt{ }$ & & & \\
\hline Adjusted Work Hours & & & & & & & & & \\
\hline Alternative Fuel Vehicles & $\sqrt{ }$ & & & & & $\sqrt{ }$ & & & \\
\hline Alternate Mode Campaign & $\sqrt{ }$ & & & $\sqrt{ }$ & & $\sqrt{ }$ & & & \\
\hline Alternate Mode Information & $\sqrt{ }$ & & & & & $\sqrt{ }$ & & & \\
\hline Bicycle Campaign & $\sqrt{ }$ & & & $\sqrt{ }$ & & $\sqrt{ }$ & & & \\
\hline Bicycle Racks & $\sqrt{ }$ & & & & & $\sqrt{ }$ & & & \\
\hline Bus Pass Sales on Site & & & & & & $\sqrt{ }$ & & & \\
\hline Bus Subsidy & & & & & & $\sqrt{ }$ & & & \\
\hline Busing Vehicle & $\sqrt{ }$ & & & & & $\sqrt{ }$ & & & \\
\hline Carpool Campaign & $\sqrt{ }$ & & & & & $\sqrt{ }$ & & & \\
\hline Carpool Subsidy & $\sqrt{ }$ & & & & & $\sqrt{ }$ & & & \\
\hline Carpooling Vehicle & $\sqrt{ }$ & & & & & $\sqrt{ }$ & & & \\
\hline Compressed Work Week & $\sqrt{ }$ & & & & & $\sqrt{ }$ & & & \\
\hline Coordination with Transit Provider & $\sqrt{ }$ & & & & & $\sqrt{ }$ & & & \\
\hline Covered Parking & $\sqrt{ }$ & $\sqrt{ }$ & & & & $\sqrt{ }$ & & & \\
\hline Daycare Facilities on Site & $\sqrt{ }$ & & & & & $\sqrt{ }$ & & & \\
\hline Dissemination of Air Quality Information & $\sqrt{ }$ & & & & $\sqrt{ }$ & $\sqrt{ }$ & & & \\
\hline Drawing for Prizes & $\sqrt{ }$ & $\sqrt{ }$ & $\sqrt{ }$ & $\sqrt{ }$ & & $\sqrt{ }$ & & & \\
\hline Employee Shifts Between Sites & $\sqrt{ }$ & & & & $\sqrt{ }$ & $\sqrt{ }$ & & $\sqrt{ }$ & \\
\hline Fee for Parking & $\sqrt{ }$ & & & & & $\sqrt{ }$ & & & \\
\hline Field Worker & $\sqrt{ }$ & & & & & $\sqrt{ }$ & & & \\
\hline Guaranteed Ride Home Program & $\sqrt{ }$ & & & & $\sqrt{ }$ & $\sqrt{ }$ & & & \\
\hline Incentive Programs & $\sqrt{ }$ & & & & & $\sqrt{ }$ & & & \\
\hline Incentives for Employees to Live Close & $\sqrt{ }$ & & & & & $\sqrt{ }$ & & & \\
\hline Information Center & $\sqrt{ }$ & & & & & $\sqrt{ }$ & & & \\
\hline Map Board & $\sqrt{ }$ & & & $\sqrt{ }$ & & $\sqrt{ }$ & & & \\
\hline Matching Service & & & & & & & & & \\
\hline New Employee Information & $\sqrt{ }$ & & & & & $\sqrt{ }$ & & & \\
\hline Newsletter Articles & $\sqrt{ }$ & & $\sqrt{ }$ & & & $\sqrt{ }$ & & & \\
\hline Post Air Quality Information & $\sqrt{ }$ & & & $\sqrt{ }$ & & $\sqrt{ }$ & & & \\
\hline Preferred Parking & & & & & & & & & \\
\hline Rebate not to Use Parking & $\sqrt{ }$ & & & & & $\sqrt{ }$ & & & \\
\hline Rideshare Committee & $\sqrt{ }$ & & & $\sqrt{ }$ & & $\sqrt{ }$ & & & \\
\hline Showers/Lockers & $\sqrt{ }$ & & & & & $\sqrt{ }$ & & & \\
\hline Shuttle Vehicle & $\sqrt{ }$ & & & $\sqrt{ }$ & & $\sqrt{ }$ & & $\sqrt{ }$ & \\
\hline Speakers & $\sqrt{ }$ & & & & & $\sqrt{ }$ & & $\sqrt{ }$ & \\
\hline Staging Area & $\sqrt{ }$ & & $\sqrt{ }$ & & & $\sqrt{ }$ & & & \\
\hline Transportation Fair & & & & & & $\sqrt{ }$ & & & \\
\hline Vanpooling Vehicle & & & & & & $\sqrt{ }$ & & & \\
\hline Vanpool Subsidy & $\sqrt{ }$ & $\sqrt{ }$ & & & & $\sqrt{ }$ & $\sqrt{ }$ & $\sqrt{ }$ & $\sqrt{ }$ \\
\hline Vanpooling Vehicle & $\sqrt{ }$ & & & & & $\sqrt{ }$ & & & \\
\hline Walking Campaign & $\sqrt{ }$ & & & & & $\sqrt{ }$ & & & \\
\hline Work at Home & $\sqrt{ }$ & & & & & $\sqrt{ }$ & & & \\
\hline VTR & $\sqrt{ }$ & & $\sqrt{ }$ & & & $\sqrt{ }$ & $\sqrt{ }$ & $\sqrt{ }$ & $\sqrt{ }$ \\
\hline
\end{tabular}

It can be seen from the performance measures charts, that neural network models built using superficial and exhaustive variable selection and linear models built using forward and stepwise regression were able to get the best 'bin classification accuracy on moderate range of change in VTR' (23.40 percent). But, all of these models had very poor R-square values. It can be 
seen from the variable selection table, that the variables selected by all of these models contain very few incentive plans, thus defeating the purpose of the models to predict change in VTR using many incentives. All the neural network and regression models built using variable selection selected very few incentives variables and were considered as unsuitable models.

The neural network model built with no variable selection got the best 'bin classification accuracy on full range of change in VTR' (20.54 percent) and best $\mathrm{R}$-square value $(0.022)$, and could be considered as a better model. The detailed bin accuracies are shown in Table 15 and the scatter plot in Figure 25.

Table 15: Detailed accuracies for neural net model with no variable selection

\begin{tabular}{|c|c|c|c|c|c|c|c|c|c|c|c|}
\hline \multirow[b]{2}{*}{ range } & \multirow{2}{*}{$\begin{array}{c}\text { \# of } \\
\text { records }\end{array}$} & \multicolumn{8}{|c|}{ Bin ranges over Change in Vehicle Trip Rate (Delta_VTR) } & \multirow{6}{*}{$\begin{array}{c}\text { Weighted } \\
\text { Avg on } \\
\text { a2 to a5 }\end{array}$} & \multirow{6}{*}{ R-square } \\
\hline & & $>-7$ & $\begin{array}{c}{[-7 \text { to }-} \\
4.5)\end{array}$ & $\begin{array}{c}{[-4.5 \text { to }} \\
-3)\end{array}$ & $\begin{array}{c}{[-3 \text { to }-} \\
1.5)\end{array}$ & $\begin{array}{c}{[-1.5 \text { to }} \\
0)\end{array}$ & $\begin{array}{l}\text { [0 to } \\
1.5)\end{array}$ & $\begin{array}{c}{[1.5 \text { to }} \\
3.5)\end{array}$ & $3.5>=$ & & \\
\hline Bin Number & & a1 & a2 & a3 & a4 & a5 & a6 & a7 & a8 & & \\
\hline Validation & 112 & 4 & 6 & 6 & 13 & 19 & 25 & 22 & 17 & & \\
\hline \multirow[t]{2}{*}{ Training } & 1009 & 37 & 52 & 56 & 116 & 171 & 222 & 200 & 155 & & \\
\hline & & & & & & & & & & & \\
\hline Exact Validation & $20.54 \%$ & $0.00 \%$ & $0.00 \%$ & $20.00 \%$ & $23.53 \%$ & $11.76 \%$ & $14.29 \%$ & $36.84 \%$ & $25.00 \%$ & $16.60 \%$ & 0.022 \\
\hline Exact Training & $33.10 \%$ & $0.00 \%$ & $24.56 \%$ & $29.85 \%$ & $28.23 \%$ & $39.08 \%$ & $27.67 \%$ & $31.49 \%$ & $50.61 \%$ & $32.46 \%$ & 0.457 \\
\hline One-off Validation & $43.75 \%$ & $0.00 \%$ & $0.00 \%$ & $60.00 \%$ & $41.18 \%$ & $41.18 \%$ & $52.38 \%$ & $47.37 \%$ & $45.00 \%$ & $41.28 \%$ & \\
\hline One-Off Training & $70.86 \%$ & $38.89 \%$ & $57.89 \%$ & $49.25 \%$ & $65.32 \%$ & $79.31 \%$ & $76.21 \%$ & $71.82 \%$ & $78.66 \%$ & & \\
\hline
\end{tabular}

Actual Delta_VTR vs. Predicted Delta VTR

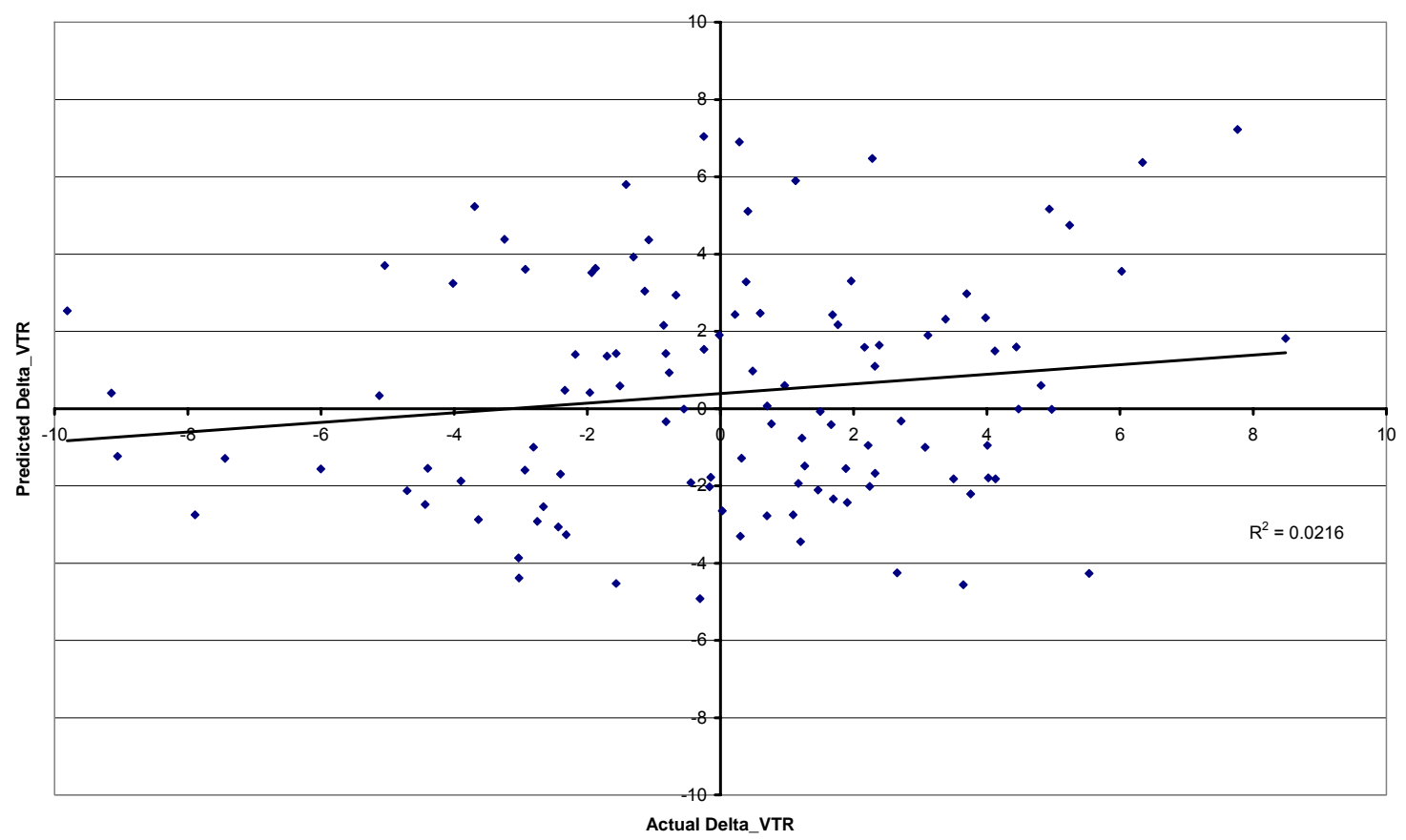

Figure 25: Scatter plot for neural net model with no variable selection 
To reduce to complexity of the models, all the individual incentives were removed and then replaced with the grouped incentives and simple neural network with no variable selection and forced enter linear regression models were built on the data just containing the grouped incentives and worksite characteristics like mode-splits. The variables for the Tucson grouped incentives model are shown in Table 16.

Table 16: Variables with grouped incentives

\begin{tabular}{|l|l|}
\hline \multicolumn{1}{|c|}{ Variable } & \multicolumn{1}{c|}{ Description } \\
\hline AloneShare & Alone share \\
BusShare & Bus share \\
CVpoolShare & Carpool + Vanpool share \\
WalkShare & walk share \\
McycleShare & Motorcycle share \\
BcycleShare & Bicycle share \\
HiMiles & Average Miles traveled \\
HiMinute & Average minutes traveled \\
CWW336 & 3/36 compressed work week share \\
CWW440 & 4/40 compressed work week share \\
CWW980 & 8/80 compressed work week share \\
NoEmp & No. of employees \\
FACILITY_AMENITIES & facilities \& amenities (grouped incentives) \\
GRH & Guaranteed ride home programs (grouped incentives) \\
FLEX & flexible timing (grouped incentives) \\
Mrkt & Marketing programs(grouped incentives) \\
RS_MATCH & Ride share matching programs(grouped incentives) \\
FINANCIAL & financial incentives(grouped incentives) \\
PARKMGT & Parking management (grouped incentives) \\
TELE & Telecommute program (grouped incentives) \\
CWW & Compressed work week program(grouped incentives) \\
VANPOOL & Vanpool vehicles (grouped incentives) \\
onsite & onsite incentives (grouped incentives) \\
direct_nonfinan & Non financial incentives (grouped incentives) \\
Commtax & commuter tax benefit incentives (grouped incentives) \\
VTR & Vehicle trip rate \\
\hline
\end{tabular}

Figures 26, 27, and 28 show the comparison of the different performance measures on this grouped incentive data. 


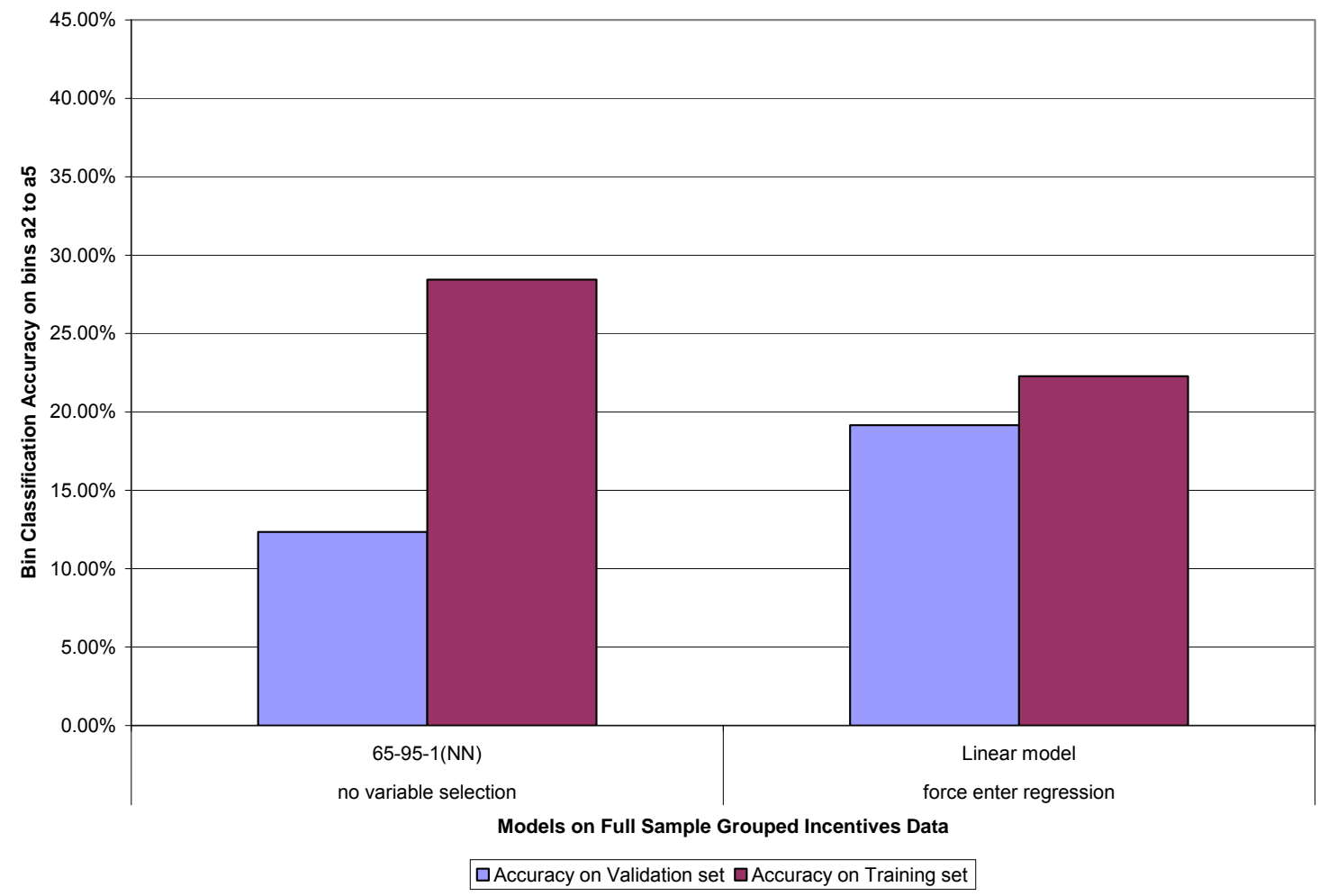

Figure 26: Bin Classification Accuracy on Moderate Range of change in VTR (a2 to a5) (Different models on Full sample Grouped Incentive data)

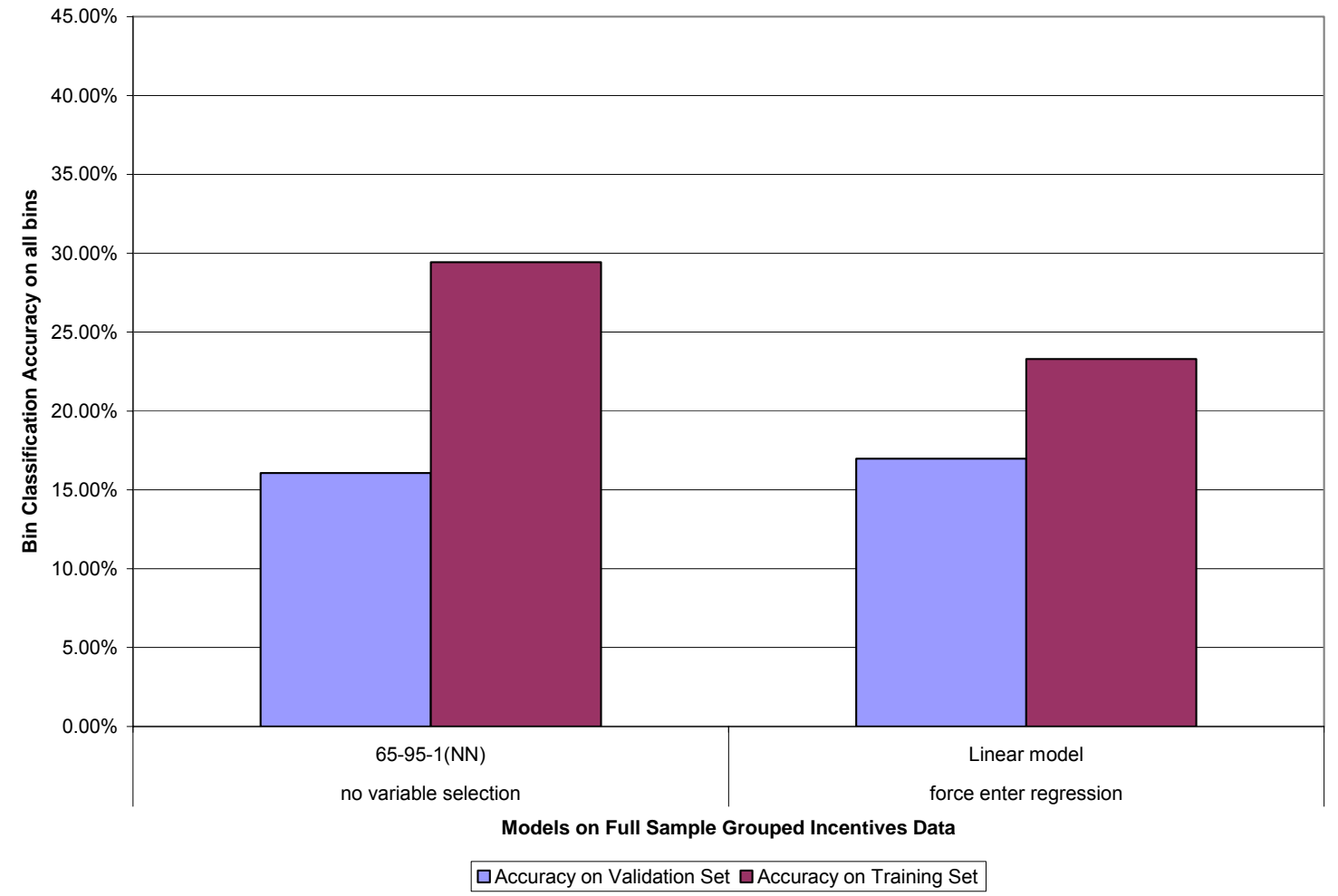

Figure 27: Bin Classification Accuracy on Full Range of change in VTR (all bins) (Different models on Full sample Grouped Incentive data) 


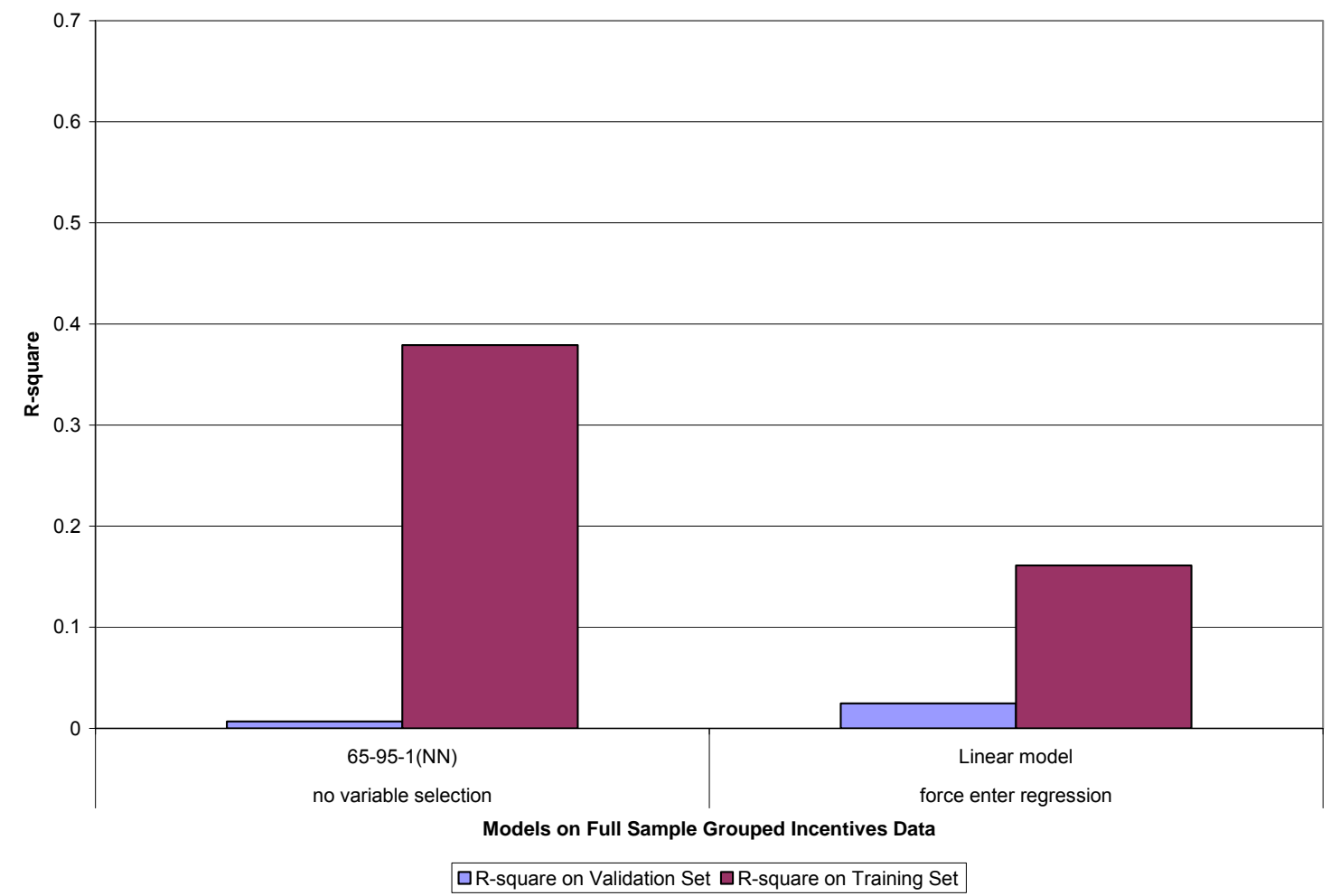

Figure 28: R-square for validation \& training set (Different models on Full sample Grouped Incentive data)

It can be seen from Figures 26 and 27 that the neural network gets much better accuracy on the training set than the regression models but lower accuracy on the validation set. A possible explanation for this anomalous behavior is that the neural network model might be over-fitting the training data, thereby making it useless for the validation set. In these two models, the forced enter regression model was clearly the best. The detailed accuracies of the bins for this model are shown in Table 17.

Table 17: Detailed accuracies for forced enter regression model

\begin{tabular}{|c|c|c|c|c|c|c|c|c|c|c|c|}
\hline \multirow[b]{2}{*}{ range } & \multirow{2}{*}{$\begin{array}{c}\text { \# of } \\
\text { records }\end{array}$} & \multicolumn{8}{|c|}{ Bin ranges over Change in Vehicle Trip Rate (Delta_VTR) } & \multirow{6}{*}{$\begin{array}{c}\text { Weighted } \\
\text { Avg on } \\
\text { a2 to a5 }\end{array}$} & \multirow{6}{*}{ R-square } \\
\hline & & $>-7$ & $\begin{array}{c}{[-7 \text { to }-} \\
4.5)\end{array}$ & $\begin{array}{c}{\left[\begin{array}{c}-4.5 \\
-3)\end{array}\right.} \\
\text { to }\end{array}$ & $\begin{array}{c}{[-3 \text { to }-} \\
1.5)\end{array}$ & $\begin{array}{c}{[-1.5 \text { to }} \\
0)\end{array}$ & $\begin{array}{c}\text { [0 to } \\
1.5)\end{array}$ & $\begin{array}{c}{[1.5 \text { to }} \\
3.5)\end{array}$ & $3.5>=$ & & \\
\hline Bin Number & & a1 & a2 & a3 & a4 & a5 & a6 & a7 & a8 & & \\
\hline Validation & 112 & 4 & 6 & 6 & 13 & 19 & 25 & 22 & 17 & & \\
\hline Training & 1009 & 37 & 52 & 56 & 116 & 171 & 222 & 200 & 155 & & \\
\hline & & & & & & & & & & & \\
\hline Exact Validation & $16.96 \%$ & $0.00 \%$ & $0.00 \%$ & $0.00 \%$ & $17.65 \%$ & $35.29 \%$ & $33.33 \%$ & $10.53 \%$ & $5.00 \%$ & $19.15 \%$ & 0.0248 \\
\hline Exact Training & $23.29 \%$ & $0.00 \%$ & $0.00 \%$ & $0.00 \%$ & $9.68 \%$ & $47.13 \%$ & $36.41 \%$ & $16.02 \%$ & $22.56 \%$ & $22.27 \%$ & 0.161 \\
\hline One-off Validation & $55.36 \%$ & $0.00 \%$ & $0.00 \%$ & $0.00 \%$ & $52.94 \%$ & $82.35 \%$ & $76.19 \%$ & $84.21 \%$ & $35.00 \%$ & $48.94 \%$ & \\
\hline One-Off Training & $59.96 \%$ & $0.00 \%$ & $0.00 \%$ & $19.40 \%$ & $66.13 \%$ & $87.36 \%$ & $88.35 \%$ & $56.35 \%$ & $45.12 \%$ & & \\
\hline
\end{tabular}


From Table 17 it can be seen that the linear regression model has zero accuracy in bins $a 1, a 2$ and a3. The scatter plot for this model is shown in Figure 29. Ideally, the best model would have an equal distribution of accuracy throughout the bin structure and be able to fit the full range of real delta VTR data, as well as predicting positive and negative values correctly.

Actual Delta_VTR vs. Predicted Delta_VTR

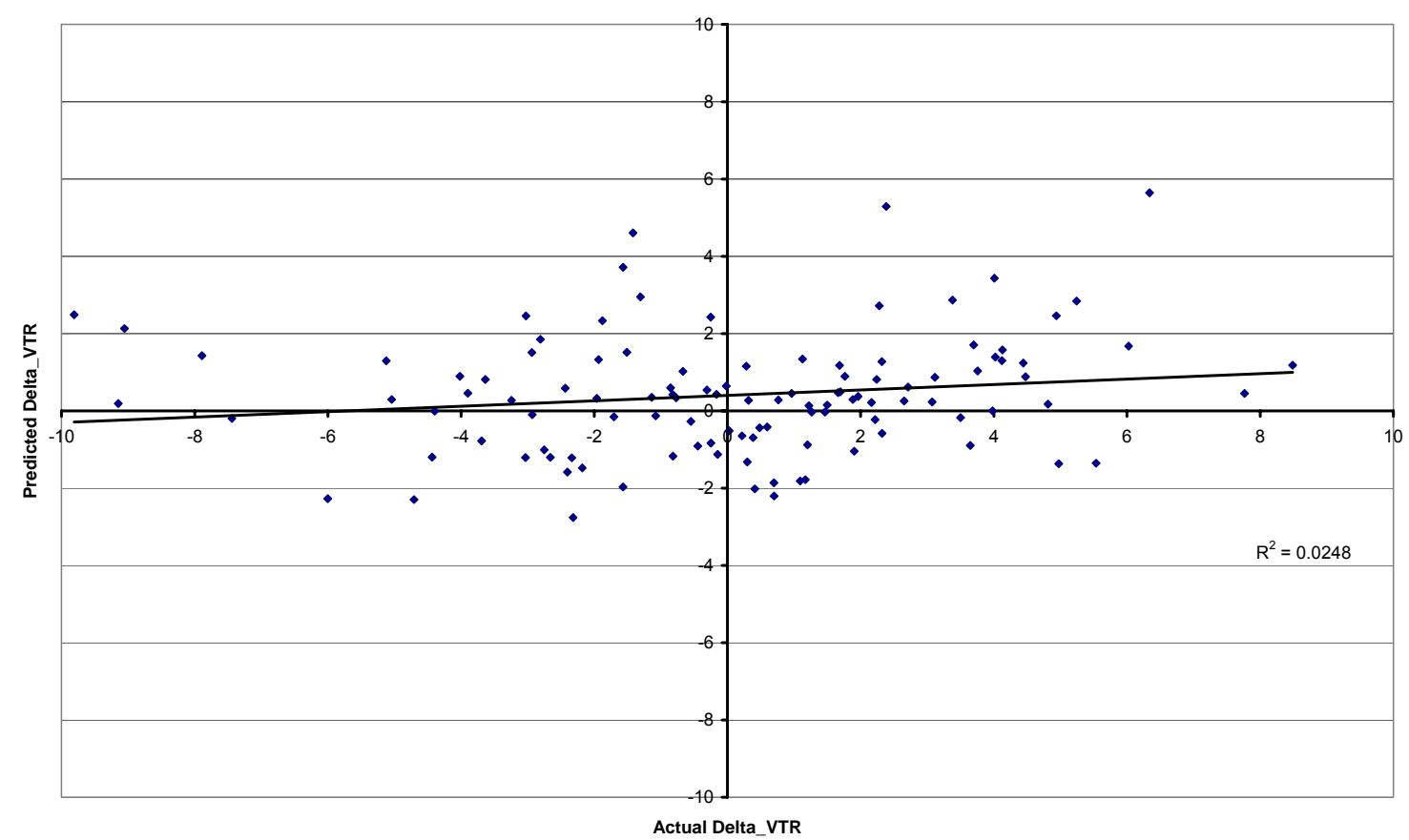

Figure 29: Scatter plot for forced enter linear regression model 


\section{The regression coefficients for this model are shown in Table 18.}

Table 18: Variables and the associated forced enter regression coefficients

\begin{tabular}{|l|r|r|}
\hline \multicolumn{1}{|c|}{ Variables } & coefficient & t - value \\
\hline (Constant) & -27.605 & -1.749 \\
Bus share & 36.169 & 2.231 \\
Carpool + Vanpool share & 24.729 & 2.838 \\
walk share & 40.915 & 2.449 \\
Motorcycle share & 77.932 & 4.297 \\
Bicycle share & 96.179 & 4.393 \\
Average Miles traveled & 0.010 & 1.100 \\
Average minutes traveled & -0.023 & -0.287 \\
3/36 compressed work week share & -0.004 & -0.087 \\
4/40 compressed work week share & 0.147 & 0.661 \\
8/80 compressed work week share & 0.004 & 1.184 \\
No. of employees & 0.000 & 0.276 \\
facilities \& amenities(grouped incentives) & 0.804 & 1.361 \\
Guaranteed ride home programs(grouped incentives) & 0.351 & 1.129 \\
flexible timing (grouped incentives) & -0.484 & -1.620 \\
Ride share matching programs(grouped incentives) & -1.456 & -0.342 \\
financial incentives(grouped incentives) & -0.377 & -0.680 \\
Parking management (grouped incentives) & -0.692 & -0.885 \\
Telecommuting (grouped incentives) & 0.465 & 1.453 \\
Compressed work week(grouped incentives) & 0.363 & 1.128 \\
Vanpool vehicles(grouped incentives) & -0.673 & -1.364 \\
onsite incentives(grouped incentives) & 0.116 & 0.339 \\
Non financial (grouped incentives) & -0.028 & -0.057 \\
commuter tax benefit incentives(grouped incentives) & -0.385 & -0.933 \\
Vehicle trip rate & 0.246 & 1.595 \\
\hline
\end{tabular}




\section{Phase II: Tucson Over-Sampled Data}

To get better accuracies on the moderate range bins a2 to a5, the examples in these bins were over-sampled. Table 19 shows the changes in the number of examples in training set due to over-sampling.

Table 19: Tucson Over-Sampled Data - No. of Records in bins

\begin{tabular}{|c|c|c|c|c|c|c|c|c|c|}
\hline \multirow{2}{*}{ Range } & \multirow{2}{*}{ \# of records } & \multicolumn{8}{|c|}{ Bin ranges over Change in Vehicle Trip Rate (Delta_VTR) } \\
\hline & & $>-7$ & [- 7 to -4.5$)$ & {$[-4.5$ to -3$)$} & [- 3 to -1.5$)$ & {$[-1.5$ to 0$)$} & (0 to 1.5) & [1.5 to 3.5 ) & $3.5>=$ \\
\hline Bin Number & & a1 & a2 & a3 & a4 & a5 & a6 & a7 & a8 \\
\hline Validation & 112 & 4 & 6 & 6 & 13 & 19 & 25 & 22 & 17 \\
\hline Training & 1612 & 142 & 221 & 215 & 248 & 226 & 215 & 181 & 164 \\
\hline
\end{tabular}

Neural network and regression models with different variable selection were built on this data. Figures 30,31 , and 32 show the comparison of the different performance measures on this data.

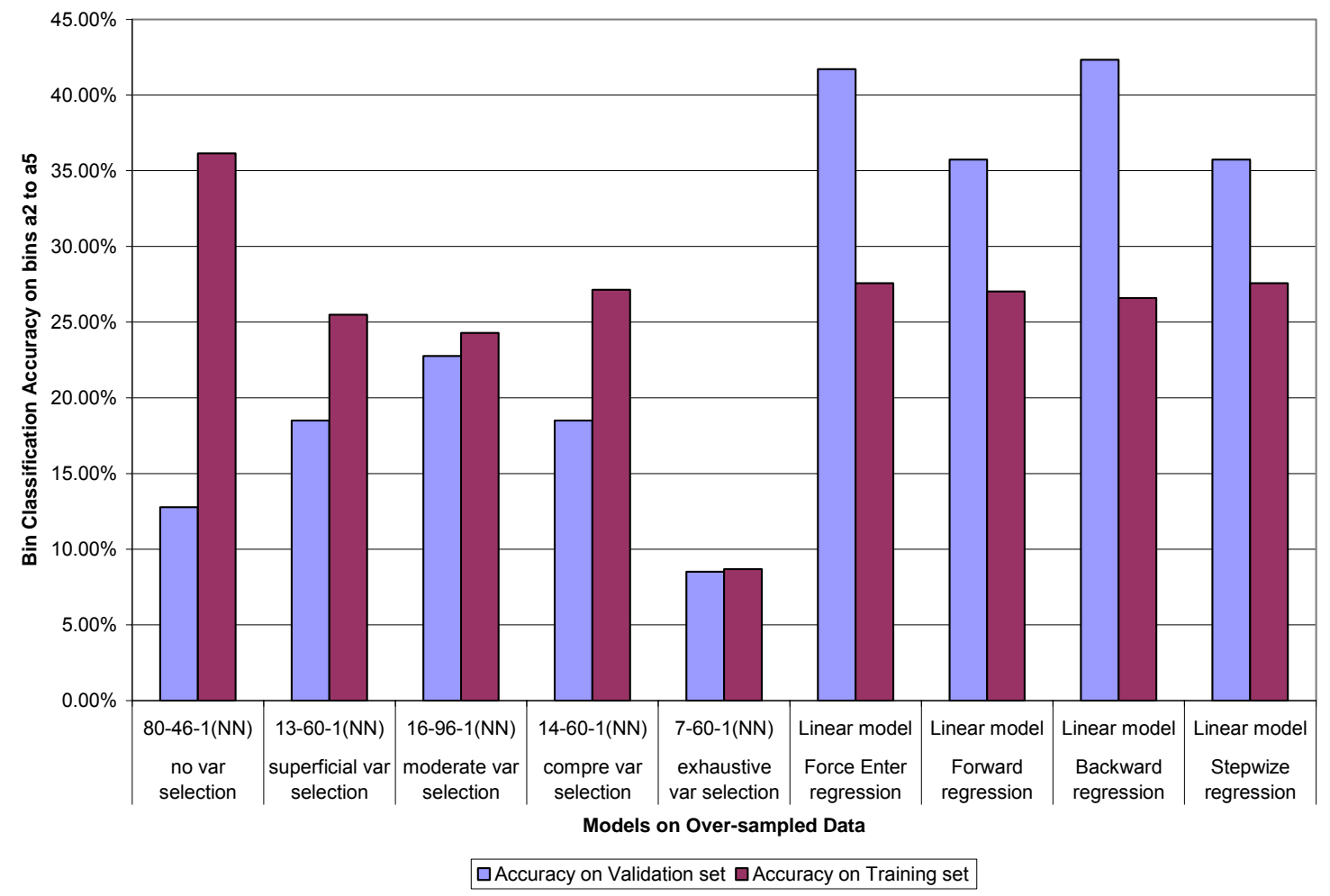

Figure 30: Bin Classification Accuracy on Moderate Range of change in VTR (a2 to a5) for validation \& training set (Different models on Over-sampled data) 


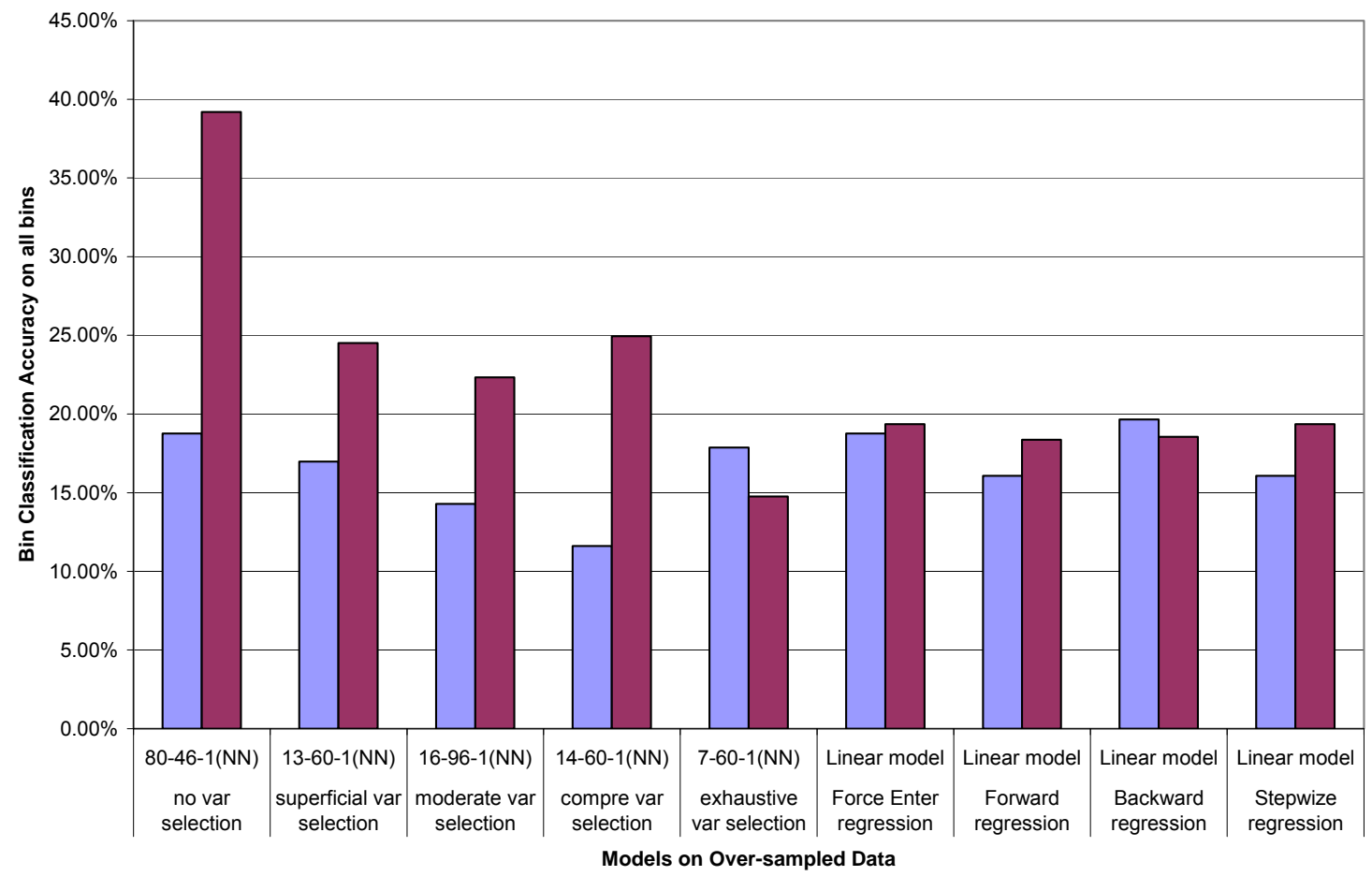

$\square$ Accuracy on Validation Set $\square$ Accuracy on Training Set

Figure 31: Bin Classification Accuracy on Full Range of change in VTR (all bins) for validation \& training set (Different models on Over-sampled data)

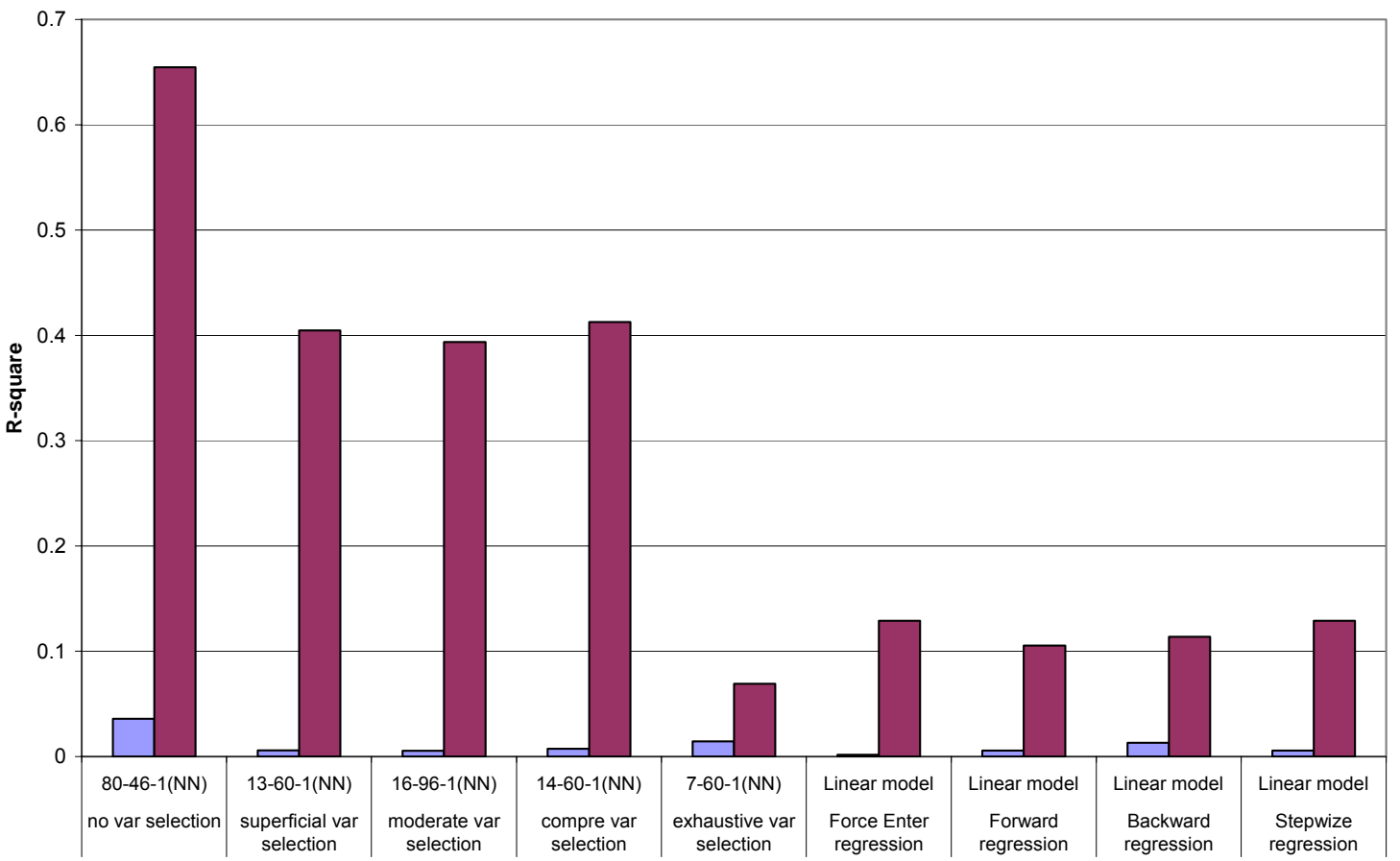

Models on Over-sampled Data

$\square R$-square on Validation Set $\square R$-square on Training Set

Figure 32: R-square for validation \& training set (Different models on Over-sampled data) 
The variables selected from the over-sampled datasets by these models are shown in Table 20

Table 20: Variables selected by different models on Tucson over-sampled data

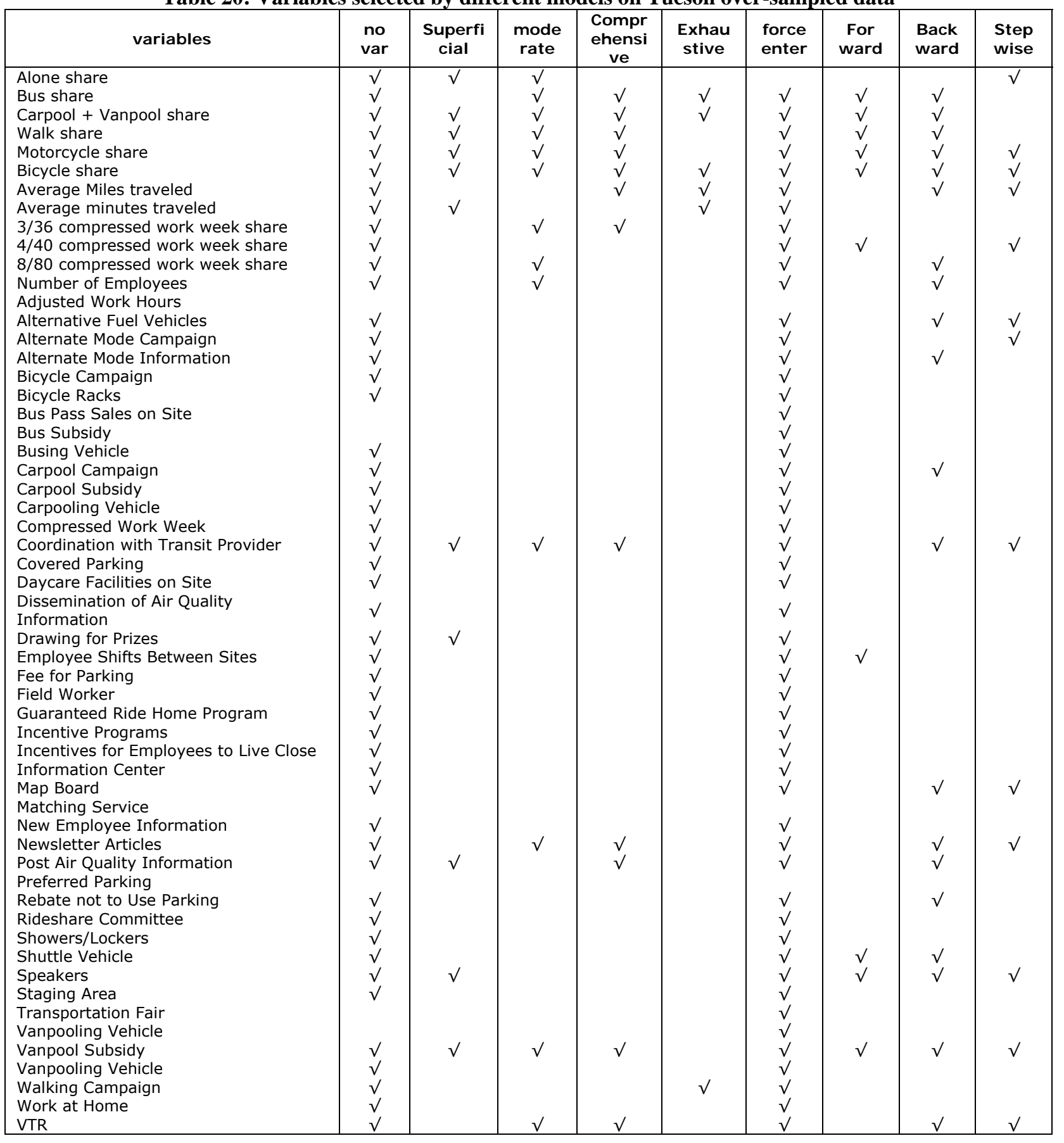


It can be seen from Table 20, that the variables selected by the neural network models with variable selection are not really the key incentives and therefore these models are deemed as unsuitable models. A neural network model built without variable selection (M2) was able to get the best R-square value with second best 'bin classification accuracy on full range of change in VTR'. But, it performed poorly in the required bins a2 to a5.

All regression models were able to get very good 'bin classification accuracy on moderate range of change in VTR' with backward regression model (M1) getting the best 'bin classification accuracy on full range of change in VTR' with second best R-square value. The detailed bins accuracies and scatter plots are shown in Table 21 and Figures 33 and 34.

Table 21: Detailed accuracies on bins: Tucson over-sampled data.

\begin{tabular}{|c|c|c|c|c|c|c|c|c|c|c|c|c|}
\hline & \multirow[b]{2}{*}{ range } & \multirow{2}{*}{$\begin{array}{c}\text { \# of } \\
\text { records }\end{array}$} & \multicolumn{8}{|c|}{ Bin ranges over Change in Vehicle Trip Rate (Delta_VTR) } & \multirow{6}{*}{$\begin{array}{c}\text { Weighted } \\
\text { Avg on } \\
\text { a2 to a5 }\end{array}$} & \multirow{5}{*}{ R-square } \\
\hline & & & $>-7$ & $\begin{array}{c}{[-7 \text { to }-} \\
4.5) \\
\end{array}$ & $\begin{array}{c}{\left[\begin{array}{c}-4.5 \text { to } \\
-3)\end{array}\right.} \\
\end{array}$ & $\begin{array}{c}{[-3 \text { to }-} \\
1.5) \\
\end{array}$ & $\begin{array}{c}{[-1.5 \text { to }} \\
0)\end{array}$ & $\begin{array}{l}\text { [0 to } \\
1.5)\end{array}$ & $\begin{array}{c}\text { [1.5 to } \\
3.5)\end{array}$ & $3.5>=$ & & \\
\hline & Bin Number & & a1 & a2 & a3 & a4 & a5 & a6 & a7 & a8 & & \\
\hline & Validation & 112 & 4 & 6 & 6 & 13 & 19 & 25 & 22 & 17 & & \\
\hline & Training & 1009 & 37 & 52 & 56 & 116 & 171 & 222 & 200 & 155 & & \\
\hline & & & & & & & & & & & & \\
\hline M1 & Exact Validation & $19.64 \%$ & $0.00 \%$ & $25.00 \%$ & $10.00 \%$ & $47.06 \%$ & $58.82 \%$ & $9.52 \%$ & $0.00 \%$ & $0.00 \%$ & $42.34 \%$ & 0.013 \\
\hline & Exact Training & $18.55 \%$ & $0.00 \%$ & $1.81 \%$ & $26.98 \%$ & $46.37 \%$ & $28.76 \%$ & $17.21 \%$ & $7.73 \%$ & $3.66 \%$ & $26.59 \%$ & 0.114 \\
\hline & One-off Validation & $41.07 \%$ & $0.00 \%$ & $25.00 \%$ & $50.00 \%$ & $94.12 \%$ & $82.35 \%$ & $33.33 \%$ & $15.79 \%$ & $0.00 \%$ & $75.53 \%$ & \\
\hline & One-Off Training & $48.82 \%$ & $8.45 \%$ & $25.79 \%$ & $66.05 \%$ & $91.13 \%$ & $73.45 \%$ & $55.81 \%$ & $25.41 \%$ & $10.98 \%$ & & \\
\hline M2 & Exact Validation & $18.75 \%$ & $0.00 \%$ & $0.00 \%$ & $0.00 \%$ & $17.65 \%$ & $17.65 \%$ & $19.05 \%$ & $21.05 \%$ & $35.00 \%$ & $12.77 \%$ & 0.036 \\
\hline & Exact Training & $39.21 \%$ & $67.61 \%$ & $47.06 \%$ & $34.88 \%$ & $33.47 \%$ & $29.65 \%$ & $30.23 \%$ & $33.70 \%$ & $49.39 \%$ & $36.15 \%$ & 0.655 \\
\hline & One-off Validation & $50.00 \%$ & $20.00 \%$ & $0.00 \%$ & $50.00 \%$ & $29.41 \%$ & $64.71 \%$ & $66.67 \%$ & $52.63 \%$ & $50.00 \%$ & $43.62 \%$ & \\
\hline & One-Off Training & $78.35 \%$ & $88.73 \%$ & $87.33 \%$ & $77.67 \%$ & $79.03 \%$ & $75.22 \%$ & $73.95 \%$ & $71.82 \%$ & $74.39 \%$ & & \\
\hline
\end{tabular}


Actual Delta VTR vs. Predicted Delta VTR

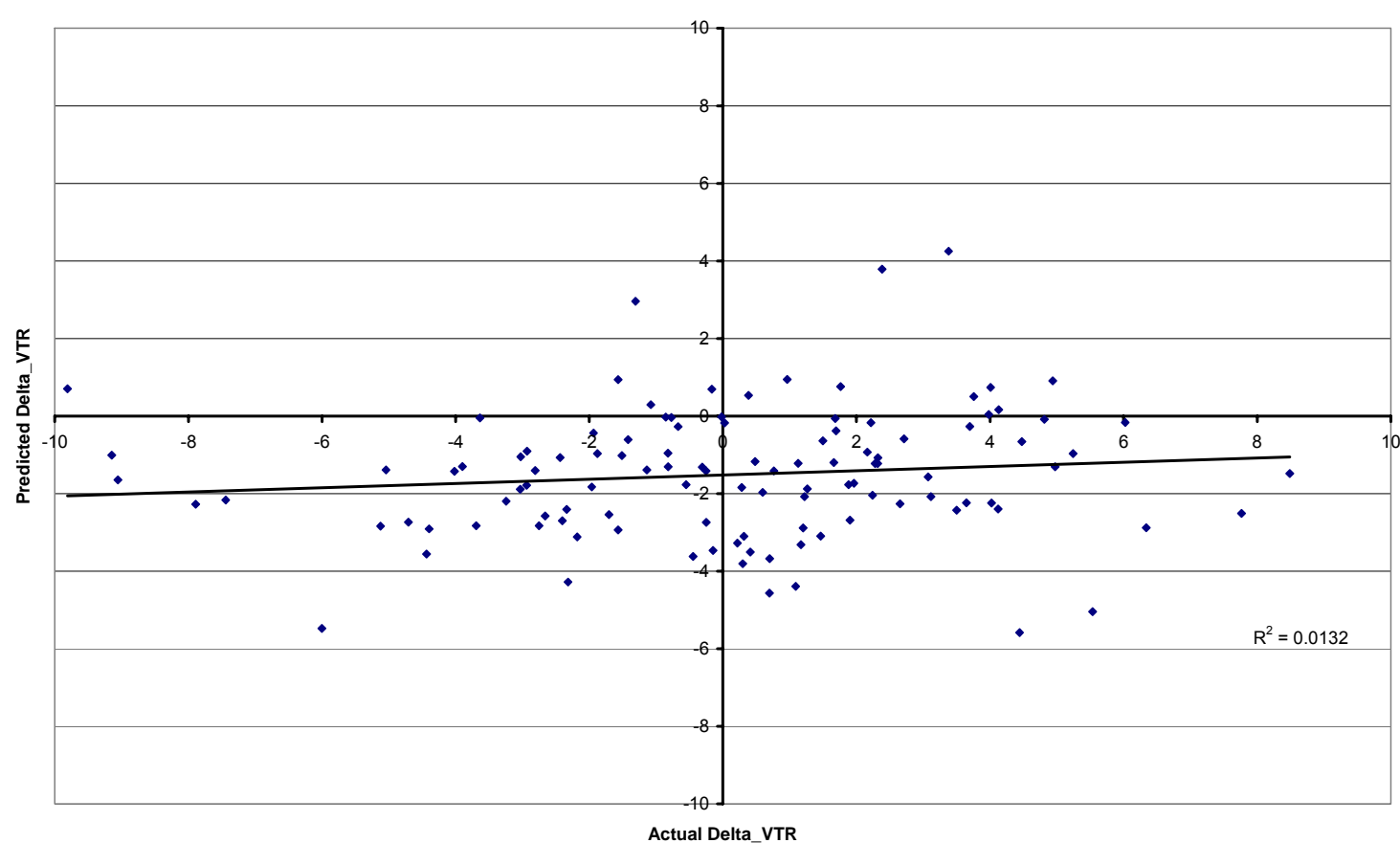

Figure 33: Scatter plot for linear backward regression model

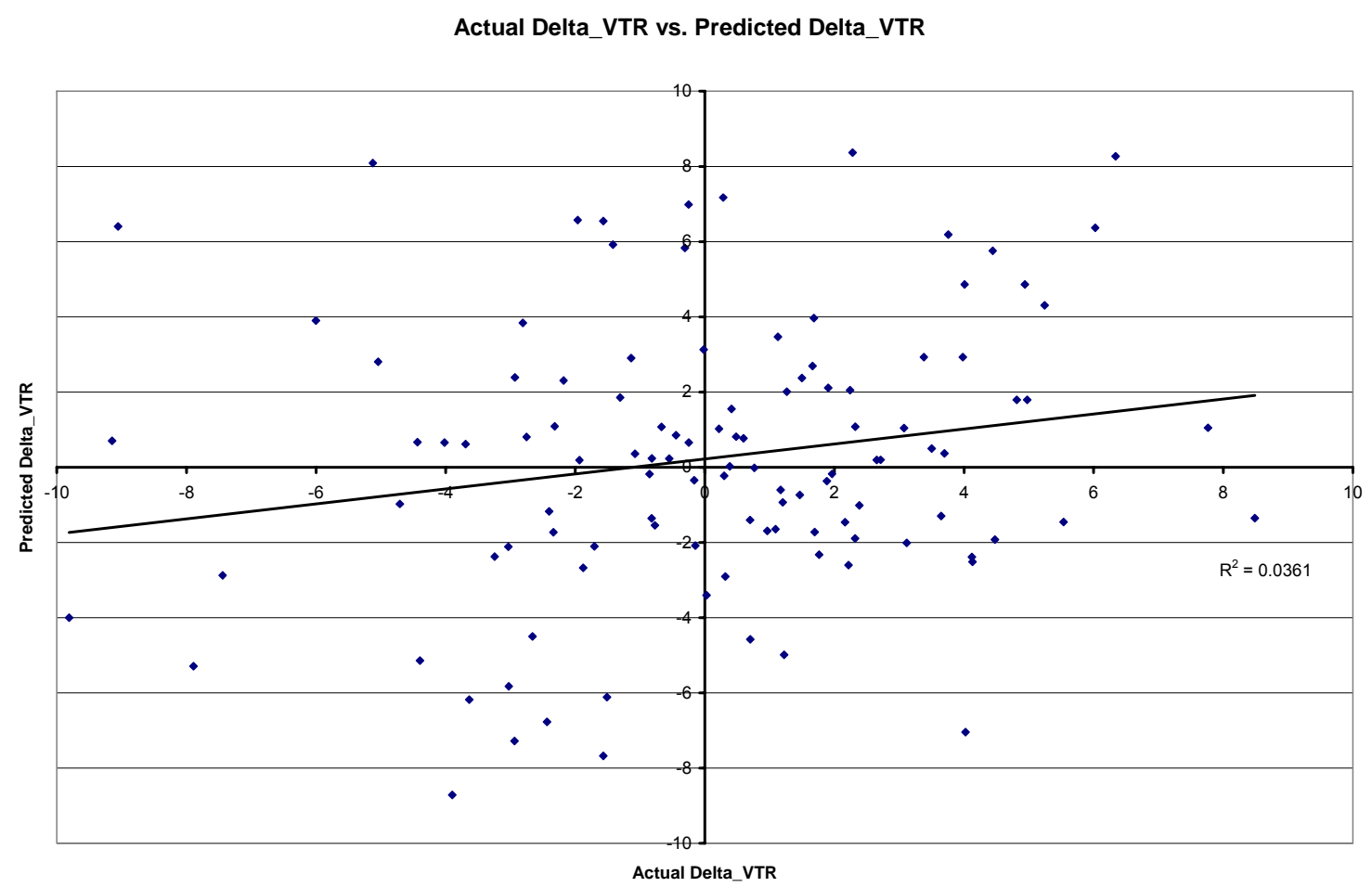

Figure 34: Scatter plot for neural network model built with no variable selection 
It is apparent from the two scatter plots that the backward regression model has a very high tendency to predict negative changes in VTR, whereas the neural network model seems much more balanced, but still getting some predictions way off from the actual change in VTR. Also the neural network model gets better accuracy on the training set than the linear models and so in considered better than the linear model.

Table 22: Variable and backward regression coefficients

\begin{tabular}{|l|r|r|}
\hline \multicolumn{1}{|c|}{ Variable } & coefficients & t - value \\
\hline (Constant) & -35.893 & -2.923 \\
Bus share & 30.707 & 2.315 \\
Carpool + Vanpool share & 29.177 & 4.162 \\
walk share & 38.802 & 2.834 \\
Motorcycle share & 73.305 & 4.810 \\
Bicycle share & 97.354 & 5.230 \\
Average Miles traveled & 0.008 & 1.897 \\
8/80 compressed work week share & 0.007 & 1.790 \\
No. of employees & 0.000 & 1.913 \\
Post Air Quality Information & 0.711 & 2.085 \\
Bus Pass Sales on Site & -0.476 & -1.694 \\
Alternative Fuel Vehicles & -1.099 & -1.687 \\
Compressed Work Week & 0.797 & 2.977 \\
Map Board & -0.696 & -2.349 \\
Preferred Parking & 1.075 & 3.807 \\
Rideshare Committee & 0.964 & 3.424 \\
Staging Area & -1.552 & -1.936 \\
Shuttle Vehicle & -1.494 & -2.241 \\
Showers/Lockers & 0.763 & 2.851 \\
Vanpooling Vehicle & -3.578 & -2.983 \\
Vehicle trip rate & 0.292 & 2.352 \\
\hline
\end{tabular}


Equivalent grouped incentives models were also built on this over-sampled data. Table 23 and Figures 35, 36, and 37 shows the accuracies and the $\mathrm{R}$-square values of the neural network and the regression models built using no variable selection.

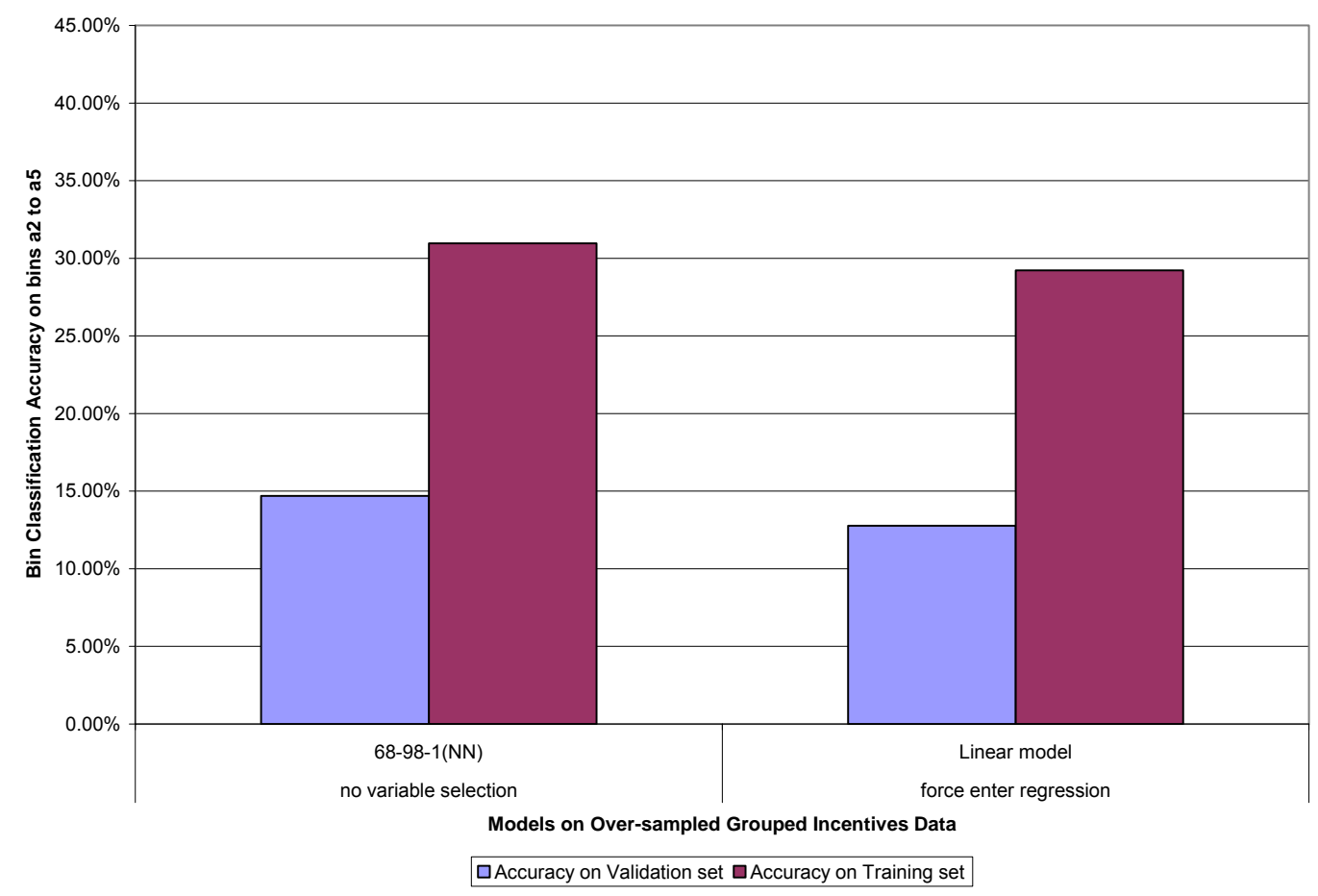

Figure 35: Bin Classification Accuracy on Moderate Range of change in VTR (a2 to a5) for validation \& training set (Different models on Over-sampled Grouped Incentive data)

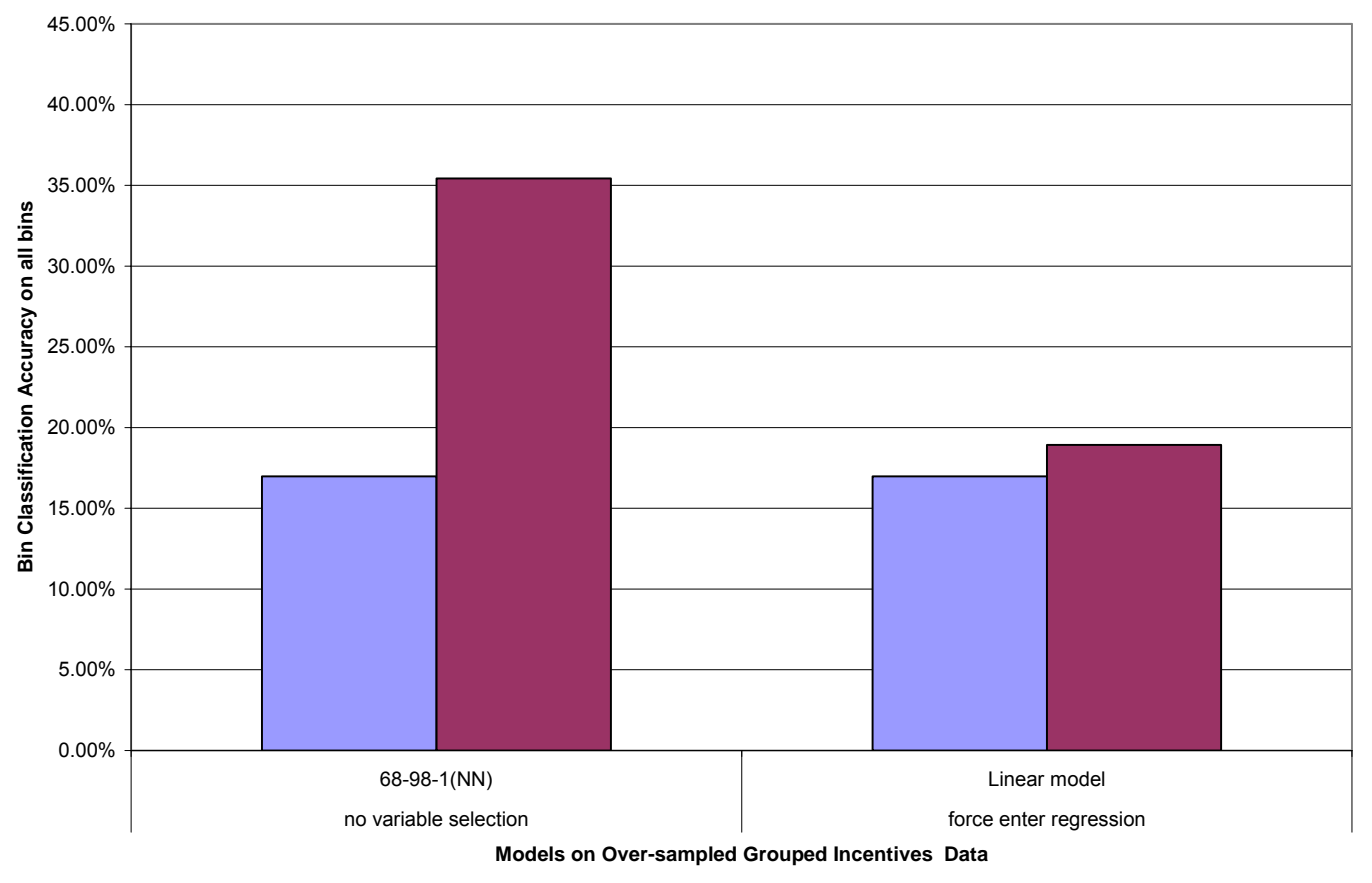

$\square$ Accuracy on Validation Set $\square$ Accuracy on Training Set

Figure 36: Bin Classification Accuracy on Full Range of change in VTR (all bins) for validation \& training set (Different models on Over-sampled Grouped Incentive data) 


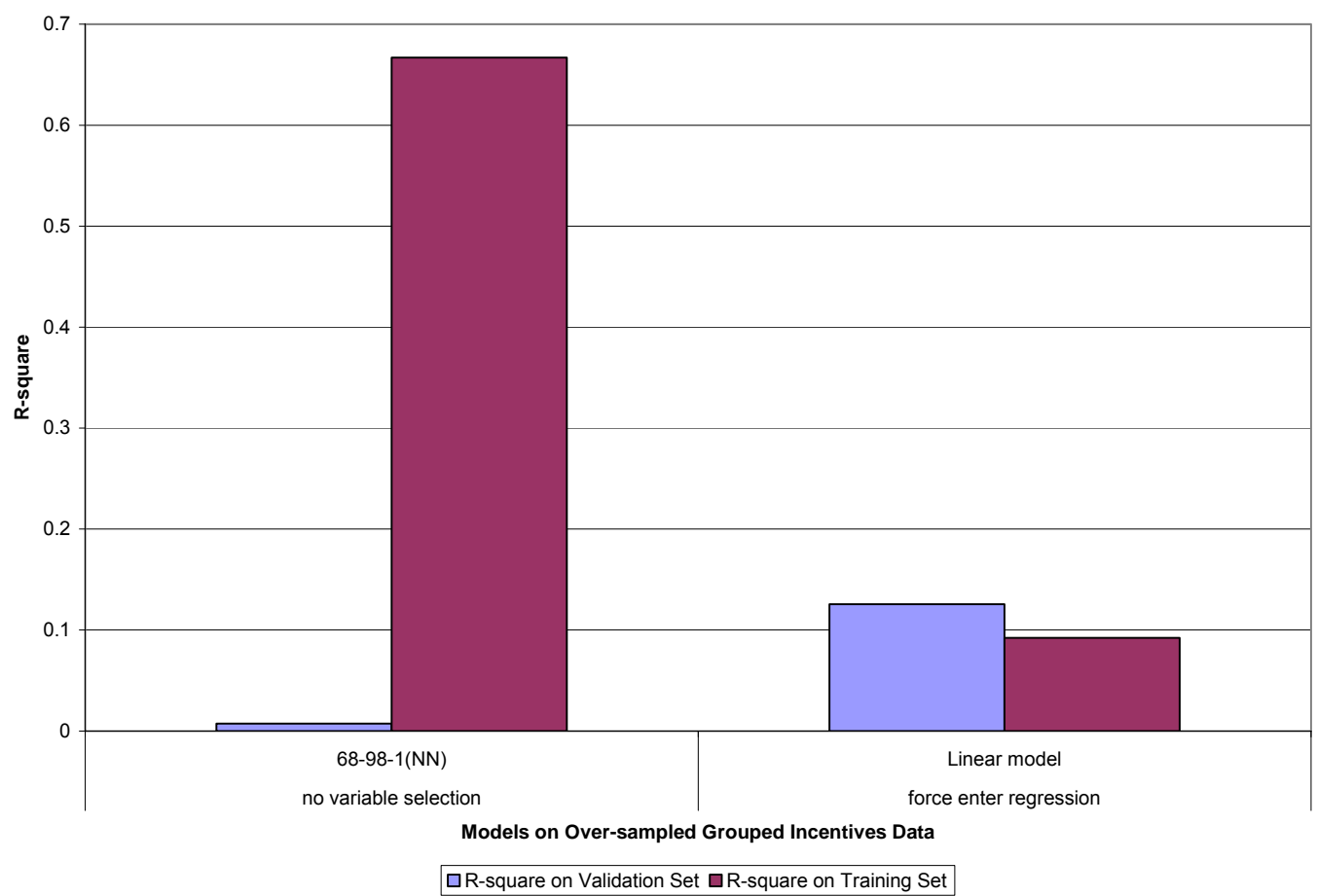

Figure 37: R-square for validation \& training set (Different models on Over-sampled Grouped Incentive data)

For the validation set, it can be seen that both models have equivalent 'bin classification accuracy on full range of change in VTR' (16.96 percent), with the neural network model (M1) obtaining better accuracy in 'bin classification on moderate range of change in VTR' (14.68 percent) and the forced enter regression model (M2) having better R-square value $(0.126)$. The detailed bin accuracies are shown in Table 23 and the scatter plots are shown in Figures 38 and 39.

Table 23: Detailed accuracies on bins

\begin{tabular}{|c|c|c|c|c|c|c|c|c|c|c|c|c|}
\hline & \multirow[b]{2}{*}{ range } & \multirow{2}{*}{$\begin{array}{c}\text { \# of } \\
\text { records }\end{array}$} & \multicolumn{8}{|c|}{ Bin ranges over Change in Vehicle Trip Rate (Delta_VTR) } & \multirow{6}{*}{$\begin{array}{c}\text { Weighted } \\
\text { Avg on } \\
\text { a2 to a5 }\end{array}$} & \multirow{5}{*}{ R-square } \\
\hline & & & $>-7$ & $\begin{array}{c}{[-7 \text { to }-} \\
4.5)\end{array}$ & $\begin{array}{c}{\left[\begin{array}{c}-4.5 \\
-3)\end{array}\right.} \\
\text { to }\end{array}$ & $\begin{array}{c}{[-3 \text { to }-} \\
1.5)\end{array}$ & $\begin{array}{c}{[-1.5 \text { to }} \\
0)\end{array}$ & $\begin{array}{l}\text { [0 to } \\
1.5)\end{array}$ & {$\left[\begin{array}{c}1.5 \text { to } \\
3.5)\end{array}\right.$} & $3.5>=$ & & \\
\hline & Bin Number & & a1 & a2 & a3 & a4 & a5 & A6 & a7 & a8 & & \\
\hline & Validation & 112 & 4 & 6 & 6 & 13 & 19 & 25 & 22 & 17 & & \\
\hline & Training & 1009 & 37 & 52 & 56 & 116 & 171 & 222 & 200 & 155 & & \\
\hline & & & & & & & & & & & & \\
\hline M1 & Exact Validation & $16.96 \%$ & $40.00 \%$ & $0.00 \%$ & $10.00 \%$ & $17.65 \%$ & $17.65 \%$ & $19.05 \%$ & $5.26 \%$ & $25.00 \%$ & $14.68 \%$ & 0.007 \\
\hline & Exact Training & $35.42 \%$ & $67.61 \%$ & $41.18 \%$ & $29.30 \%$ & $27.20 \%$ & $26.67 \%$ & $25.82 \%$ & $30.22 \%$ & $50.61 \%$ & $30.96 \%$ & 0.667 \\
\hline & One-off Validation & $36.61 \%$ & $40.00 \%$ & $0.00 \%$ & $30.00 \%$ & $41.18 \%$ & $41.18 \%$ & $47.62 \%$ & $31.58 \%$ & $30.00 \%$ & $35.53 \%$ & \\
\hline & One-Off Training & $76.24 \%$ & $97.18 \%$ & $71.04 \%$ & $84.19 \%$ & $73.60 \%$ & $74.22 \%$ & $72.77 \%$ & $69.23 \%$ & $73.78 \%$ & & \\
\hline M2 & Exact Validation & $16.96 \%$ & $0.00 \%$ & $0.00 \%$ & $0.00 \%$ & $5.88 \%$ & $29.41 \%$ & $42.86 \%$ & $10.53 \%$ & $10.00 \%$ & $12.77 \%$ & 0.126 \\
\hline & Exact Training & $18.92 \%$ & $0.00 \%$ & $1.81 \%$ & $26.98 \%$ & $50.40 \%$ & $34.96 \%$ & $13.02 \%$ & $3.31 \%$ & $3.05 \%$ & $29.23 \%$ & 0.092 \\
\hline & One-off Validation & $50.89 \%$ & $0.00 \%$ & $0.00 \%$ & $0.00 \%$ & $35.29 \%$ & $100.00 \%$ & $95.24 \%$ & $52.63 \%$ & $20.00 \%$ & $48.94 \%$ & \\
\hline & One-Off Training & $47.77 \%$ & $2.82 \%$ & $12.67 \%$ & $68.84 \%$ & $95.16 \%$ & $80.97 \%$ & $54.88 \%$ & $20.99 \%$ & $9.15 \%$ & & \\
\hline
\end{tabular}




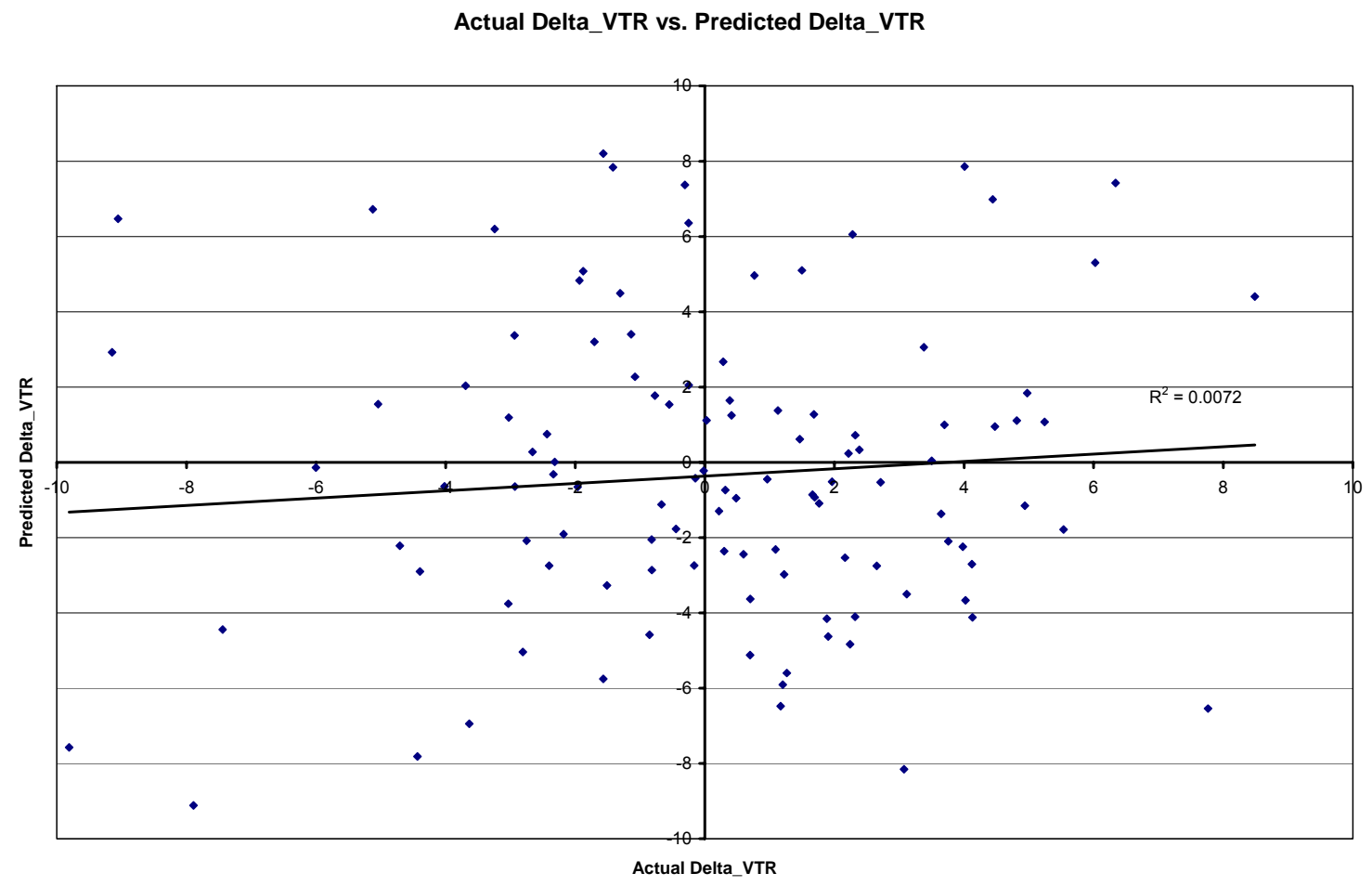

Figure 38: Scatter plot for neural network model

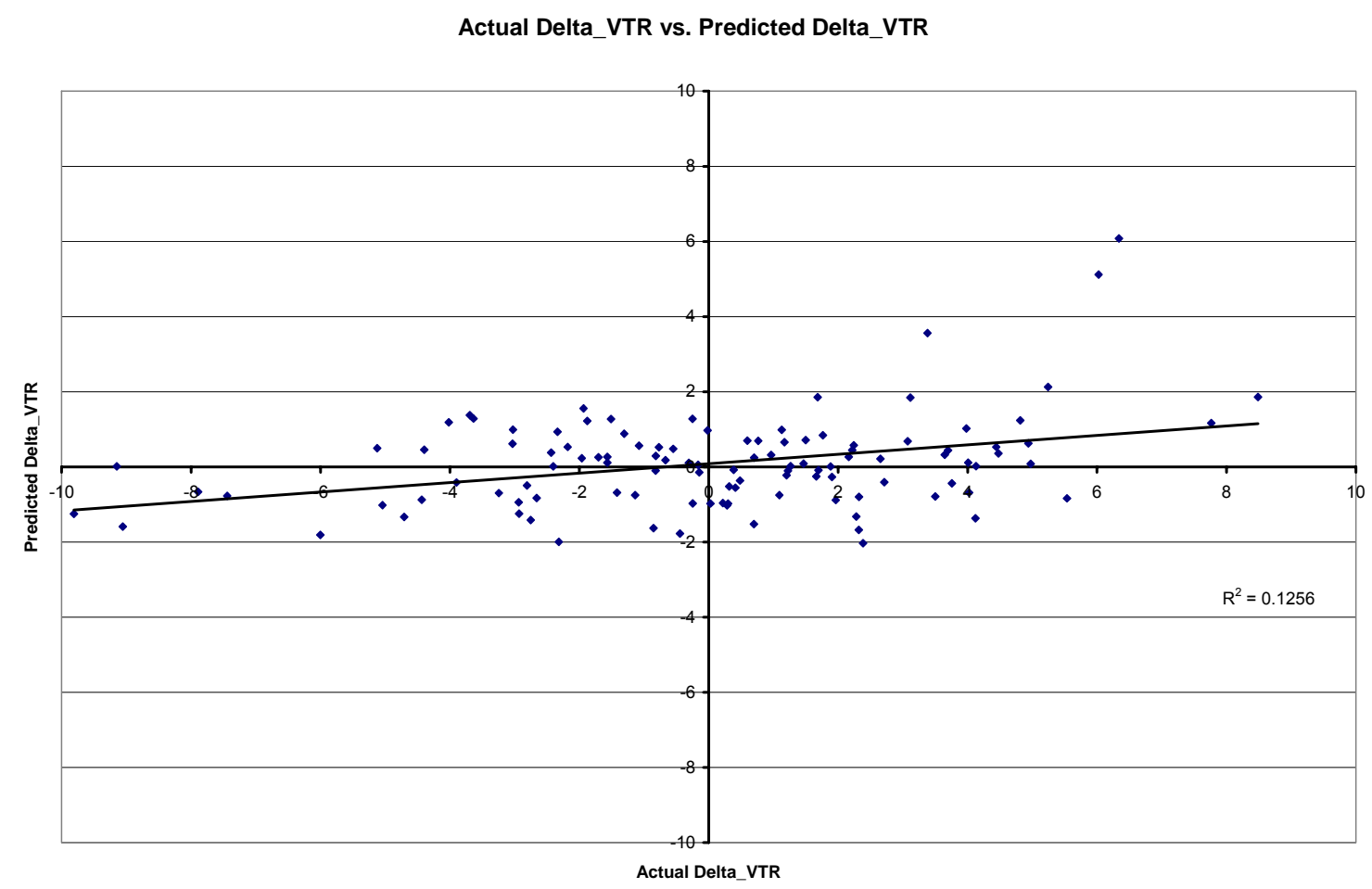

Figure 39: Scatter plot for linear forced enter regression model 
The scatter plots in Figures 38 and 39 show that both the forced enter regression model and the neural net model with no variable selection are balanced in predicting the changes in VTR with regression model predicting smaller changes in VTR as opposed to neural network predicting large changes in VTR close to actual changes in VTR. The neural network model is able to get much better performance than linear regression model on training set and so can be considered better than the regression model.

The regression coefficients for the model are shown in Table 24.

Table 24: Variable and forced enter regression coefficients

\begin{tabular}{|l|r|r|}
\hline \multicolumn{1}{|c|}{ Variables } & coefficients & t - value \\
\hline (Constant) & 22.498 & 0.462 \\
Bus share & -29.515 & -0.577 \\
Carpool + Vanpool share & 3.275 & 0.121 \\
walk share & -5.690 & -0.105 \\
Motorcycle share & -19.791 & -0.352 \\
Bicycle share & -52.474 & -0.729 \\
Average Miles traveled & -0.041 & -1.622 \\
Average minutes traveled & 0.252 & 1.045 \\
3/36 compressed work week share & -0.014 & -0.067 \\
4/40 compressed work week share & -2.557 & -2.739 \\
8/80 compressed work week share & 0.007 & 1.213 \\
No. of employees & 0.002 & 2.614 \\
facilities \& amenities(grouped incentives) & -1.561 & -0.910 \\
Guaranteed ride home programs(grouped incentives) & -0.326 & -0.407 \\
flexible timing (grouped incentives) & -0.900 & -1.232 \\
financial incentives(grouped incentives) & -0.591 & -0.343 \\
Parking management (grouped incentives) & -3.646 & -1.627 \\
Telecommuting (grouped incentives) & -1.259 & -1.550 \\
Compressed work week(grouped incentives) & 0.791 & 0.936 \\
Vanpool vehicles(grouped incentives) & 1.097 & 0.778 \\
onsite incentives(grouped incentives) & 1.425 & 1.630 \\
Non financial (grouped incentives) & 0.199 & 0.161 \\
commuter tax benefit incentives(grouped incentives) & -1.735 & -1.641 \\
Vehicle trip rate & -0.235 & -0.480 \\
\hline
\end{tabular}

When neural network model built on grouped incentives with over-sampled data is compared with the neural network model built on ungrouped individual incentives with over-sampled data (Table 21 and 23), it can be seen that the model on grouped incentives has got accuracy distributed over all bins as against the other model for which the accuracy on bins a1, a2 and a3 is zero. Looking at other performance measures which are not much significantly different, it can be said that that grouped incentive model will be more preferred due to its simplicity 


\section{Recommended Model}

None of the models were exceptionally better than the other models. All regression models were able to predict small changes in VTR as compared to the actual change in VTR. This shortcoming was not desirable. The two best candidate models were the neural network models without variable selection built, one with the ungrouped incentive variables on the full sample data and the other with the grouped incentives on the oversampled data.

The reasons the neural network model built without variable selection on the full sample ungrouped incentive data was considered as candidate was (Table 21-M2)

1. It has the best 'bin classification accuracy on full range of change in VTR' of $20.54 \%$ which is much better than the random choice accuracy of $12.5 \%$

2. It has 'bin classification accuracy on moderate range of change in VTR' of $16.6 \%$

3. It has the 'R-square' value of 0.022

4. It includes all the variables in predicting change in VTR

The reasons the neural network model built without variable selection on the over-sampled grouped incentive data was considered as candidate was (Table 23-M1)

1. It is built on a simple grouped incentives variable set

2. It has 'bin classification accuracy on full range of change in VTR' of $16.96 \%$ which is better than the random choice accuracy of $12.5 \%$

3. It has 'bin classification accuracy on moderate range of change in VTR' of $14.68 \%$

4. It has a 'R-square' value of 0.007

Looking at the performance measures of both models, the neural network model built without variable selection on the full sample ungrouped incentive data can be said to be the recommended model. Also though is the model is not built on simple grouped incentives, it at least include all the incentives in predicting the changes in VTR. 


\section{WASHI NGTON MODEL}

\section{Phase I : Washington Full Sample Data}

The Washington dataset consisted of 1,414 total examples. Approximately ten percent of the dataset was left aside as validation set while the rest became training/testing set. For all of the Washington models the testing set was ten percent of the random data of the training/testing set. The eight bin ranges and the number of examples in each bin for validation and training/testing set are shown in Table 27.

Table 25: Washington Full Sample Data - No. of Records in bins

\begin{tabular}{|l|c|c|c|c|c|c|c|c|c|}
\hline & & \multicolumn{7}{|c|}{ Bin ranges over Change in Vehicle Trip Rate (Delta_VTR) } \\
\cline { 3 - 10 } Range & \# of records & $\mathbf{> - 7}$ & [- 7 to - 4.5) & [- - 4.5 to - 3) & [- - to -1.5) & [-1.5 to 0) & [0 to 1.5) & [1.5 to 3.5) & 3.5 >= \\
\hline Bin Number & & $\mathbf{a 1}$ & $\mathbf{a 2}$ & $\mathbf{a 3}$ & $\mathbf{a 4}$ & $\mathbf{a 5}$ & $\mathbf{a 6}$ & $\mathbf{a 7}$ & $\mathbf{a 8}$ \\
Validation & $\mathbf{1 4 4}$ & 15 & 15 & 15 & 17 & 20 & 20 & 21 & 21 \\
Training & $\mathbf{1 3 0 0}$ & 136 & 132 & 132 & 152 & 182 & 184 & 190 & 192 \\
\hline
\end{tabular}

Neural network models were built of data containing the entire variable set (105 variables) with different variable selections. This dataset contained variables such as shares of employee's incentive preference and type of work they performed. The employers might not be able to provide this information about their employees. So these variables with a few other insignificant variables were removed from the data. The models on the data containing these variables were built to study the contribution of these employee preference variables in predicting the change in VTR.

A neural network model with no variable selection and all different types of regression models were built on this condensed data. Figures 40,41, and 42 show the different performance measures for all of these models. 


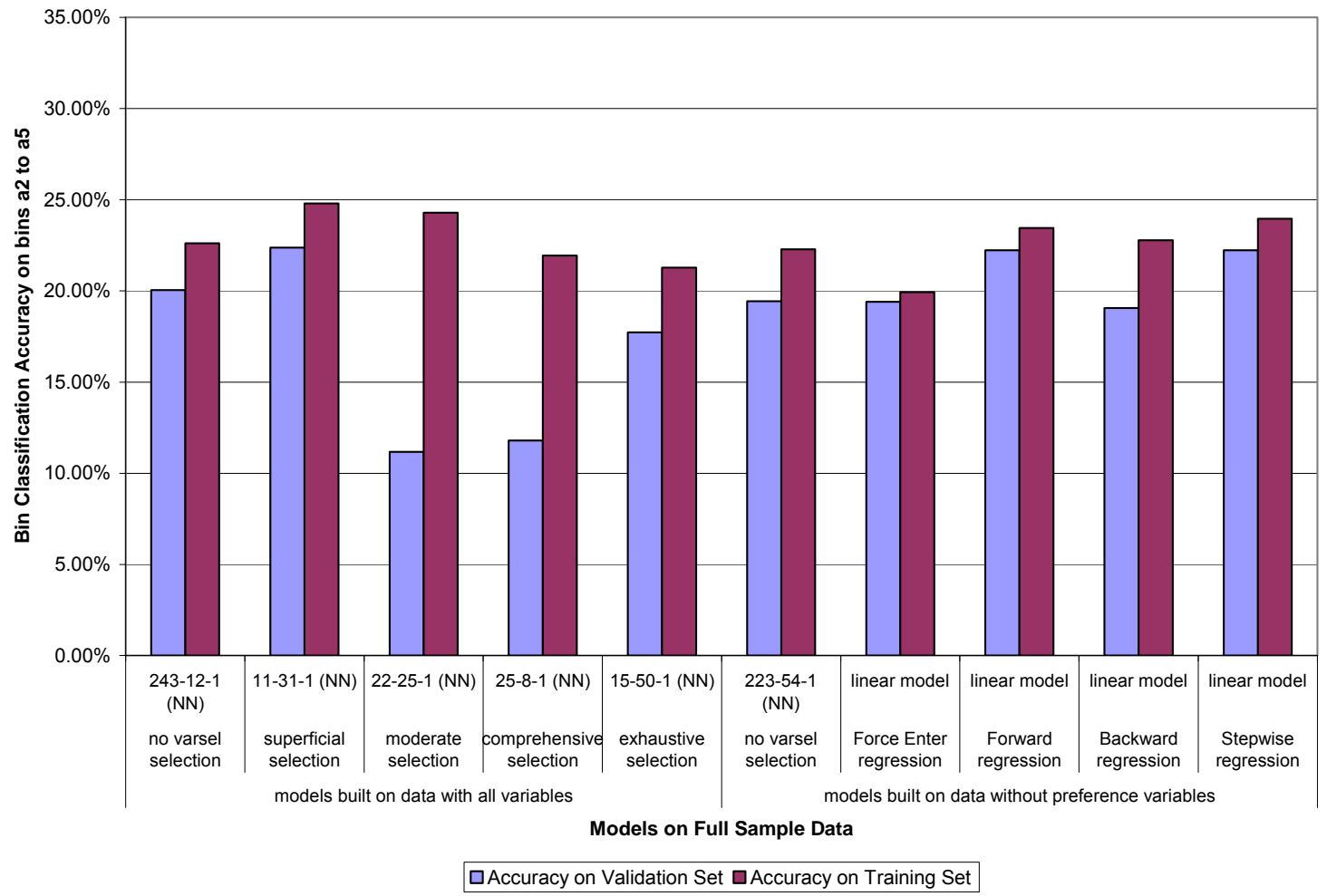

Figure 40: Bin Classification Accuracy on Moderate Range of change in VTR (a2 to a5) for validation \& training set (Different models on Full sample data)

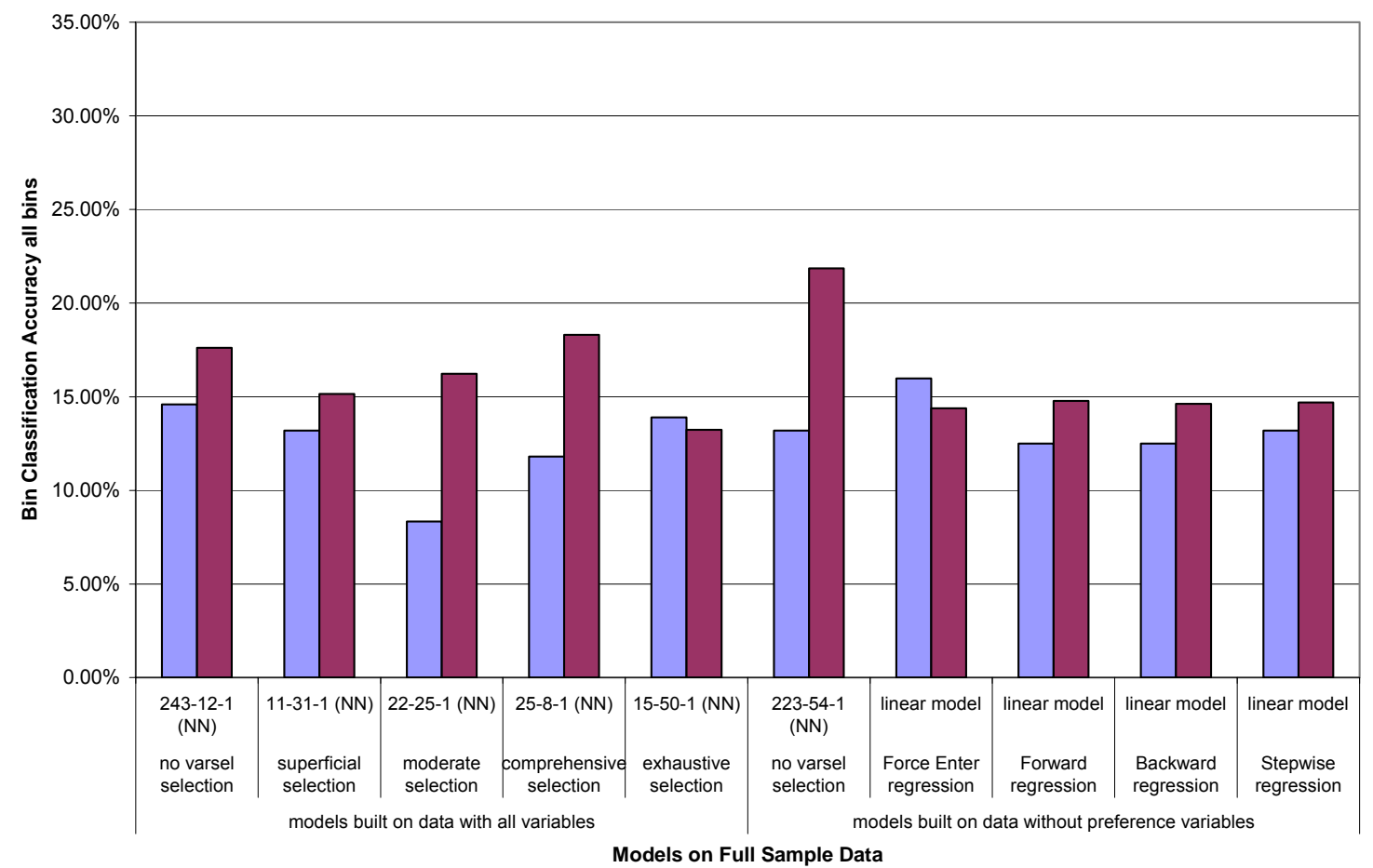

口Accuracy on Validation Set $\square$ Accuracy on Training Set

Figure 41: Bin Classification Accuracy on Full Range of change in VTR (all bins) for validation \& training set (Different models on Full sample data) 


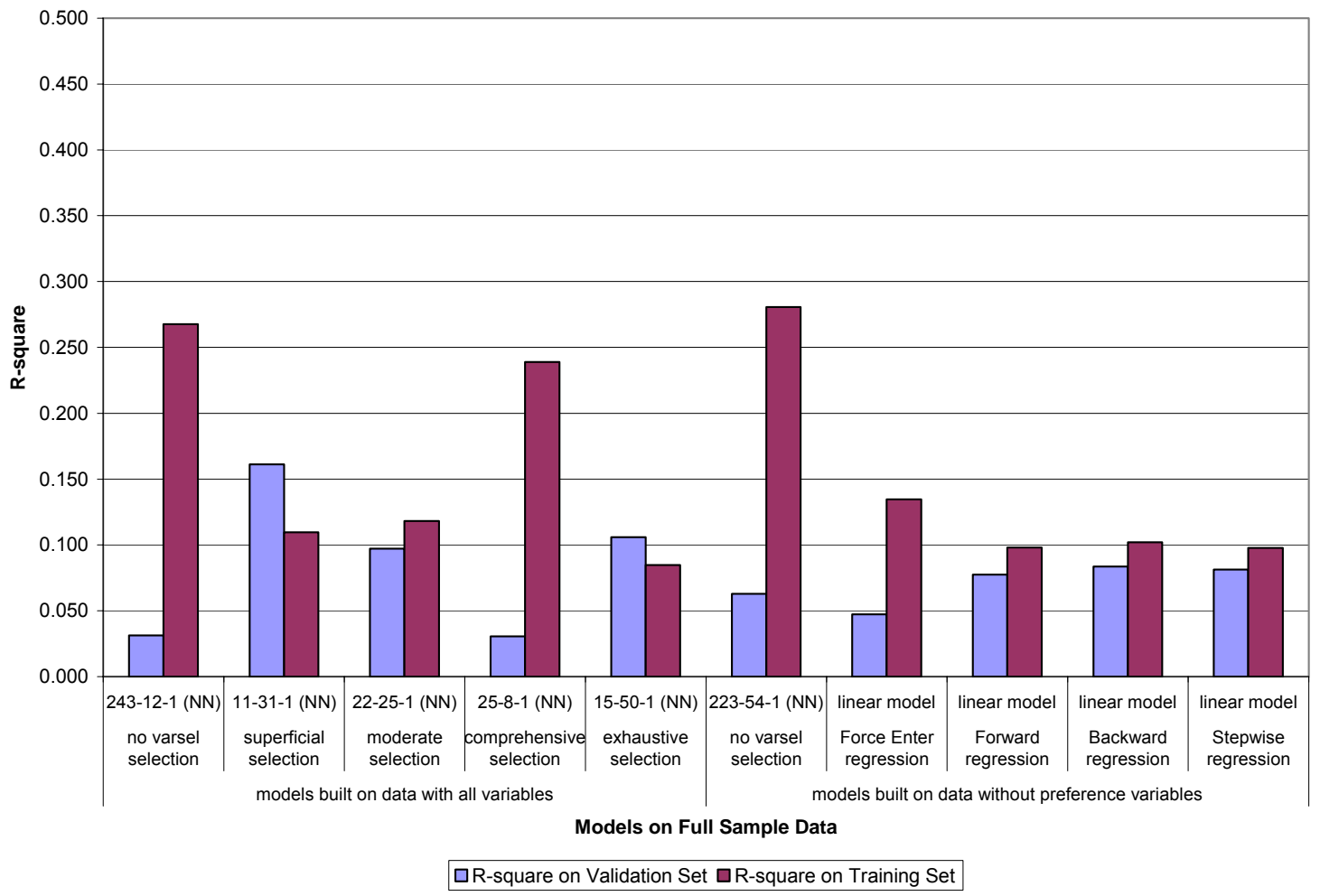

Figure 42: R-square for validation \& training set (Different models on Full sample data)

\section{Table 28 shows the variables selected by the different models.}

Column name abbreviations,

$\mathrm{N}$ - Neural network model without variable selection

$S$ - Neural network model with superficial variable selection

M - Neural network model with moderate variable selection

C - Neural network model with comprehensive variable selection

$\mathrm{E}$ - Neural network model with exhaustive variable selection

FE - Linear Forced Enter regression model

FR - Linear Forward regression model

BK - Linear Backward regression model

SP - Linear Stepwise regression model

$\sqrt{ }$ - indicates the selection of the variable

'-' - indicates the variable was not present in the data 
Table 26: Variables selected by different models on Full Sample data

\begin{tabular}{|c|c|c|c|c|c|c|c|c|c|c|}
\hline \multirow[t]{2}{*}{ Variables } & \multicolumn{5}{|c|}{ NN models on data with all variables } & \multicolumn{5}{|c|}{$\begin{array}{l}\text { Models on data without preference } \\
\text { and other insignificant variables }\end{array}$} \\
\hline & $\mathbf{N}$ & $\mathbf{S}$ & $\mathbf{M}$ & C & $\mathbf{E}$ & $\mathbf{N}$ & FE & FR & BK & SP \\
\hline Non-profit organization & $\sqrt{ }$ & & & & & - & - & - & - & - \\
\hline Agriculture organization & & & & & & - & - & - & - & - \\
\hline Finance organization & $\sqrt{ }$ & & & & & - & - & - & - & - \\
\hline Info services organization & $\sqrt{ }$ & & & & & - & - & - & - & - \\
\hline Health organization & $\sqrt{ }$ & & & & & $\sqrt{ }$ & $\sqrt{ }$ & & & \\
\hline Retail organization & $\sqrt{ }$ & & & & & $\sqrt{ }$ & $\sqrt{ }$ & & & \\
\hline Manufacturing organization & $\sqrt{ }$ & & & & & $\sqrt{ }$ & $\sqrt{ }$ & & $\sqrt{ }$ & \\
\hline Services organization & $\sqrt{ }$ & & & & & - & - & - & - & - \\
\hline Public utilities organization & $\sqrt{ }$ & & $\sqrt{ }$ & & & - & - & - & - & - \\
\hline Construction organization & & & & & & - & - & - & - & - \\
\hline Transportation organization & $\sqrt{ }$ & & & & & - & - & - & - & - \\
\hline Government organization & $\sqrt{ }$ & & $\sqrt{ }$ & & & - & - & - & - & - \\
\hline Other organization & $\sqrt{ }$ & & & & & $\sqrt{ }$ & $\sqrt{ }$ & & $\sqrt{ }$ & \\
\hline Offered to All & $\sqrt{ }$ & & & & & - & - & - & - & - \\
\hline Union & $\sqrt{ }$ & & & & & $\sqrt{ }$ & $\sqrt{ }$ & & & \\
\hline Shifts & $\sqrt{ }$ & & & & & $\sqrt{ }$ & $\sqrt{ }$ & & & \\
\hline Onsite Parking Spaces & $\sqrt{ }$ & & & & & $\sqrt{ }$ & $\sqrt{ }$ & & & \\
\hline Offsite Parking Spaces & $\sqrt{ }$ & $\sqrt{ }$ & & $\sqrt{ }$ & & $\sqrt{ }$ & $\sqrt{ }$ & & & \\
\hline Leased Onsite Parking Price & $\sqrt{ }$ & & & & $\sqrt{ }$ & $\sqrt{ }$ & $\sqrt{ }$ & & & \\
\hline Leased Offsite Parking Price & $\sqrt{ }$ & & $\sqrt{ }$ & & & - & - & - & - & - \\
\hline Own Onsite Parking & $\sqrt{ }$ & & $\sqrt{ }$ & $\sqrt{ }$ & & $\sqrt{ }$ & $\sqrt{ }$ & $\sqrt{ }$ & $\sqrt{ }$ & $\sqrt{ }$ \\
\hline Onsite Parking Charge & $\sqrt{ }$ & & & & & $\sqrt{ }$ & $\sqrt{ }$ & & & \\
\hline Own Offsite Parking & $\sqrt{ }$ & & & & & $\sqrt{ }$ & $\sqrt{ }$ & & & \\
\hline Offsite Parking Charge & $\sqrt{ }$ & & & $\sqrt{ }$ & & $\sqrt{ }$ & $\sqrt{ }$ & & & \\
\hline Pay Parking Charge & $\sqrt{ }$ & & & & & $\sqrt{ }$ & $\sqrt{ }$ & & & \\
\hline On off parking sub & $\sqrt{ }$ & & & $\sqrt{ }$ & & $\sqrt{ }$ & $\sqrt{ }$ & $\sqrt{ }$ & $\sqrt{ }$ & $\sqrt{ }$ \\
\hline Free Parking $1 / 4$ mile & $\sqrt{ }$ & & & & & $\sqrt{ }$ & $\sqrt{ }$ & & & \\
\hline ETC Additional Training & $\sqrt{ }$ & & & & & $\sqrt{ }$ & $\sqrt{ }$ & & & \\
\hline ETC Onsite & $\sqrt{ }$ & & & & & $\sqrt{ }$ & $\sqrt{ }$ & & & \\
\hline Distribute Info & $\sqrt{ }$ & & & & & $\sqrt{ }$ & $\sqrt{ }$ & & & \\
\hline Post Materials & $\sqrt{ }$ & & & & $\sqrt{ }$ & $\sqrt{ }$ & $\sqrt{ }$ & & & \\
\hline CTR Orientation & $\sqrt{ }$ & & & & & $\sqrt{ }$ & $\sqrt{ }$ & & & \\
\hline CTR Events & $\sqrt{ }$ & & & $\sqrt{ }$ & $\sqrt{ }$ & $\sqrt{ }$ & $\sqrt{ }$ & $\sqrt{ }$ & $\sqrt{ }$ & $\sqrt{ }$ \\
\hline CTR E-mail & $\sqrt{ }$ & & & $\sqrt{ }$ & & $\sqrt{ }$ & $\sqrt{ }$ & & & \\
\hline Articles & $\sqrt{ }$ & & & & & $\sqrt{ }$ & $\sqrt{ }$ & & & \\
\hline Ride match Apps & $\sqrt{ }$ & & & $\sqrt{ }$ & & $\sqrt{ }$ & $\sqrt{ }$ & & & \\
\hline With Paychecks & $\sqrt{ }$ & & & & $\sqrt{ }$ & $\sqrt{ }$ & $\sqrt{ }$ & & & \\
\hline Drawings & $\sqrt{ }$ & $\sqrt{ }$ & & $\sqrt{ }$ & & $\sqrt{ }$ & $\sqrt{ }$ & $\sqrt{ }$ & $\sqrt{ }$ & $\sqrt{ }$ \\
\hline Leave & & & & & & - & - & - & - & - \\
\hline Other Promo & $\sqrt{ }$ & & & & & $\sqrt{ }$ & $\checkmark$ & & & \\
\hline Covered Bike Number & $\sqrt{ }$ & & & & & $\sqrt{ }$ & $\sqrt{ }$ & & & \\
\hline Uncovered Bike Number & $\sqrt{ }$ & & & & & $\sqrt{ }$ & $\sqrt{ }$ & & & \\
\hline Lockers Number & $\sqrt{ }$ & & & & & $\sqrt{ }$ & $\mathrm{V}$ & & & \\
\hline Showers Number & $\sqrt{ }$ & & & $\sqrt{ }$ & & $\sqrt{ }$ & $\sqrt{ }$ & & & \\
\hline Shelters Number & $\sqrt{ }$ & & & & & $\sqrt{ }$ & $\sqrt{ }$ & & & \\
\hline Other Amenities 1 Number & $\sqrt{ }$ & & $\sqrt{ }$ & & & $\sqrt{ }$ & $\sqrt{ }$ & $\sqrt{ }$ & $\sqrt{ }$ & $\sqrt{ }$ \\
\hline Carpool Spaces Number & $\sqrt{ }$ & & & $\sqrt{ }$ & & $\sqrt{ }$ & $\sqrt{ }$ & & & \\
\hline Vanpool Spaces Number & $\sqrt{ }$ & & & & & $\sqrt{ }$ & $\sqrt{ }$ & & & \\
\hline SOV Parking Charge & $\sqrt{ }$ & & & & & $\sqrt{ }$ & $\sqrt{ }$ & & $\sqrt{ }$ & \\
\hline SOV Parking Charge Number & $\sqrt{ }$ & & & $\sqrt{ }$ & & $\sqrt{ }$ & $\sqrt{ }$ & & & \\
\hline Reduced SOVP Number & $\sqrt{ }$ & & & & & $\sqrt{ }$ & $\sqrt{ }$ & & & \\
\hline Transit Subsidy & $\sqrt{ }$ & & $\sqrt{ }$ & & $\sqrt{ }$ & $\sqrt{ }$ & $\sqrt{ }$ & & & \\
\hline Ferry Subsidy & $\sqrt{ }$ & & & & $\sqrt{ }$ & $\sqrt{ }$ & $\sqrt{ }$ & & & \\
\hline Vanpool Subsidy & $\sqrt{ }$ & & & & & $\sqrt{ }$ & $\sqrt{ }$ & & & \\
\hline Carpool Subsidy & $\sqrt{ }$ & & & & & $\sqrt{ }$ & $\sqrt{ }$ & & & \\
\hline Walking Subsidy & $\sqrt{ }$ & & & & & $\sqrt{ }$ & $\sqrt{ }$ & & & \\
\hline Bike Subsidy & $\sqrt{ }$ & & & & & $\sqrt{ }$ & $\sqrt{ }$ & & & \\
\hline Employees on flextime & $\sqrt{ }$ & & & & & $\sqrt{ }$ & $\sqrt{ }$ & & & \\
\hline Employees with GRH & $\sqrt{ }$ & & & & & $\sqrt{ }$ & $\sqrt{ }$ & & & \\
\hline Employees in-house match & $\sqrt{ }$ & & & & & $\sqrt{ }$ & $\sqrt{ }$ & & & \\
\hline Employees public match & $\sqrt{ }$ & & & & & $\sqrt{ }$ & $\sqrt{ }$ & & & \\
\hline
\end{tabular}




\begin{tabular}{|c|c|c|c|c|c|c|c|c|c|c|}
\hline \multirow[t]{2}{*}{ Variables } & \multicolumn{5}{|c|}{ NN models on data with all variables } & \multicolumn{5}{|c|}{$\begin{array}{l}\text { Models on data without preference } \\
\text { and other insignificant variables }\end{array}$} \\
\hline & $\mathbf{N}$ & $\mathbf{s}$ & M & c & $\mathbf{E}$ & $\mathbf{N}$ & FE & FR & BK & SP \\
\hline FV work employees & $\sqrt{ }$ & & & & & $\sqrt{ }$ & $\sqrt{ }$ & & & \\
\hline Number of Employees & $\checkmark$ & & & $\sqrt{ }$ & & $\sqrt{ }$ & $\sqrt{ }$ & & & \\
\hline Percentages of employees on $3 / 36 \mathrm{CWW}$ & $\mathrm{v}$ & & & & $\checkmark$ & $\sqrt{ }$ & $\sqrt{ }$ & & & \\
\hline Percentages of employees on $4 / 40 \mathrm{CWW}$ & $\checkmark$ & $\checkmark$ & & & & $\sqrt{ }$ & $\sqrt{ }$ & $\checkmark$ & & \\
\hline Percentages of employees on $5 / 40$ & $v$ & & & & & $\sqrt{ }$ & $\sqrt{ }$ & & & \\
\hline Percentages of employees on $7 / 40 \mathrm{CWW}$ & $\mathrm{v}$ & & & $\checkmark$ & & $\sqrt{ }$ & $\sqrt{ }$ & & & \\
\hline Percentages of employees on $9 / 80 \mathrm{CWW}$ & $\checkmark$ & $\sqrt{ }$ & & & & $\sqrt{ }$ & $\sqrt{ }$ & & & \\
\hline Percentages of employees on other CWW & $\checkmark$ & & $\sqrt{ }$ & $\sqrt{ }$ & & $\sqrt{ }$ & $\sqrt{ }$ & $\sqrt{ }$ & $\sqrt{ }$ & $\sqrt{ }$ \\
\hline Alone share & $v$ & & & $\checkmark$ & $\checkmark$ & $\sqrt{ }$ & & $\checkmark$ & & $\checkmark$ \\
\hline Bike share & $\checkmark$ & & $\checkmark$ & $\sqrt{ }$ & & $\sqrt{ }$ & $\sqrt{ }$ & $\sqrt{ }$ & & $\checkmark$ \\
\hline Bus share & $\checkmark$ & $\checkmark$ & & & $\checkmark$ & $\sqrt{ }$ & $\sqrt{ }$ & & $\sqrt{ }$ & \\
\hline Cars hare & $\checkmark$ & & $\checkmark$ & $\checkmark$ & & $\sqrt{ }$ & $\sqrt{ }$ & $\checkmark$ & & $\checkmark$ \\
\hline Other share & $v$ & $\checkmark$ & $\checkmark$ & & & $\sqrt{ }$ & $\sqrt{ }$ & & & \\
\hline Tele share & $\checkmark$ & & & & & $\sqrt{ }$ & $\sqrt{ }$ & & $\sqrt{ }$ & \\
\hline Van share & $\checkmark$ & & & & & $\sqrt{ }$ & $\sqrt{ }$ & $\checkmark$ & & $\checkmark$ \\
\hline Walk share & $\mathrm{v}$ & $\sqrt{ }$ & & & $\sqrt{ }$ & $\sqrt{ }$ & $\sqrt{ }$ & & $\sqrt{ }$ & \\
\hline Days saved telecommuting in two weeks & $\checkmark$ & & & & & - & - & - & - & - \\
\hline Administration job Share & $\checkmark$ & & & $\sqrt{ }$ & & - & - & - & - & - \\
\hline Craft/Production/Labor Share & $\mathrm{v}$ & & $\checkmark$ & & & - & - & - & - & - \\
\hline Management job Share & $\checkmark$ & & & & & - & - & - & - & - \\
\hline Sales/Marketing job Share & $\checkmark$ & & & & & - & - & - & - & - \\
\hline Customer Service job Share & $\checkmark$ & & $\checkmark$ & & & - & - & - & - & - \\
\hline Other job Share & $\checkmark$ & & $\sqrt{ }$ & & & - & - & - & - & - \\
\hline Professional/Technical job Share & $\checkmark$ & & $\sqrt{ }$ & & & - & - & - & - & - \\
\hline Q8_Invalid_Share & $\checkmark$ & & & & $\checkmark$ & - & - & - & - & - \\
\hline Prefer provide car for work & $\checkmark$ & & & & & - & - & - & - & - \\
\hline Employee Prefer Transport during lunch & $\checkmark$ & & $\checkmark$ & $\checkmark$ & & - & - & - & - & - \\
\hline Employee Prefer GRH & $\checkmark$ & & & & & - & - & - & - & - \\
\hline Employee Prefer flex to meet CVpool bus & $\checkmark$ & & & & & - & - & - & - & - \\
\hline Employee Prefer financial incentive & $\checkmark$ & & & & & - & - & - & - & - \\
\hline $\begin{array}{l}\text { Employee Prefer reserved discounted } \\
\text { CVpool space }\end{array}$ & $\checkmark$ & $\checkmark$ & & $\checkmark$ & & - & - & - & - & - \\
\hline $\begin{array}{l}\text { Employee Prefer Personalized help for } \\
\text { CVpool }\end{array}$ & $\checkmark$ & & $\sqrt{ }$ & $\sqrt{ }$ & $\sqrt{ }$ & - & - & - & - & - \\
\hline Employee Prefer covered bicycle parking & $\checkmark$ & & & & & - & - & - & - & - \\
\hline Employee Prefer lockers \& showers & $\checkmark$ & & & & & - & - & - & - & - \\
\hline Employee Prefer onsite childcare & $\mathrm{v}$ & & $\checkmark$ & & & - & - & - & - & - \\
\hline Employee Prefer CWW & $\checkmark$ & & & & $\checkmark$ & - & - & - & - & - \\
\hline Employee Prefer to telecommute & $\checkmark$ & & & & & - & - & - & - & - \\
\hline $\begin{array}{l}\text { Employee Prefer improved access to } \\
\text { transit }\end{array}$ & $\checkmark$ & & $\sqrt{ }$ & & $\sqrt{ }$ & - & - & - & - & - \\
\hline sov & $\checkmark$ & $\checkmark$ & $\checkmark$ & $\sqrt{ }$ & $\checkmark$ & - & - & - & - & - \\
\hline VMT & $\checkmark$ & $\checkmark$ & & $\sqrt{ }$ & & 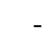 & - & - & - & - \\
\hline VTR & $\checkmark$ & & $\checkmark$ & & & $\checkmark$ & $\sqrt{ }$ & $\checkmark$ & $\sqrt{ }$ & $\checkmark$ \\
\hline Central Business District & $v$ & & & & & $\sqrt{ }$ & $\sqrt{ }$ & & & \\
\hline Suburban area & $\checkmark$ & & & & & $\sqrt{ }$ & $\sqrt{ }$ & & & \\
\hline Outside suburban area & $\checkmark$ & & & & & $\sqrt{ }$ & $\sqrt{ }$ & & & \\
\hline
\end{tabular}

From the plots in Figures 40 and 41, it is apparent that the model built using superficial variable selection obtained the best 'bin classification accuracy in moderate range of change in VTR' (22.37 percent), the best $\mathrm{R}$-square value (0.16) and the 'best accuracy on full range of change in VTR. But from Table 28 it can be seen that this model selected very few insignificant incentives that were considered as unsuitable. All neural net models built with all of the variables, selected many of the employee's preferences for incentives. This shows that the preferences did play an important role in predicting the change in VTR. As stated earlier, the 
employers might not be able to have access to this employee information; and so these models will be used simply for study.

Considering all of the performance measures and the variables included, the linear forced enter regression model (M1) and neural network model (M2) built on data without preference variables can be considered suitable. The accuracies for these models on each of the bins are shown in Table 29.

Table 27: Detailed accuracies on bins

\begin{tabular}{|c|c|c|c|c|c|c|c|c|c|c|c|c|}
\hline & \multirow[b]{3}{*}{ Range } & \multirow{3}{*}{$\begin{array}{c}\text { \# of } \\
\text { records }\end{array}$} & \multirow{2}{*}{\multicolumn{8}{|c|}{ Bin ranges over Change in Vehicle Trip Rate (Delta_VTR) }} & \multirow{7}{*}{$\begin{array}{l}\text { Weighted } \\
\text { Avg on } \\
\text { a2 to a5 }\end{array}$} & \multirow{6}{*}{ R-square } \\
\hline & & & & & & & & & & & & \\
\hline & & & $>-7$ & \begin{tabular}{|c|}
{$[-7$ to -} \\
$4.5)$
\end{tabular} & $\begin{array}{c}{[-4.5 \text { to }} \\
-3)\end{array}$ & $\begin{array}{c}{[-3 \text { to }-} \\
1.5)\end{array}$ & $\begin{array}{c}{[-1.5 \text { to }} \\
0)\end{array}$ & $\begin{array}{l}\text { [0 to } \\
1.5)\end{array}$ & $\begin{array}{c}\text { [1.5 to } \\
3.5)\end{array}$ & $3.5>=$ & & \\
\hline & Bin Number & & a1 & a2 & a3 & a4 & A5 & a6 & A7 & a8 & & \\
\hline & Validation & 144 & 15 & 15 & 15 & 17 & 20 & 20 & 21 & 21 & & \\
\hline & Training & 1300 & 136 & 132 & 132 & 152 & 182 & 184 & 190 & 192 & & \\
\hline & & & & & & & & & & & & \\
\hline M1 & Exact Validation & $15.97 \%$ & $5.88 \%$ & $11.76 \%$ & $8.33 \%$ & $25.00 \%$ & $31.25 \%$ & $23.08 \%$ & $5.56 \%$ & $13.64 \%$ & $19.40 \%$ & 0.047 \\
\hline & Exact Training & $14.38 \%$ & $2.84 \%$ & $5.00 \%$ & $11.27 \%$ & $34.00 \%$ & $27.27 \%$ & $18.89 \%$ & $10.00 \%$ & $6.13 \%$ & $19.93 \%$ & 0.135 \\
\hline & One-off Validation & $38.89 \%$ & $17.65 \%$ & $23.53 \%$ & $25.00 \%$ & $75.00 \%$ & $75.00 \%$ & $53.85 \%$ & $11.11 \%$ & $27.27 \%$ & $50.00 \%$ & \\
\hline & One-Off Training & $46.08 \%$ & $9.93 \%$ & $28.57 \%$ & $52.82 \%$ & $80.67 \%$ & $80.61 \%$ & $59.44 \%$ & $34.12 \%$ & $24.06 \%$ & $19.40 \%$ & 0.047 \\
\hline M2 & Exact Validation & $13.19 \%$ & $0.00 \%$ & $11.76 \%$ & $16.67 \%$ & $31.25 \%$ & $18.75 \%$ & $15.38 \%$ & $5.56 \%$ & $9.09 \%$ & $19.43 \%$ & 0.063 \\
\hline & Exact Training & $21.92 \%$ & $6.38 \%$ & $14.29 \%$ & $18.31 \%$ & $32.00 \%$ & $27.27 \%$ & $25.00 \%$ & $17.06 \%$ & $29.72 \%$ & $23.28 \%$ & 0.281 \\
\hline & One-off Validation & $38.89 \%$ & $17.65 \%$ & $35.29 \%$ & $33.33 \%$ & $62.50 \%$ & $43.75 \%$ & $61.54 \%$ & $27.78 \%$ & $22.73 \%$ & $43.78 \%$ & \\
\hline & One-Off Training & $53.38 \%$ & $26.95 \%$ & $37.14 \%$ & $50.70 \%$ & $66.00 \%$ & $63.64 \%$ & $66.67 \%$ & $48.24 \%$ & $59.43 \%$ & & \\
\hline
\end{tabular}

The scatter plots for the validation set for these models are shown in Figures 43 and 44 .

Actual Delta_VTR Vs. Predicted Delta_VTR

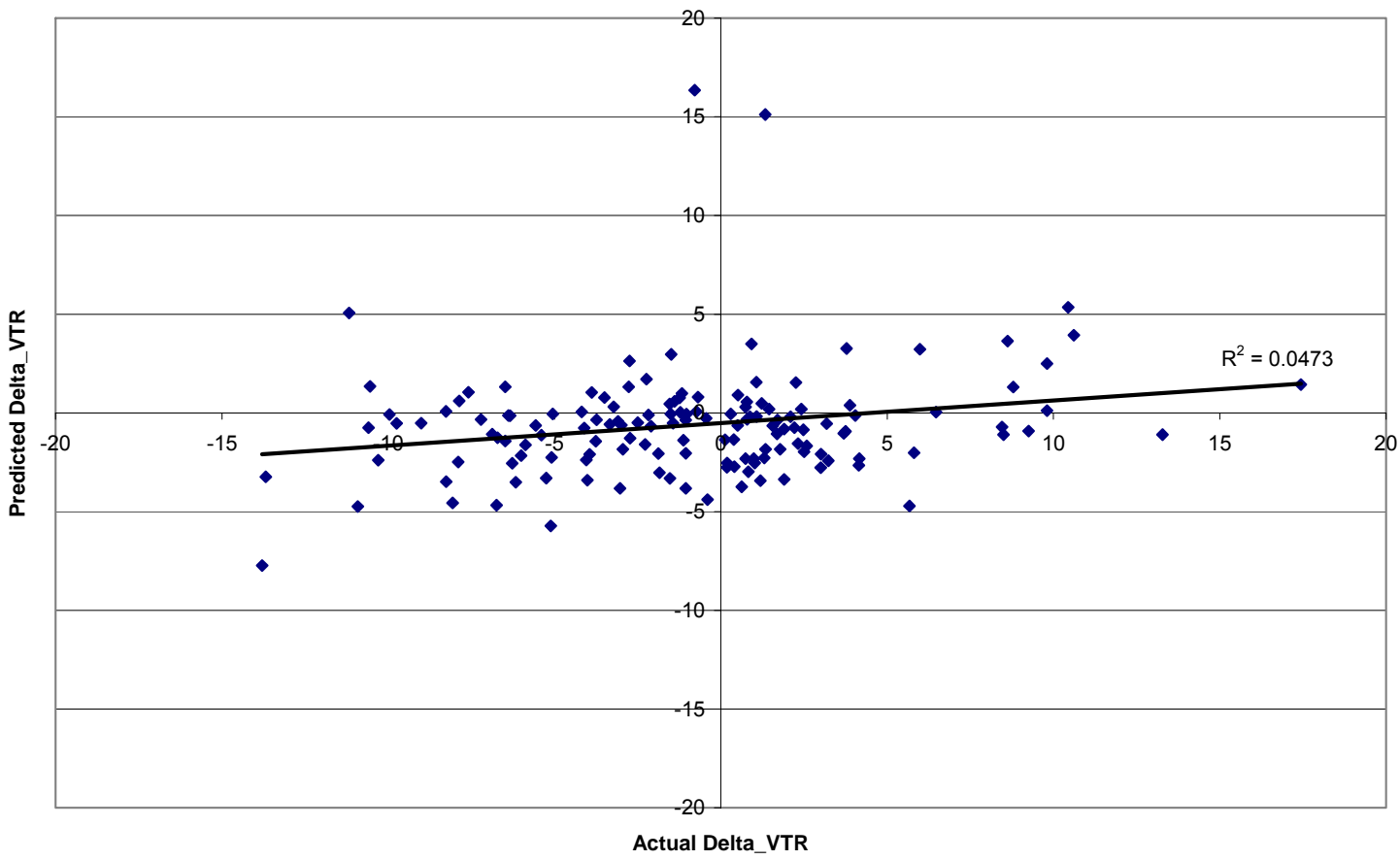

Figure 43: Scatter plot for linear forced enter regression model (M1) 
Actual Delta_VTR Vs. Predicted Delta_VTR

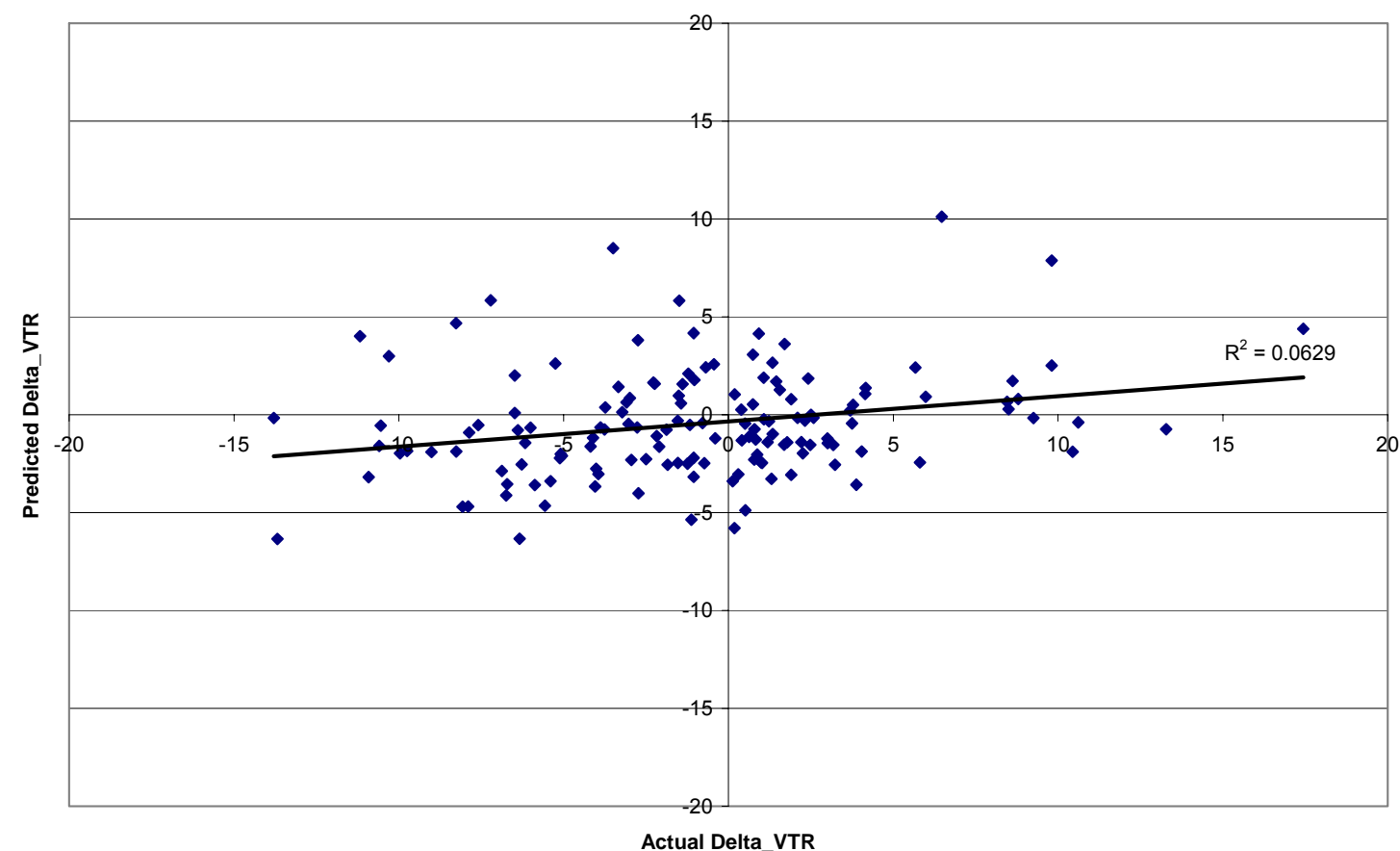

Figure 44: Scatter plot for neural network model (M2)

The regression coefficients for the forced enter regression model (M1) are shown in Table 30.

Table 28: Variables and coefficients for forced enter regression model

\begin{tabular}{|l|r|r|}
\hline \multicolumn{1}{|c|}{ Variable } & coefficient & \multicolumn{1}{c|}{ t - value } \\
\hline Constant & 49.063 & 2.267 \\
Health organization & -0.386 & -0.419 \\
Retail organization & -1.695 & -1.222 \\
Manufacturing organization & 1.337 & 1.637 \\
Other organization & 0.514 & 0.965 \\
Shifts & 0.547 & 1.463 \\
Onsite Parking Spaces & -0.129 & -0.672 \\
Offsite Parking Spaces & -0.993 & -1.794 \\
Leased Onsite Parking Price & -0.002 & -0.508 \\
Leased Offsite Parking Price & -0.006 & -1.130 \\
Own Onsite Parking & 0.956 & 2.412 \\
Onsite Parking Charge & -0.004 & -0.327 \\
Own Offsite Parking & -0.254 & -0.395 \\
Offsite Parking Charge & 0.010 & 1.103 \\
Pay Parking Charge & 0.007 & 1.275 \\
on off parking sub & -1.793 & -2.587 \\
Free Parking 1/4 mile & 0.321 & 1.142 \\
ETC Additional Training & 0.170 & 0.507 \\
ETC Onsite & 0.040 & 0.088 \\
Distribute Info & 0.005 & 1.253 \\
Post Materials & 0.001 & 0.121 \\
CTR Orientation & 0.000 & 0.665 \\
CTR Events & -0.125 & -2.366 \\
CTR E-mail & -0.005 & -0.390 \\
Articles & 0.017 & 0.486 \\
Ridematch Apps & -0.002 & -0.678
\end{tabular}




\begin{tabular}{|l|r|r|}
\hline \multicolumn{1}{|c|}{ Variable } & coefficient & t - value \\
\hline With Paychecks & 0.038 & 0.899 \\
Drawings & 0.071 & 2.147 \\
Other Promo & 0.004 & 0.664 \\
Covered Bike Number & 0.183 & 0.155 \\
Uncovered Bike Number & 3.276 & 1.024 \\
Lockers Number & -0.018 & -0.077 \\
Showers Number & -7.013 & -1.348 \\
Shelters Number & -2.191 & -0.411 \\
Other Amenities 1 Number & -24.724 & -3.366 \\
Carpool Spaces Number & -1.593 & -1.556 \\
Vanpool Spaces Number & 4.003 & 1.121 \\
SOV Parking Charge & -0.012 & -1.935 \\
SOV Parking Charge Number & 0.693 & 1.698 \\
Reduced SOVP Number & 2.695 & 1.217 \\
Transit Subsidy & -0.002 & -0.349 \\
Ferry Subsidy & 0.021 & 1.575 \\
Vanpool Subsidy & -0.024 & -1.770 \\
Carpool Subsidy & 0.007 & 0.555 \\
Walking Subsidy & 0.023 & 0.673 \\
Bike Subsidy & -0.006 & -0.322 \\
employees on flextime & 0.518 & 0.700 \\
employees with GRH & 0.241 & 0.655 \\
employees in-house match & -0.200 & -1.209 \\
employees public match & -0.126 & -1.257 \\
FV work employees & -0.013 & -0.006 \\
Number of Employee & -3.150 & -0.577 \\
Percentages of employees on 3/36 CWW & -2.601 & -0.525 \\
Percentages of employees on 4/40 CWW & -2.070 & -0.449 \\
\hline Percentages of employees on 5/40 & & \\
Percentages of employees on 7/40 CWW & -0.621 & -0.056 \\
Percentages of employees on 9/80 CWW & -4.976 & -1.085 \\
Percentages of employees on other CWW & -1.665 & -0.675 \\
Bike share & -6.289 & -0.843 \\
Bus share & -4.998 & -1.562 \\
Cars hare & -18.379 & -2.609 \\
Other share & -18.862 & -0.856 \\
Tele share & -45.089 & -2.274 \\
Van share & -14.707 & -1.280 \\
Walk share & -30.923 & -1.372 \\
VTR & -47.565 & -1.744 \\
Central Business District & -20.061 & -0.844 \\
Suburban area & -2.490 \\
Outside suburban area & -286 \\
\hline
\end{tabular}


To reduce to complexity of the models, all of the individual incentives were replaced with the grouped incentives and simple neural network and regression model with no variable selection were built on the data just containing the grouped incentives and worksite characteristics like modesplits. The variables for the grouped incentives and worksite characteristics are shown in Table 31.

Table 29: Variables with grouped incentives

\begin{tabular}{|c|c|}
\hline Variables & Description \\
\hline Health & Health organization \\
\hline Retail & Retail organization \\
\hline Manufact & Manufacturing organization \\
\hline other new & other organization \\
\hline Shifts & Shifts \\
\hline FACILITY_AMENITIES & $\begin{array}{l}\text { Covered/uncovered bike parking, lockers a\& showers, shelters, carpool \& vanpool spaces, } \\
\text { other amenities }\end{array}$ \\
\hline $\mathrm{GRH}$ & Guaranteed ride home program \\
\hline FLEX & flexible timing \\
\hline Mrkt & CTR Events, CTR E-mail, Articles, With Paychecks, Other Promo, etc \\
\hline RS_MATCH & Ride match Apps, employees in-house match, employees public match \\
\hline FINANCIAL & Ferry, Carpool, Walking, Bike Subsidy \\
\hline PARKMGT & SOV Parking Charge, SOV Parking Charge Number, Reduced SOVP Number \\
\hline CWW & compressed work week $3 / 36,4 / 40,9 / 80,7 / 40$, other \\
\hline onsite & onsite incentives \\
\hline direct_nonfinan & Drawing, leaves, etc \\
\hline commtax & Transit, vanpool subsidy \\
\hline EmpNo & Number of employees \\
\hline CWW3 & Percentages of employees on $3 / 36 \mathrm{CWW}$ \\
\hline CWW4 & Percentages of employees on $4 / 40 \mathrm{CWW}$ \\
\hline CWW5 & Percentages of employees on $5 / 40$ \\
\hline CWW7 & Percentages of employees on $7 / 40 \mathrm{CWW}$ \\
\hline CWW9 & Percentages of employees on $9 / 80 \mathrm{CWW}$ \\
\hline CWWOTHER & Percentages of employees on other CWW \\
\hline AloneShr & Alone share \\
\hline BikeShr & Bike share \\
\hline BusShr & Bus share \\
\hline CarShr & Cars hare \\
\hline OtherShr & Other share \\
\hline TeleShr & Tele share \\
\hline VanShr & Van share \\
\hline WalkShr & Walk share \\
\hline VTR & VTR \\
\hline CBD & Central Business District \\
\hline Suburb & Suburban area \\
\hline Outside & Outside suburban area \\
\hline
\end{tabular}

Figures 45, 46, and 47 show the comparison of the different performance measures on this grouped incentive data. 


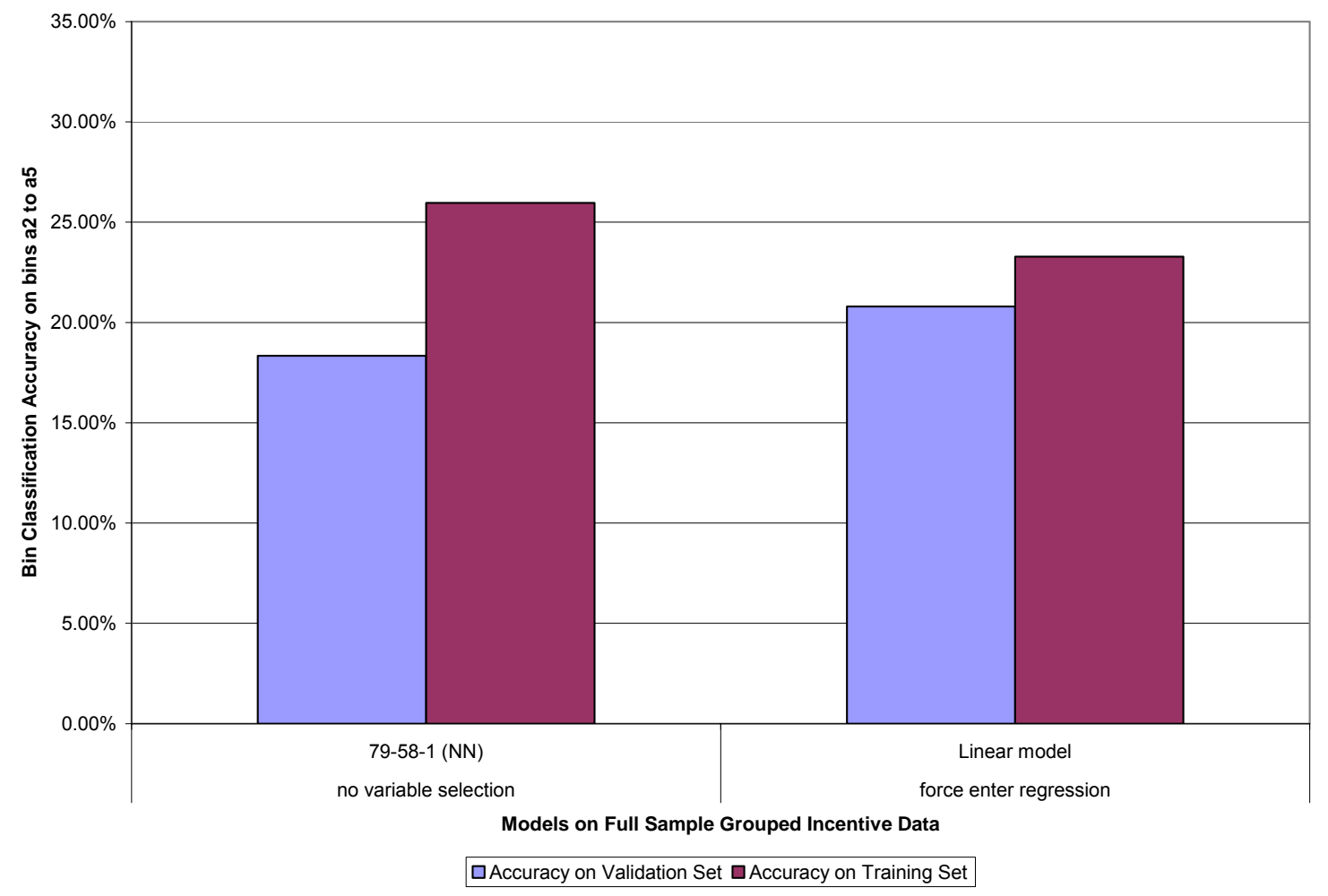

Figure 45: Bin Classification Accuracy on Moderate Range of change in VTR (a2 to a5) for validation \& training set (Different models on Full sample Grouped Incentive data)

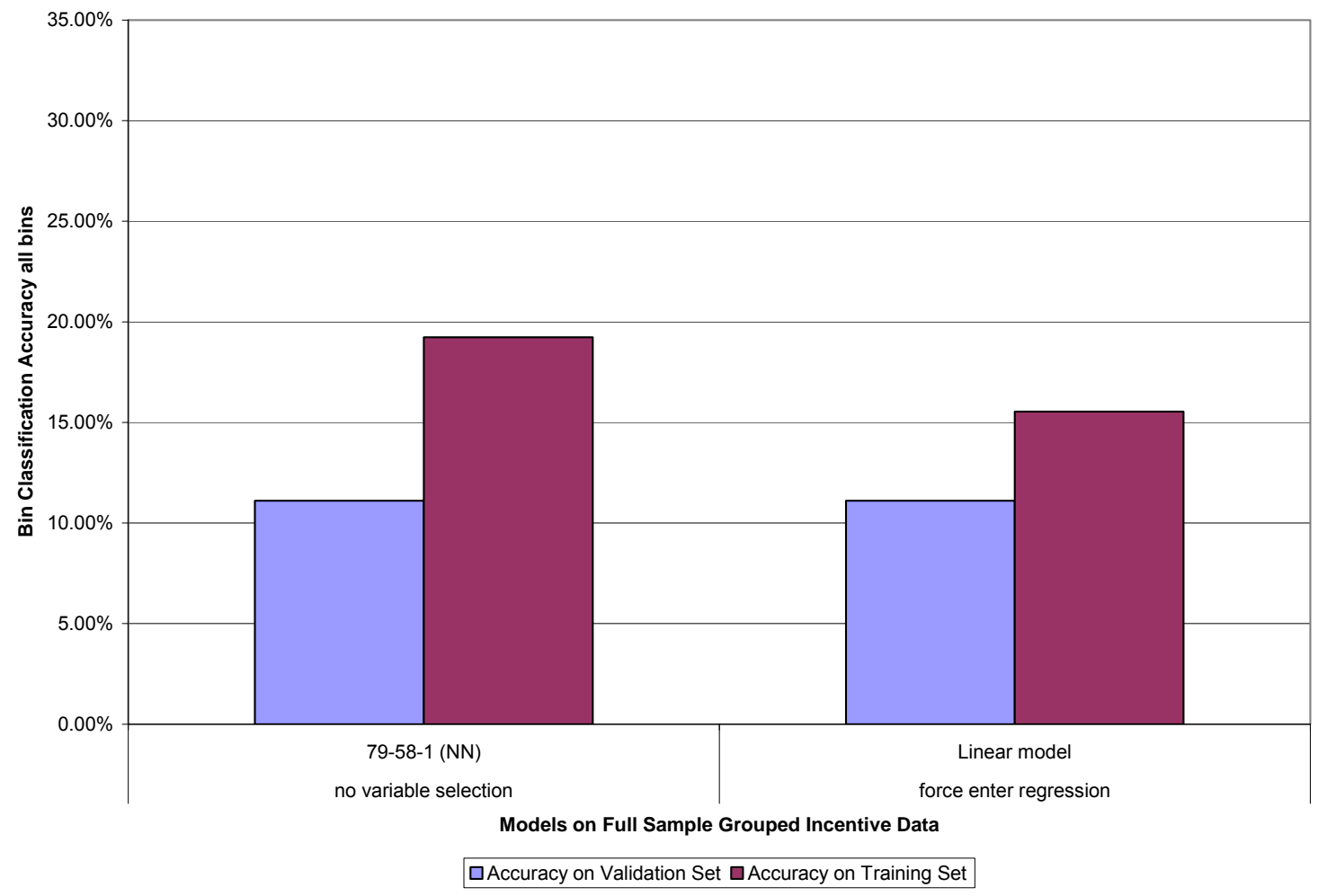

Figure 46: Bin Classification Accuracy on Full Range of change in VTR (all bins) for validation \& training set (Different models on Full sample Grouped Incentive data) 


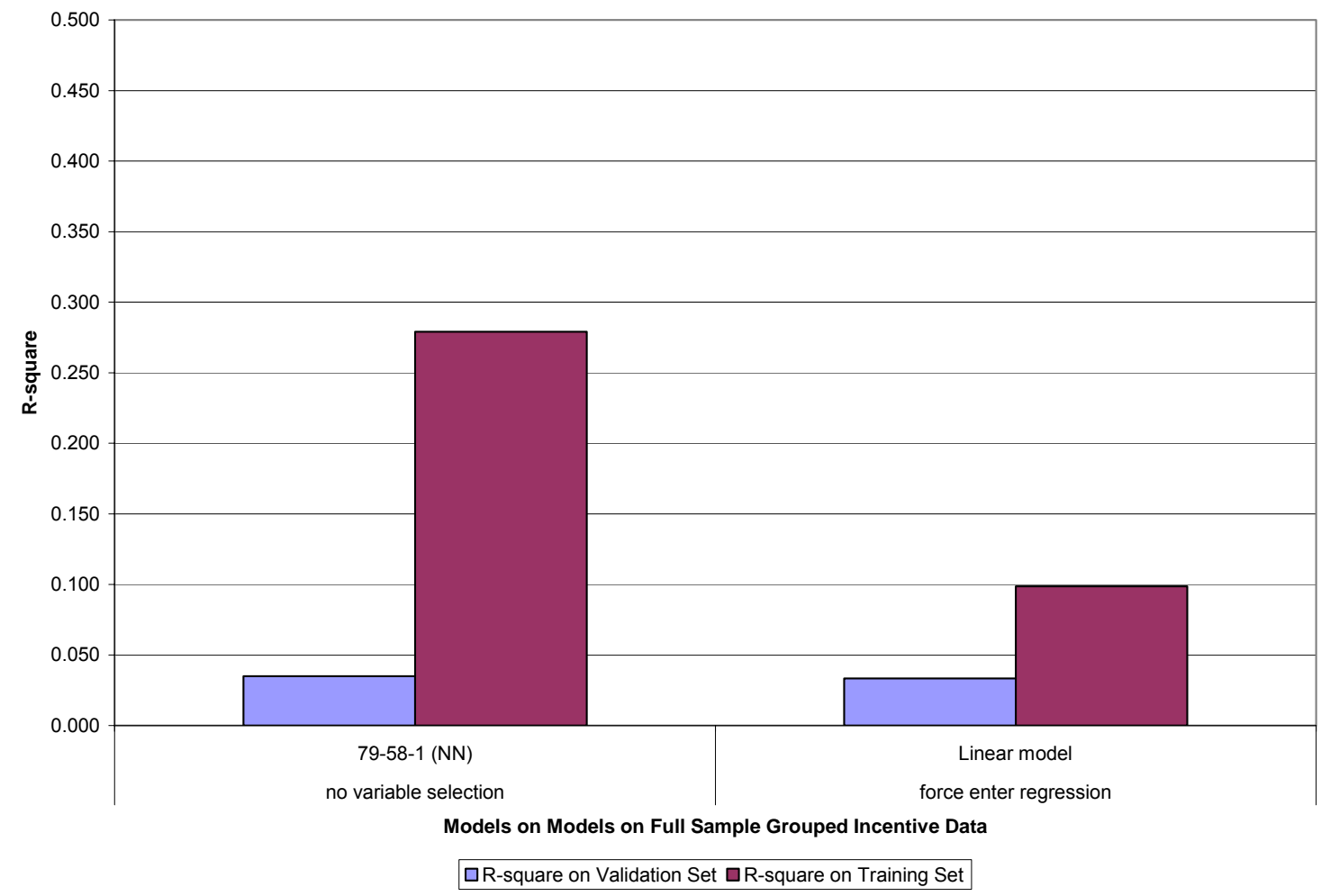

Figure 47: R-square for validation \& training set (Different models on Full sample Grouped Incentive data)

The charts in Figures 45, 46, and 47 show that both of the models, neural network (M1) and linear forced enter regression (M2), are comparable to each other for 'bin classification accuracy on full range of change in VTR' but reduced as compared to models built on earlier ungrouped data. Even though the new linear forced enter regression model that was built on grouped incentives was better distributed than the previous model built with ungrouped incentives, the linear forced enter regression (M2) model was still not as well distributed between the bins as the neural network (M1) model. The detailed accuracies for both the models are shown in Table 32.

Table 30: Detailed accuracies on bins

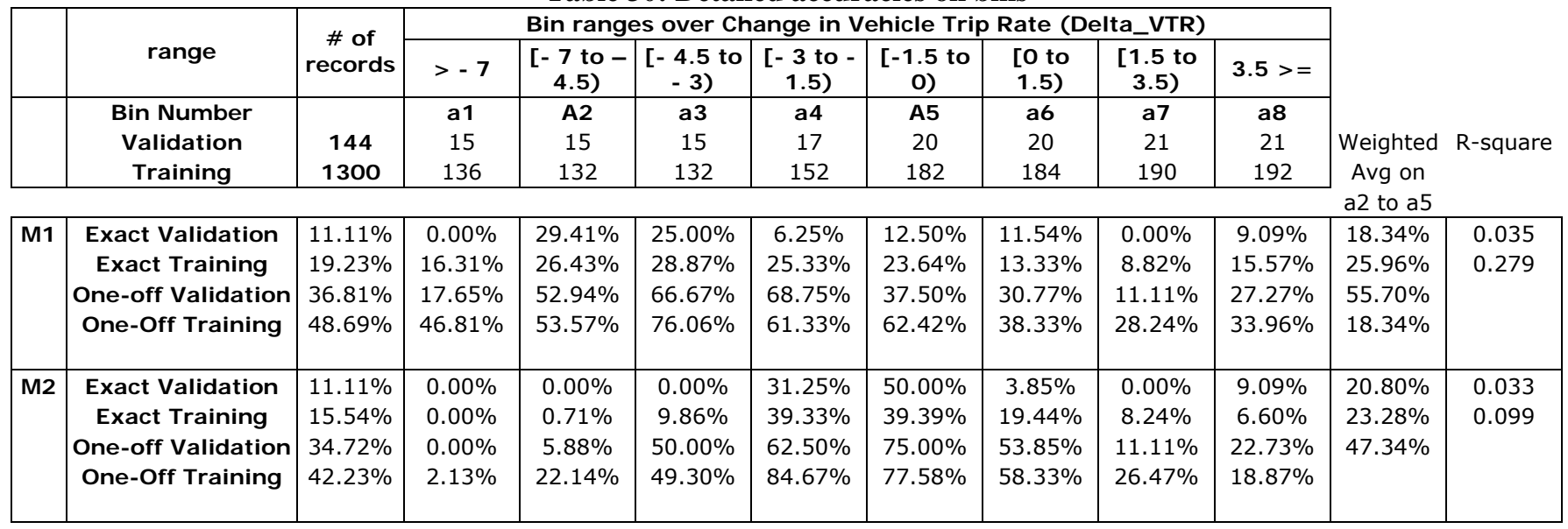


The scatter plots for the validation set of the forced enter regression model and the neural net model with no variable selection are shown in Figures 48 and 49.

Actual Delta_VTR Vs. Predicted Delta_VTR

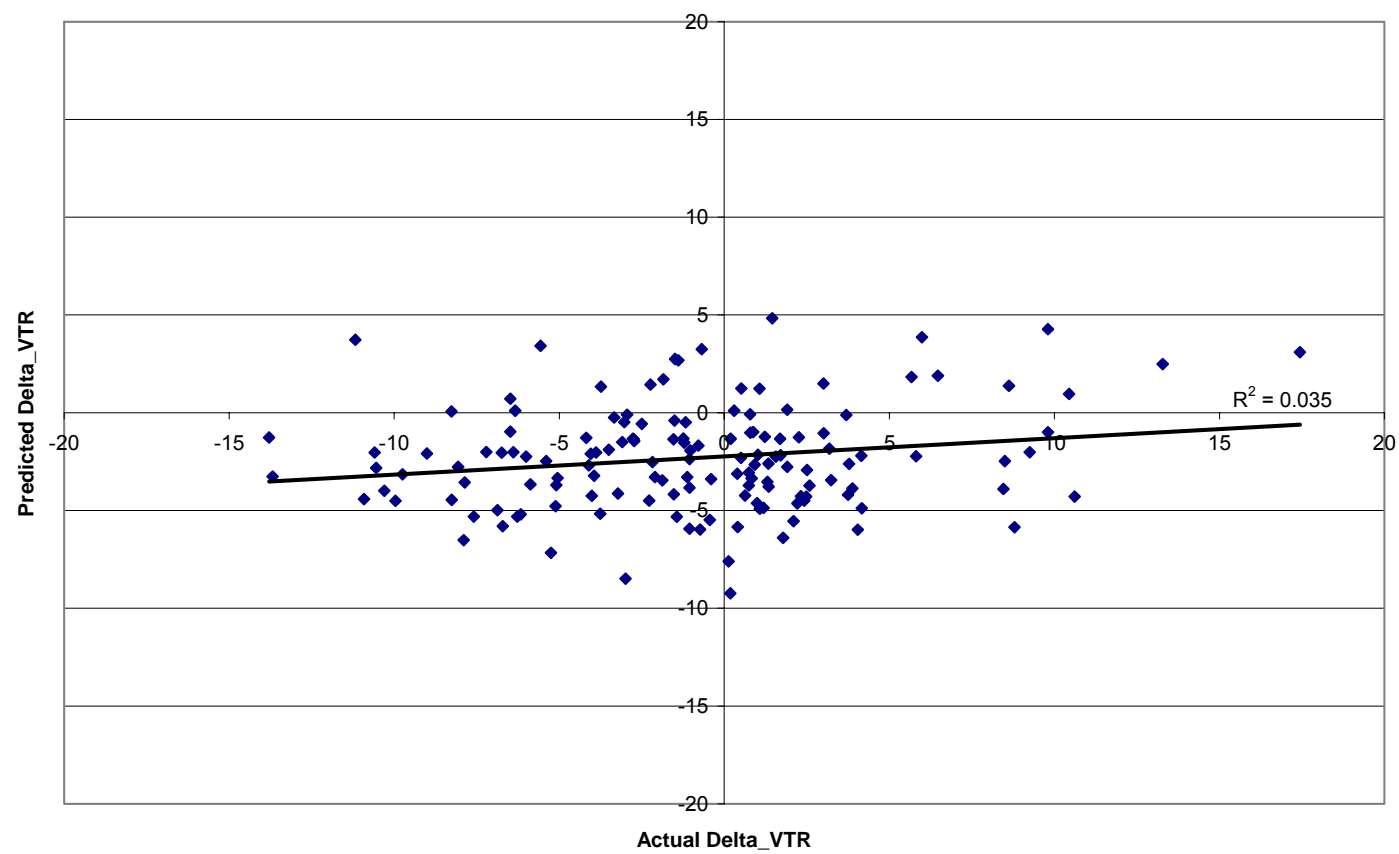

Figure 48: Scatter plot for neural network model (M1)

Actual Delta_VTR Vs. Predicted Delta_VTR

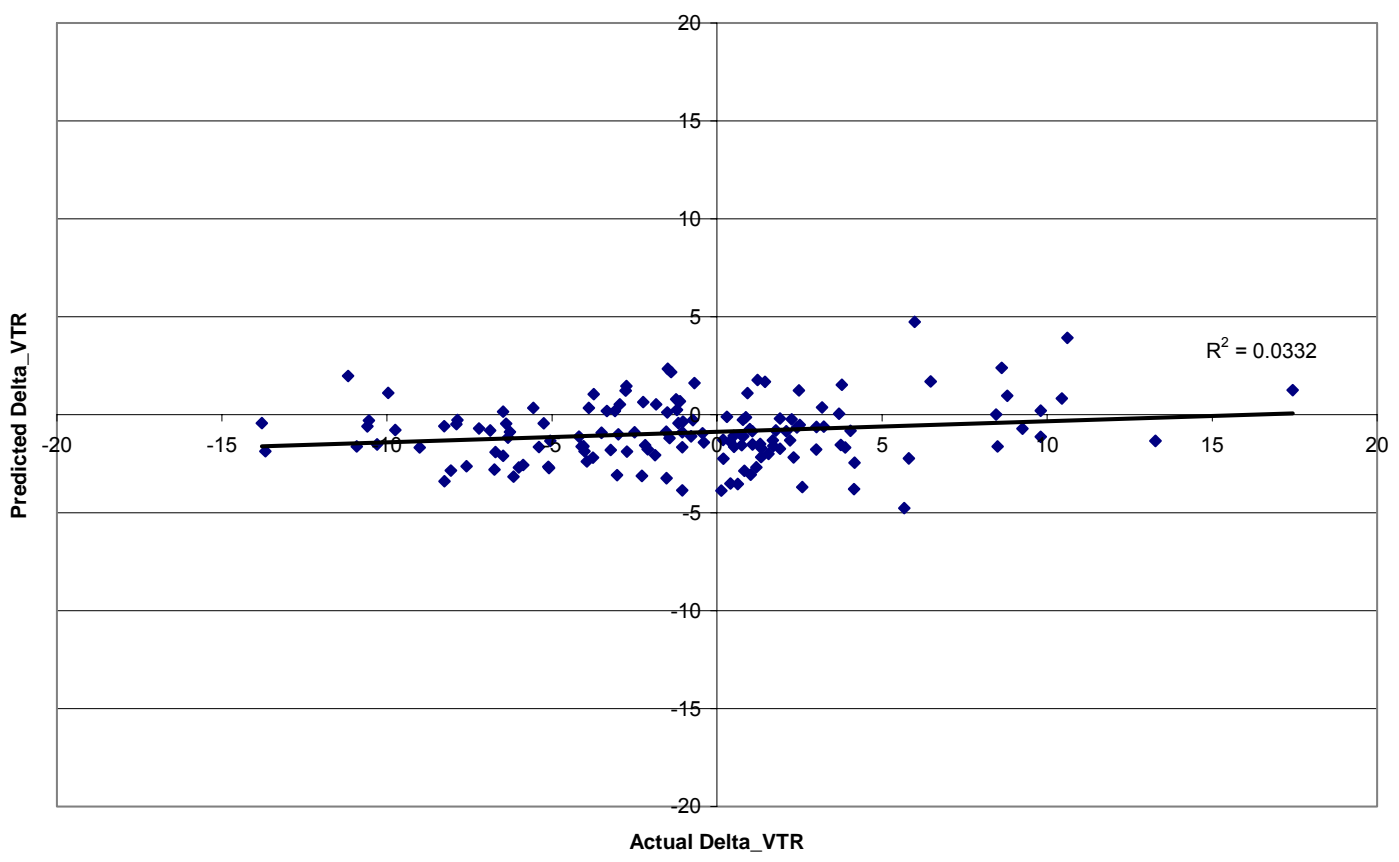

Figure 49: Scatter plot for linear forced enter regression model (M2) 
The regression coefficients for the linear forced enter regression model (M2) (grouped incentives) are shown in table 33.

Table 31: Variables and coefficients for forced enter regression model

\begin{tabular}{|c|c|c|}
\hline Variables & coefficients & t - value \\
\hline Constant & 42.042 & 2.015 \\
\hline Health organization & -0.177 & -0.192 \\
\hline Retail organization & -1.915 & -1.398 \\
\hline Manufacturing organization & 0.817 & 1.157 \\
\hline other organization & 0.099 & 0.262 \\
\hline Shifts & 0.735 & 2.106 \\
\hline $\begin{array}{l}\text { covered/uncovered bike parking, lockers a\& showers, shelters, carpool \& vanpool } \\
\text { spaces, other amenities }\end{array}$ & -0.825 & -1.608 \\
\hline Guaranteed ride home program & 0.620 & 1.597 \\
\hline flexible timing & 0.267 & 0.745 \\
\hline CTR Events, CTR E-mail, Articles, With Paychecks, Other Promo, etc & -0.093 & -0.945 \\
\hline Ride match Apps, employees in-house match, employees public match & -0.303 & -0.910 \\
\hline Ferry, Carpool, Walking, Bike Subsidy & 0.282 & 0.685 \\
\hline SOV Parking Charge, SOV Parking Charge Number, Reduced SOVP Number & -0.346 & -1.038 \\
\hline compressed work week $3 / 36,4 / 40,9 / 80,7 / 40$, other & -0.577 & -0.733 \\
\hline Onsite incentives & 0.584 & 1.315 \\
\hline Drawing, leaves, etc & 1.045 & 2.578 \\
\hline Transit, vanpool subsidy & -0.101 & -0.271 \\
\hline Number of employees & 0.000 & -0.012 \\
\hline Percentages of employees on $3 / 36 \mathrm{CWW}$ & 5.214 & 0.420 \\
\hline Percentages of employees on $4 / 40 \mathrm{CWW}$ & -1.812 & -0.418 \\
\hline Percentages of employees on $5 / 40$ & 0.318 & 0.158 \\
\hline Percentages of employees on $7 / 40 \mathrm{CWW}$ & -3.156 & -0.394 \\
\hline Percentages of employees on $9 / 80 \mathrm{CWW}$ & -1.915 & -0.602 \\
\hline Percentages of employees on other CWW & -13.141 & -1.944 \\
\hline Bike share & -14.208 & -0.671 \\
\hline Bus share & -40.968 & -2.042 \\
\hline Cars hare & -11.967 & -1.050 \\
\hline Other share & -22.955 & -0.983 \\
\hline Tele share & -53.781 & -2.064 \\
\hline Van share & -15.433 & -0.689 \\
\hline Walk share & -43.659 & -2.108 \\
\hline VTR & -0.449 & -2.198 \\
\hline Central Business District & -2.240 & -0.567 \\
\hline Suburban area & -1.297 & -0.331 \\
\hline Outside suburban area & -0.870 & -0.223 \\
\hline
\end{tabular}




\section{Phase II: Washington Over-Sampled Data}

To get better accuracies on moderate range bins a 2 to a5, the examples in these bins were over-sampled. The Table 34 shows the changes in the number of examples in the training set due to over-sampling.

Table 32: Washington Over-Sampled Data - No. of Records in bins

\begin{tabular}{|c|c|c|c|c|c|c|c|c|c|}
\hline \multirow{2}{*}{ Range } & \multirow[b]{2}{*}{ \# of records } & \multicolumn{8}{|c|}{ Bin ranges over Change in Vehicle Trip Rate (Delta_VTR) } \\
\hline & & $>-7$ & [- 7 to -4.5$)$ & {$[-4.5$ to -3$]$} & [- 3 to -1.5$)$ & {$[-1.5$ to 0$)$} & [0 to 1.5) & [1.5 to 3.5$)$ & $3.5>=$ \\
\hline Bin Number & & a1 & a2 & A3 & a4 & a5 & a6 & a7 & a8 \\
\hline Validation & 144 & 15 & 15 & 15 & 17 & 20 & 20 & 21 & 21 \\
\hline Training & 1862 & 150 & 172 & 282 & 341 & 306 & 214 & 178 & 219 \\
\hline
\end{tabular}

Neural network models were built of data containing the entire variable set (105 variables) with different variable selections. As discussed in the previous phase, the dataset contained variables such as shares of employee's incentive preference and other information that employers might not be able to provide to about their employees. These variables with a few other insignificant variables were removed from the data.

Then a neural network model with no variable selection and all different types of regression models were built on data without the preference variables. Figures 50, 51, and 52 show the different performance measure for all these models.

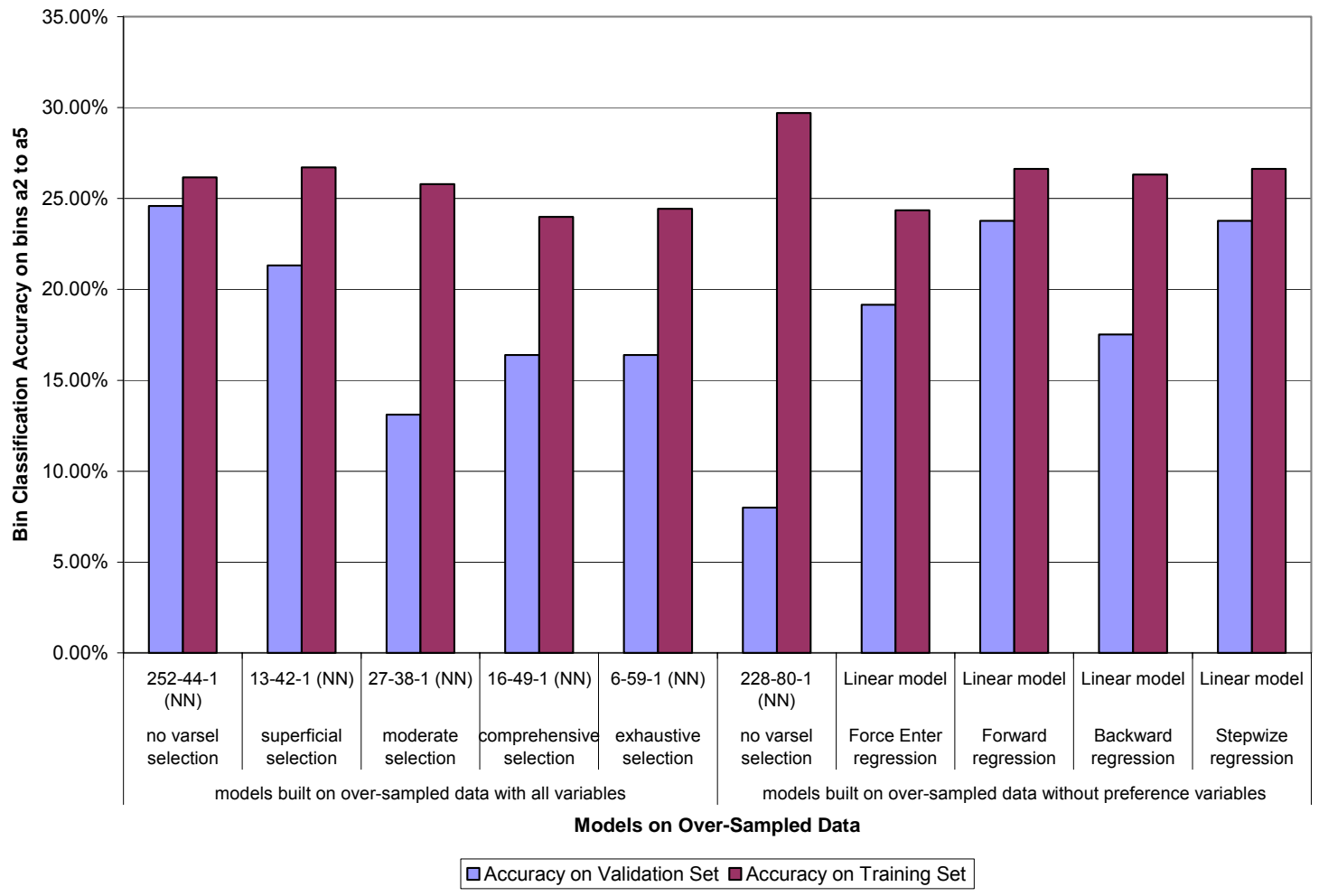

Figure 50: Bin Classification Accuracy on Moderate Range of change in VTR (a2 to a5) for validation \& training set (Different models on over-sampled data) 


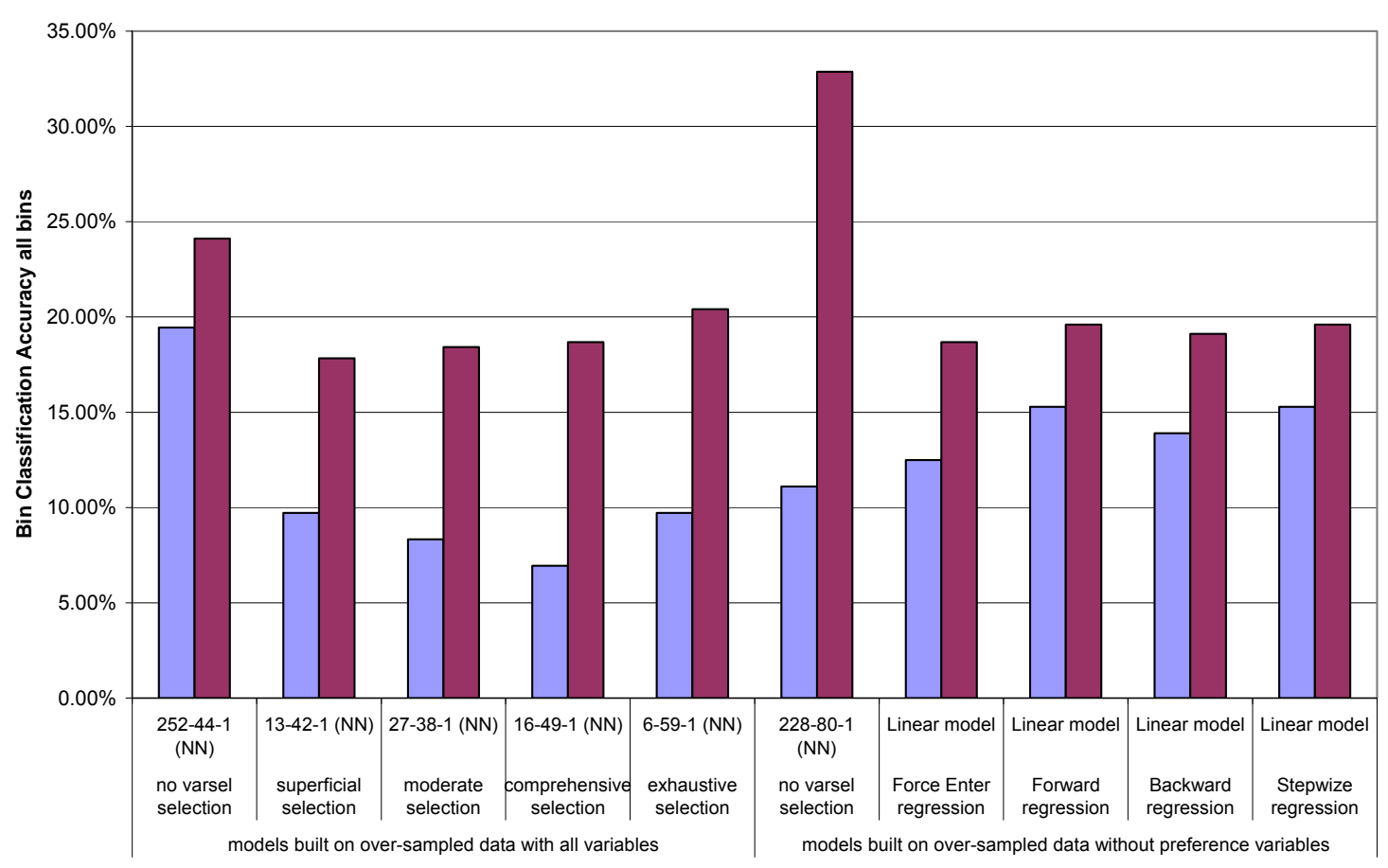

Models on Over-Sampled Data

$\square$ Accuracy on Validation Set $\square$ Accuracy on Training Set

Figure 51: Bin Classification Accuracy on Full Range of change in VTR (all bins) for validation \& training set (Different models on over-sampled data)

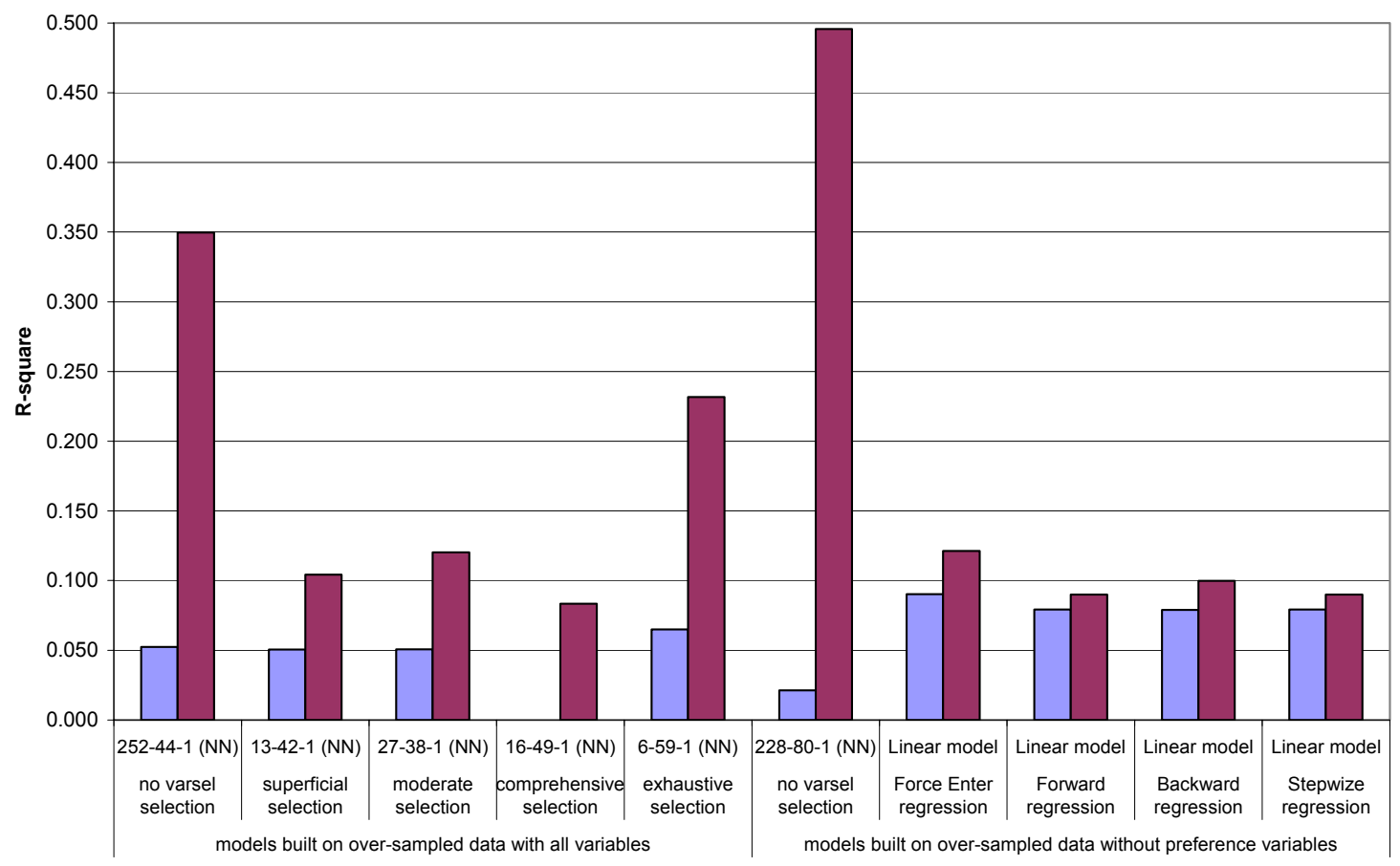

Models on Over-Sampled Data

$\square R$-square on Validation Set $\square$ R-square on Training Set

Figure 52: R-square for validation \& training set (Different models on over-sampled data) 
Table 35 shows the variables selected using the without preferences datasets to build models.

Column name abbreviations,

$\mathrm{N}$ - Neural network model without variable selection

$S$ - Neural network model with superficial variable selection

M - Neural network model with moderate variable selection

C - Neural network model with comprehensive variable selection

E - Neural network model with exhaustive variable selection

FE - Linear Forced Enter regression model

FR - Linear Forward regression model

BK - Linear Backward regression model

SP - Linear Stepwise regression model

$\checkmark$ - indicates the selection of the variable

'-' - indicates the variable was not present in the data

Table 33: Variables selected by different models on over-sampled data

\begin{tabular}{|c|c|c|c|c|c|c|c|c|c|c|}
\hline \multirow[t]{2}{*}{ Variables } & \multicolumn{5}{|c|}{ NN models on data with all variables } & \multicolumn{5}{|c|}{$\begin{array}{l}\text { Models on data without preference } \\
\text { and other insignificant variables }\end{array}$} \\
\hline & $\mathbf{N}$ & $\mathbf{S}$ & $\mathbf{M}$ & C & $\mathbf{E}$ & $\mathbf{N}$ & FE & FR & BK & SP \\
\hline Non-profit organization & $\sqrt{ }$ & & & & & - & - & - & - & - \\
\hline Agriculture organization & & & & & & - & - & - & - & - \\
\hline Finance organization & $\sqrt{ }$ & & & & & - & - & - & - & - \\
\hline Info services organization & $\sqrt{ }$ & & $\sqrt{ }$ & & & - & - & - & - & - \\
\hline Health organization & $\sqrt{ }$ & & & & & $\sqrt{ }$ & $\sqrt{ }$ & & & \\
\hline Retail organization & $\sqrt{ }$ & & & & & $\sqrt{ }$ & $\sqrt{ }$ & & & \\
\hline Manufacturing organization & $\sqrt{ }$ & & & & & $\sqrt{ }$ & $\sqrt{ }$ & & $\sqrt{ }$ & \\
\hline Services organization & $\sqrt{ }$ & & $\sqrt{ }$ & & & - & - & - & - & - \\
\hline Public utilities organization & $\sqrt{ }$ & & & & & - & - & - & - & - \\
\hline Construction organization & & & & & & - & - & - & - & - \\
\hline Transportation organization & $\sqrt{ }$ & & & & & - & - & - & - & - \\
\hline Government organization & $\sqrt{ }$ & & & & & - & - & - & - & - \\
\hline Other organization & $\sqrt{ }$ & & & & & $\sqrt{ }$ & $\sqrt{ }$ & & $\sqrt{ }$ & \\
\hline Offered to All & $\sqrt{ }$ & & & & & - & - & - & - & - \\
\hline Union & $\sqrt{ }$ & & & & & $\sqrt{ }$ & $\sqrt{ }$ & & & \\
\hline Shifts & $\sqrt{ }$ & & $\sqrt{ }$ & & & $\sqrt{ }$ & $\sqrt{ }$ & & $\sqrt{ }$ & \\
\hline Onsite Parking Spaces & $\sqrt{ }$ & & & $\sqrt{ }$ & & $\sqrt{ }$ & $\sqrt{ }$ & & & \\
\hline Offsite Parking Spaces & $\sqrt{ }$ & & $\sqrt{ }$ & & & $\sqrt{ }$ & $\sqrt{ }$ & & $\sqrt{ }$ & \\
\hline Leased Onsite Parking Price & $\sqrt{ }$ & & & & & $\sqrt{ }$ & $\sqrt{ }$ & & & \\
\hline Leased Offsite Parking Price & $\sqrt{ }$ & & & $\sqrt{ }$ & & - & - & - & - & - \\
\hline Own Onsite Parking & $\sqrt{ }$ & & & $\sqrt{ }$ & $\sqrt{ }$ & $\sqrt{ }$ & $\sqrt{ }$ & $\sqrt{ }$ & $\sqrt{ }$ & $\sqrt{ }$ \\
\hline Onsite Parking Charge & $\sqrt{ }$ & & & & & $\sqrt{ }$ & $\sqrt{ }$ & & & \\
\hline Own Offsite Parking & $\sqrt{ }$ & & & & & $\sqrt{ }$ & $\sqrt{ }$ & & & \\
\hline Offsite Parking Charge & $\sqrt{ }$ & & & & & $\sqrt{ }$ & $\sqrt{ }$ & & & \\
\hline Pay Parking Charge & $\sqrt{ }$ & & & & & $\sqrt{ }$ & $\sqrt{ }$ & & & \\
\hline On off parking sub & $\sqrt{ }$ & & $\sqrt{ }$ & & & $\sqrt{ }$ & $\sqrt{ }$ & $\sqrt{ }$ & $\sqrt{ }$ & $\sqrt{ }$ \\
\hline Free Parking $1 / 4$ mile & $\sqrt{ }$ & & & & & $\sqrt{ }$ & $\sqrt{ }$ & & $\sqrt{ }$ & \\
\hline ETC Additional Training & $\sqrt{ }$ & & & & & $\sqrt{ }$ & $\sqrt{ }$ & & & \\
\hline ETC Onsite & $\sqrt{ }$ & & & & & $\sqrt{ }$ & $\sqrt{ }$ & & & \\
\hline Distribute Info & $\sqrt{ }$ & & & & & $\sqrt{ }$ & $\sqrt{ }$ & & & \\
\hline Post Materials & $\sqrt{ }$ & & & & & $\sqrt{ }$ & $\sqrt{ }$ & & & \\
\hline CTR Orientation & $\sqrt{ }$ & & $\sqrt{ }$ & & & $\sqrt{ }$ & $\sqrt{ }$ & & & \\
\hline CTR Events & $\sqrt{ }$ & & & & & $\sqrt{ }$ & $\sqrt{ }$ & $\sqrt{ }$ & $\sqrt{ }$ & $\sqrt{ }$ \\
\hline CTR E-mail & $\sqrt{ }$ & & & $\sqrt{ }$ & & $\sqrt{ }$ & $\sqrt{ }$ & & & \\
\hline Articles & $\sqrt{ }$ & & $\sqrt{ }$ & & $\sqrt{ }$ & $\sqrt{ }$ & $\sqrt{ }$ & & & \\
\hline Ride match Apps & $\sqrt{ }$ & & $\sqrt{ }$ & & & $\sqrt{ }$ & $\sqrt{ }$ & & & \\
\hline With Paychecks & $\sqrt{ }$ & & & & & $\sqrt{ }$ & $\sqrt{ }$ & & & \\
\hline Drawings & $\sqrt{ }$ & & $\sqrt{ }$ & $\sqrt{ }$ & $\sqrt{ }$ & $\sqrt{ }$ & $\sqrt{ }$ & $\sqrt{ }$ & $\sqrt{ }$ & $\sqrt{ }$ \\
\hline Leave & & & & & & - & - & - & - & - \\
\hline Other Promo & $\sqrt{ }$ & & & $\sqrt{ }$ & & $\sqrt{ }$ & $\sqrt{ }$ & & & \\
\hline Covered Bike Number & $\sqrt{ }$ & & & & & $\sqrt{ }$ & $\sqrt{ }$ & & & \\
\hline Uncovered Bike Number & $\sqrt{ }$ & & & $\sqrt{ }$ & & $\sqrt{ }$ & $\sqrt{ }$ & & & \\
\hline Lockers Number & $\sqrt{ }$ & & & & & $\sqrt{ }$ & $\sqrt{ }$ & & & \\
\hline
\end{tabular}




\begin{tabular}{|c|c|c|c|c|c|c|c|c|c|c|}
\hline \multirow[t]{2}{*}{ Variables } & \multicolumn{5}{|c|}{ NN models on data with all variables } & \multicolumn{5}{|c|}{$\begin{array}{l}\text { Models on data without preference } \\
\text { and other insignificant variables }\end{array}$} \\
\hline & $\mathbf{N}$ & $\mathbf{S}$ & M & C & $\mathbf{E}$ & $\mathbf{N}$ & FE & FR & BK & SP \\
\hline Showers Number & $\sqrt{ }$ & & $\sqrt{ }$ & & & $\sqrt{ }$ & $\sqrt{ }$ & & & \\
\hline Shelters Number & $\checkmark$ & & & & & $\sqrt{ }$ & $\sqrt{ }$ & & & \\
\hline Other Amenities 1 Number & $v$ & & & & $\checkmark$ & $\sqrt{ }$ & $\sqrt{ }$ & $\checkmark$ & $\sqrt{ }$ & $\mathrm{v}$ \\
\hline Carpool Spaces Number & $v$ & & & & & $\sqrt{ }$ & $\sqrt{ }$ & & & \\
\hline Vanpool Spaces Number & $\checkmark$ & & & & & $\sqrt{ }$ & $\sqrt{ }$ & & & \\
\hline soV Parking Charge & $\checkmark$ & & & & & $\sqrt{ }$ & $\checkmark$ & & $\checkmark$ & \\
\hline SOV Parking Charge Number & $v$ & & $\checkmark$ & & & $\sqrt{ }$ & $\sqrt{ }$ & & & \\
\hline Reduced SOVP Number & $v$ & $\checkmark$ & & & & $\sqrt{ }$ & $\sqrt{ }$ & & $\checkmark$ & \\
\hline Transit Subsidy & $v$ & & & & & $\sqrt{ }$ & $\sqrt{ }$ & & & \\
\hline Ferry Subsidy & $\checkmark$ & & & & $\checkmark$ & $\sqrt{ }$ & $\sqrt{ }$ & & & \\
\hline Vanpool Subsidy & $v$ & & & & & $\sqrt{ }$ & $\sqrt{ }$ & & & \\
\hline Carpool Subsidy & $v$ & & & & & $\sqrt{ }$ & $\sqrt{ }$ & & & \\
\hline Walking Subsidy & $\checkmark$ & & & & & $\sqrt{ }$ & $\sqrt{ }$ & & & \\
\hline Bike Subsidy & $\checkmark$ & & & & & $\sqrt{ }$ & $\checkmark$ & & & \\
\hline Employees on flextime & $\checkmark$ & & & & & $\sqrt{ }$ & $\sqrt{ }$ & & & \\
\hline Employees with GRH & $\checkmark$ & & & & $\sqrt{ }$ & $\sqrt{ }$ & $\checkmark$ & & & \\
\hline Employees in-house match & $\checkmark$ & & $\sqrt{ }$ & & & $\sqrt{ }$ & $\checkmark$ & & & \\
\hline Employees public match & $\checkmark$ & & & $\sqrt{ }$ & $\sqrt{ }$ & $\sqrt{ }$ & $\sqrt{ }$ & & & \\
\hline FV work employees & $v$ & & & & & $\sqrt{ }$ & $\sqrt{ }$ & & & \\
\hline Number of Employees & $\checkmark$ & & & $\sqrt{ }$ & & $\sqrt{ }$ & $\sqrt{ }$ & & & \\
\hline Percentages of employees on $3 / 36 \mathrm{cWW}$ & $\checkmark$ & & $\sqrt{ }$ & $v$ & & $\sqrt{ }$ & $\sqrt{ }$ & $\sqrt{ }$ & $\checkmark$ & $\sqrt{ }$ \\
\hline Percentages of employees on $4 / 40 \mathrm{CWW}$ & $\checkmark$ & $\checkmark$ & $\sqrt{ }$ & $v$ & & $\sqrt{ }$ & $\sqrt{ }$ & $\sqrt{ }$ & & $\sqrt{ }$ \\
\hline Percentages of employees on $5 / 40$ & $\checkmark$ & $\checkmark$ & $\sqrt{ }$ & & & $\sqrt{ }$ & $\sqrt{ }$ & & & \\
\hline Percentages of employees on $7 / 40 \mathrm{CWW}$ & $\checkmark$ & & & & & $\sqrt{ }$ & $\checkmark$ & $\checkmark$ & & $\checkmark$ \\
\hline Percentages of employees on $9 / 80 \mathrm{cWW}$ & $\checkmark$ & & & $\checkmark$ & & $\sqrt{ }$ & $\sqrt{ }$ & & & \\
\hline Percentages of employees on other CWW & $\checkmark$ & & & & & $\sqrt{ }$ & $\sqrt{ }$ & $\checkmark$ & $\checkmark$ & $\checkmark$ \\
\hline Alone share & $\checkmark$ & $\checkmark$ & & $\sqrt{ }$ & & $\sqrt{ }$ & & & & \\
\hline Bike share & $v$ & & & $\sqrt{ }$ & & $\sqrt{ }$ & $\sqrt{ }$ & $\checkmark$ & & $\checkmark$ \\
\hline Bus share & $\mathrm{v}$ & $\sqrt{ }$ & $\checkmark$ & $\sqrt{ }$ & $\sqrt{ }$ & $\sqrt{ }$ & $\sqrt{ }$ & & $\checkmark$ & \\
\hline Cars hare & $\checkmark$ & $\sqrt{ }$ & $\checkmark$ & $\sqrt{ }$ & $\sqrt{ }$ & $\sqrt{ }$ & $\sqrt{ }$ & $\sqrt{ }$ & & $\sqrt{ }$ \\
\hline Other share & $\checkmark$ & $\sqrt{ }$ & $\sqrt{ }$ & & & $\sqrt{ }$ & $\sqrt{ }$ & $\sqrt{ }$ & & $\sqrt{ }$ \\
\hline Tele share & $\checkmark$ & & & & & $\sqrt{ }$ & $\sqrt{ }$ & & & \\
\hline Van share & $\checkmark$ & & & & & $\sqrt{ }$ & $\sqrt{ }$ & $\sqrt{ }$ & & $\sqrt{ }$ \\
\hline Walk share & $\checkmark$ & & & $\sqrt{ }$ & & $\sqrt{ }$ & $\checkmark$ & & $\checkmark$ & \\
\hline Days saved telecommuting in two weeks & $\checkmark$ & & & & & 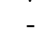 & - & - & - & - \\
\hline Administration job Share & $\checkmark$ & $\checkmark$ & $\checkmark$ & $\sqrt{ }$ & & - & - & - & - & - \\
\hline Craft/Production/Labor Share & $\checkmark$ & & & $\sqrt{ }$ & & - & - & - & - & - \\
\hline Management job Share & $\checkmark$ & & & & & - & - & - & - & - \\
\hline Sales/Marketing job Share & $\mathrm{v}$ & $\checkmark$ & & & & - & - & - & - & - \\
\hline Customer Service job Share & $\checkmark$ & & & & & - & - & - & - & - \\
\hline Other job Share & $\checkmark$ & & & & $\checkmark$ & - & - & - & - & - \\
\hline Professional/Technical job Share & $\checkmark$ & & & & & - & - & - & - & - \\
\hline Q8_Invalid_Share & $\checkmark$ & & $\checkmark$ & & & - & - & - & - & - \\
\hline Prefer provide car for work & $\checkmark$ & & & & & - & - & - & - & - \\
\hline Employee Prefer Transport during lunch & $\checkmark$ & & & & & - & - & - & - & - \\
\hline Employee Prefer GRH & $\checkmark$ & & & & & - & - & - & - & - \\
\hline Employee Prefer flex to meet CVpool bus & $\checkmark$ & & & & $\sqrt{ }$ & - & - & - & - & - \\
\hline Employee Prefer financial incentive & $\mathrm{v}$ & & & $\sqrt{ }$ & & - & - & - & - & - \\
\hline $\begin{array}{l}\text { Employee Prefer reserved discounted } \\
\text { CVpool space }\end{array}$ & $\checkmark$ & $\sqrt{ }$ & $\sqrt{ }$ & & $\sqrt{ }$ & - & - & - & - & - \\
\hline $\begin{array}{l}\text { Employee Prefer Personalized help for } \\
\text { CVpool }\end{array}$ & $\checkmark$ & & & & $\sqrt{ }$ & - & - & - & - & - \\
\hline Employee Prefer covered bicycle parking & $\checkmark$ & $\checkmark$ & $\checkmark$ & & & - & - & - & - & - \\
\hline Employee Prefer lockers \& showers & $\checkmark$ & & & & & - & - & - & - & - \\
\hline Employee Prefer onsite childcare & $\checkmark$ & & & & & - & - & - & - & - \\
\hline Employee Prefer CWW & $\checkmark$ & & & & & - & - & - & - & - \\
\hline Employee Prefer to telecommute & $\checkmark$ & & & $\sqrt{ }$ & & - & - & - & - & - \\
\hline $\begin{array}{l}\text { Employee Prefer improved access to } \\
\text { transit }\end{array}$ & $\checkmark$ & & $\checkmark$ & & $\checkmark$ & - & - & - & - & - \\
\hline sov & $\checkmark$ & $\sqrt{ }$ & $\checkmark$ & $\sqrt{ }$ & & - & - & - & - & - \\
\hline VMT & $\checkmark$ & & & & & - & - & - & - & - \\
\hline VTR & $\checkmark$ & $\sqrt{ }$ & & $\checkmark$ & $\checkmark$ & $\sqrt{ }$ & $\sqrt{ }$ & & $\sqrt{ }$ & \\
\hline
\end{tabular}




\begin{tabular}{|c|c|c|c|c|c|c|c|c|c|c|}
\hline \multirow{2}{*}{ Variables } & \multicolumn{5}{|c|}{ NN models on data with all variables } & \multicolumn{5}{|c|}{$\begin{array}{l}\text { Models on data without preference } \\
\text { and other insignificant variables }\end{array}$} \\
\hline & $\mathbf{N}$ & $\mathbf{S}$ & $M$ & C & $\mathbf{E}$ & $\mathbf{N}$ & FE & FR & BK & SP \\
\hline $\begin{array}{l}\text { Central Business District } \\
\text { Suburban area } \\
\text { Outside suburban area }\end{array}$ & $\begin{array}{l}\sqrt{ } \\
\sqrt{ } \\
\sqrt{ }\end{array}$ & & & & & $\begin{array}{l}\sqrt{ } \\
\sqrt{ } \\
\sqrt{ }\end{array}$ & $\begin{array}{l}\sqrt{ } \\
\sqrt{ } \\
\sqrt{ }\end{array}$ & & & \\
\hline
\end{tabular}

From Figures 50 and 51, it can be seen that the neural network model built with no variable selection on data with all variables was able to get the best 'bin classification accuracy in moderate range of change in VTR' (24.59 percent) and 'best bin classification accuracy on full range of change in VTR' (19.44 percent) with an R-square value of 0.052 . Since this model includes the preference variables, it again shows the importance of the employee's preferences towards incentives in predicting the changes in VTR.

The better models, excluding the previous model, are the forward/stepwise regression (M1) and forced enter regression (M2) models on the condensed data. The forward and stepwise regression models selected the same variables and regression coefficients, resulting in identical results. The detailed accuracies of these models on the each of the bins are shown in Table 36.

Table 34: Detailed accuracies on bins

\begin{tabular}{|c|c|c|c|c|c|c|c|c|c|c|c|c|}
\hline & \multirow[b]{2}{*}{ Range } & \multirow[b]{2}{*}{$\begin{array}{c}\text { \# of } \\
\text { records }\end{array}$} & \multicolumn{8}{|c|}{ Bin ranges over Change in Vehicle Trip Rate (Delta_VTR) } & \multirow{6}{*}{$\begin{array}{l}\text { Weighted } \\
\text { Avg on } \\
\text { a2 to a5 }\end{array}$} & \multirow{5}{*}{ R-square } \\
\hline & & & $>-7$ & \begin{tabular}{|c}
{$[-7$ to -} \\
$4.5)$
\end{tabular} & $\begin{array}{c}{\left[\begin{array}{c}-4.5 \\
-3)\end{array}\right.} \\
\text { to }\end{array}$ & $\begin{array}{c}{[-3 \text { to }-} \\
1.5)\end{array}$ & $\begin{array}{c}{[-1.5 \text { to }} \\
0)\end{array}$ & $\begin{array}{l}\text { [0 to } \\
1.5)\end{array}$ & $\begin{array}{c}{[1.5 \text { to }} \\
3.5)\end{array}$ & $3.5>=$ & & \\
\hline & Bin Number & & a1 & a2 & a3 & A4 & a5 & a6 & a7 & a8 & & \\
\hline & Validation & 144 & 15 & 15 & 15 & 17 & 20 & 20 & 21 & 21 & & \\
\hline & Training & 1862 & 150 & 172 & 282 & 341 & 306 & 214 & 178 & 219 & & \\
\hline & & & & & & & & & & & & \\
\hline M1 & Exact Validation & $15.28 \%$ & $0.00 \%$ & $0.00 \%$ & $0.00 \%$ & $50.00 \%$ & $43.75 \%$ & $15.38 \%$ & $0.00 \%$ & $13.64 \%$ & $23.77 \%$ & 0.079 \\
\hline & Exact Training & $19.60 \%$ & $0.67 \%$ & $0.00 \%$ & $12.77 \%$ & $48.97 \%$ & $40.85 \%$ & $8.88 \%$ & $5.62 \%$ & $3.20 \%$ & $26.63 \%$ & 0.090 \\
\hline & One-off Validation & $35.42 \%$ & $0.00 \%$ & $17.65 \%$ & $0.00 \%$ & $87.50 \%$ & $87.50 \%$ & $42.31 \%$ & $5.56 \%$ & $13.64 \%$ & $49.39 \%$ & \\
\hline & One-Off Training & $51.61 \%$ & $1.33 \%$ & $12.21 \%$ & $58.87 \%$ & $93.84 \%$ & $90.52 \%$ & $50.47 \%$ & $20.79 \%$ & $13.70 \%$ & $23.77 \%$ & 0.079 \\
\hline M2 & Exact Validation & $12.50 \%$ & $5.88 \%$ & $5.88 \%$ & $0.00 \%$ & $31.25 \%$ & $37.50 \%$ & $11.54 \%$ & $0.00 \%$ & $4.55 \%$ & $19.16 \%$ & 0.090 \\
\hline & Exact Training & $18.69 \%$ & $1.33 \%$ & $4.65 \%$ & $13.12 \%$ & $41.35 \%$ & $35.29 \%$ & $14.49 \%$ & $7.87 \%$ & $3.20 \%$ & $24.36 \%$ & 0.121 \\
\hline & One-off Validation & $38.89 \%$ & $11.76 \%$ & $23.53 \%$ & $0.00 \%$ & $81.25 \%$ & $81.25 \%$ & $46.15 \%$ & $5.56 \%$ & $27.27 \%$ & $47.85 \%$ & \\
\hline & One-Off Training & $52.95 \%$ & $6.00 \%$ & $25.00 \%$ & $60.64 \%$ & $91.50 \%$ & $82.68 \%$ & $50.93 \%$ & $28.09 \%$ & $17.81 \%$ & & \\
\hline
\end{tabular}

It can be seen from the table, that for forwards forward/stepwise regressions models the accuracy of bins $a 1, a 2, a 3$ and $a 7$ is zero. This makes the model unsuitable as we would like to have accuracy spread out over all the bins. 
Actual Delta_VTR Vs. Predicted Delta_VTR

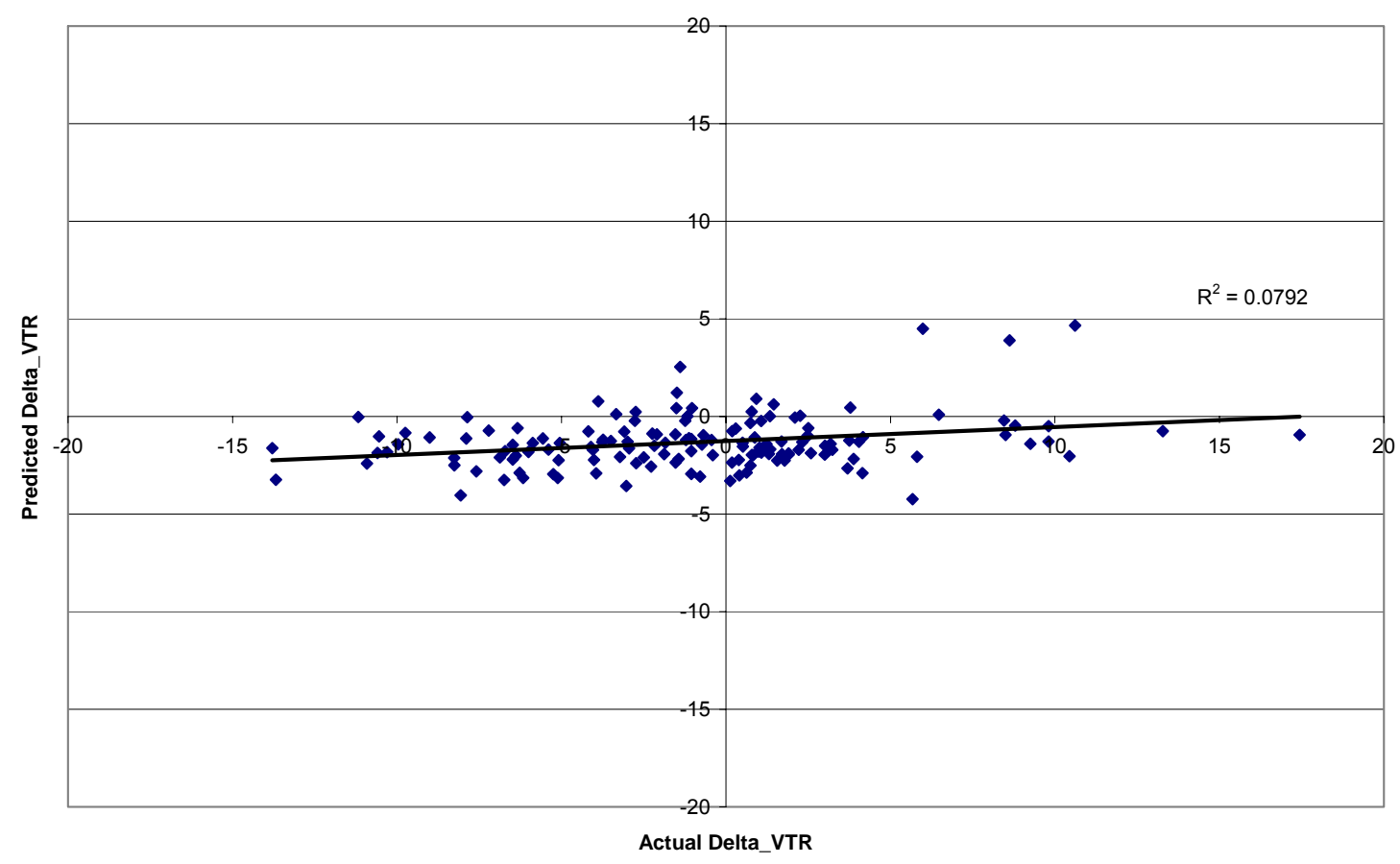

Figure 53 : Scatter plot for forward/stepwise regression model

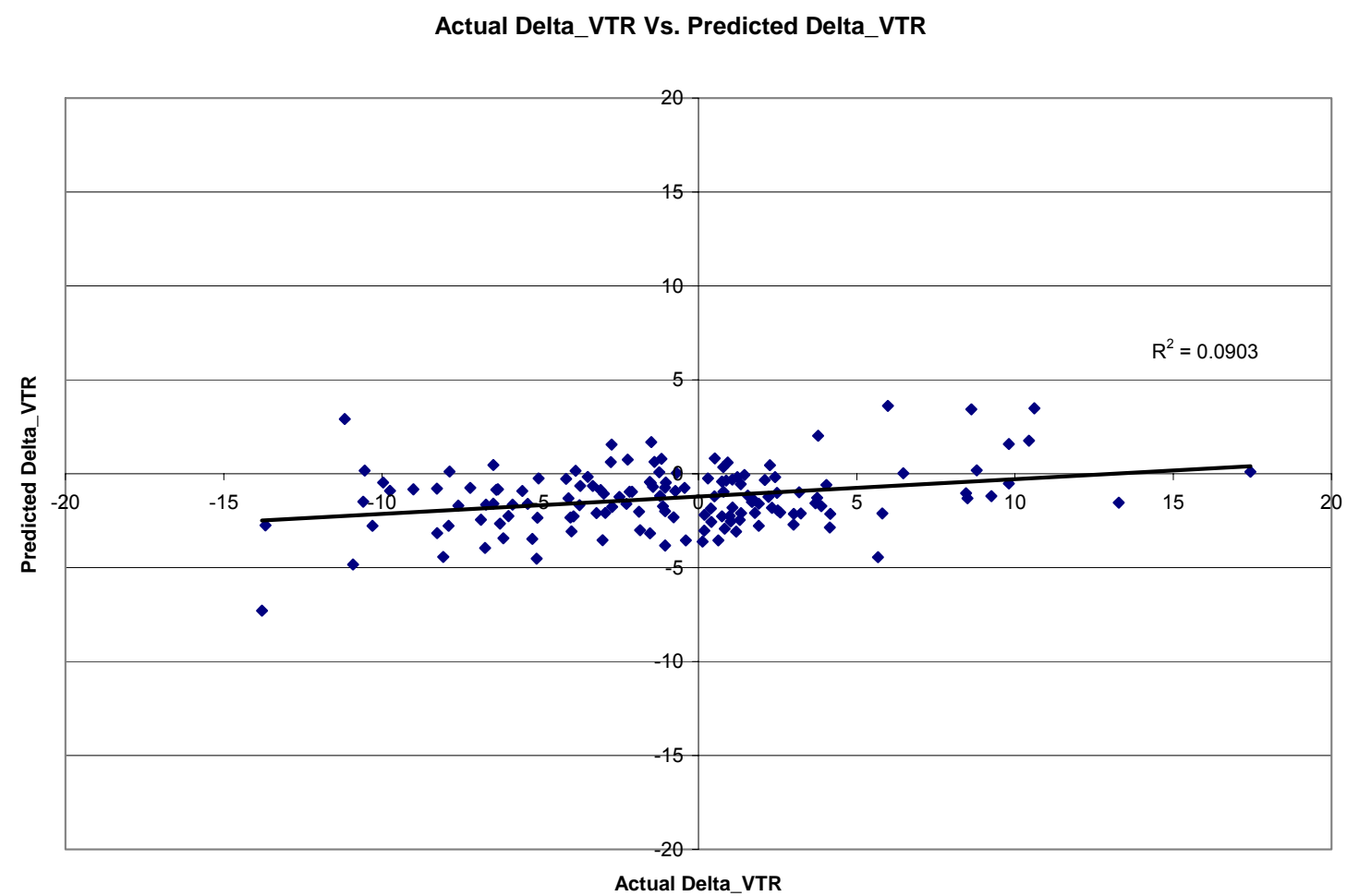

Figure 54: Scatter plot for forced enter regression model 
The regression coefficients for the forced enter regression model (M2) are shown in Table 37.

Table 35: Variables and coefficients for forced enter regression model

\begin{tabular}{|c|c|c|}
\hline Variable & coefficient & t - value \\
\hline Constant & 33.155 & 2.177 \\
\hline Health organization & -0.359 & -0.511 \\
\hline Retail organization & -1.367 & -1.422 \\
\hline Manufacturing organization & 1.026 & 1.908 \\
\hline other organization & 0.459 & 1.479 \\
\hline Shifts & 0.521 & 1.943 \\
\hline Onsite Parking Spaces & -0.067 & -0.473 \\
\hline Offsite Parking Spaces & -0.787 & -1.805 \\
\hline Leased Onsite Parking Price & -0.001 & -0.371 \\
\hline Leased Offsite Parking Price & -0.005 & -1.206 \\
\hline Own Onsite Parking & 0.791 & 2.886 \\
\hline Onsite Parking Charge & -0.001 & -0.168 \\
\hline Own Offsite Parking & -0.412 & -0.844 \\
\hline Offsite Parking Charge & 0.006 & 0.830 \\
\hline Pay Parking Charge & 0.006 & 1.453 \\
\hline on off parking sub & -1.519 & -2.835 \\
\hline Free Parking $1 / 4$ mile & 0.368 & 1.403 \\
\hline ETC Additional Training & 0.110 & 0.444 \\
\hline ETC Onsite & -0.082 & -0.266 \\
\hline Distribute Info & 0.004 & 1.344 \\
\hline Post Materials & 0.001 & 0.437 \\
\hline CTR Orientation & 0.000 & 0.763 \\
\hline CTR Events & -0.122 & -3.177 \\
\hline CTR E-mail & -0.005 & -0.718 \\
\hline Articles & 0.020 & 1.049 \\
\hline Ride match Apps & -0.002 & -1.187 \\
\hline With Paychecks & 0.011 & 0.324 \\
\hline Drawings & 0.050 & 2.062 \\
\hline Other Promo & 0.005 & 1.017 \\
\hline Covered Bike Number & 0.264 & 0.232 \\
\hline Uncovered Bike Number & 2.908 & 0.892 \\
\hline Lockers Number & -0.068 & -0.181 \\
\hline Showers Number & -5.687 & -1.536 \\
\hline Shelters Number & -1.026 & -0.315 \\
\hline Other Amenities 1 Number & -22.516 & -3.710 \\
\hline Carpool Spaces Number & -1.373 & -1.608 \\
\hline Vanpool Spaces Number & 2.920 & 0.916 \\
\hline SOV Parking Charge & -0.009 & -1.938 \\
\hline SOV Parking Charge Number & 0.443 & 1.490 \\
\hline Reduced SOVP Number & 2.798 & 1.652 \\
\hline Transit Subsidy & 0.000 & -0.003 \\
\hline Ferry Subsidy & 0.014 & 1.410 \\
\hline Vanpool Subsidy & -0.022 & -2.242 \\
\hline Carpool Subsidy & 0.001 & 0.066 \\
\hline Walking Subsidy & 0.014 & 0.468 \\
\hline Bike Subsidy & 0.002 & 0.068 \\
\hline employees on flextime & 0.350 & 0.686 \\
\hline employees with GRH & 0.324 & 0.917 \\
\hline employees in-house match & -0.191 & -1.328 \\
\hline employees public match & -0.113 & -1.345 \\
\hline FV work employees & -0.043 & -0.340 \\
\hline Number of Employee & 0.000 & -0.517 \\
\hline Percentages of employees on $3 / 36 \mathrm{CWW}$ & 7.452 & 0.799 \\
\hline Percentages of employees on $4 / 40 \mathrm{CWW}$ & -2.340 & -0.731 \\
\hline Percentages of employees on $5 / 40$ & -1.600 & -1.029 \\
\hline Percentages of employees on $7 / 40 \mathrm{CWW}$ & -3.723 & -0.651 \\
\hline
\end{tabular}




\begin{tabular}{|l|r|r|}
\hline \multicolumn{1}{|c|}{ Variable } & coefficient & t - value \\
\hline Percentages of employees on 9/80 CWW & -4.338 & -1.842 \\
Percentages of employees on other CWW & -15.095 & -3.142 \\
Bike share & -6.940 & -0.440 \\
Bus share & -30.244 & -2.070 \\
Cars hare & -7.566 & -0.909 \\
Other share & -14.234 & -0.819 \\
Tele share & -27.542 & -1.443 \\
Van share & -6.184 & -0.376 \\
Walk share & -35.160 & -2.316 \\
VTR & -0.334 & -2.252 \\
Central Business District & -3.219 & -1.154 \\
Suburban area & -2.928 & -1.059 \\
Outside suburban area & -2.520 & -0.914 \\
\hline
\end{tabular}

When the scatter plot in figure 54 for this model is compared with the scatter plot in figure 43 for equivalent forced enter regression model built on the full sampled ungrouped incentive data, we can see that the model on oversampled data is biased towards predicting negative changes in VTR. 
Equivalent grouped incentives models were also built on the over-sampled data. Figures 55, 56, and 57 show the accuracies and the R-square values of the neural network and the regression models built using no variable selection.

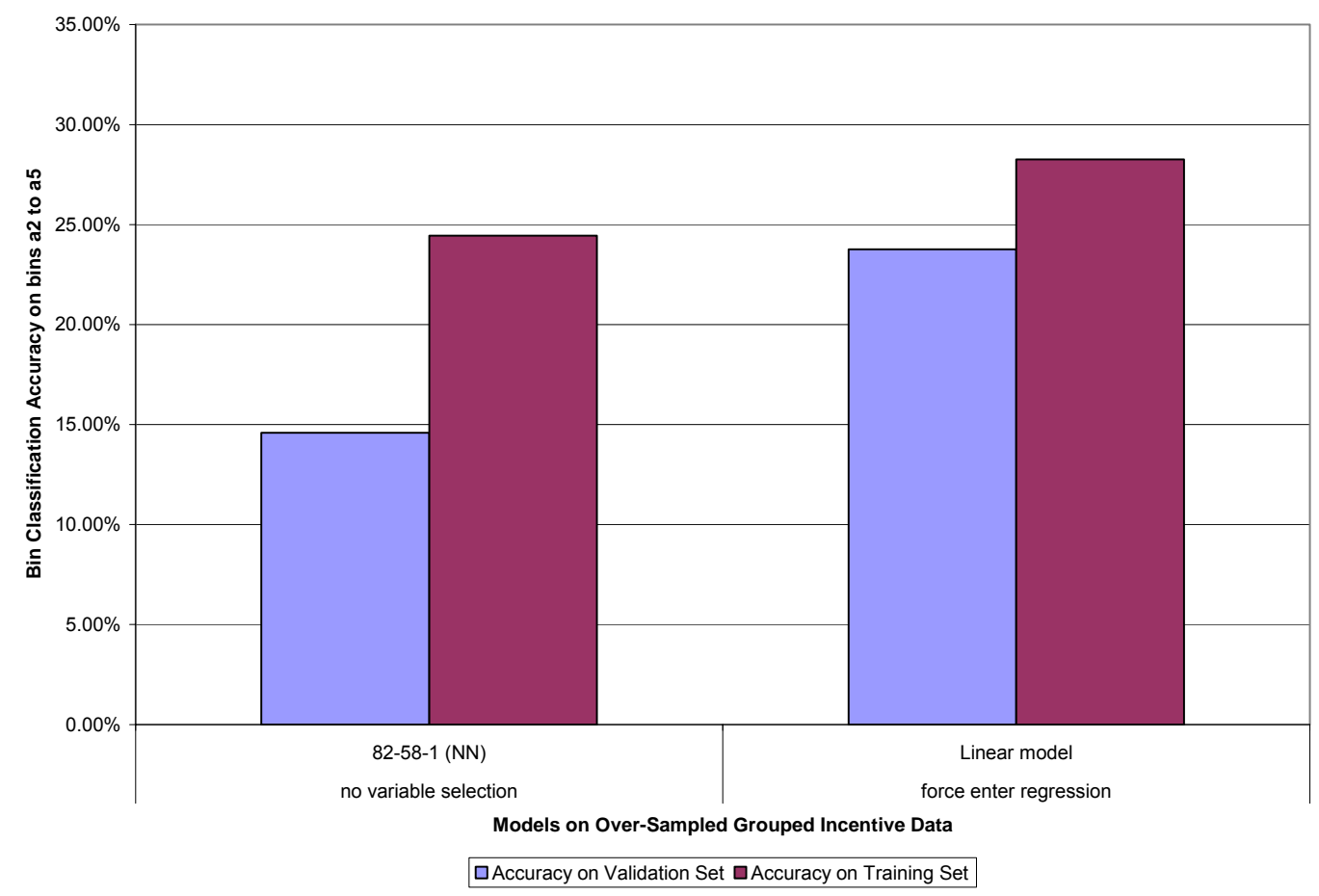

Figure 55: Bin Classification Accuracy on Moderate Range of change in VTR (a2 to a5) for validation \& training set (Different models on Over-Sampled Grouped Incentive data)

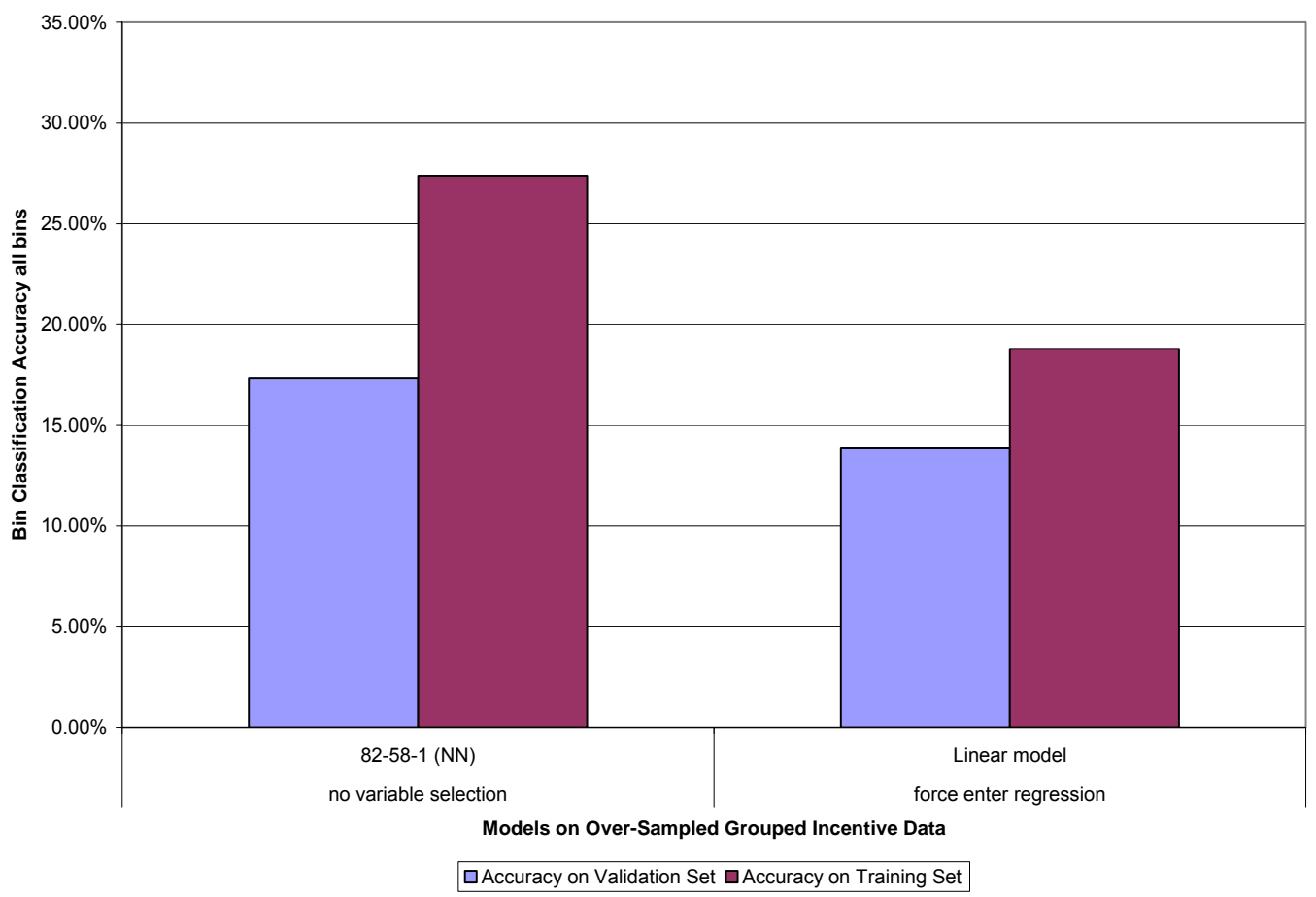

Figure 56: Bin Classification Accuracy on Full Range of change in VTR (all bins) for validation \& training set (Different models on Over-Sampled Grouped Incentive data) 


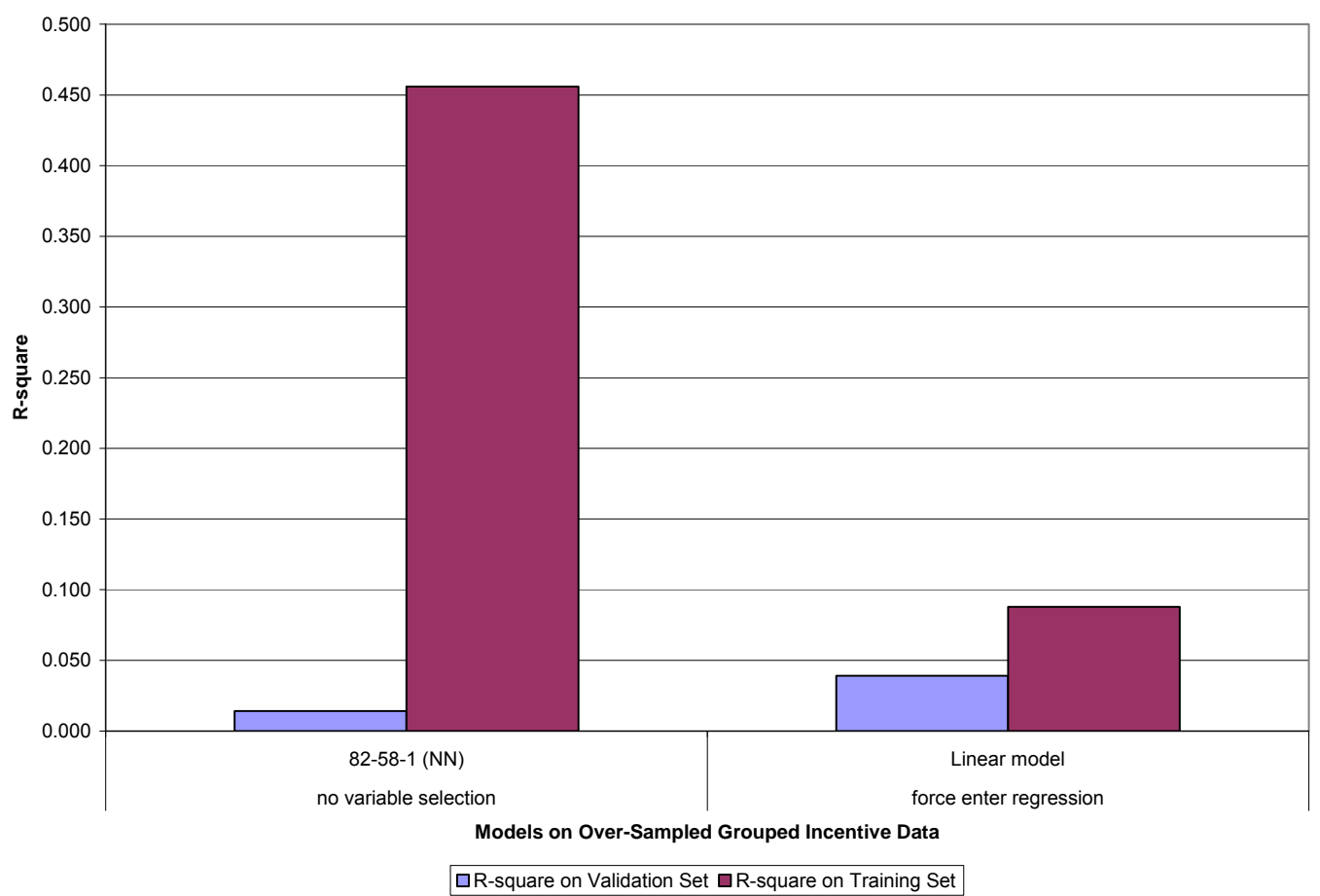

Figure 57: R-square for validation \& training set (Different models on Over-Sampled Grouped Incentive data)

The charts in Figures 55, 56, and 57 display that none of the models beat the others in all of the performance measures. The Neural network (M1) and linear regression (M2) built on this over-sampled data seem to do better than the corresponding models built on non-over-sampled data. The accuracies for both of the models are shown in Table 38.

Table 36: Detailed accuracies on bins

\begin{tabular}{|c|c|c|c|c|c|c|c|c|c|c|c|c|}
\hline & \multirow[b]{2}{*}{ Range } & \multirow{2}{*}{$\begin{array}{c}\text { \# of } \\
\text { records }\end{array}$} & \multicolumn{8}{|c|}{ Bin ranges over Change in Vehicle Trip Rate (Delta_VTR) } & \multirow{4}{*}{$\begin{array}{l}\text { Weighted } \\
\text { Avg on } \\
\text { a2 to a5 }\end{array}$} & \multirow[b]{3}{*}{ R-square } \\
\hline & & & $>-7$ & $\begin{array}{c}{[-7 \text { to }-} \\
4.5)\end{array}$ & $\mid \begin{array}{c}{[-4.5 \text { to }} \\
-3)\end{array}$ & $\begin{array}{c}{[-3 \text { to }-} \\
1.5)\end{array}$ & $\begin{array}{c}{[-1.5 \text { to }} \\
0)\end{array}$ & $\begin{array}{l}\text { [0 to } \\
1.5)\end{array}$ & $\begin{array}{c}{[1.5 \text { to }} \\
3.5)\end{array}$ & $3.5>=$ & & \\
\hline & $\begin{array}{c}\text { Bin Number } \\
\text { Validation } \\
\text { Training }\end{array}$ & $\begin{array}{c}144 \\
1862 \\
\end{array}$ & $\begin{array}{c}\mathbf{A 1} \\
15 \\
150 \\
\end{array}$ & $\begin{array}{c}\mathbf{a 2} \\
15 \\
172 \\
\end{array}$ & $\begin{array}{c}\text { a3 } \\
15 \\
282 \\
\end{array}$ & $\begin{array}{c}\mathbf{a 4} \\
17 \\
341 \\
\end{array}$ & $\begin{array}{c}\mathbf{a 5} \\
20 \\
306 \\
\end{array}$ & $\begin{array}{c}\mathbf{a 6} \\
20 \\
214 \\
\end{array}$ & $\begin{array}{c}\mathbf{A 7} \\
21 \\
178 \\
\end{array}$ & $\begin{array}{c}\mathbf{a 8} \\
21 \\
219 \\
\end{array}$ & & \\
\hline & & & & & & & & & & & & \\
\hline M1 & Exact Validation & $17.36 \%$ & $0.00 \%$ & $0.00 \%$ & $8.33 \%$ & $18.75 \%$ & $31.25 \%$ & $19.23 \%$ & $27.78 \%$ & $27.27 \%$ & $14.58 \%$ & 0.014 \\
\hline & Exact Training & $27.39 \%$ & $15.33 \%$ & $13.29 \%$ & $19.78 \%$ & $26.05 \%$ & $33.23 \%$ & $23.26 \%$ & $27.78 \%$ & $53.88 \%$ & $24.45 \%$ & 0.456 \\
\hline & One-off Validation & $37.50 \%$ & $5.88 \%$ & $11.76 \%$ & $33.33 \%$ & $50.00 \%$ & $50.00 \%$ & $38.46 \%$ & $50.00 \%$ & $54.55 \%$ & $35.79 \%$ & \\
\hline & One-Off Training & $63.86 \%$ & $38.67 \%$ & $40.46 \%$ & $58.63 \%$ & $64.67 \%$ & $76.36 \%$ & $70.23 \%$ & $68.89 \%$ & $76.71 \%$ & $14.58 \%$ & 0.010 \\
\hline M2 & Exact Validation & $13.89 \%$ & $0.00 \%$ & $0.00 \%$ & $0.00 \%$ & $50.00 \%$ & $43.75 \%$ & $11.54 \%$ & $0.00 \%$ & $9.09 \%$ & $23.77 \%$ & 0.039 \\
\hline & Exact Training & $18.80 \%$ & $0.00 \%$ & $0.00 \%$ & $8.99 \%$ & $49.70 \%$ & $38.02 \%$ & $9.77 \%$ & $7.78 \%$ & $2.28 \%$ & $28.26 \%$ & 0.088 \\
\hline & One-off Validation & $34.72 \%$ & $0.00 \%$ & $0.00 \%$ & $50.00 \%$ & $87.50 \%$ & $87.50 \%$ & $46.15 \%$ & $5.56 \%$ & $13.64 \%$ & $55.12 \%$ & \\
\hline & One-Off Training & $50.43 \%$ & $2.00 \%$ & $18.50 \%$ & $54.68 \%$ & $95.21 \%$ & $83.39 \%$ & $50.23 \%$ & $21.67 \%$ & $11.87 \%$ & & \\
\hline
\end{tabular}

The scatter plots on the validation set for these models built on oversampled data are shown in Figures 58 and 59. 
Actual Delta_VTR Vs. Predicted Delta_VTR

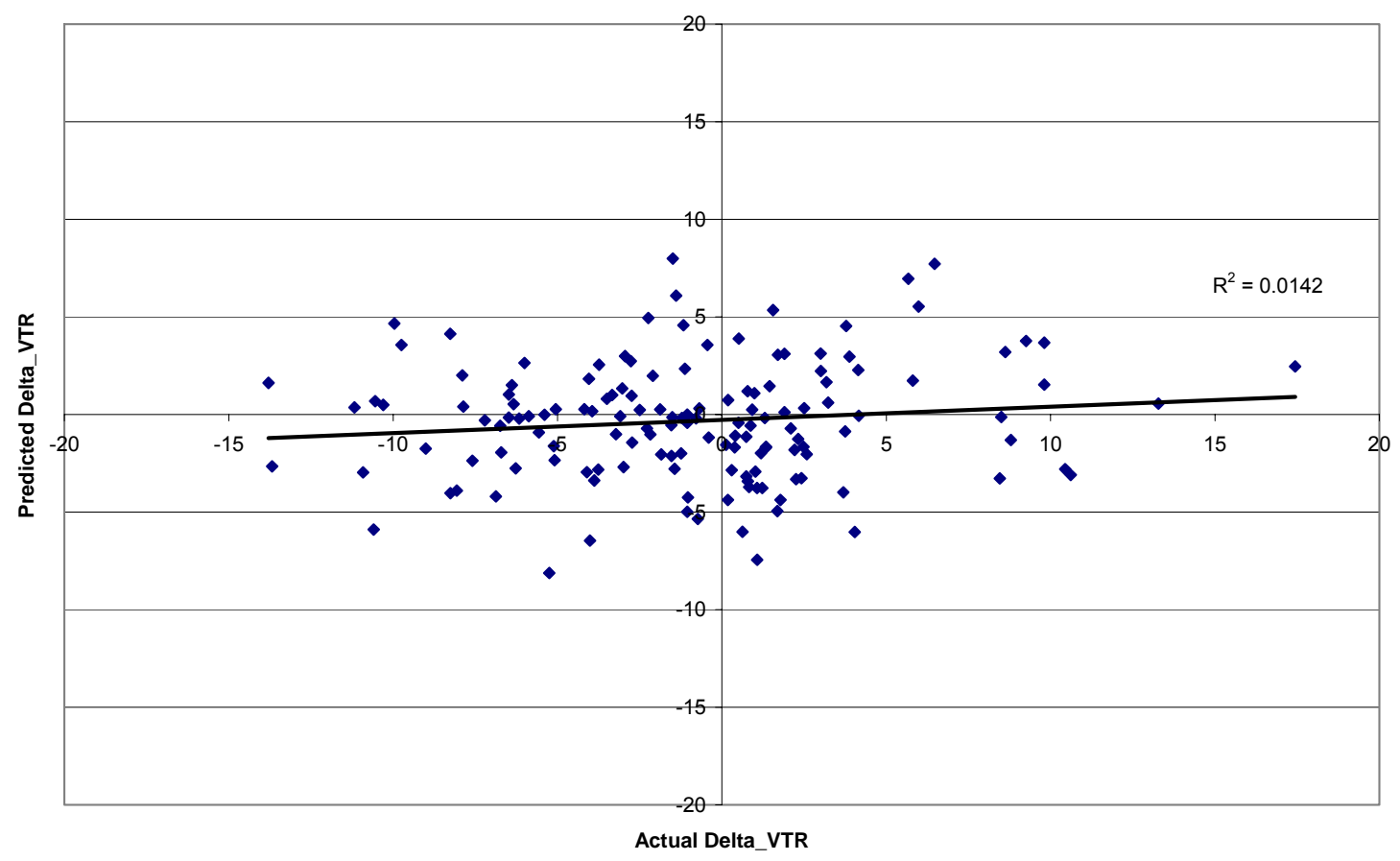

Figure 58: Scatter plot for neural network model

Actual Delta VTR Vs. Predicted Delta VTR

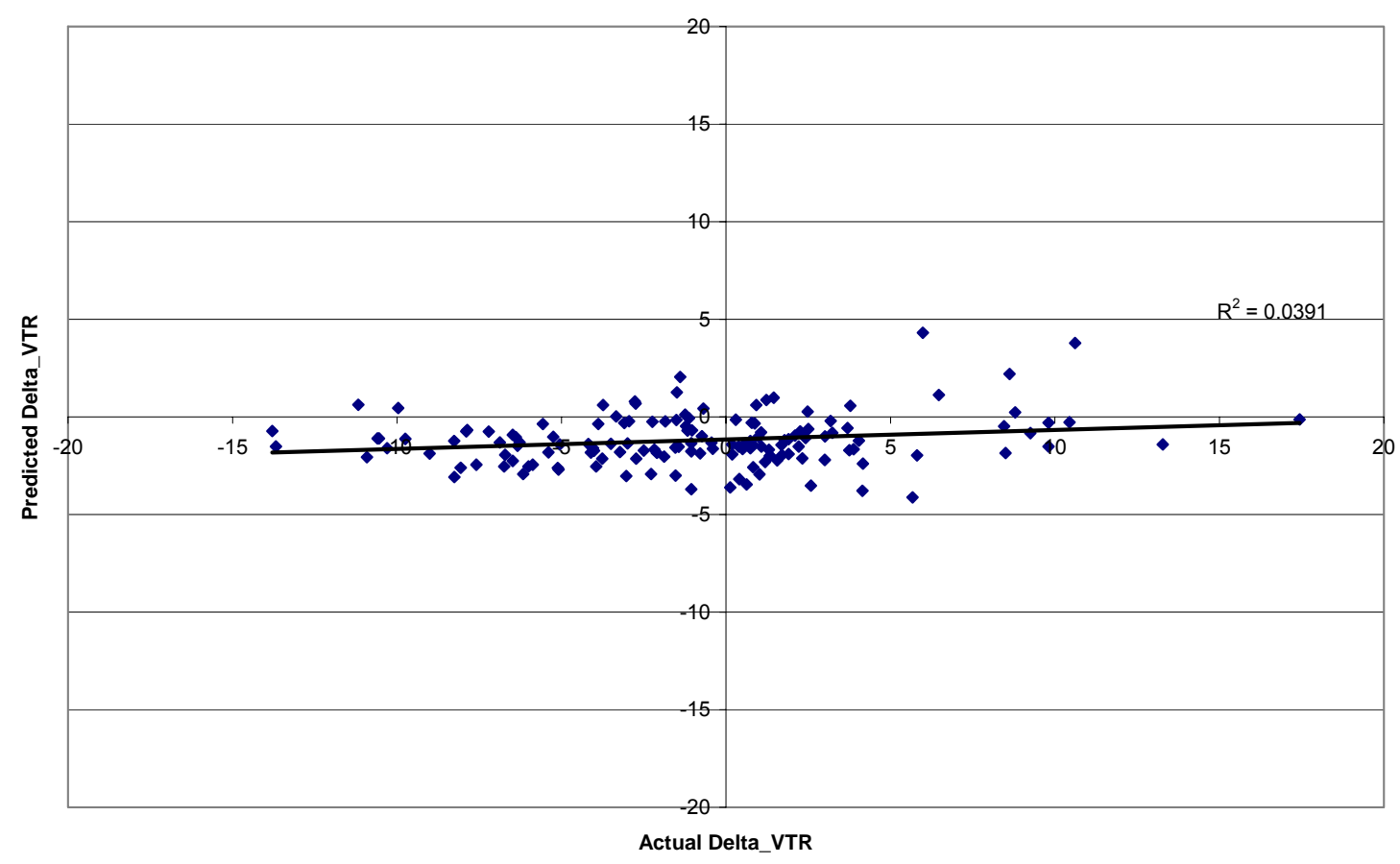

Figure 59: Scatter plot for linear regression model 
From the detailed accuracy table 38 and scatter plot in figure 58, it can be seen that the linear forced enter regression model is biased towards predicting negative changes in VTR which is not the expected behavior of a good model.

The regression coefficients for the forced enter regression model (M2) are shown in Table 39.

Table 37: Variables and coefficients for forced enter regression model

\begin{tabular}{|c|c|c|}
\hline Variables & coefficients & t - value \\
\hline (Constant) & 22.871 & 1.548 \\
\hline Health organization & -0.234 & -0.344 \\
\hline Retail organization & -1.589 & -1.660 \\
\hline Manufacturing organization & 0.411 & 0.817 \\
\hline other organization & 0.023 & 0.083 \\
\hline Shifts & 0.695 & 2.724 \\
\hline covered/uncovered bike parking, lockers a\& showers, shelters, carpool \& vanpool spaces, other amenities & -0.650 & -1.741 \\
\hline Guaranteed ride home program & 0.617 & 2.179 \\
\hline CTR Events, CTR E-mail, Articles, With Paychecks, Other Promo, etc & -0.052 & -0.725 \\
\hline Ridematch Apps, employees in-house match, employees public match & -0.299 & -1.237 \\
\hline Ferry, Carpool, Walking, Bike Subsidy & 0.074 & 0.245 \\
\hline SOV Parking Charge, SOV Parking Charge Number, Reduced SOVP Number & -0.319 & -1.325 \\
\hline Compressed work week $3 / 36,4 / 40,9 / 80,7 / 40$, other & -0.278 & -0.482 \\
\hline onsite incentives & 0.503 & 1.552 \\
\hline Drawing, leaves, etc & 0.619 & 2.125 \\
\hline Transit, vanpool subsidy & -0.038 & -0.141 \\
\hline Number of employees & 0.000 & 0.159 \\
\hline Percentages of employees on $4 / 40 \mathrm{CWW}$ & 1.186 & 0.382 \\
\hline Percentages of employees on $5 / 40$ & 0.225 & 0.157 \\
\hline Percentages of employees on $7 / 40 \mathrm{CWW}$ & 0.057 & 0.010 \\
\hline Percentages of employees on $9 / 80 \mathrm{CWW}$ & -1.278 & -0.566 \\
\hline Percentages of employees on other CWW & -10.221 & -2.134 \\
\hline Bike share & -0.549 & -0.036 \\
\hline Bus share & -22.824 & -1.605 \\
\hline Cars hare & -3.658 & -0.449 \\
\hline Other share & -4.527 & -0.265 \\
\hline Tele share & -30.459 & -1.622 \\
\hline Van share & 0.494 & 0.031 \\
\hline Walk share & -25.494 & -1.724 \\
\hline VTR & -0.253 & -1.750 \\
\hline Central Business District & -2.317 & -0.830 \\
\hline Suburban area & -1.693 & -0.612 \\
\hline Outside suburban area & -1.355 & -0.492 \\
\hline
\end{tabular}




\section{Recommended Model}

The neural network models built in phase I and II on data containing the employee's preferences obtained much better accuracies than the ones that were built without them. This shows the importance of the employee's preferences towards incentives in predicting the changes in VTR. But as stated earlier, the employers may not have access to this employee information. Therefore, these models can only be used for study.

None of the models built without the employee's incentive preferences were exceptionally better from one another. The two models that stood out were the forced enter regression model built on full sample data and the neural network models built on the over-sampled grouped incentive data.

The rationale for considering the forced enter regression model built on full sample data as one of the candidate model was (Table 29-M1)

1. It has 'bin classification accuracy on moderate range of change in VTR' of $19.40 \%$

2. It has a 'R-square' value of 0.047

3. It has 'bin classification accuracy on full range of change in VTR' of $15.97 \%$ which is better than the random choice accuracy of $12.5 \%$

4. It includes all the individual incentives in predicting change in VTR

The rationale for considering the neural network models built on the oversampled grouped incentive data as one of the candidate model was (Table 35-M1)

1. It has the best 'bin classification accuracy on full range of change in VTR' of $17.36 \%$ which is much better than the random choice accuracy of $12.5 \%$

2. It has 'bin classification accuracy on moderate range of change in VTR" of $14.58 \%$

3. It has a 'R-square' value of 0.014

4. It is built on simple grouped incentives variable set.

It was very difficult to select one of these models as the best one. Accordingly, a cross-tab for positive/negative actual changes in VTR against positive/negative predicted changes in VTR on validation and training/testing set was done as shown in tables 40 and 41 .

Table 38 A-D: Validation Set

A

Forced enter regression model on full sample incentive data

Actual Delta VTR / Predicted Delta VTR

Negative

Positive
Negative

56 (TN)

45 (FN)
B

\begin{tabular}{|c|c|c|}
\hline \multicolumn{3}{|c|}{ NN model on over-sampled grouped } \\
incentive data \\
\hline $\begin{array}{c}\text { Actual Delta_VTR / } \\
\text { Predicted Delta_VTR }\end{array}$ & Negative & Positive \\
\hline Negative & 44 & 34 \\
\hline Positive & 38 & 28 \\
\hline
\end{tabular}




\begin{tabular}{|l|c|}
\hline C \\
\hline $\begin{array}{c}\text { Forced enter regression model on } \\
\text { full sample incentive data }\end{array}$ \\
\hline Precision & 0.718 \\
\hline Recall & 0.554 \\
\hline F-value & 0.626 \\
\hline
\end{tabular}

\begin{tabular}{|c|c|}
\hline \multicolumn{2}{|c|}{$\begin{array}{l}\text { NN model on over-sampled grouped } \\
\text { incentive data }\end{array}$} \\
\hline Precision & 0.564 \\
\hline Recall & 0.537 \\
\hline F-value & 0.550 \\
\hline
\end{tabular}

Table 39 A-D: Training/testing Set

A

\begin{tabular}{|c|c|c|}
\hline \multicolumn{3}{|c|}{$\begin{array}{c}\text { Forced enter regression model on full } \\
\text { sample incentive data }\end{array}$} \\
\hline $\begin{array}{c}\text { Actual Delta_VTR / } \\
\text { Predicted Delta_VTR }\end{array}$ & Negative & Positive \\
\hline Negative & 603 & 135 \\
\hline Positive & 355 & 207 \\
\hline
\end{tabular}

C

\begin{tabular}{|l|c|}
\hline \multicolumn{2}{|c|}{$\begin{array}{c}\text { Forced enter regression model on full } \\
\text { sample incentive data }\end{array}$} \\
\hline Precision & $\mathbf{0 . 8 1 7}$ \\
\hline Recall & 0.629 \\
\hline F-value & 0.711 \\
\hline
\end{tabular}

B

\begin{tabular}{|c|c|c|}
\hline \multicolumn{3}{|c|}{ NN model on over-sampled grouped } \\
incentive data \\
\hline $\begin{array}{c}\text { Actual Delta_VTR / } \\
\text { Predicted Delta_VTR }\end{array}$ & Negative & Positive \\
\hline Negative & 932 & 316 \\
\hline Positive & 186 & 428 \\
\hline
\end{tabular}

D

\begin{tabular}{|c|c|}
\hline \multicolumn{2}{|c|}{$\begin{array}{c}\text { NN model on over-sampled grouped } \\
\text { incentive data }\end{array}$} \\
\hline Precision & 0.747 \\
\hline Recall & 0.834 \\
\hline F-value & 0.788 \\
\hline
\end{tabular}

Where,

TN - True Negatives: These are the number of records for which the model predicted a negative change in VTR when the actual change in VTR was also negative (we are more interested in these)

TP - True Positives: These are the number of records for which the model predicted a positive change in VTR when the actual change in VTR was also positive

FP - False Positives: These are the number of records for which the model falsely predicted a positive change in VTR when the actual change in VTR was negative

FN - False Negatives: These are the number of records for which the model falsely predicted a negative change in VTR when the actual change in VTR was also positive

Since we are more interested in the accuracy of the model predicting negative changes in VTR (i.e. we modeling for predicting negative change in VTR), precision gives us the measure of correctness of the model in predicting negative changes in VTR.

Precision $=\frac{\text { True negative }(\mathrm{TN})}{\text { True negative }(\mathrm{TN})+\text { False negative }(\mathrm{FN})}$

Recall gives us the measure of completeness of the model in predicting negative changes in VTR 
Recall $=\frac{\text { True negative }(\mathrm{TN})}{\text { True negative }(\mathrm{TN})+\text { False positive }(\mathrm{FP})}$

$\mathrm{F}-$ value $=\frac{\left(1+\beta^{2}\right) \times \text { Recall } \times \text { Precision }}{\beta^{2} \times \text { Recall }+ \text { Precision }}$

Where $\beta=1$ ( $\beta$ controls relative importance between recall and precision)

Because the desire is to have better accuracies in predicting negative changes in VTR, it would be good to improve the recall without sacrificing the precision. Both of these measures are captured by the F-value and so the goodness of the model can be measured by F-value. It is apparent that the forced enter regression model built on full sample ungrouped incentives data was better than the neural net model built on the over-sampled grouped incentives data. This is because the forced enter regression model has obtained a better $\mathrm{f}$-value on validation set whereas the neural network model got better $f$-value on training set but much less $f$-value on validation set which might be the due to over-fitting of the model on training set and losing its generalization power on validation set. Therefore, the forced enter regression model built on full sample data is the recommended model. 


\section{COMBI NED DATA MODELS}

The grouped incentive data from all the three datasets was combined to build a generalized model. Some of the variables were collapsed into one variable as shown in Table 42 , so that all of the datasets had consistent variables.

Table 40: Variable mapping for combining all three datasets

\begin{tabular}{|c|c|c|c|c|}
\hline Description & Combined Data & Tucson Grouped & LA Grouped & Wash Grouped \\
\hline Alone Share & AloneShare & AloneShare & CAR1 & AloneShr \\
\hline Transit Share & TransitShare & BusShare & TRANSIT & BusShare \\
\hline Carpool +Vanpool Share & CVpoolShare & CVpoolShare & $\begin{array}{l}\text { VAN_CUTR + CAR2 + } \\
\text { CAR3 + CAR4 + CAR5 } \\
+ \text { CAR6 + BUS } \\
\end{array}$ & CpoolShr + VpoolShr \\
\hline Walk Share & WalkShare & WalkShare & WALK & WalkShr \\
\hline Bicycle Share & BcycleShare & McycleShare & BIKE & BikeShr \\
\hline Motorcycle Share & McycleShare & BcycleShare & Motorcycle & OtherShr \\
\hline Telecommute Share & TeleShare & 0 (not present) & TELECOMMUTE & TeleShr \\
\hline 3/36 compressed work week share & CWW336 & CWW336 & CWW336 & CWW336 \\
\hline 4/40 compressed work week share & CWW440 & CWW440 & CWW440 & CWW440 \\
\hline $8 / 80$ compressed work week share & CWW980 & CWW980 & CWW980 & CWW980 \\
\hline facilities \& amenities & FACILITY_AMENITIES & FACILITY_AMENITIES & FACILITY_AMENITIES & FACILITY_AMENITIES \\
\hline Guaranteed ride home programs & GRH & GRH & GRH & GRH \\
\hline flexible timing & FLEX & FLEX & FLEX & FLEX \\
\hline Marketing programs & MRKT & MRKT & MRKT & MRKT \\
\hline Ride share matching programs & RS_MATCH & RS_MATCH & RS_MATCH & RS_MATCH \\
\hline financial incentives & FINANCIAL & FINANCIAL & FINANCIAL & FINANCIAL \\
\hline Parking management & PARKMGT & PARKMGT & PARKMGT & PARKMGT \\
\hline Telecommute program & TELE & TELE & TELE & TELE \\
\hline Compressed work week program & CWW & CWW & CWW & CWW \\
\hline onsite incentives & ONSITE & ONSITE & ONSITE & ONSITE \\
\hline Non financial incentives & DIRECT_NONFINAN & DIRECT_NONFINAN & DIRECT_NONFINAN & DIRECT_NONFINAN \\
\hline commuter tax benefit incentives & COMMTAX & COMMTAX & COMMTAX & COMMTAX \\
\hline Vehicle trip rate & VTR & VTR & VTR & VTR \\
\hline
\end{tabular}




\section{Phase I: Combined Data}

The training data from all the three cities was combined into a single training set while the validation sets for each city were left undisturbed. The number of examples in each bin for three validation and training/testing sets are shown in Table 43.

Table 41: Combined Data - No. of Records in bins

\begin{tabular}{|l|c|c|c|c|c|c|c|c|c|}
\hline Bin Number & Total & a1 & a2 & a3 & a4 & a5 & a6 & a7 & a8 \\
\hline Validation (LA) & $\mathbf{1 8 3 8}$ & 292 & 220 & 261 & 290 & 173 & 157 & 186 & 259 \\
Validation (Tucson) & $\mathbf{1 1 2}$ & 5 & 4 & 9 & 17 & 17 & 21 & 19 & 20 \\
Validation (wash) & $\mathbf{1 4 4}$ & 17 & 17 & 13 & 15 & 16 & 26 & 18 & 22 \\
Train +Test & $\mathbf{1 9 1 7 3}$ & 2729 & 2638 & 2732 & 3287 & 1713 & 1803 & 2009 & 2262 \\
\hline
\end{tabular}

A simple neural network with no variable selection and forced enter linear regression model were built on the data just containing 23 variables shown in Table 42. The models were trained on the combined training set data from three cities and then the model was independently evaluated on the separate validation sets from these cities. These validation sets were the same validation sets used in evaluating the models built independently on each city data. Figures 60,61 and 62 show the comparison of the bin classification accuracies and R-square values of the models built with the combined data and the recommended models built separately on the three datasets.

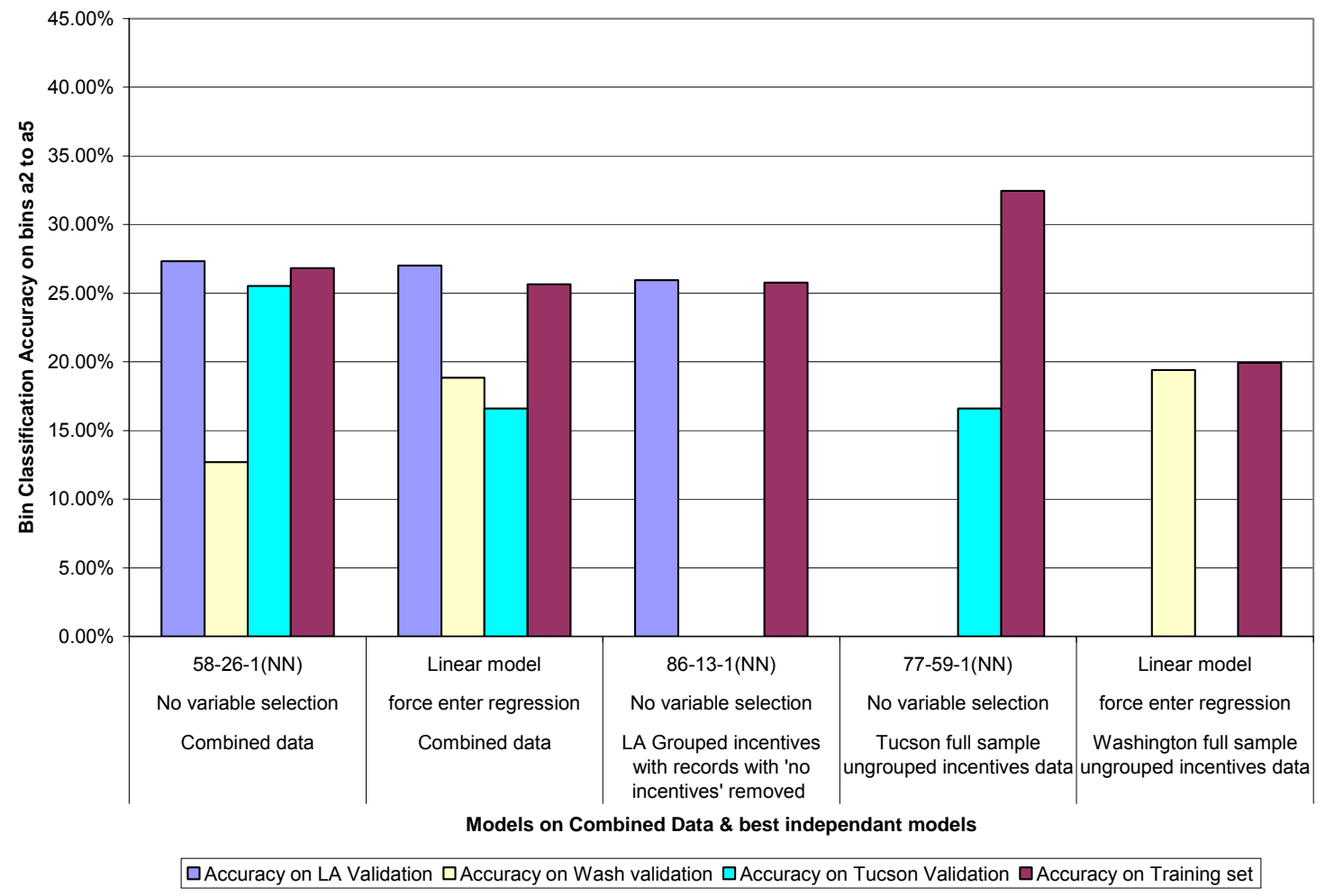

Figure 60: Bin Classification Accuracy on Moderate Range of change in VTR (a2 to a5) for three data sets (Models on combined data $\&$ best independent models) 


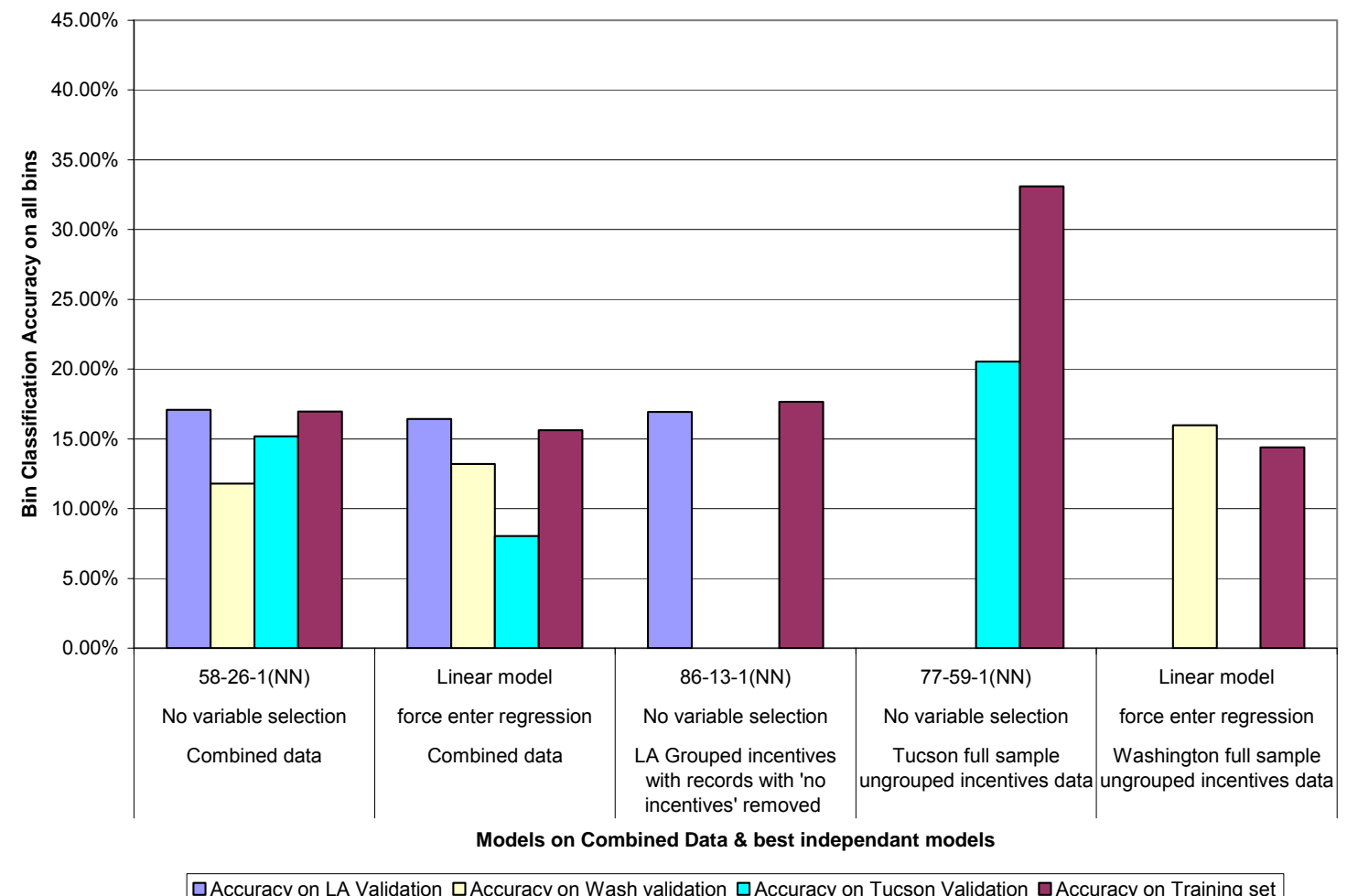

Figure 61: Bin Classification Accuracy on Full Range of change in VTR (all bins) for three validation \& training sets (Models on combined data \& best independent models)

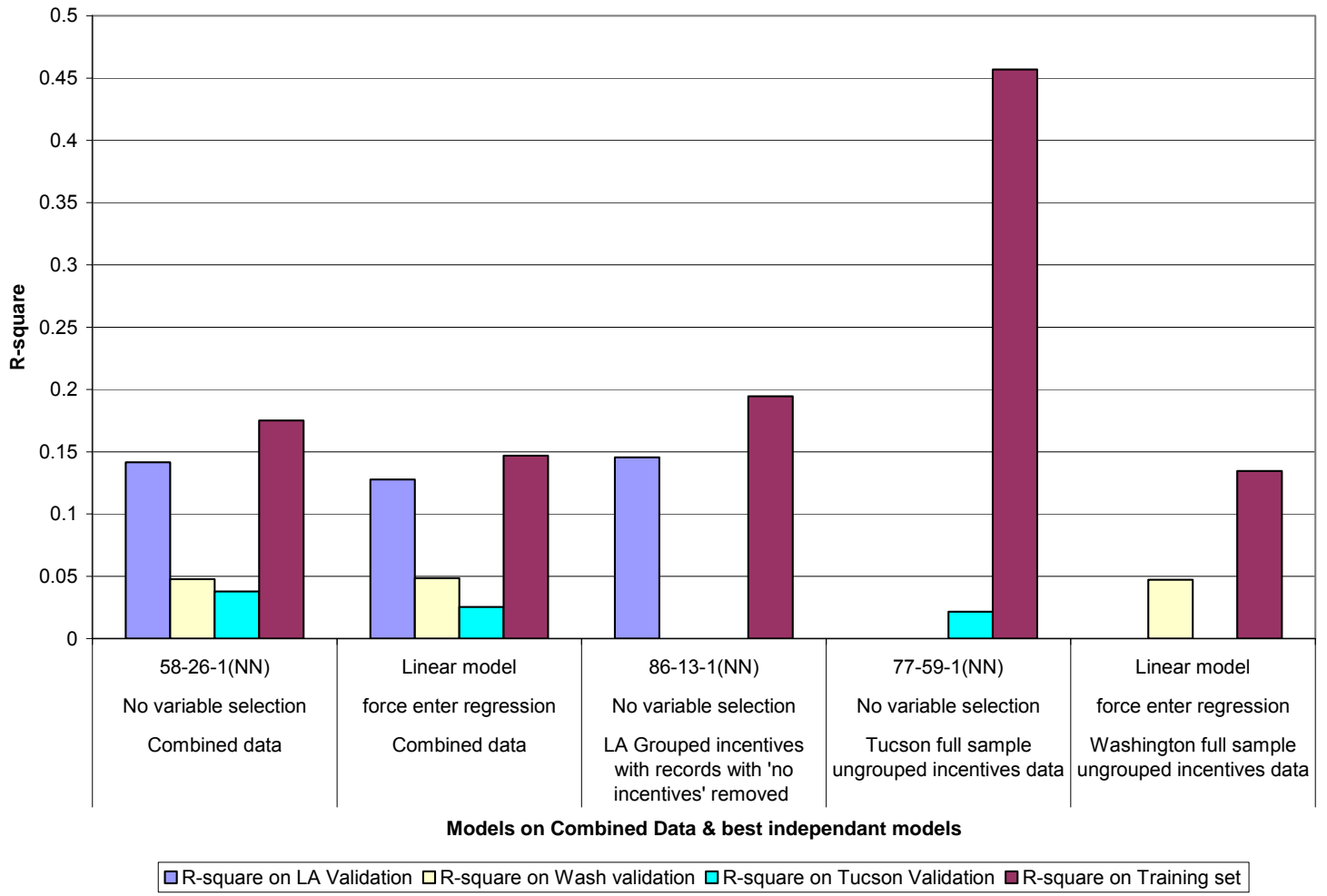

Figure 62: R-square for three validation \& training set (Models on combined data \& best independent models) 
The results in Figure 60, show that the neural net model built on the combined data with no variable selection was able to get better 'bin classification accuracy on moderate range of change in VTR' on the Los Angeles validation set (27.33 percent) as compared to the recommended independent Los Angeles model (25.95 percent) built on grouped incentive dataset and also better 'bin classification accuracy on moderate range of change in VTR' on the Tucson validation set (25.53 percent) as compared to the recommended independent Tucson model (16.6 percent) built on full sample ungrouped incentive dataset. Neither of the models built with combined data were able to improve the accuracy of the Washington validation data.

From figure 61, it was found that this neural net model built on the combined data with no variable selection was able to obtain better 'bin classification accuracy on full range of change in VTR' on the Los Angeles validation set (17.08 percent) as compared to the recommended independent Los Angeles model (16.92 percent). There was considerable reduction in 'bin classification accuracy on full range of change in VTR' on the Tucson and Washington validation sets when compared to result got by independent recommended models.

Figure 62 shows that some improvement in R-square value was obtained by combined data neural net model on the Tucson validation set. The scatter plots for the neural net model built on the combined data of the three validation sets are shown in Figures 63, 64, and 65.

Actual Delta_VTR Vs. Predicted Delta_VTR

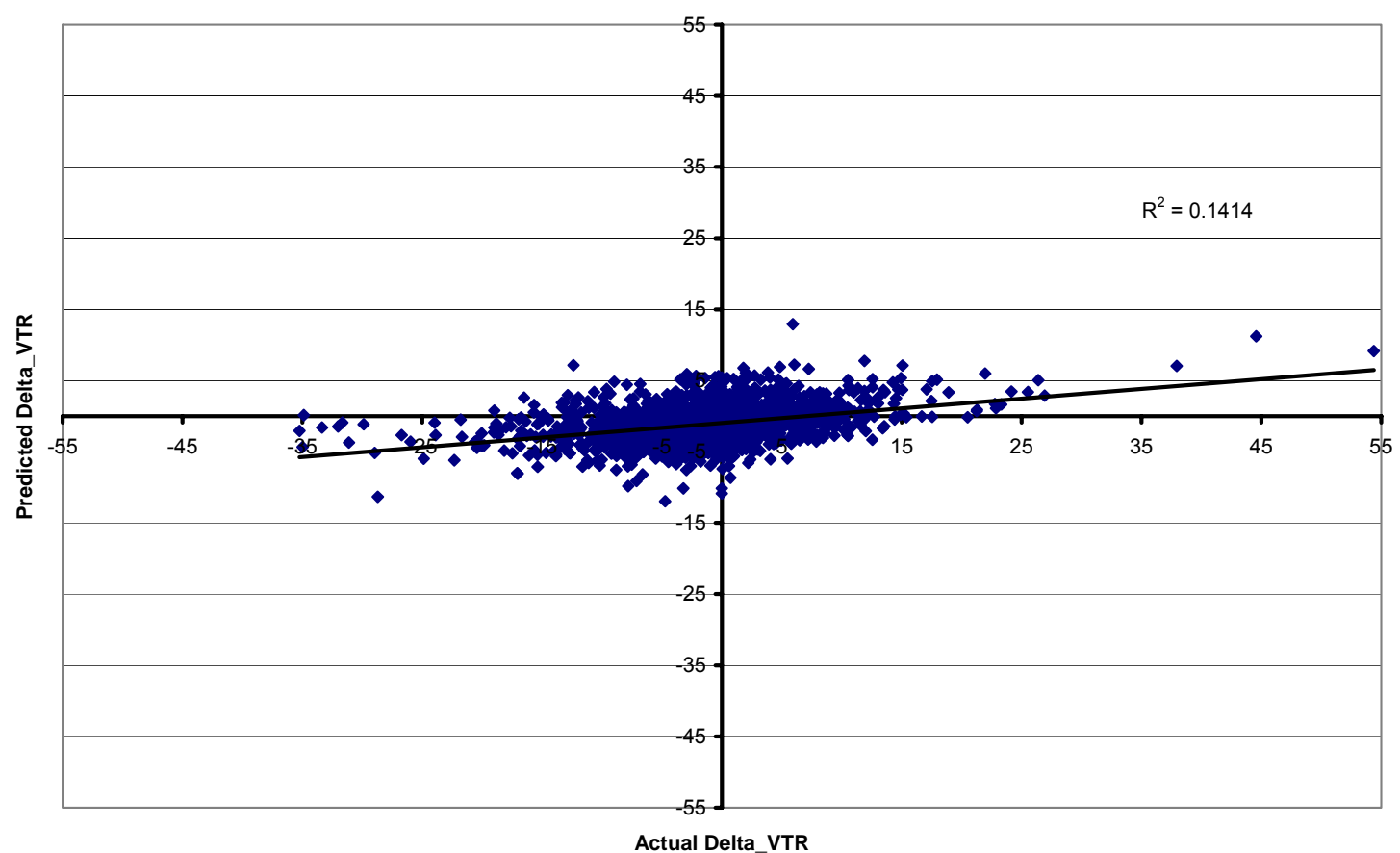

Figure 63: Scatter plot for Los Angeles validation set 
Predicted Delta_VTR Vs. Actual Delta_VTR

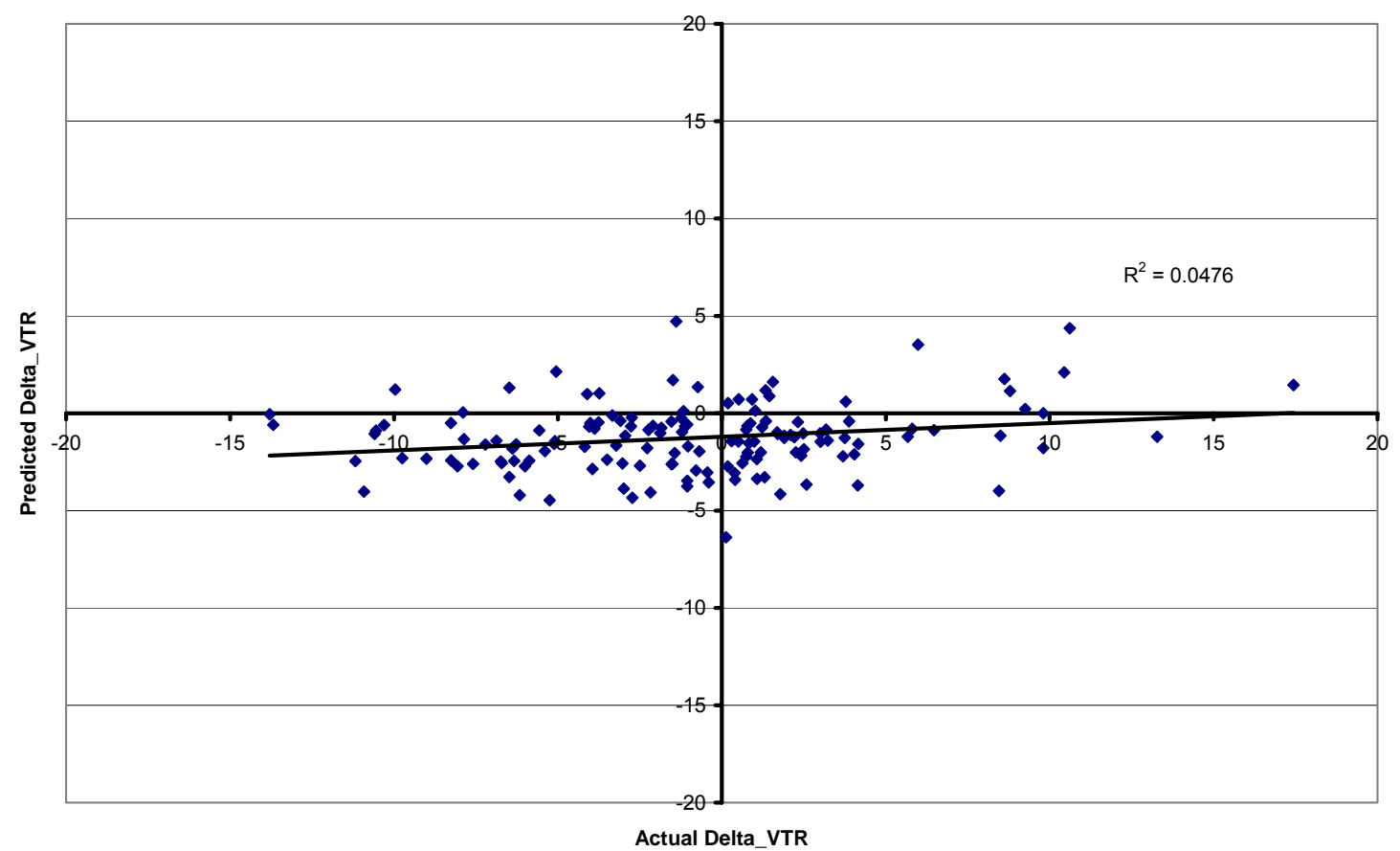

Figure 64: Scatter plot for Washington validation set

Actual Delta VTR Vs. Predicted Delta VTR

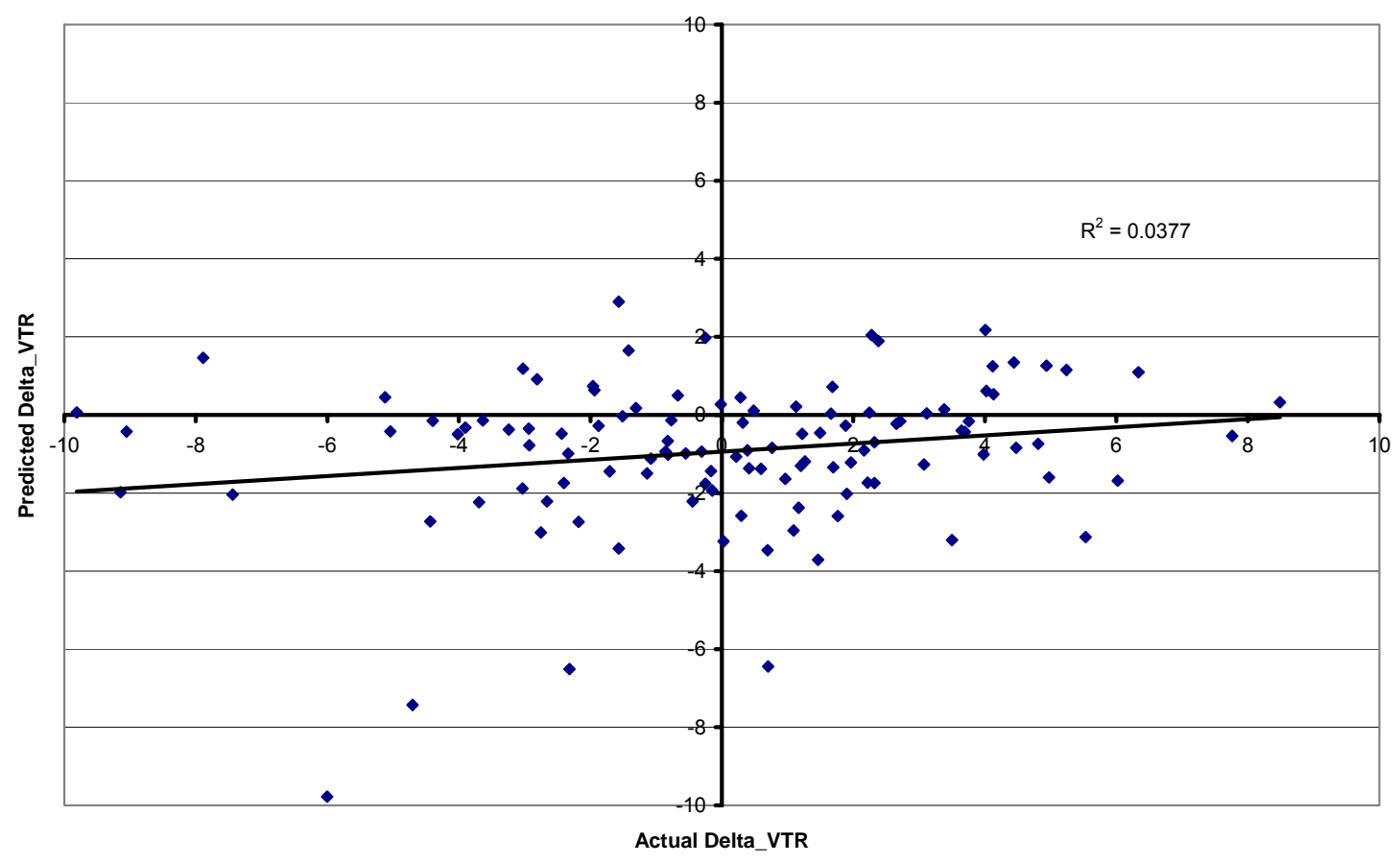

Figure 65: Scatter plot for Tucson validation set 
It can be concluded from these results that adding more information from other cities helped in improving the accuracy for Los Angles data, and so the neural network model built on this data is the recommended model for Los Angeles data. For Tucson data, the accuracy charts did not reveal much about the goodness of the neural net model built on the combined data, but from the scatter plot for Tucson validation set it can be seen that the combined data model was much biased at predicting negative changes in VTR, rendering it unsuitable. For the Washington data, adding information from the other cities proved disadvantageous. This result might be due to a very large share of training records coming from the Los Angeles data. 


\section{Phase II: Equally Sampled Combined Data}

To alleviate the problem that caused the unusual results for Tucson and Washington data on the non-equalized combined data, the training data from all of the three cities was combined in such a manner that an equal number $(2,018)$ of records from each data set contributed to the combined training data. The number of examples in each bin for three validation and training/testing sets are shown in Table 44.

Table 42: Equalized Combined Data - No. of Records in bins

\begin{tabular}{|l|c|c|c|c|c|c|c|c|c|}
\hline & Total & a1 & A2 & a3 & a4 & a5 & a6 & a7 & a8 \\
\hline Validation (LA) & $\mathbf{1 8 3 8}$ & 292 & 220 & 261 & 290 & 173 & 157 & 186 & 259 \\
Validation (Tucson) & $\mathbf{1 1 2}$ & 5 & 4 & 9 & 17 & 17 & 21 & 19 & 20 \\
Validation (wash) & $\mathbf{1 4 4}$ & 17 & 17 & 13 & 15 & 16 & 26 & 18 & 22 \\
Train +Test & $\mathbf{6 0 5 4}$ & 0 & 46 & 1371 & 3059 & 777 & 514 & 226 & 61 \\
\hline
\end{tabular}

A simple neural network with no variable selection and a forced enter linear regression model were built with the equally sampled training data. This combined model only contained 23 variables, which are shown in Table 42. The charts in Figures 66, 67, and 68 show the results of the comparison between the three performance measures for the combined, equally sampled models.

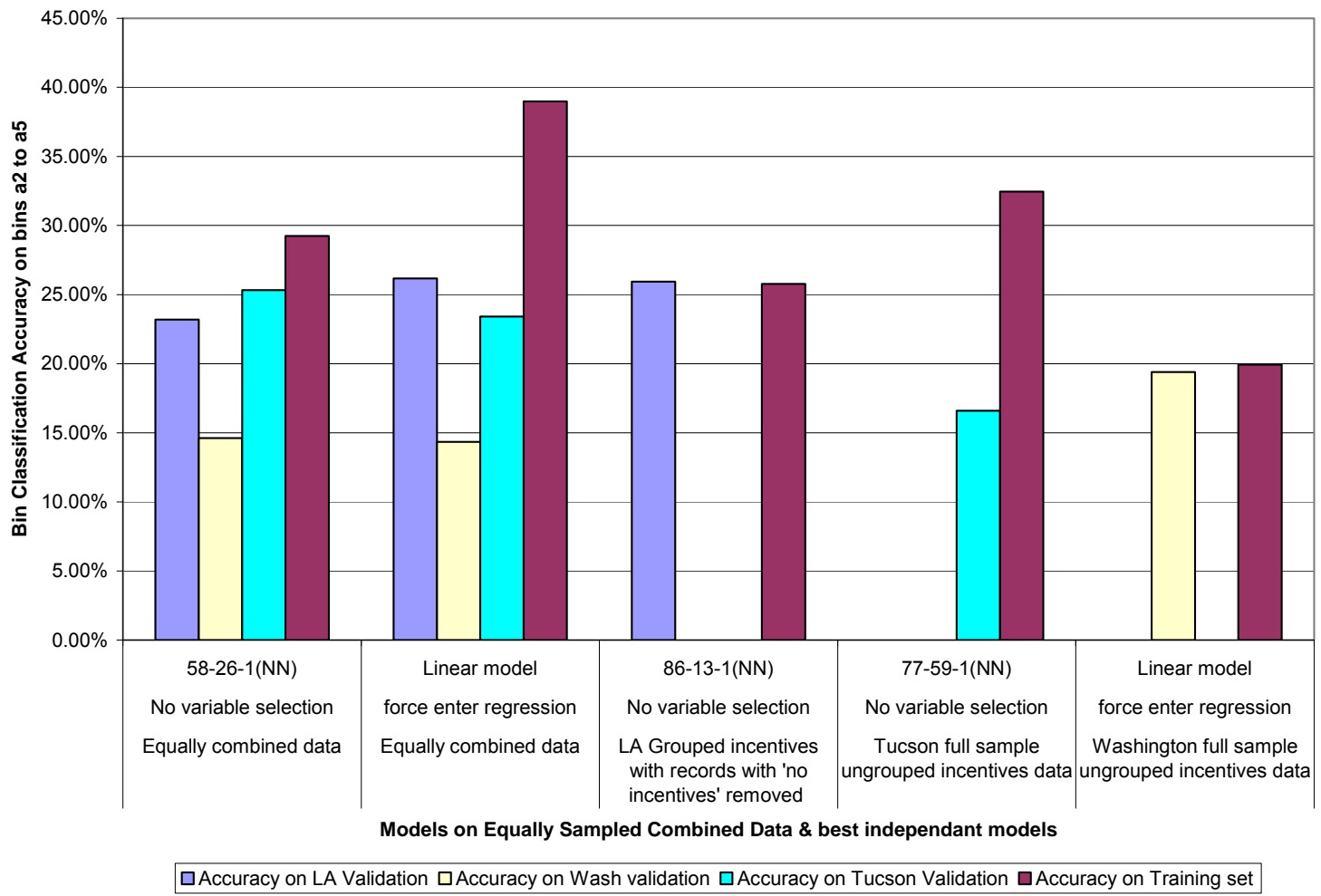

Figure 66: Bin Classification Accuracy on Moderate Range of change in VTR (a2 to a5) for three data sets (Models on equally combined data $\&$ best independent models) 


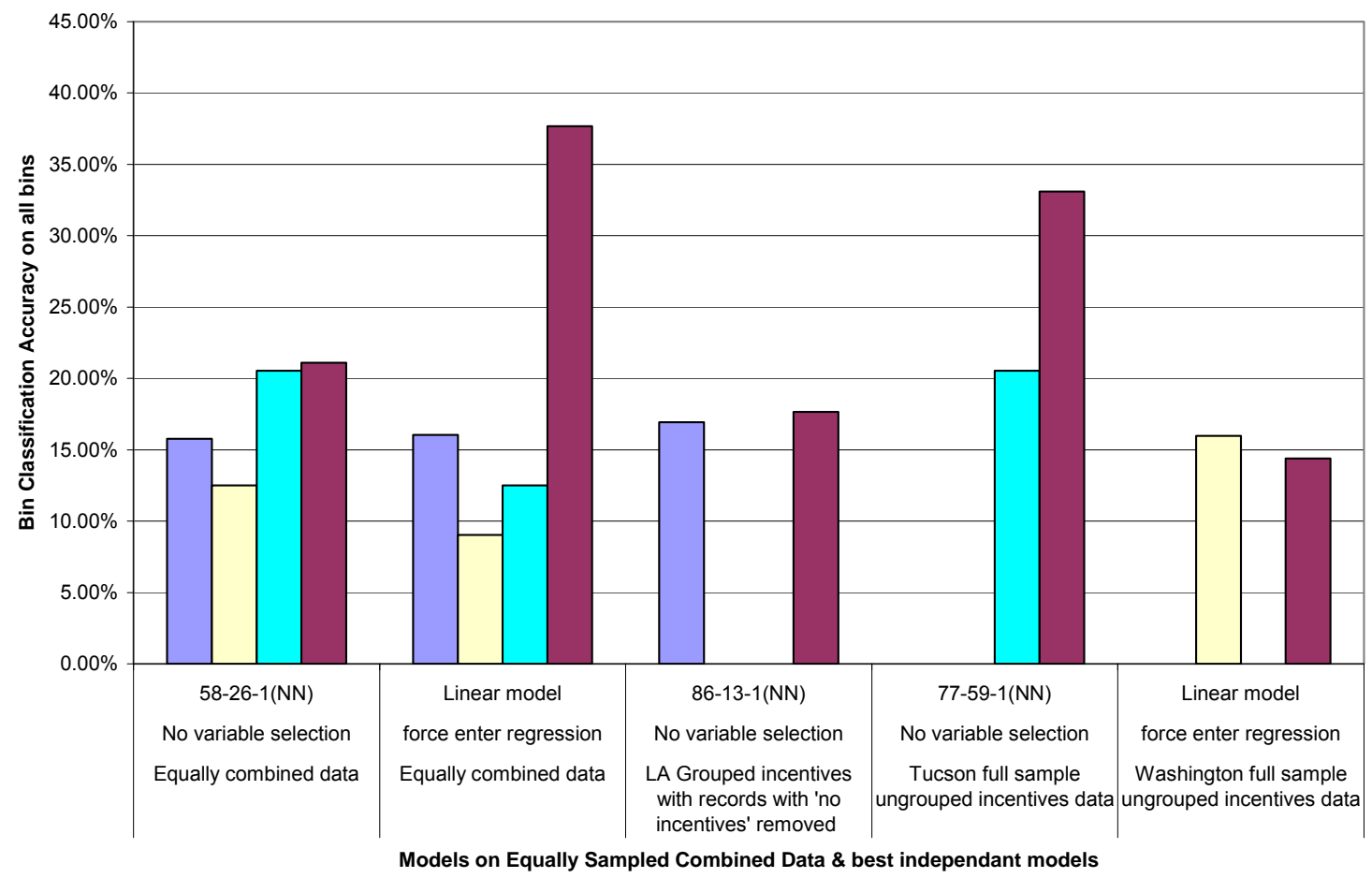

$\square$ Accuracy on LA Validation $\square$ Accuracy on Wash validation $\square$ Accuracy on Tucson Validation $\square$ Accuracy on Training set

Figure 67: Bin Classification Accuracy on Full Range of change in VTR (all bins) for three validation $\&$ training sets (Models on equally combined data \& best independent models)

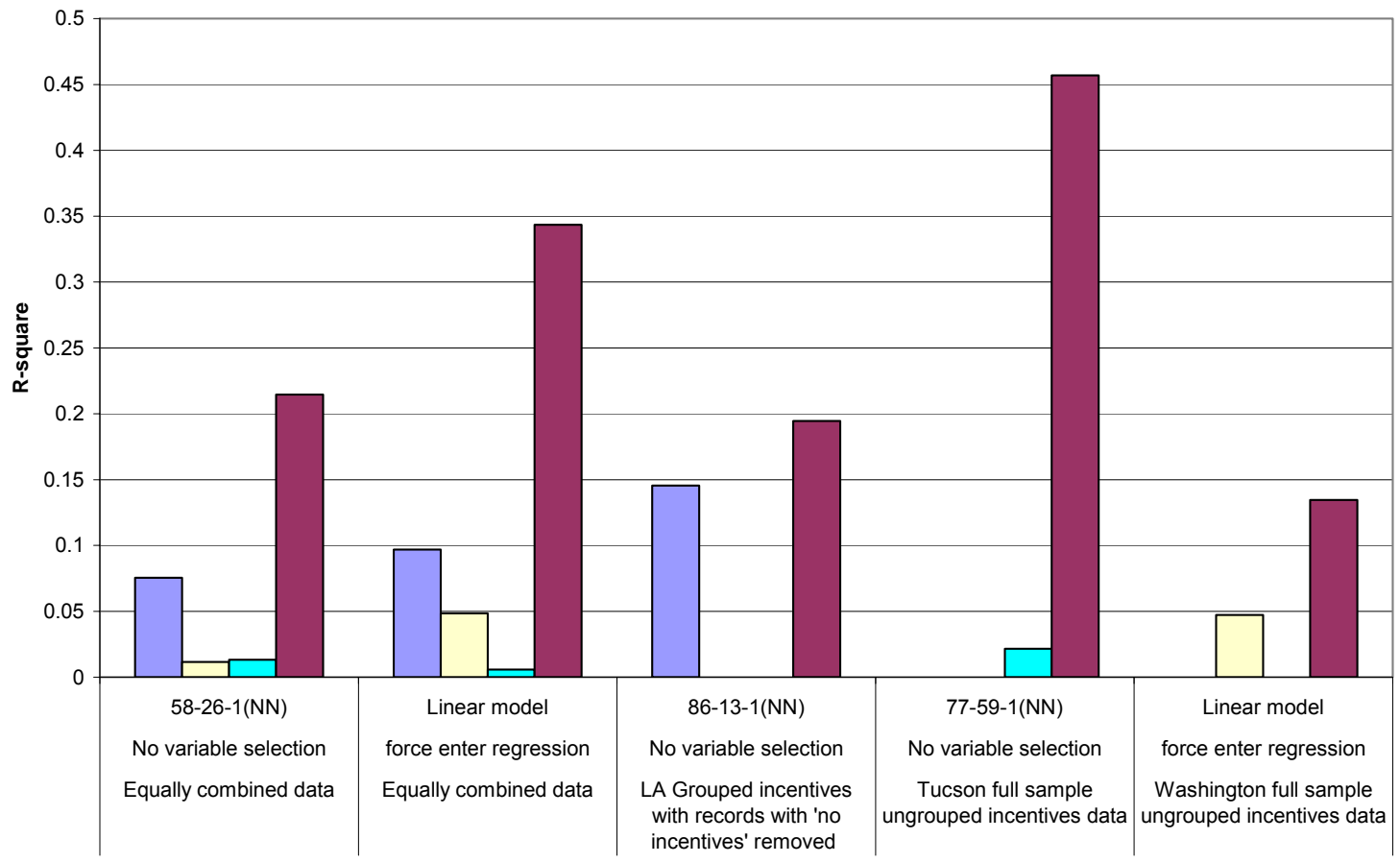

Models on Equally Sampled Combined Data \& best independant models

$\square R$-square on LA Validation $\square R$-square on Wash validation $\square R$-square on Tucson Validation $\square$ R-square on Training set

Figure 68: R-square for three validation \& training set (Models on equally combined data $\&$ best independent models) 
Figure 66 displays the comparison of the results between the neural net model with no variable selection and the forced enter regression model built on the equally sampled combined data and the recommended independent models on three datasets. Both combined data models were able to get better 'bin classification accuracy on moderate range of change in VTR' on Tucson validation set (NN model: 25.32 percent and Linear model: 23.40 percent) as compared to the recommended independent Tucson model (16.6 percent) but were not able to improve any accuracy on Los Angeles and Washington validation sets.

From Figure 67, it can be seen that neural net model built on the equally sampled combined data with no variable selection was able to get same 'bin classification accuracy on full range of change in VTR' on Tucson validation set (20.54 percent) as the recommended independent Tucson model. No other significant improvements were obtained by these combined data models over other recommended independent models. The same was true for R-squares values.

The scatter plots for the neural net model built on combined data on three validation sets are shown in Figures 69, 70, and 71.

Actual Delta_VTR Vs. Predicted Delta_VTR

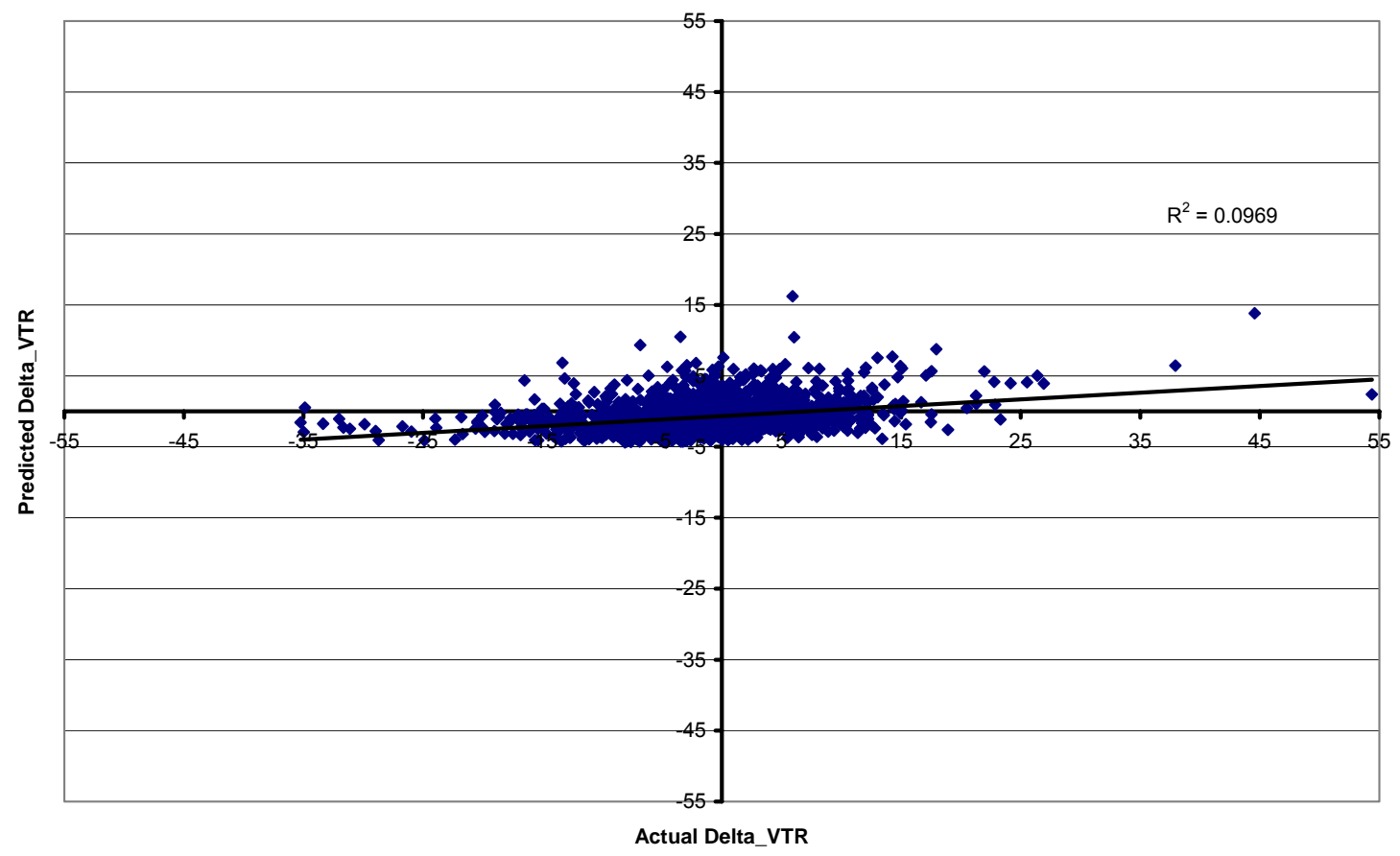

Figure 69: Scatter plot for Los Angeles validation set 
Predicted Delta_VTR Vs. Actual Delta_VTR

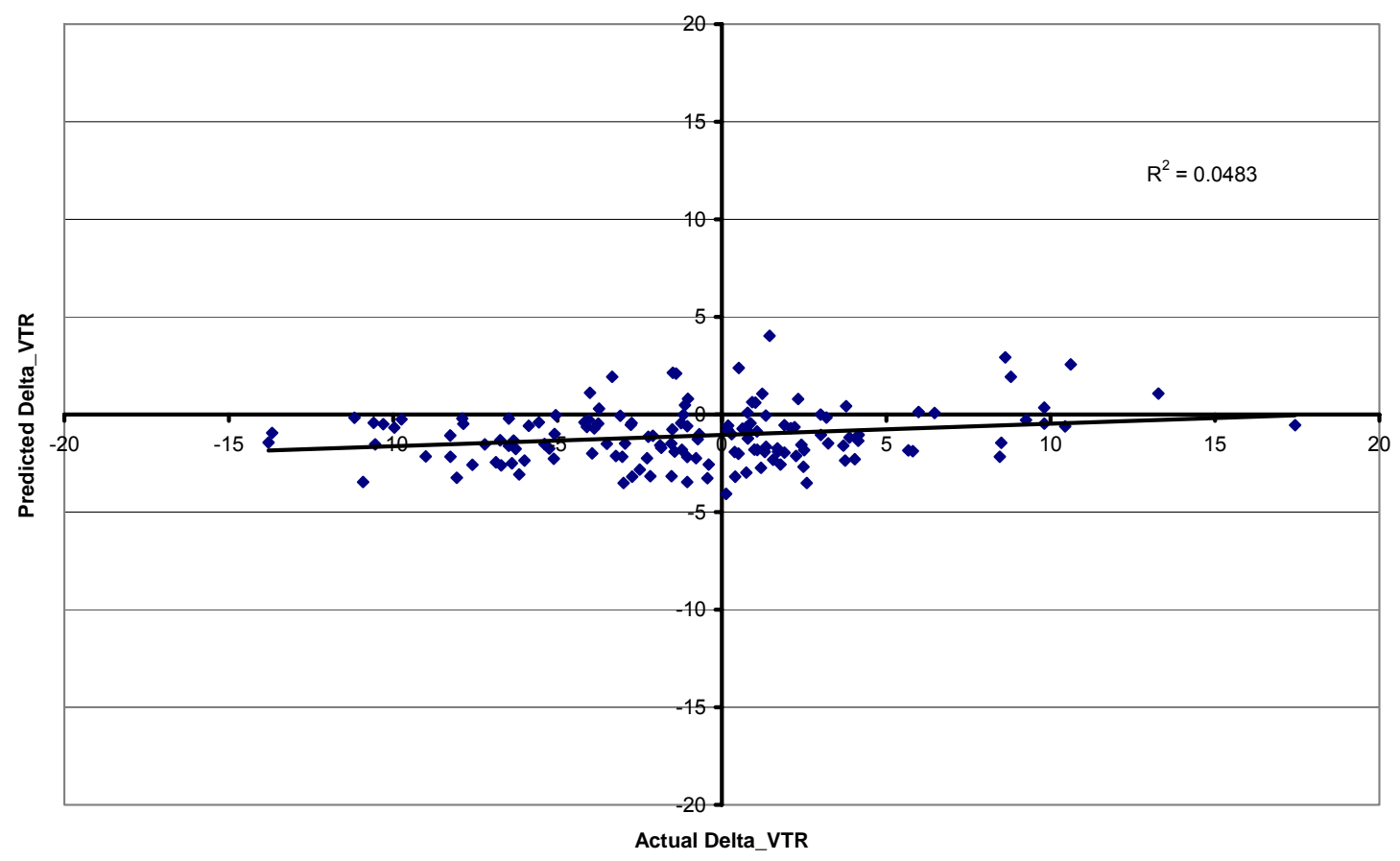

Figure 70: Scatter plot for Washington validation set

Predicted Delta_VTR Vs. Actual Delta_VTR

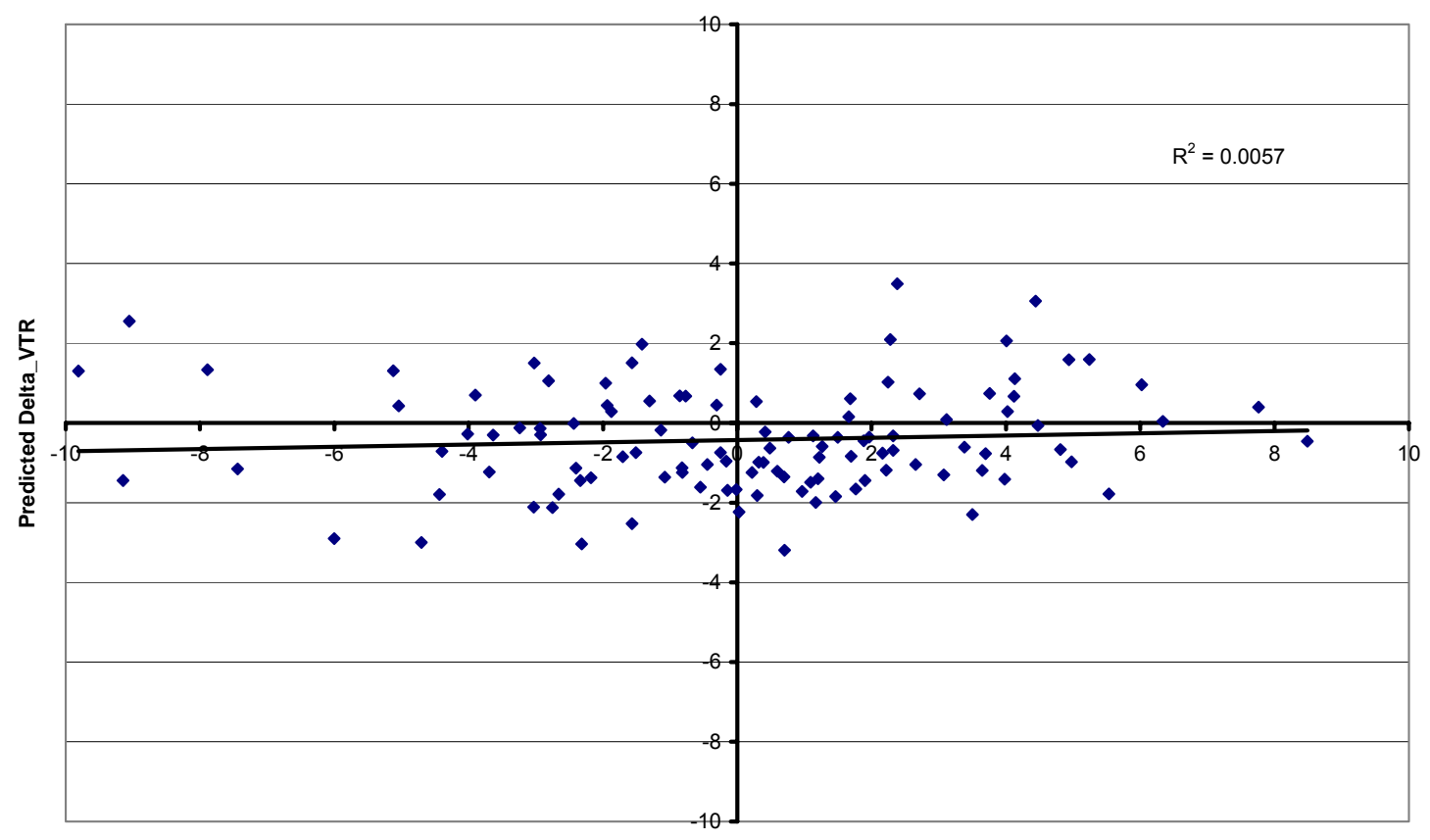

Actual Delta_VTR

Figure 71: Scatter plot for Tucson validation set 


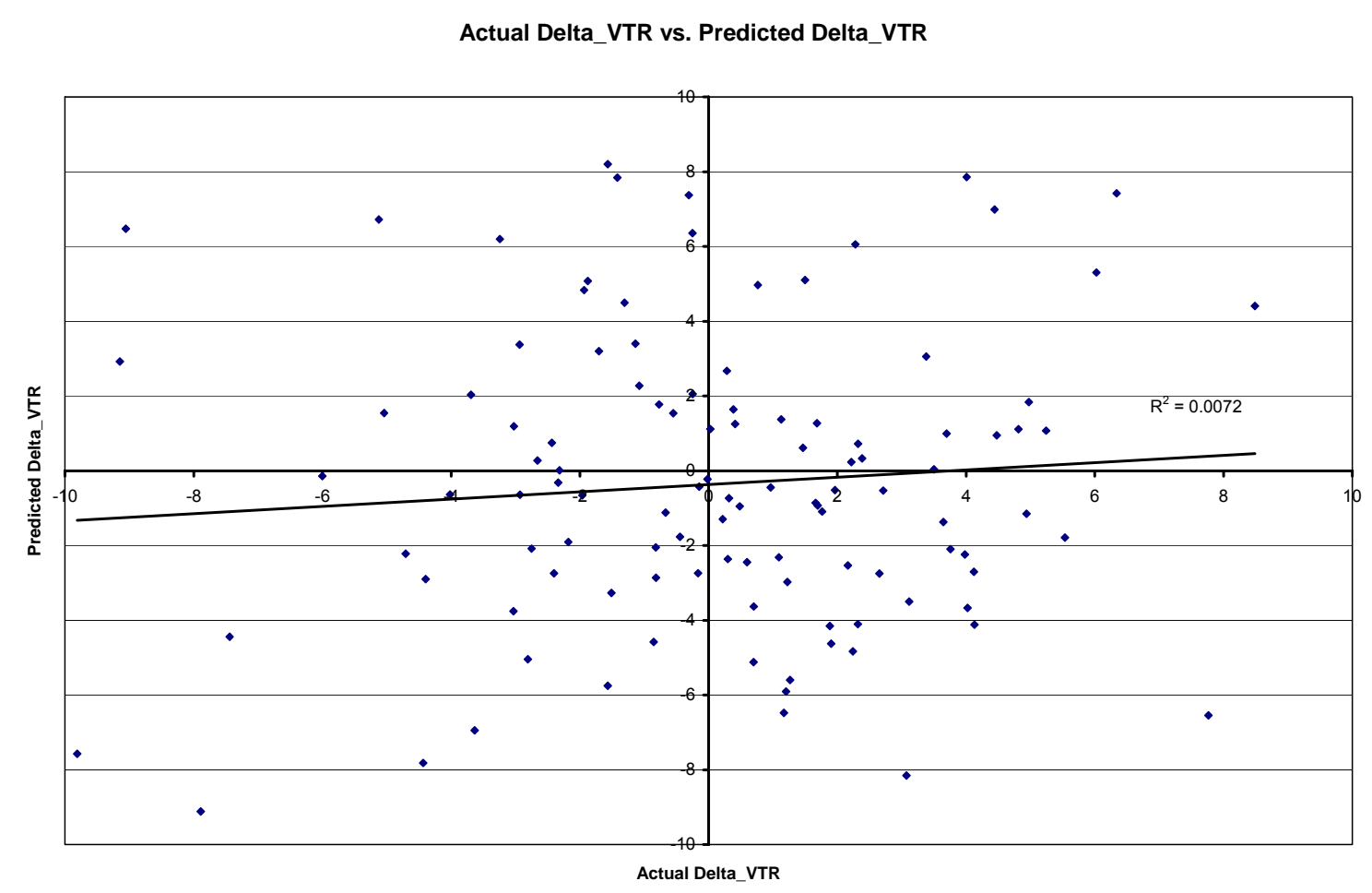

Figure 72: Scatter plot for neural network model built on over-sampled grouped Tucson data (recommended Tucson model)

Comparing the bin accuracy and R-square value charts, a conclusion was reached that equally sampled combined models were not able to improve the accuracy of the Los Angles and Washington data sets. The reason for such poor accuracy with the Los Angeles data may be due to such a small representation of Los Angeles data. Only 2,018 samples of the training data set were from Los Angeles. Inconsistency between Washington and the other two data sets could also be held responsible for the poor accuracy between Washington and the other data sets.

For the Tucson data, the accuracy in moderate range of change in VTR was improved with full range accuracy remaining constant at cost of reduction in the R-square as compared to the recommended model. But when examining the scatter plot, the neural net model that was built on equally sampled combined data only predicted small changes in VTR as compared to the recommended Tucson model, which predicted large changes in VTR. It was very difficult to decide between these two models and choose one as the best. Just like in the Washington data, when it was impossible to pick the best model, a cross-tab for positive/negative actual changes in VTR against positive/negative predicted changes in VTR on validation set was done. 
Table 43 A-D: Validation Set

A

\begin{tabular}{|c|c|c|}
\hline \multicolumn{3}{|c|}{ NN model on full sample ungrouped } \\
incentive dataset \\
\hline $\begin{array}{c}\text { Actual Delta_VTR / } \\
\text { Predicted Delta_VTR }\end{array}$ & Negative & Positive \\
\hline Negative & 25 & 31 \\
\hline Positive & 27 & 29 \\
\hline
\end{tabular}

\section{C}

\begin{tabular}{|c|c|}
\hline \multicolumn{2}{|c|}{$\begin{array}{c}\text { NN model on full sample ungrouped } \\
\text { incentive dataset }\end{array}$} \\
\hline precision & 0.446 \\
\hline recall & 0.481 \\
\hline F-value & 0.463 \\
\hline
\end{tabular}

B

\begin{tabular}{|c|c|c|}
\hline \multicolumn{3}{|c|}{$\begin{array}{l}\text { NN model on equally sampled combined } \\
\text { data }\end{array}$} \\
\hline $\begin{array}{l}\text { Actual Delta_VTR / } \\
\text { Predicted Delta VTR }\end{array}$ & Negative & Positive \\
\hline Negative & 34 & 18 \\
\hline Positive & 41 & 19 \\
\hline
\end{tabular}

D

\begin{tabular}{|l|c|}
\hline \multicolumn{2}{|c|}{ NN model on equally sampled } \\
combined data
\end{tabular}

From the validation set cross-tabulations, it was decided that the neural network model that was built on equally sampled combined data was better than the independent model built on the over-sampled grouped incentives. The neural network model built on the equally sampled combined data resulted in better $\mathrm{F}$-value on the validation set and therefore becomes the recommended model for Tucson. 


\section{WORKSITE TRI P RedUCtION MANUAL}

\section{Manual for Combined Data Model (General Model)}

The only model to get better results simultaneously on all three cities' validation sets was a neural network model built with no variable selection on equally sampled combined data. For this model, the training data from all of the three cities was combined in such a manner that an equal number $(2,018)$ of records from each training data set contributed to the combined training data, thus eliminating the bias towards any dataset. The variables in this equally sampled combined dataset are shown in Table 46.

Table 44: Combined data variables and grouping

\begin{tabular}{|c|c|c|}
\hline Variables & Description & Grouping \\
\hline ALONESHARE & Alone Share & \\
\hline TRANSITSHARE & Transit Share & \\
\hline CVPOOLSHARE & Carpool +Vanpool Share & \\
\hline WALKSHARE & Walk Share & \\
\hline BCYCLESHARE & Bicycle Share & \\
\hline MCYCLESHARE & Motorcycle Share & \\
\hline TELESHARE & Telecommute Share & \\
\hline CWW336 & 3/36 compressed work week share & \\
\hline CWW440 & 4/40 compressed work week share & \\
\hline CWW980 & 8/80 compressed work week share & \\
\hline FACILITY_AMENITIES & facilities \& amenities & $\begin{array}{l}\text { Passenger Loading Areas } \\
\text { Other Facility Improvements } \\
\text { Preferential Parking Areas } \\
\text { Bike Racks and Bike Lockers } \\
\text { Shower and Lockers } \\
\end{array}$ \\
\hline GRH & Guaranteed ride home programs & $\begin{array}{l}\text { TMA/TMO Provided Guaranteed Return Trip } \\
\text { Company Vehicle Guaranteed Return Trip } \\
\text { Emergencies Guaranteed Return Trip } \\
\text { Other Guaranteed Return Trip Program } \\
\text { Rental Car Guaranteed Return Trip } \\
\text { Taxi Guaranteed Return Trip } \\
\text { Unscheduled Overtime Guaranteed Return }\end{array}$ \\
\hline FLEX & flexible timing & $\begin{array}{l}\text { Flextime for Ride sharers (Work Shifts) } \\
\text { Flextime for Ride sharers (Grace Period) }\end{array}$ \\
\hline MRKT & Marketing programs & $\begin{array}{l}\text { Commuter Information Center } \\
\text { Commuter Fairs } \\
\text { Focus Groups } \\
\text { Posted Materials } \\
\text { New Hire Orientation } \\
\text { Other Marketing Elements } \\
\text { Personal Communication } \\
\text { Company Recognition } \\
\text { Special Interest Club (Biking, Walking) } \\
\text { TMA/TMO Membership } \\
\text { Written Materials } \\
\text { Zip Code Meetings }\end{array}$ \\
\hline RS_MATCH & Ride share matching programs & $\begin{array}{l}\text { Regional Commuter Management Agency } \\
\text { Employer-Based Rideshare Matching System }\end{array}$ \\
\hline FINANCIAL & financial incentives & $\begin{array}{l}\text { Transportation Allowances } \\
\text { On-Going Bike-to-Work Subsidies }\end{array}$ \\
\hline
\end{tabular}




\begin{tabular}{|c|c|c|}
\hline & & $\begin{array}{l}\text { On-Going Carpooling Subsidies } \\
\text { Other Direct Financial Subsidies } \\
\text { On-Going Walk-to-Work Subsidies }\end{array}$ \\
\hline PARKMGT & Parking management & $\begin{array}{l}\text { Increased Parking Costs for Drive Alones } \\
\text { Other Parking Management Strategies } \\
\text { Subsidized Parking for Ride sharers } \\
\end{array}$ \\
\hline TELE & Telecommute program & $\begin{array}{l}\text { Work at Home } \\
\text { Work at Satellite Center }\end{array}$ \\
\hline CWW & Compressed work week program & $3 / 36,4 / 40,9 / 80$ \& other Compressed Work Week Schedule \\
\hline ONSITE & onsite incentives & $\begin{array}{l}\text { On-Site Childcare Service } \\
\text { Other On-Site Services } \\
\text { Cafeteria, ATM's, Postal, Fitness Center } \\
\text { Transit Information or Pass Sales } \\
\end{array}$ \\
\hline DIRECT_NONFINAN & Non financial incentives & $\begin{array}{l}\text { Auto Services (Fuel, Oil, Tune-Up) } \\
\text { Gift Certificates } \\
\text { Free Meals } \\
\text { Other Direct Non-Financial Incentives } \\
\text { Catalogue Points } \\
\text { Additional Time Off with Pay } \\
\text { Drawings, Free Meals, Certificates, etc }\end{array}$ \\
\hline COMMTAX & commuter tax benefit incentives & $\begin{array}{l}\text { Introductory Transit Passes or Subsidies } \\
\text { Subsidized Vanpool Seats } \\
\text { On-Going Transit Subsidies } \\
\text { On-Going Vanpooling Subsidies } \\
\end{array}$ \\
\hline VTR & Vehicle trip rate & Vehicle trip rate \\
\hline DELTA_VTR & Change in VTR (dependant variable) & Change in VTR (dependant variable) \\
\hline
\end{tabular}

For the purpose of creating a manual, the training data and the three city validation sets along with the unused training data from Los Angeles were combined into one file with 21,267 records. The neural network model with no variable selection built on equally sampled combined data was used to predict the change in Vehicle Trip Rate (VTR) for all of these records.

The Figures 73 and 74 show the number of records per Incentive plan combination and cumulative number of records versus distinct Incentive plans. 
Number of records per specific Incentive Plans combination Vs. Distinct Incentive plan combinations

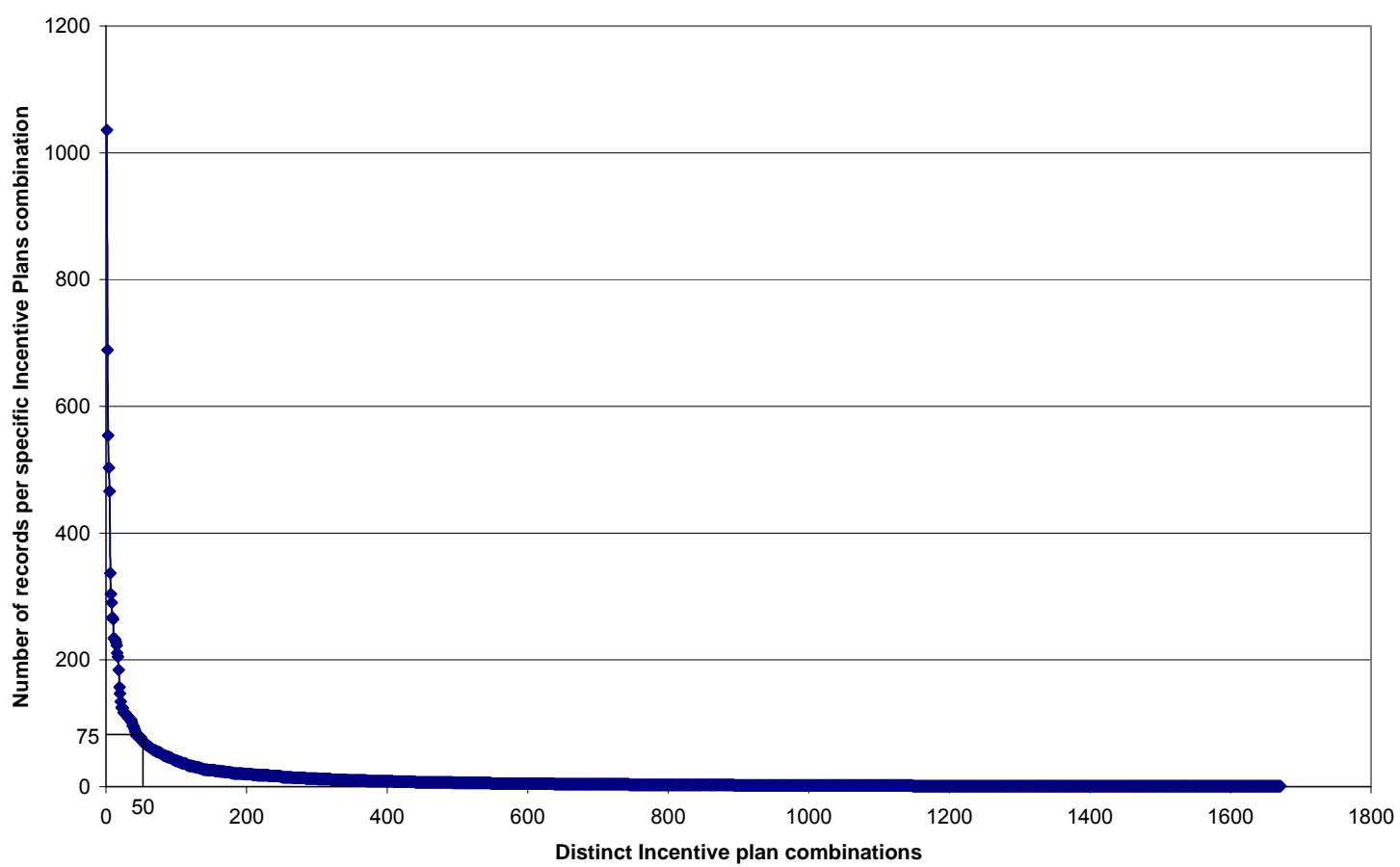

Figure 73: Number of records by incentive plan combinations

Number of records (cumulative) Vs. Distinct Incentive plan combinations

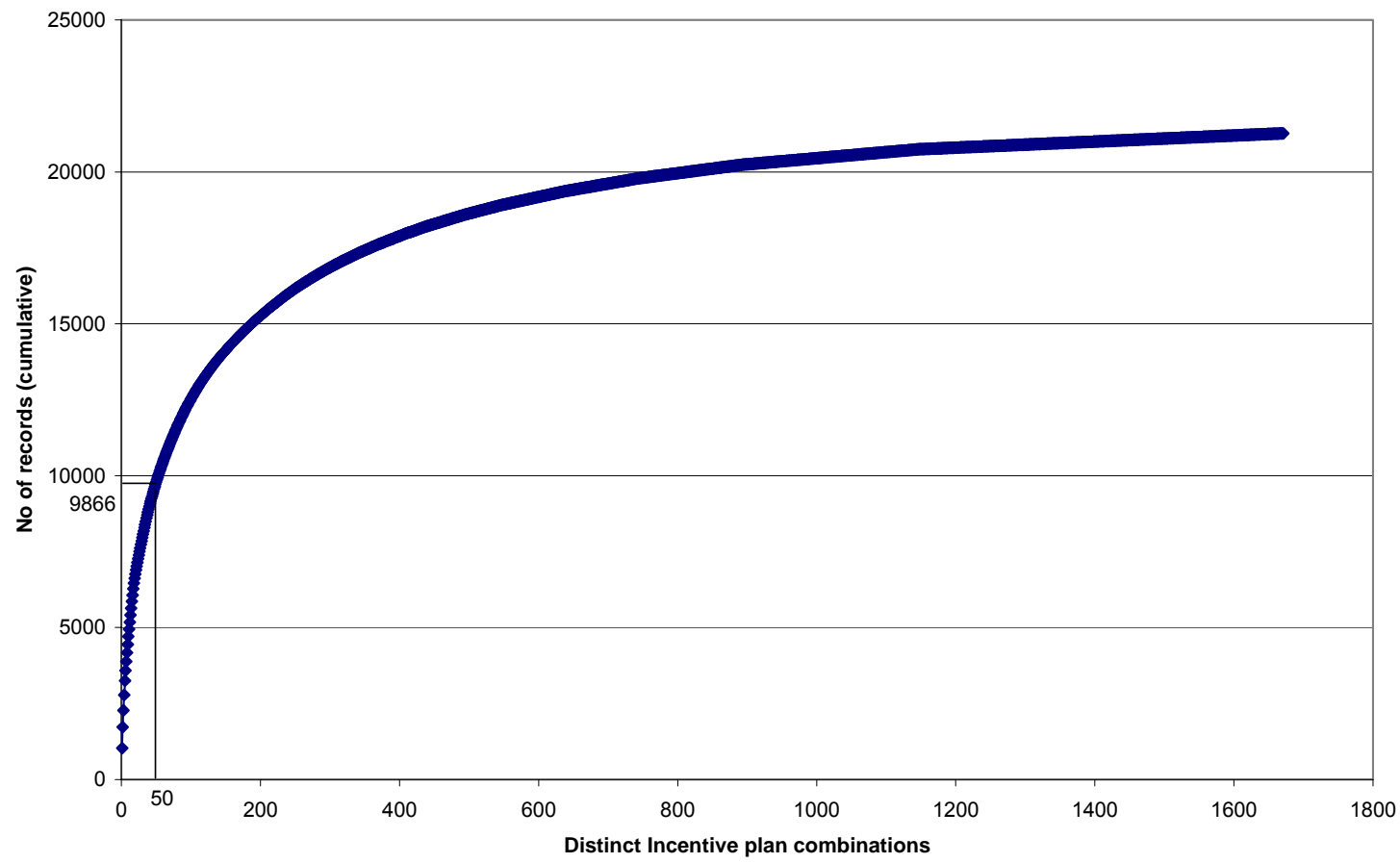

Figure 74: Number of records (cumulative) by plan combinations 
It can be seen from Figures 74 and 75 that there are 1,671 distinct incentive plan combinations in total, and out of these, 50 combinations are implemented by at least 75 records. And these 50 distinct Incentive plan combinations have been implemented by 9,866 records in total. These are the incentive plan combinations that seem to be widely accepted and implemented by many worksites. Table 46 shows these 50 incentive plan combinations and the total number of records implementing each specific combination ("1" indicates plan offered).

Table 45: Widely implemented incentive plan combinations

\begin{tabular}{|c|c|c|c|c|c|c|c|c|c|c|c|c|c|}
\hline Sr.No. & FACILITY_AMENITIES & onsite & commtax & FINANCIAL & CWw & FLEX & TELE & PARKMGT & direct_nonfinan & GRH & RS_MATCH & Mrkt & Total \\
\hline 1 & $\sqrt{ }$ & $\sqrt{ }$ & $\sqrt{ }$ & $\sqrt{ }$ & & & & & $\sqrt{ }$ & $\sqrt{ }$ & $\sqrt{ }$ & $\sqrt{ }$ & \begin{tabular}{|l|l}
1036 \\
\end{tabular} \\
\hline 2 & $\sqrt{ }$ & $\sqrt{ }$ & $\sqrt{ }$ & $\sqrt{ }$ & & $\sqrt{ }$ & & & $\sqrt{ }$ & $\sqrt{ }$ & $\sqrt{ }$ & $\sqrt{ }$ & 689 \\
\hline 3 & $\sqrt{ }$ & $\sqrt{ }$ & $\sqrt{ }$ & & & & & & $\sqrt{ }$ & $\sqrt{ }$ & $\sqrt{ }$ & $\sqrt{ }$ & 554 \\
\hline 4 & $\sqrt{ }$ & & $\sqrt{ }$ & $\sqrt{ }$ & & & & & $\sqrt{ }$ & $\sqrt{ }$ & $\sqrt{ }$ & $\sqrt{ }$ & 503 \\
\hline 5 & $\sqrt{ }$ & $\sqrt{ }$ & & & & & & & $\sqrt{ }$ & $\sqrt{ }$ & $\sqrt{ }$ & $\checkmark$ & 466 \\
\hline 6 & & $\sqrt{ }$ & $\sqrt{ }$ & $\sqrt{ }$ & & & & & $\sqrt{ }$ & $\sqrt{ }$ & $\sqrt{ }$ & $\sqrt{ }$ & 337 \\
\hline 7 & $\checkmark$ & $\sqrt{ }$ & & & & $\sqrt{ }$ & & & $\sqrt{ }$ & $\sqrt{ }$ & $\sqrt{ }$ & $\sqrt{ }$ & 304 \\
\hline 8 & $\sqrt{ }$ & $\sqrt{ }$ & $\checkmark$ & $\sqrt{ }$ & $\sqrt{ }$ & & & & $\sqrt{ }$ & $\sqrt{ }$ & $\sqrt{ }$ & $\checkmark$ & 290 \\
\hline 9 & $\sqrt{ }$ & & $\checkmark$ & & & & & & $\sqrt{ }$ & $\sqrt{ }$ & $\sqrt{ }$ & $\sqrt{ }$ & 267 \\
\hline 10 & $\sqrt{ }$ & $\sqrt{ }$ & & & $\sqrt{ }$ & & & & $\sqrt{ }$ & $\sqrt{ }$ & $\sqrt{ }$ & $\sqrt{ }$ & 264 \\
\hline 11 & $\sqrt{ }$ & & $\sqrt{ }$ & $\sqrt{ }$ & & & & & & $\sqrt{ }$ & $\sqrt{ }$ & $\sqrt{ }$ & 234 \\
\hline 12 & $\sqrt{ }$ & $-a_{0}$ & & & & & & & 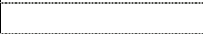 & $\sqrt{ }$ & $\sqrt{ }$ & $\sqrt{ }$ & 233 \\
\hline 13 & $\sqrt{ }$ & $\sqrt{ }$ & $\sqrt{ }$ & & & $\sqrt{ }$ & & & $\sqrt{ }$ & $\sqrt{ }$ & $\sqrt{ }$ & $\sqrt{ }$ & 232 \\
\hline 14 & $\checkmark$ & $\sqrt{ }$ & $\checkmark$ & $\sqrt{ }$ & & & & & & $\sqrt{ }$ & $\sqrt{ }$ & $\sqrt{ }$ & 228 \\
\hline 15 & $\sqrt{ }$ & & & & & & & & $\sqrt{ }$ & $\sqrt{ }$ & $\sqrt{ }$ & $\sqrt{ }$ & 223 \\
\hline 16 & $\sqrt{ }$ & $\sqrt{ }$ & $\sqrt{ }$ & $\sqrt{ }$ & $\sqrt{ }$ & $\sqrt{ }$ & & & $\sqrt{ }$ & $\sqrt{ }$ & $\sqrt{ }$ & $\sqrt{ }$ & 211 \\
\hline 17 & $\sqrt{ }$ & $\sqrt{ }$ & $\sqrt{ }$ & $\sqrt{ }$ & & & & & $\sqrt{ }$ & $\sqrt{ }$ & & $\sqrt{ }$ & 205 \\
\hline 18 & $\sqrt{ }$ & $\sqrt{ }$ & $\sqrt{ }$ & & $\sqrt{ }$ & & & & $\sqrt{ }$ & $\sqrt{ }$ & $\sqrt{ }$ & $\sqrt{ }$ & 184 \\
\hline 19 & $\sqrt{ }$ & & $\sqrt{ }$ & $\sqrt{ }$ & & $\sqrt{ }$ & & & $\sqrt{ }$ & $\sqrt{ }$ & $\sqrt{ }$ & $\sqrt{ }$ & 157 \\
\hline 20 & & & $\sqrt{ }$ & $\sqrt{ }$ & & & & & $\sqrt{ }$ & $\sqrt{ }$ & $\sqrt{ }$ & $\sqrt{ }$ & 147 \\
\hline 21 & $\sqrt{ }$ & $\sqrt{ }$ & & & $\sqrt{ }$ & & $\sqrt{ }$ & & $\sqrt{ }$ & $\sqrt{ }$ & $\sqrt{ }$ & $\sqrt{ }$ & 134 \\
\hline 22 & $\sqrt{ }$ & .......... & $\sqrt{ }$ & & & $\sqrt{ }$ & & & V & V & $\sqrt{ }$ & $\sqrt{ }$ & 125 \\
\hline 23 & $\sqrt{ }$ & "am- & & & & & & & & & $\sqrt{ }$ & $\sqrt{ }$ & 124 \\
\hline 24 & $\sqrt{ }$ & $\sqrt{ }$ & & $\sqrt{ }$ & & & & & $\sqrt{ }$ & $\sqrt{ }$ & $\sqrt{ }$ & $\sqrt{ }$ & 124 \\
\hline 25 & $\sqrt{ }$ & $\sqrt{ }$ & $\checkmark$ & & $\sqrt{ }$ & $\sqrt{ }$ & & & $\sqrt{ }$ & $\sqrt{ }$ & $\sqrt{ }$ & $\sqrt{ }$ & 117 \\
\hline 26 & $\sqrt{ }$ & $\sqrt{ }$ & $\sqrt{ }$ & $\sqrt{ }$ & & $\sqrt{ }$ & & & & $\sqrt{ }$ & $\sqrt{ }$ & $\sqrt{ }$ & 117 \\
\hline 27 & $\sqrt{ }$ & $\sqrt{ }$ & & & & & & & & & $\sqrt{ }$ & $\sqrt{ }$ & 116 \\
\hline 28 & $\sqrt{ }$ & $\sqrt{ }$ & $\sqrt{ }$ & & $\sqrt{ }$ & & $\sqrt{ }$ & & $\sqrt{ }$ & $\checkmark$ & $\sqrt{ }$ & $\sqrt{ }$ & 115 \\
\hline 29 & $\sqrt{ }$ & $\sqrt{ }$ & $\sqrt{ }$ & & & & & & $\sqrt{ }$ & $\sqrt{ }$ & & $\sqrt{ }$ & 113 \\
\hline 30 & $\sqrt{ }$ & & $\sqrt{ }$ & $\sqrt{ }$ & $\sqrt{ }$ & & & & $\sqrt{ }$ & $\sqrt{ }$ & $\sqrt{ }$ & $\sqrt{ }$ & 111 \\
\hline 31 & $\sqrt{ }$ & $\sqrt{ }$ & & & & & & & & $\sqrt{ }$ & $\sqrt{ }$ & $\sqrt{ }$ & 111 \\
\hline 32 & $\sqrt{ }$ & & & & & $\sqrt{ }$ & & & $\sqrt{ }$ & $\sqrt{ }$ & $\sqrt{ }$ & $\sqrt{ }$ & 108 \\
\hline 33 & & $\sqrt{ }$ & $\sqrt{ }$ & & & & & & $\sqrt{ }$ & $\sqrt{ }$ & $\sqrt{ }$ & $\sqrt{ }$ & 107 \\
\hline 34 & $\sqrt{ }$ & $\sqrt{ }$ & & $\sqrt{ }$ & $\sqrt{ }$ & $\sqrt{ }$ & & & $\sqrt{ }$ & $\sqrt{ }$ & $\sqrt{ }$ & $\sqrt{ }$ & 106 \\
\hline 35 & $\sqrt{ }$ & $\sqrt{ }$ & $\sqrt{ }$ & & & & & & & $\sqrt{ }$ & $\sqrt{ }$ & $\sqrt{ }$ & 106 \\
\hline 36 & $\sqrt{ }$ & & $\sqrt{ }$ & $\sqrt{ }$ & & & & & $\sqrt{ }$ & $\sqrt{ }$ & & $\sqrt{ }$ & 105 \\
\hline 37 & $\sqrt{ }$ & & & $\checkmark$ & & & & & $\sqrt{ }$ & $\sqrt{ }$ & $\sqrt{ }$ & $\sqrt{ }$ & 101 \\
\hline 38 & $\sqrt{ }$ & $\sqrt{ }$ & $\sqrt{ }$ & $\sqrt{ }$ & $\sqrt{ }$ & & $\sqrt{ }$ & & $\sqrt{ }$ & $\sqrt{ }$ & $\sqrt{ }$ & $\checkmark$ & 96 \\
\hline 39 & $\sqrt{ }$ & $\sqrt{ }$ & $\sqrt{ }$ & $\sqrt{ }$ & $\sqrt{ }$ & $\sqrt{ }$ & $\sqrt{ }$ & & $\sqrt{ }$ & $\sqrt{ }$ & $\sqrt{ }$ & $\sqrt{ }$ & 96 \\
\hline 40 & V & & & $\checkmark$ & & & & & & $\sqrt{ }$ & & $\sqrt{ }$ & 92 \\
\hline 41 & $\sqrt{ }$ & $\sqrt{ }$ & & & & & & & $\sqrt{ }$ & & $\sqrt{ }$ & $\sqrt{ }$ & 89 \\
\hline 42 & $\sqrt{ }$ & $\sqrt{ }$ & & & $\sqrt{ }$ & & $\sqrt{ }$ & & & $\sqrt{ }$ & $\sqrt{ }$ & $\sqrt{ }$ & 86 \\
\hline 43 & $\sqrt{ }$ & $\sqrt{ }$ & & & $\sqrt{ }$ & & $\sqrt{ }$ & & $\sqrt{ }$ & & $\sqrt{ }$ & $\sqrt{ }$ & 81 \\
\hline 44 & $\sqrt{ }$ & $\sqrt{ }$ & $\sqrt{ }$ & $\sqrt{ }$ & & & $\sqrt{ }$ & & $\sqrt{ }$ & $\sqrt{ }$ & $\sqrt{ }$ & $\checkmark$ & 81 \\
\hline 45 & $\sqrt{ }$ & $\sqrt{ }$ & & & $\sqrt{ }$ & & & & & & $\sqrt{ }$ & $\checkmark$ & 80 \\
\hline 46 & $\sqrt{ }$ & & & & $\sqrt{ }$ & $\checkmark$ & & & & $\sqrt{ }$ & $\sqrt{ }$ & $\sqrt{ }$ & 78 \\
\hline 47 & $\sqrt{ }$ & & $\sqrt{ }$ & $\sqrt{ }$ & $\sqrt{ }$ & $\sqrt{ }$ & & & $\checkmark$ & $\sqrt{ }$ & $\sqrt{ }$ & $\checkmark$ & 78 \\
\hline 48 & $\sqrt{ }$ & $\sqrt{ }$ & & & & & $\sqrt{ }$ & & & & $\sqrt{ }$ & $\sqrt{ }$ & 78 \\
\hline 49 & $\sqrt{ }$ & $\sqrt{ }$ & $\sqrt{ }$ & $\sqrt{ }$ & & & & $\sqrt{ }$ & $\sqrt{ }$ & $\sqrt{ }$ & $\sqrt{ }$ & $\sqrt{ }$ & 78 \\
\hline 50 & & & J & & & & & & $\sqrt{ }$ & $\sqrt{ }$ & $\sqrt{ }$ & $\sqrt{ }$ & 76 \\
\hline
\end{tabular}


It can be seen from the table 46, that facilities \& Amenities (showers \& lockers, bike racks, etc.) and onsite incentives (onsite childcare, cafeteria, etc.) are dependant on infrastructure development were the worksite is located. So a transportation engineer using this manual can know it in advance if the new worksite under consideration has these incentives provided to their employees. Also from Transit infrastructure in the vicinity of the worksite, the baseline share of employees traveling by transit can be determined for the worksite.

So for example, if a new worksite expects its transit share to be in the range of $0 \%$ to $5 \%$ and has no knowledge about other shares, then assuming the other mode shares to be close to zero, the worksite might expect a vehicle trip rate in the range of $100 \%$ to $90 \%$. Our manual provides tables with these kinds of scenarios, in this case table 49, containing our learned models averaged change in VTR predictions, the averaged change in VTR observed at the worksites, and the number of worksite records that matched the transit share \& VTR scenario for the above stated incentive 50 incentive plan combinations. So for the above example, if the worksite offers all incentives except compressed work week, flexible timing, telecommuting and parking management incentives (Table 49, Sr.No.1), then according to our model the worksite can expect a reduction of 5.3 vehicle per 100 (i.e. -5.3 change in VTR), with 4.5 observed reduction over 238 records. The number of records can be used as a measure of confidence while using the predicted values. The all scenario tables in the manual use abbreviations for the names of the incentives as shown in table 48.

Table 46: Incentives codes

\begin{tabular}{|l|l|}
\hline Incentives & Code \\
\hline facilities \& amenities & F\&A \\
\hline Guaranteed ride home programs & GRH \\
\hline flexible timing & FLEX \\
\hline Marketing programs & MRKT \\
\hline Ride share matching programs & RSMP \\
\hline financial incentives & FIN \\
\hline Parking management & PMT \\
\hline Telecommute program & TELE \\
\hline Compressed work week program & CWW \\
\hline onsite incentives & ONS \\
\hline Non financial incentives & NONF \\
\hline commuter tax benefit incentives & CTB \\
\hline
\end{tabular}

\section{Procedure to look-up 'Change in VTR' prediction tables}

1) Decide your Transit share range (Infrastructure dependant)

2) Decide your Vehicle Trip Rate range

3) Find the appropriate table

4) Look-up the table for incentive plan combinations and its predicted change in VTR (bold), actual observed change in VTR and number of records matching that criteria 
Table 47: Model's change in VTR prediction for worksites with Transit share between $0 \%$ to $5 \%$ and Vehicle Trip Rate between 90 to 100

\begin{tabular}{|c|c|c|c|c|c|c|c|c|c|c|c|c|c|c|c|}
\hline $\begin{array}{l}\text { Sr. } \\
\text { No. }\end{array}$ & $F \& A$ & ONS & СТВ & FI N & CWW & FLEX & TELE & PMT & NONF & GRH & RSMP & MRKT & $\begin{array}{r}\text { Tran } \\
{[0} \\
\text { VTR- }\end{array}$ & $\begin{array}{l}\text { nsit Sha } \\
0-0.05) \\
{[100-9}\end{array}$ & \\
\hline & & & & & & & & & & & & & Predicted & Actual & No.Ex \\
\hline 1 & $\sqrt{ }$ & $\sqrt{ }$ & $\sqrt{ }$ & $\sqrt{ }$ & & & & & $\sqrt{ }$ & $\sqrt{ }$ & $\sqrt{ }$ & $\sqrt{ }$ & -5.3 & -4.5 & 238 \\
\hline 2 & $\sqrt{ }$ & $\sqrt{ }$ & $\sqrt{ }$ & $\sqrt{ }$ & & $\sqrt{ }$ & & & $\sqrt{ }$ & $\sqrt{ }$ & $\sqrt{ }$ & $\sqrt{ }$ & -3.7 & -4.9 & 162 \\
\hline 3 & $\sqrt{ }$ & $\sqrt{ }$ & $\sqrt{ }$ & & & & & & $\sqrt{ }$ & $\sqrt{ }$ & $\sqrt{ }$ & $\sqrt{ }$ & -6.5 & -3.9 & 120 \\
\hline 4 & $\sqrt{ }$ & & $\sqrt{ }$ & $\sqrt{ }$ & & & & & $\sqrt{ }$ & $\sqrt{ }$ & $\sqrt{ }$ & $\sqrt{ }$ & -6.1 & -3.8 & 123 \\
\hline 5 & $\sqrt{ }$ & $\sqrt{ }$ & & & & & & & $\sqrt{ }$ & $\sqrt{ }$ & $\sqrt{ }$ & $\sqrt{ }$ & -5.3 & -4.0 & 93 \\
\hline 6 & & $\sqrt{ }$ & $\sqrt{ }$ & $\sqrt{ }$ & & & & & $\sqrt{ }$ & $\sqrt{ }$ & $\sqrt{ }$ & $\sqrt{ }$ & -6.3 & -4.1 & 84 \\
\hline 7 & $\sqrt{ }$ & $\sqrt{ }$ & & & & $\sqrt{ }$ & & & $\sqrt{ }$ & $\checkmark$ & $\sqrt{ }$ & $\sqrt{ }$ & -4.1 & -3.2 & 81 \\
\hline 8 & $\sqrt{ }$ & $\sqrt{ }$ & $\sqrt{ }$ & $\sqrt{ }$ & $\sqrt{ }$ & & & & $\sqrt{ }$ & $\sqrt{ }$ & $\sqrt{ }$ & $\sqrt{ }$ & -2.8 & -4.6 & 30 \\
\hline 9 & $\sqrt{ }$ & & $\sqrt{ }$ & & & & & & $\sqrt{ }$ & $\sqrt{ }$ & $\sqrt{ }$ & $\sqrt{ }$ & -6.8 & -4.8 & 48 \\
\hline 10 & $\sqrt{ }$ & $\sqrt{ }$ & & & $\sqrt{ }$ & & & & $\sqrt{ }$ & $\sqrt{ }$ & $\sqrt{ }$ & $\sqrt{ }$ & -3.2 & -4.3 & 46 \\
\hline 11 & $\sqrt{ }$ & & $\sqrt{ }$ & $\checkmark$ & & & & & & $\sqrt{ }$ & $\sqrt{ }$ & $\sqrt{ }$ & -6.4 & -4.7 & 39 \\
\hline 12 & $\sqrt{ }$ & 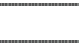 & & & & & & & & $\sqrt{ }$ & $\sqrt{ }$ & $\sqrt{ }$ & -6.9 & -1.4 & 62 \\
\hline 13 & $\sqrt{ }$ & $\sqrt{ }$ & $\sqrt{ }$ & & & $\sqrt{ }$ & & & $\sqrt{ }$ & $\sqrt{ }$ & $\sqrt{ }$ & $\sqrt{ }$ & -4.9 & -5.5 & 54 \\
\hline 14 & $\sqrt{ }$ & $\sqrt{ }$ & $\sqrt{ }$ & $\checkmark$ & & & & & & $\sqrt{ }$ & $\checkmark$ & $\sqrt{ }$ & -5.7 & -3.8 & 60 \\
\hline 15 & $\sqrt{ }$ & & & & & & & & $\sqrt{ }$ & $\sqrt{ }$ & $\sqrt{ }$ & $\sqrt{ }$ & -5.7 & -5.1 & 40 \\
\hline 16 & $\sqrt{ }$ & $\sqrt{ }$ & $\sqrt{ }$ & $\sqrt{ }$ & $\sqrt{ }$ & $\sqrt{ }$ & & & $\sqrt{ }$ & $\sqrt{ }$ & $\sqrt{ }$ & $\sqrt{ }$ & -1.5 & -8.0 & 38 \\
\hline 17 & $\sqrt{ }$ & $\sqrt{ }$ & $\sqrt{ }$ & $\sqrt{ }$ & & & & & $\sqrt{ }$ & $\sqrt{ }$ & & $\sqrt{ }$ & -5.8 & -4.4 & 42 \\
\hline 18 & $\sqrt{ }$ & $\sqrt{ }$ & $\checkmark$ & & $\checkmark$ & & & & $\sqrt{ }$ & $\checkmark$ & $\sqrt{ }$ & $\checkmark$ & -3.1 & -4.3 & 15 \\
\hline 19 & $\sqrt{ }$ & & $\sqrt{ }$ & $\sqrt{ }$ & & $\sqrt{ }$ & & & $\sqrt{ }$ & $\sqrt{ }$ & $\sqrt{ }$ & $\sqrt{ }$ & -4.3 & -3.9 & 49 \\
\hline 20 & & & $\sqrt{ }$ & $\sqrt{ }$ & & & & & $\sqrt{ }$ & $\sqrt{ }$ & $\sqrt{ }$ & $\sqrt{ }$ & -5.7 & -5.1 & 28 \\
\hline 21 & $\sqrt{ }$ & $\sqrt{ }$ & & & V & & $\sqrt{ }$ & & $\sqrt{ }$ & $\sqrt{ }$ & $\sqrt{ }$ & $\checkmark$ & -3.2 & -7.3 & 16 \\
\hline 22 & $\sqrt{ }$ & & $\sqrt{ }$ & & & $\sqrt{ }$ & & & $\sqrt{ }$ & $\sqrt{ }$ & $\sqrt{ }$ & $\sqrt{ }$ & -5.5 & -7.6 & 22 \\
\hline 23 & $\sqrt{ }$ & & & & & & & & & & $\sqrt{ }$ & $\sqrt{ }$ & -6.0 & -1.0 & 30 \\
\hline 24 & $\sqrt{ }$ & $\sqrt{ }$ & & $\sqrt{ }$ & & & & & $\sqrt{ }$ & $\sqrt{ }$ & $\sqrt{ }$ & $\sqrt{ }$ & -4.3 & -3.6 & 32 \\
\hline 25 & $\sqrt{ }$ & $\sqrt{ }$ & $\sqrt{ }$ & & $\sqrt{ }$ & $\sqrt{ }$ & & & $\sqrt{ }$ & $\sqrt{ }$ & $\sqrt{ }$ & $\checkmark$ & -0.9 & -2.3 & 18 \\
\hline 26 & $\sqrt{ }$ & $\sqrt{ }$ & $\sqrt{ }$ & $\sqrt{ }$ & & $\sqrt{ }$ & & & & $\sqrt{ }$ & $\sqrt{ }$ & $\sqrt{ }$ & -4.8 & -6.4 & 34 \\
\hline 27 & $\sqrt{ }$ & $\sqrt{ }$ & & & & & & & & & $\sqrt{ }$ & $\sqrt{ }$ & -5.1 & -3.4 & 35 \\
\hline 28 & $\sqrt{ }$ & $\sqrt{ }$ & $\sqrt{ }$ & & $\sqrt{ }$ & & $\checkmark$ & & $\sqrt{ }$ & $\sqrt{ }$ & $\sqrt{ }$ & $\checkmark$ & -4.4 & -3.8 & 15 \\
\hline 29 & $\sqrt{ }$ & $\sqrt{ }$ & $\sqrt{ }$ & & & & & & $\sqrt{ }$ & $\sqrt{ }$ & & $\sqrt{ }$ & -6.9 & -4.8 & 25 \\
\hline 30 & $\sqrt{ }$ & & $\sqrt{ }$ & $\sqrt{ }$ & $\sqrt{ }$ & & & & $\sqrt{ }$ & $\sqrt{ }$ & $\sqrt{ }$ & $\sqrt{ }$ & -3.2 & -7.7 & 20 \\
\hline 31 & $\sqrt{ }$ & $\sqrt{ }$ & & & & & & & & $\sqrt{ }$ & $\sqrt{ }$ & $\sqrt{ }$ & -4.3 & -3.7 & 17 \\
\hline 32 & $\sqrt{ }$ & & & & & $\sqrt{ }$ & & & $\sqrt{ }$ & $\sqrt{ }$ & $\sqrt{ }$ & $\checkmark$ & -4.8 & -4.8 & 29 \\
\hline 33 & & $\sqrt{ }$ & $\sqrt{ }$ & & & & & & $\sqrt{ }$ & $\sqrt{ }$ & $\sqrt{ }$ & $\checkmark$ & -5.3 & -4.3 & 19 \\
\hline 34 & $\sqrt{ }$ & $\sqrt{ }$ & & $\sqrt{ }$ & $\sqrt{ }$ & $\sqrt{ }$ & & & $\sqrt{ }$ & $\sqrt{ }$ & $\sqrt{ }$ & $\sqrt{ }$ & -1.7 & -5.4 & 17 \\
\hline 35 & $\sqrt{ }$ & $\sqrt{ }$ & $\sqrt{ }$ & & & & & & & $\sqrt{ }$ & $\checkmark$ & $\checkmark$ & -6.5 & -2.8 & 20 \\
\hline 36 & $\sqrt{ }$ & & $\sqrt{ }$ & $\checkmark$ & & & & & $\sqrt{ }$ & $\sqrt{ }$ & & $\sqrt{ }$ & -5.9 & -4.3 & 17 \\
\hline 37 & $\sqrt{ }$ & & & $\sqrt{ }$ & & & & & $\sqrt{ }$ & $\sqrt{ }$ & $\sqrt{ }$ & $\sqrt{ }$ & -4.8 & -2.9 & 33 \\
\hline 38 & $\sqrt{ }$ & $\sqrt{ }$ & $\sqrt{ }$ & $\checkmark$ & $\sqrt{ }$ & & $\sqrt{ }$ & & $\sqrt{ }$ & $\sqrt{ }$ & $\sqrt{ }$ & $\sqrt{ }$ & -3.1 & -6.3 & 17 \\
\hline 39 & $\sqrt{ }$ & $\sqrt{ }$ & $\checkmark$ & $\checkmark$ & $\sqrt{ }$ & $\sqrt{ }$ & $\sqrt{ }$ & & $\sqrt{ }$ & $\sqrt{ }$ & $\sqrt{ }$ & $\checkmark$ & -3.5 & -4.5 & 16 \\
\hline 40 & $\sqrt{ }$ & & & $\sqrt{ }$ & & & & & & $\sqrt{ }$ & & $\checkmark$ & -5.1 & -4.1 & 18 \\
\hline 41 & $\sqrt{ }$ & $\sqrt{ }$ & & & & & & & $\sqrt{ }$ & & $\sqrt{ }$ & $\sqrt{ }$ & -6.1 & -3.7 & 22 \\
\hline 42 & $\sqrt{ }$ & $\sqrt{ }$ & & & $\checkmark$ & & $\sqrt{ }$ & & & $\sqrt{ }$ & $\sqrt{ }$ & $\checkmark$ & -2.9 & -5.9 & 6 \\
\hline 43 & $\sqrt{ }$ & $\sqrt{ }$ & & & $\checkmark$ & & $\checkmark$ & & $\sqrt{ }$ & & $\sqrt{ }$ & $\checkmark$ & -2.7 & -3.5 & 23 \\
\hline 44 & $\sqrt{ }$ & $\sqrt{ }$ & $\sqrt{ }$ & $\sqrt{ }$ & & & $\sqrt{ }$ & & $\sqrt{ }$ & V & $\sqrt{ }$ & $\sqrt{ }$ & -5.3 & -3.9 & 7 \\
\hline 45 & $\sqrt{ }$ & $\sqrt{ }$ & & & $\sqrt{ }$ & & & & & & $\sqrt{ }$ & $\sqrt{ }$ & -2.8 & -4.2 & 20 \\
\hline 46 & $\sqrt{ }$ & & & & $\sqrt{ }$ & $\sqrt{ }$ & & & & $\sqrt{ }$ & $\sqrt{ }$ & $\sqrt{ }$ & -3.0 & -0.4 & 40 \\
\hline 47 & $\sqrt{ }$ & & $\sqrt{ }$ & $\sqrt{ }$ & $\sqrt{ }$ & $\sqrt{ }$ & & & $\sqrt{ }$ & $\sqrt{ }$ & $\sqrt{ }$ & $\sqrt{ }$ & -2.6 & -2.8 & 6 \\
\hline 48 & $\sqrt{ }$ & $\sqrt{ }$ & & & & & $\sqrt{ }$ & & & & $\sqrt{ }$ & $\sqrt{ }$ & -4.4 & -1.0 & 13 \\
\hline 49 & $\sqrt{ }$ & $\sqrt{ }$ & $V$ & $\checkmark$ & & & & $\sqrt{ }$ & $\sqrt{ }$ & $\checkmark$ & $\sqrt{ }$ & $\checkmark$ & -5.5 & -5.6 & 15 \\
\hline 50 & & & $\sqrt{ }$ & & & & & & $\sqrt{ }$ & $\sqrt{ }$ & $\sqrt{ }$ & $\sqrt{ }$ & -6.5 & -4.9 & 15 \\
\hline
\end{tabular}


Table 48: Model's change in VTR prediction for worksites with Transit share between $0 \%$ to $5 \%$ and Vehicle Trip Rate between 80 to 90

\begin{tabular}{|c|c|c|c|c|c|c|c|c|c|c|c|c|c|c|c|}
\hline $\begin{array}{l}\text { Sr. } \\
\text { No. }\end{array}$ & $F \& A$ & ONS & СТВ & FI N & CWW & FLEX & TELE & PMT & NONF & GRH & RSMP & MRKT & $\begin{array}{c}\text { Trar } \\
\text { [C } \\
\text { VTR }\end{array}$ & $\begin{array}{l}\text { ssit Sha } \\
0-0.05) \\
-[90-8\end{array}$ & \\
\hline & & & & & & & & & & & & & Predicted & Actual & No.Ex \\
\hline 1 & $\sqrt{ }$ & $\sqrt{ }$ & $\sqrt{ }$ & v & & & & & $\sqrt{ }$ & v & $\sqrt{ }$ & $\sqrt{ }$ & -2.9 & -1.4 & 461 \\
\hline 2 & $\sqrt{ }$ & $\sqrt{ }$ & $\sqrt{ }$ & $\sqrt{ }$ & & $\sqrt{ }$ & & & $\sqrt{ }$ & $\sqrt{ }$ & $\sqrt{ }$ & $\sqrt{ }$ & -1.6 & -1.9 & 342 \\
\hline 3 & $\sqrt{ }$ & $\sqrt{ }$ & $\sqrt{ }$ & & & & & & $\sqrt{ }$ & $\sqrt{ }$ & $\sqrt{ }$ & $\sqrt{ }$ & -3.7 & -1.2 & 192 \\
\hline 4 & $\sqrt{ }$ & & $\sqrt{ }$ & $\sqrt{ }$ & & & & & $\sqrt{ }$ & $\sqrt{ }$ & V & $\sqrt{ }$ & -4.2 & -1.9 & 215 \\
\hline 5 & $\sqrt{ }$ & $\sqrt{ }$ & & & & & & & $\sqrt{ }$ & $\sqrt{ }$ & $\sqrt{ }$ & $\sqrt{ }$ & -2.1 & -0.9 & 174 \\
\hline 6 & & $\sqrt{ }$ & $\sqrt{ }$ & $\sqrt{ }$ & & & & & $\sqrt{ }$ & $\sqrt{ }$ & V & $\sqrt{ }$ & -3.2 & -1.7 & 115 \\
\hline 7 & $\sqrt{ }$ & $\sqrt{ }$ & & & & $\sqrt{ }$ & & & $\sqrt{ }$ & $\sqrt{ }$ & $\sqrt{ }$ & $\sqrt{ }$ & -1.9 & -2.3 & 126 \\
\hline 8 & $\sqrt{ }$ & $\sqrt{ }$ & $\sqrt{ }$ & $\sqrt{ }$ & $\sqrt{ }$ & & & & $\sqrt{ }$ & J & V & $\sqrt{ }$ & 0.0 & -1.6 & 142 \\
\hline 9 & $\sqrt{ }$ & & $\sqrt{ }$ & & & & & & $\sqrt{ }$ & $\sqrt{ }$ & $\sqrt{ }$ & $\sqrt{ }$ & -4.9 & -2.1 & 79 \\
\hline 10 & $\sqrt{ }$ & $\sqrt{ }$ & & & $\sqrt{ }$ & & & & $\sqrt{ }$ & $\sqrt{ }$ & $\sqrt{ }$ & $\sqrt{ }$ & -0.5 & -0.8 & 108 \\
\hline 11 & $\sqrt{ }$ & 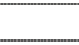 & $\sqrt{ }$ & $\checkmark$ & & & & & & $\sqrt{ }$ & $\sqrt{ }$ & $\sqrt{ }$ & -4.2 & -1.9 & 215 \\
\hline 12 & $\sqrt{ }$ & - & & & & & & & & $\sqrt{ }$ & V & $\sqrt{ }$ & -4.1 & -0.1 & 82 \\
\hline 13 & $\sqrt{ }$ & $\sqrt{ }$ & $\sqrt{ }$ & & & $\sqrt{ }$ & & & $\sqrt{ }$ & $\sqrt{ }$ & $\sqrt{ }$ & $\sqrt{ }$ & -2.9 & -1.2 & 93 \\
\hline 14 & $\sqrt{ }$ & $\sqrt{ }$ & $\sqrt{ }$ & $\sqrt{ }$ & & & & & & $\sqrt{ }$ & $\sqrt{ }$ & $\sqrt{ }$ & -2.6 & -2.1 & 77 \\
\hline 15 & $\sqrt{ }$ & & & & & & & & $\sqrt{ }$ & $\sqrt{ }$ & $\sqrt{ }$ & $\sqrt{ }$ & -3.7 & -2.3 & 69 \\
\hline 16 & $\sqrt{ }$ & $\sqrt{ }$ & $\sqrt{ }$ & $\checkmark$ & $\sqrt{ }$ & $\sqrt{ }$ & & & $\sqrt{ }$ & $\sqrt{ }$ & $\checkmark$ & $\checkmark$ & 0.6 & -1.8 & 103 \\
\hline 17 & $\sqrt{ }$ & $\sqrt{ }$ & V & $\checkmark$ & & & & & $\sqrt{ }$ & $\checkmark$ & & V & -3.3 & -0.9 & 89 \\
\hline 18 & $\sqrt{ }$ & $\sqrt{ }$ & $\checkmark$ & 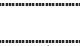 & $\sqrt{ }$ & & & & $\sqrt{ }$ & $\sqrt{ }$ & $\checkmark$ & $\sqrt{ }$ & -0.9 & -2.6 & 75 \\
\hline 19 & $\sqrt{ }$ & & $\sqrt{ }$ & $\sqrt{ }$ & & $\sqrt{ }$ & & & $\sqrt{ }$ & $\sqrt{ }$ & $\sqrt{ }$ & $\sqrt{ }$ & -3.4 & -3.3 & 64 \\
\hline 20 & & & $\sqrt{ }$ & $\sqrt{ }$ & & & & & $\sqrt{ }$ & $\sqrt{ }$ & $\sqrt{ }$ & $\sqrt{ }$ & -3.6 & -1.7 & 49 \\
\hline 21 & $\sqrt{ }$ & $\sqrt{ }$ & & & $\sqrt{ }$ & & $\sqrt{ }$ & & $\sqrt{ }$ & $\sqrt{ }$ & $\sqrt{ }$ & $\checkmark$ & 0.2 & -2.3 & 60 \\
\hline 22 & $\sqrt{ }$ & & $\sqrt{ }$ & & & $\sqrt{ }$ & & & $\sqrt{ }$ & $\sqrt{ }$ & $\sqrt{ }$ & $\sqrt{ }$ & -4.4 & -2.3 & 47 \\
\hline 23 & $\sqrt{ }$ & & & & & & & & & & $\sqrt{ }$ & $\sqrt{ }$ & -3.1 & 1.3 & 45 \\
\hline 24 & $\sqrt{ }$ & $\sqrt{ }$ & & $\sqrt{ }$ & & & & & $\sqrt{ }$ & $\sqrt{ }$ & $\sqrt{ }$ & $\sqrt{ }$ & -1.4 & -0.5 & 59 \\
\hline 25 & $\sqrt{ }$ & $\sqrt{ }$ & $\sqrt{ }$ & & $\sqrt{ }$ & $\sqrt{ }$ & & & $\sqrt{ }$ & $\sqrt{ }$ & $\sqrt{ }$ & $\sqrt{ }$ & -0.2 & -0.4 & 52 \\
\hline 26 & $\sqrt{ }$ & $\sqrt{ }$ & $\sqrt{ }$ & $\sqrt{ }$ & & $\sqrt{ }$ & & & & $\sqrt{ }$ & $\sqrt{ }$ & $\sqrt{ }$ & -1.3 & -3.0 & 47 \\
\hline 27 & $\sqrt{ }$ & $\sqrt{ }$ & & & & & & & & & V & $\sqrt{ }$ & -2.7 & -1.4 & 21 \\
\hline 28 & $\sqrt{ }$ & $\sqrt{ }$ & $\checkmark$ & & $\sqrt{ }$ & & $\sqrt{ }$ & & $\sqrt{ }$ & $\sqrt{ }$ & $\sqrt{ }$ & $\sqrt{ }$ & -0.6 & -1.2 & 51 \\
\hline 29 & $\sqrt{ }$ & $\sqrt{ }$ & $\sqrt{ }$ & & & & & & $\sqrt{ }$ & $\sqrt{ }$ & & $\sqrt{ }$ & -3.7 & -2.5 & 32 \\
\hline 30 & $\sqrt{ }$ & & $\sqrt{ }$ & $\sqrt{ }$ & $\sqrt{ }$ & & & & $\sqrt{ }$ & $\sqrt{ }$ & $\sqrt{ }$ & $\sqrt{ }$ & -1.6 & -1.6 & 47 \\
\hline 31 & $\sqrt{ }$ & $\sqrt{ }$ & & & & & & & & $\sqrt{ }$ & $\sqrt{ }$ & $\sqrt{ }$ & -2.0 & -3.0 & 22 \\
\hline 32 & $\sqrt{ }$ & & & & & $\sqrt{ }$ & & & $\sqrt{ }$ & $\checkmark$ & $\sqrt{ }$ & V & -3.3 & -2.4 & 32 \\
\hline 33 & & $\sqrt{ }$ & $\checkmark$ & & & & & & $\sqrt{ }$ & $\sqrt{ }$ & $\checkmark$ & $\sqrt{ }$ & -4.1 & -3.2 & 40 \\
\hline 34 & $\sqrt{ }$ & $\sqrt{ }$ & & $\sqrt{ }$ & $\sqrt{ }$ & $\sqrt{ }$ & & & $\sqrt{ }$ & $\sqrt{ }$ & V & $\sqrt{ }$ & 0.3 & 0.2 & 60 \\
\hline 35 & $\sqrt{ }$ & $\sqrt{ }$ & $\sqrt{ }$ & & & & & & & $\sqrt{ }$ & $\sqrt{ }$ & $\sqrt{ }$ & -2.9 & -1.7 & 35 \\
\hline 36 & $\sqrt{ }$ & & $\sqrt{ }$ & $\checkmark$ & & & & & $\sqrt{ }$ & $\sqrt{ }$ & & $\sqrt{ }$ & -3.5 & -2.7 & 52 \\
\hline 37 & $\sqrt{ }$ & & & $\sqrt{ }$ & & & & & $\sqrt{ }$ & $\sqrt{ }$ & $\sqrt{ }$ & $\sqrt{ }$ & -2.9 & -2.1 & 42 \\
\hline 38 & $\sqrt{ }$ & $\sqrt{ }$ & $\sqrt{ }$ & $\sqrt{ }$ & $\sqrt{ }$ & & $\sqrt{ }$ & & $\sqrt{ }$ & $\sqrt{ }$ & $\sqrt{ }$ & $\sqrt{ }$ & 0.1 & -0.9 & 46 \\
\hline 39 & $\sqrt{ }$ & $\sqrt{ }$ & $\sqrt{ }$ & $\checkmark$ & v & $\sqrt{ }$ & $\sqrt{ }$ & & $\sqrt{ }$ & $\checkmark$ & $\sqrt{ }$ & $\checkmark$ & -1.0 & -1.8 & 41 \\
\hline 40 & $\sqrt{ }$ & & & $\sqrt{ }$ & & & & & & $\sqrt{ }$ & & $\sqrt{ }$ & -2.1 & -0.7 & 57 \\
\hline 41 & $\sqrt{ }$ & $\sqrt{ }$ & & & & & & & $\sqrt{ }$ & & $\sqrt{ }$ & $\checkmark$ & -2.4 & -2.2 & 19 \\
\hline 42 & $\sqrt{ }$ & $\sqrt{ }$ & & & $\sqrt{ }$ & & $\sqrt{ }$ & & & $\checkmark$ & $\checkmark$ & $\checkmark$ & -0.5 & -1.1 & 44 \\
\hline 43 & $\sqrt{ }$ & $\sqrt{ }$ & & & $\sqrt{ }$ & & $\sqrt{ }$ & & $\sqrt{ }$ & & $\checkmark$ & $\checkmark$ & 0.7 & -1.5 & 36 \\
\hline 44 & $\sqrt{ }$ & $\sqrt{ }$ & $\sqrt{ }$ & $\sqrt{ }$ & & & $\sqrt{ }$ & & $\sqrt{ }$ & $\sqrt{ }$ & V & $\sqrt{ }$ & -2.1 & -0.4 & 45 \\
\hline 45 & $\sqrt{ }$ & $\sqrt{ }$ & & & $\sqrt{ }$ & & & & & & $\sqrt{ }$ & $\sqrt{ }$ & 0.3 & -0.3 & 30 \\
\hline 46 & $\sqrt{ }$ & & & & $\sqrt{ }$ & $\sqrt{ }$ & & & & $\sqrt{ }$ & $\sqrt{ }$ & $\sqrt{ }$ & -1.5 & 0.8 & 19 \\
\hline 47 & $\sqrt{ }$ & & $\sqrt{ }$ & $\sqrt{ }$ & $\sqrt{ }$ & $\sqrt{ }$ & & & $\sqrt{ }$ & $\sqrt{ }$ & $\sqrt{ }$ & $\sqrt{ }$ & -1.6 & -3.9 & 34 \\
\hline 48 & $\sqrt{ }$ & $\sqrt{ }$ & & & & & $\checkmark$ & & & & $\checkmark$ & $\checkmark$ & -3.1 & 0.8 & 33 \\
\hline 49 & $\sqrt{ }$ & $\sqrt{ }$ & $\sqrt{ }$ & $\checkmark$ & & & & $\sqrt{ }$ & $\sqrt{ }$ & $\checkmark$ & $\checkmark$ & $\checkmark$ & -2.4 & -3.5 & 22 \\
\hline 50 & & & $\sqrt{ }$ & & & & & & $\sqrt{ }$ & $\sqrt{ }$ & V & $\sqrt{ }$ & -3.9 & -0.1 & 16 \\
\hline
\end{tabular}


Table 49: Model's change in VTR prediction for worksites with Transit share between $0 \%$ to $5 \%$ and Vehicle Trip Rate between 70 to 80

\begin{tabular}{|c|c|c|c|c|c|c|c|c|c|c|c|c|c|c|c|}
\hline $\begin{array}{l}\text { Sr. } \\
\text { No. }\end{array}$ & $F \& A$ & ONS & СТВ & FI N & CWW & FLEX & TELE & PMT & NONF & GRH & RSMP & MRKT & $\begin{array}{r}\text { Tran } \\
\text { [0 } \\
\text { VTR }\end{array}$ & $\begin{array}{l}\text { ssit Sha } \\
0-0.05) \\
-[80-7\end{array}$ & \\
\hline & & & & & & & & & & & & & Predicted & Actual & No.Ex \\
\hline 1 & $\sqrt{ }$ & $\sqrt{ }$ & $\sqrt{ }$ & $\sqrt{ }$ & & & & & $\sqrt{ }$ & $\sqrt{ }$ & $\sqrt{ }$ & $\sqrt{ }$ & -0.6 & 1.0 & 147 \\
\hline 2 & $\sqrt{ }$ & $\sqrt{ }$ & $\sqrt{ }$ & $\sqrt{ }$ & & $\sqrt{ }$ & & & $\sqrt{ }$ & $\sqrt{ }$ & $\sqrt{ }$ & $\sqrt{ }$ & 0.4 & 1.6 & 88 \\
\hline 3 & $\sqrt{ }$ & $\sqrt{ }$ & $\sqrt{ }$ & & & & & & $\sqrt{ }$ & $\sqrt{ }$ & $\sqrt{ }$ & $\sqrt{ }$ & -1.0 & 0.5 & 93 \\
\hline 4 & $\sqrt{ }$ & & $\sqrt{ }$ & $\sqrt{ }$ & & & & & $\sqrt{ }$ & $\sqrt{ }$ & $\sqrt{ }$ & $\sqrt{ }$ & -1.6 & 1.2 & 90 \\
\hline 5 & $\sqrt{ }$ & $\sqrt{ }$ & & & & & & & $\sqrt{ }$ & $\sqrt{ }$ & $\sqrt{ }$ & $\sqrt{ }$ & -0.1 & -0.1 & 71 \\
\hline 6 & & $\sqrt{ }$ & $\sqrt{ }$ & $\sqrt{ }$ & & & & & $\sqrt{ }$ & $\sqrt{ }$ & $\sqrt{ }$ & $\sqrt{ }$ & -0.4 & -0.2 & 29 \\
\hline 7 & $\sqrt{ }$ & $\sqrt{ }$ & & & & $\sqrt{ }$ & & & $\sqrt{ }$ & $\sqrt{ }$ & $\sqrt{ }$ & $\sqrt{ }$ & 0.8 & 1.2 & 34 \\
\hline 8 & $\sqrt{ }$ & $\sqrt{ }$ & $\sqrt{ }$ & $\sqrt{ }$ & $\sqrt{ }$ & & & & $\sqrt{ }$ & $\sqrt{ }$ & $\sqrt{ }$ & $\sqrt{ }$ & 1.6 & 0.6 & 78 \\
\hline 9 & $\sqrt{ }$ & & $\sqrt{ }$ & & & & & & $\sqrt{ }$ & $\sqrt{ }$ & $\sqrt{ }$ & $\sqrt{ }$ & -2.1 & 1.2 & 44 \\
\hline 10 & $\sqrt{ }$ & $\sqrt{ }$ & & & $\sqrt{ }$ & & & & $\sqrt{ }$ & $\sqrt{ }$ & $\sqrt{ }$ & $\sqrt{ }$ & 1.7 & -0.3 & 49 \\
\hline 11 & $\sqrt{ }$ & & $\sqrt{ }$ & $\checkmark$ & & & & & & $\sqrt{ }$ & $\sqrt{ }$ & $\sqrt{ }$ & -1.6 & 1.2 & 90 \\
\hline 12 & $\sqrt{ }$ & 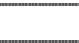 & & & & & & & & $\sqrt{ }$ & $\sqrt{ }$ & $\sqrt{ }$ & -1.0 & 0.5 & 11 \\
\hline 13 & $\sqrt{ }$ & $\sqrt{ }$ & $\sqrt{ }$ & & & $\sqrt{ }$ & & & $\sqrt{ }$ & $\sqrt{ }$ & $\sqrt{ }$ & $\sqrt{ }$ & -0.6 & 1.2 & 28 \\
\hline 14 & $\sqrt{ }$ & $\sqrt{ }$ & $\sqrt{ }$ & $\checkmark$ & & & & & & $\sqrt{ }$ & $\checkmark$ & $\sqrt{ }$ & -0.1 & -0.5 & 26 \\
\hline 15 & $\sqrt{ }$ & & & & & & & & $\sqrt{ }$ & $\sqrt{ }$ & $\sqrt{ }$ & $\sqrt{ }$ & -1.1 & -0.9 & 49 \\
\hline 16 & $\sqrt{ }$ & $\sqrt{ }$ & $\sqrt{ }$ & $\sqrt{ }$ & $\sqrt{ }$ & $\sqrt{ }$ & & & $\sqrt{ }$ & $\sqrt{ }$ & $\sqrt{ }$ & $\sqrt{ }$ & 2.5 & 0.4 & 41 \\
\hline 17 & $\sqrt{ }$ & $\sqrt{ }$ & $\sqrt{ }$ & $\sqrt{ }$ & & & & & $\sqrt{ }$ & $\sqrt{ }$ & & $\checkmark$ & -2.1 & -3.6 & 23 \\
\hline 18 & $\sqrt{ }$ & $\sqrt{ }$ & $\checkmark$ & & $\checkmark$ & & & & $\sqrt{ }$ & $\checkmark$ & $\sqrt{ }$ & $\checkmark$ & 1.2 & 2.2 & 44 \\
\hline 19 & $\sqrt{ }$ & & $\sqrt{ }$ & $\sqrt{ }$ & & $\sqrt{ }$ & & & $\sqrt{ }$ & $\sqrt{ }$ & $\sqrt{ }$ & $\sqrt{ }$ & -1.6 & 0.9 & 15 \\
\hline 20 & & & $\sqrt{ }$ & $\sqrt{ }$ & & & & & $\sqrt{ }$ & $\sqrt{ }$ & $\sqrt{ }$ & $\sqrt{ }$ & -2.1 & 1.8 & 16 \\
\hline 21 & $\sqrt{ }$ & $\sqrt{ }$ & & & V & & $\sqrt{ }$ & & $\sqrt{ }$ & $\sqrt{ }$ & $\sqrt{ }$ & $\checkmark$ & 2.8 & -1.0 & 19 \\
\hline 22 & $\sqrt{ }$ & & $\sqrt{ }$ & & & $\sqrt{ }$ & & & $\sqrt{ }$ & $\sqrt{ }$ & $\sqrt{ }$ & $\sqrt{ }$ & -3.5 & -0.8 & 13 \\
\hline 23 & $\sqrt{ }$ & & & & & & & & & & $\sqrt{ }$ & $\sqrt{ }$ & -1.4 & -2.4 & 6 \\
\hline 24 & $\sqrt{ }$ & $\sqrt{ }$ & & $\sqrt{ }$ & & & & & $\sqrt{ }$ & $\sqrt{ }$ & $\sqrt{ }$ & $\sqrt{ }$ & -0.3 & 0.8 & 18 \\
\hline 25 & $\sqrt{ }$ & $\sqrt{ }$ & $\sqrt{ }$ & & $\sqrt{ }$ & $\sqrt{ }$ & & & $\sqrt{ }$ & $\sqrt{ }$ & $\sqrt{ }$ & $\checkmark$ & 2.0 & 0.9 & 25 \\
\hline 26 & $\sqrt{ }$ & $\sqrt{ }$ & $\sqrt{ }$ & $\sqrt{ }$ & & $\sqrt{ }$ & & & & $\sqrt{ }$ & $\sqrt{ }$ & $\sqrt{ }$ & 1.0 & 0.2 & 11 \\
\hline 27 & $\sqrt{ }$ & $\sqrt{ }$ & & & & & & & & & $\sqrt{ }$ & $\sqrt{ }$ & -0.4 & 5.0 & 1 \\
\hline 28 & $\sqrt{ }$ & $\sqrt{ }$ & $\checkmark$ & & $\checkmark$ & & $\checkmark$ & & $\sqrt{ }$ & $\sqrt{ }$ & $\sqrt{ }$ & $\checkmark$ & 1.8 & 1.3 & 29 \\
\hline 29 & $\sqrt{ }$ & $\sqrt{ }$ & $\sqrt{ }$ & & & & & & $\sqrt{ }$ & $\sqrt{ }$ & & $\sqrt{ }$ & -1.2 & 3.6 & 18 \\
\hline 30 & $\sqrt{ }$ & & $\sqrt{ }$ & $\sqrt{ }$ & $\sqrt{ }$ & & & & $\sqrt{ }$ & $\sqrt{ }$ & $\sqrt{ }$ & $\sqrt{ }$ & 0.9 & -1.3 & 19 \\
\hline 31 & $\sqrt{ }$ & $\sqrt{ }$ & & & & & & & & $\sqrt{ }$ & $\sqrt{ }$ & $\sqrt{ }$ & 0.0 & -2.2 & 9 \\
\hline 32 & $\sqrt{ }$ & & & & & $\sqrt{ }$ & & & $\sqrt{ }$ & $\sqrt{ }$ & $\sqrt{ }$ & $\checkmark$ & -1.2 & -0.7 & 17 \\
\hline 33 & & $\sqrt{ }$ & $\sqrt{ }$ & & & & & & $\sqrt{ }$ & $\sqrt{ }$ & $\sqrt{ }$ & $\checkmark$ & -0.9 & -2.9 & 10 \\
\hline 34 & $\sqrt{ }$ & $\sqrt{ }$ & & $\sqrt{ }$ & $\sqrt{ }$ & $\sqrt{ }$ & & & $\sqrt{ }$ & $\sqrt{ }$ & $\sqrt{ }$ & $\sqrt{ }$ & 2.7 & 0.7 & 13 \\
\hline 35 & $\sqrt{ }$ & $\sqrt{ }$ & $\sqrt{ }$ & & & & & & & $\sqrt{ }$ & $\sqrt{ }$ & $\checkmark$ & 0.1 & 0.3 & 10 \\
\hline 36 & $\sqrt{ }$ & & $\sqrt{ }$ & $\checkmark$ & & & & & $\sqrt{ }$ & $\sqrt{ }$ & & $\sqrt{ }$ & -1.4 & -1.5 & 15 \\
\hline 37 & $\sqrt{ }$ & & & $\sqrt{ }$ & & & & & $\sqrt{ }$ & $\sqrt{ }$ & $\sqrt{ }$ & $\sqrt{ }$ & -0.5 & 1.1 & 18 \\
\hline 38 & $\sqrt{ }$ & $\sqrt{ }$ & $\sqrt{ }$ & $\sqrt{ }$ & $\sqrt{ }$ & & $\sqrt{ }$ & & $\sqrt{ }$ & $\sqrt{ }$ & $\sqrt{ }$ & $\sqrt{ }$ & 2.4 & 1.3 & 19 \\
\hline 39 & $\sqrt{ }$ & $\sqrt{ }$ & $\checkmark$ & $\checkmark$ & $\sqrt{ }$ & $\sqrt{ }$ & $\sqrt{ }$ & & $\sqrt{ }$ & $\sqrt{ }$ & $\sqrt{ }$ & $\checkmark$ & 2.1 & 1.4 & 22 \\
\hline 40 & $\sqrt{ }$ & & & $\sqrt{ }$ & & & & & & $\sqrt{ }$ & & $\checkmark$ & -0.9 & -1.8 & 10 \\
\hline 41 & $\sqrt{ }$ & $\sqrt{ }$ & & & & & & & $\sqrt{ }$ & & $\sqrt{ }$ & $\sqrt{ }$ & 1.0 & 5.8 & 8 \\
\hline 42 & $\sqrt{ }$ & $\sqrt{ }$ & & & $\checkmark$ & & $\sqrt{ }$ & & & $\sqrt{ }$ & $\sqrt{ }$ & $\checkmark$ & 2.5 & 0.4 & 17 \\
\hline 43 & $\sqrt{ }$ & $\sqrt{ }$ & & & $\checkmark$ & & $\checkmark$ & & $\sqrt{ }$ & & $\sqrt{ }$ & $\checkmark$ & 2.9 & -2.4 & 8 \\
\hline 44 & $\sqrt{ }$ & $\sqrt{ }$ & $\sqrt{ }$ & $\sqrt{ }$ & & & $\sqrt{ }$ & & $\sqrt{ }$ & V & $\sqrt{ }$ & $\sqrt{ }$ & -0.6 & 0.0 & 14 \\
\hline 45 & $\sqrt{ }$ & $\sqrt{ }$ & & & $\sqrt{ }$ & & & & & & $\sqrt{ }$ & $\sqrt{ }$ & 0.8 & -2.8 & 13 \\
\hline 46 & $\sqrt{ }$ & & & & $\sqrt{ }$ & $\sqrt{ }$ & & & & $\sqrt{ }$ & $\sqrt{ }$ & $\sqrt{ }$ & 1.3 & 1.4 & 12 \\
\hline 47 & $\sqrt{ }$ & & $\sqrt{ }$ & $\sqrt{ }$ & $\sqrt{ }$ & $\sqrt{ }$ & & & $\sqrt{ }$ & $\sqrt{ }$ & $\sqrt{ }$ & $\sqrt{ }$ & 0.7 & 0.7 & 23 \\
\hline 48 & $\sqrt{ }$ & $\sqrt{ }$ & & & & & $\sqrt{ }$ & & & & $\sqrt{ }$ & $\checkmark$ & -0.9 & 3.4 & 4 \\
\hline 49 & $\sqrt{ }$ & $\sqrt{ }$ & $\sqrt{ }$ & $\checkmark$ & & & & $\sqrt{ }$ & $\sqrt{ }$ & $\checkmark$ & $\sqrt{ }$ & $\sqrt{ }$ & 0.7 & -2.4 & 8 \\
\hline 50 & & & $\sqrt{ }$ & & & & & & $\sqrt{ }$ & $\sqrt{ }$ & $\sqrt{ }$ & $\sqrt{ }$ & -1.9 & 3.2 & 5 \\
\hline
\end{tabular}


Table 50: Model's change in VTR prediction for worksites with Transit share between $0 \%$ to $5 \%$ and Vehicle Trip Rate between 60 to 70

\begin{tabular}{|c|c|c|c|c|c|c|c|c|c|c|c|c|c|c|c|}
\hline $\begin{array}{l}\text { Sr. } \\
\text { No. }\end{array}$ & F\&A & ONS & СТВ & FI N & CWW & FLEX & TELE & PMT & NONF & GRH & RSMP & MRKT & $\begin{array}{r}\text { Tran } \\
{[0} \\
\text { VTR }\end{array}$ & $\begin{array}{l}\text { nsit Sha } \\
0-0.05) \\
-[70-60\end{array}$ & \\
\hline & & & & & & & & & & & & & Predicted & Actual & No.Ex \\
\hline 1 & $\sqrt{ }$ & $\sqrt{ }$ & $\sqrt{ }$ & $\sqrt{ }$ & & & & & $\sqrt{ }$ & $\sqrt{ }$ & $\sqrt{ }$ & $\sqrt{ }$ & 0.6 & 2.5 & 27 \\
\hline 2 & $\sqrt{ }$ & $\sqrt{ }$ & $\sqrt{ }$ & $\sqrt{ }$ & & $\sqrt{ }$ & & & $\sqrt{ }$ & $\sqrt{ }$ & $\sqrt{ }$ & $\sqrt{ }$ & 2.5 & 2.0 & 16 \\
\hline 3 & $\sqrt{ }$ & $\sqrt{ }$ & $\sqrt{ }$ & & & & & & $\sqrt{ }$ & $\sqrt{ }$ & $\sqrt{ }$ & $\sqrt{ }$ & -0.1 & 2.4 & 23 \\
\hline 4 & $\sqrt{ }$ & & $\sqrt{ }$ & $\sqrt{ }$ & & & & & $\sqrt{ }$ & $\sqrt{ }$ & $\sqrt{ }$ & $\sqrt{ }$ & 1.3 & 0.4 & 15 \\
\hline 5 & $\sqrt{ }$ & $\sqrt{ }$ & & & & & & & $\sqrt{ }$ & $\sqrt{ }$ & $\sqrt{ }$ & $\sqrt{ }$ & 1.5 & 3.9 & 28 \\
\hline 6 & & $\sqrt{ }$ & $\sqrt{ }$ & $\sqrt{ }$ & & & & & $\sqrt{ }$ & $\sqrt{ }$ & $\sqrt{ }$ & $\sqrt{ }$ & -3.0 & -4.8 & 2 \\
\hline 7 & $\sqrt{ }$ & $\sqrt{ }$ & & & & $\sqrt{ }$ & & & $\sqrt{ }$ & $\sqrt{ }$ & $\sqrt{ }$ & $\sqrt{ }$ & 2.7 & 5.2 & 7 \\
\hline 8 & $\sqrt{ }$ & $\sqrt{ }$ & $\sqrt{ }$ & $\sqrt{ }$ & $\sqrt{ }$ & & & & $\sqrt{ }$ & $\sqrt{ }$ & $\sqrt{ }$ & $\sqrt{ }$ & 4.5 & 5.1 & 19 \\
\hline 9 & $\sqrt{ }$ & & $\sqrt{ }$ & & & & & & $\sqrt{ }$ & $\sqrt{ }$ & $\sqrt{ }$ & $\sqrt{ }$ & 0.2 & 0.9 & 21 \\
\hline 10 & $\sqrt{ }$ & $\sqrt{ }$ & & & $\sqrt{ }$ & & & & $\sqrt{ }$ & $\sqrt{ }$ & $\sqrt{ }$ & $\sqrt{ }$ & 3.9 & 1.0 & 14 \\
\hline 11 & $\sqrt{ }$ & & $\sqrt{ }$ & $\sqrt{ }$ & & & & & & $\sqrt{ }$ & $\sqrt{ }$ & $\sqrt{ }$ & 1.3 & 0.4 & 15 \\
\hline 12 & $\sqrt{ }$ & - & & & & & & & & $\sqrt{ }$ & $\sqrt{ }$ & $\sqrt{ }$ & 1.5 & 0.8 & 15 \\
\hline 13 & $\sqrt{ }$ & $\sqrt{ }$ & $\sqrt{ }$ & & & $\sqrt{ }$ & & & $\sqrt{ }$ & $\sqrt{ }$ & $\sqrt{ }$ & $\sqrt{ }$ & 1.0 & 1.6 & 13 \\
\hline 14 & $\sqrt{ }$ & $\sqrt{ }$ & $\sqrt{ }$ & $\sqrt{ }$ & & & & & & $\sqrt{ }$ & $\checkmark$ & $\sqrt{ }$ & 1.5 & 1.0 & 9 \\
\hline 15 & $\sqrt{ }$ & & & & & & & & $\sqrt{ }$ & $\sqrt{ }$ & $\sqrt{ }$ & $\sqrt{ }$ & 1.1 & 0.7 & 19 \\
\hline 16 & $\sqrt{ }$ & $\sqrt{ }$ & $\sqrt{ }$ & $\sqrt{ }$ & $\sqrt{ }$ & $\sqrt{ }$ & & & $\sqrt{ }$ & $\sqrt{ }$ & $\sqrt{ }$ & $\sqrt{ }$ & 4.3 & 3.3 & 8 \\
\hline 17 & $\sqrt{ }$ & $\sqrt{ }$ & $\checkmark$ & $\sqrt{ }$ & & & & & $\sqrt{ }$ & $\sqrt{ }$ & & $\checkmark$ & -2.4 & 3.8 & 3 \\
\hline 18 & $\sqrt{ }$ & $\sqrt{ }$ & $\checkmark$ & ing & $\sqrt{ }$ & & & & $\sqrt{ }$ & $\sqrt{ }$ & $\sqrt{ }$ & $\checkmark$ & 3.4 & 0.8 & 20 \\
\hline 19 & $\sqrt{ }$ & & $\sqrt{ }$ & $\sqrt{ }$ & & $\sqrt{ }$ & & & $\sqrt{ }$ & $\sqrt{ }$ & $\sqrt{ }$ & $\sqrt{ }$ & 1.7 & 2.1 & 9 \\
\hline 20 & & & $\sqrt{ }$ & $\sqrt{ }$ & & & & & $\sqrt{ }$ & $\sqrt{ }$ & $\sqrt{ }$ & $\sqrt{ }$ & -1.0 & 3.8 & 6 \\
\hline 21 & $\sqrt{ }$ & $\sqrt{ }$ & & & $\sqrt{ }$ & & $\sqrt{ }$ & & $\sqrt{ }$ & $\sqrt{ }$ & $\sqrt{ }$ & $\checkmark$ & 5.0 & 5.0 & 9 \\
\hline 22 & $\sqrt{ }$ & & $\sqrt{ }$ & & & $\sqrt{ }$ & & & $\sqrt{ }$ & $\sqrt{ }$ & $\sqrt{ }$ & $\checkmark$ & -0.6 & 3.4 & 5 \\
\hline 23 & $\sqrt{ }$ & & & & & & & & & & $\checkmark$ & $\sqrt{ }$ & 2.3 & 0.5 & 10 \\
\hline 24 & $\sqrt{ }$ & $\sqrt{ }$ & & $\sqrt{ }$ & & & & & $\sqrt{ }$ & $\sqrt{ }$ & $\sqrt{ }$ & $\sqrt{ }$ & -0.4 & 0.5 & 3 \\
\hline 25 & $\sqrt{ }$ & $\sqrt{ }$ & $\sqrt{ }$ & & $\sqrt{ }$ & $\sqrt{ }$ & & & $\sqrt{ }$ & $\sqrt{ }$ & $\sqrt{ }$ & $\checkmark$ & 2.8 & 1.1 & 6 \\
\hline 26 & $\sqrt{ }$ & $\sqrt{ }$ & $\sqrt{ }$ & $\sqrt{ }$ & & $\sqrt{ }$ & & & & $\sqrt{ }$ & $\sqrt{ }$ & $\sqrt{ }$ & 0.9 & 1.8 & 5 \\
\hline 27 & $\sqrt{ }$ & $\sqrt{ }$ & & & & & & & & & $\sqrt{ }$ & $\sqrt{ }$ & 1.5 & 7.5 & 7 \\
\hline 28 & $\sqrt{ }$ & $\sqrt{ }$ & $\sqrt{ }$ & & $\sqrt{ }$ & & $\sqrt{ }$ & & $\sqrt{ }$ & $\sqrt{ }$ & $\sqrt{ }$ & $\checkmark$ & 3.1 & 4.0 & 5 \\
\hline 29 & $\sqrt{ }$ & $\sqrt{ }$ & $\sqrt{ }$ & & & & & & $\sqrt{ }$ & $\sqrt{ }$ & & $\sqrt{ }$ & -4.8 & 7.8 & 1 \\
\hline 30 & $\sqrt{ }$ & & $\sqrt{ }$ & $\sqrt{ }$ & $\sqrt{ }$ & & & & $\sqrt{ }$ & $\sqrt{ }$ & $\sqrt{ }$ & $\sqrt{ }$ & 3.7 & -0.6 & 8 \\
\hline 31 & $\sqrt{ }$ & $\sqrt{ }$ & & & & & & & & $\sqrt{ }$ & $\sqrt{ }$ & $\sqrt{ }$ & 1.2 & 2.0 & 18 \\
\hline 32 & $\sqrt{ }$ & & & & & $\sqrt{ }$ & & & $\sqrt{ }$ & $\sqrt{ }$ & $\sqrt{ }$ & $\checkmark$ & 0.5 & 8.5 & 3 \\
\hline 33 & & $\sqrt{ }$ & $\sqrt{ }$ & & & & & & $\sqrt{ }$ & $\sqrt{ }$ & $\sqrt{ }$ & $\checkmark$ & 0.5 & 2.8 & 4 \\
\hline 34 & $\sqrt{ }$ & $\sqrt{ }$ & & $\sqrt{ }$ & $\sqrt{ }$ & $\sqrt{ }$ & & & $\sqrt{ }$ & $\sqrt{ }$ & $\sqrt{ }$ & $\sqrt{ }$ & - & - & - \\
\hline 35 & $\sqrt{ }$ & $\sqrt{ }$ & $\sqrt{ }$ & & & & & & & $\sqrt{ }$ & $\checkmark$ & $\checkmark$ & 0.3 & 10.7 & 3 \\
\hline 36 & $\sqrt{ }$ & & $\sqrt{ }$ & $\sqrt{ }$ & & & & & $\sqrt{ }$ & $\sqrt{ }$ & & $\sqrt{ }$ & 0.1 & 4.1 & 6 \\
\hline 37 & $\sqrt{ }$ & & & $\sqrt{ }$ & & & & & $\sqrt{ }$ & $\sqrt{ }$ & $\sqrt{ }$ & $\sqrt{ }$ & 2.3 & 4.1 & 3 \\
\hline 38 & $\sqrt{ }$ & $\sqrt{ }$ & $\sqrt{ }$ & $\sqrt{ }$ & $\sqrt{ }$ & & $\sqrt{ }$ & & $\sqrt{ }$ & $\sqrt{ }$ & $\sqrt{ }$ & $\sqrt{ }$ & 4.9 & 4.6 & 4 \\
\hline 39 & $\sqrt{ }$ & $\sqrt{ }$ & $\checkmark$ & $\sqrt{ }$ & $\sqrt{ }$ & $\sqrt{ }$ & $\sqrt{ }$ & & $\sqrt{ }$ & $\sqrt{ }$ & $\sqrt{ }$ & $\checkmark$ & 1.0 & 1.7 & 5 \\
\hline 40 & $\sqrt{ }$ & & & $\sqrt{ }$ & & & & & & $\sqrt{ }$ & & $\checkmark$ & 0.9 & 13.6 & 1 \\
\hline 41 & $\sqrt{ }$ & $\sqrt{ }$ & & & & & & & $\sqrt{ }$ & & $\sqrt{ }$ & $\sqrt{ }$ & 3.2 & 4.5 & 10 \\
\hline 42 & $\sqrt{ }$ & $\sqrt{ }$ & & & $\checkmark$ & & $\sqrt{ }$ & & & $\sqrt{ }$ & $\sqrt{ }$ & $\checkmark$ & 4.1 & 1.8 & 1 \\
\hline 43 & $\sqrt{ }$ & $\sqrt{ }$ & & & $\sqrt{ }$ & & $\checkmark$ & & $\sqrt{ }$ & & $\sqrt{ }$ & $\checkmark$ & - & - & - \\
\hline 44 & $\sqrt{ }$ & $\sqrt{ }$ & $\sqrt{ }$ & $\sqrt{ }$ & & & $\sqrt{ }$ & & $\sqrt{ }$ & $\sqrt{ }$ & $\sqrt{ }$ & 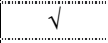 & 1.5 & -0.8 & 2 \\
\hline 45 & $\sqrt{ }$ & $\sqrt{ }$ & & & $\sqrt{ }$ & & & & & & $\sqrt{ }$ & $\sqrt{ }$ & 6.4 & 10.9 & 1 \\
\hline 46 & $\sqrt{ }$ & & & & $\sqrt{ }$ & $\sqrt{ }$ & & & & $\sqrt{ }$ & $\sqrt{ }$ & $\sqrt{ }$ & - & - & - \\
\hline 47 & $\sqrt{ }$ & & $\sqrt{ }$ & $\sqrt{ }$ & $\sqrt{ }$ & $\sqrt{ }$ & & & $\sqrt{ }$ & $\sqrt{ }$ & $\sqrt{ }$ & $\sqrt{ }$ & 2.6 & 4.7 & 7 \\
\hline 48 & $\sqrt{ }$ & $\sqrt{ }$ & & & & & $\sqrt{ }$ & & & & $\sqrt{ }$ & $\checkmark$ & -2.4 & 13.1 & 1 \\
\hline 49 & $\sqrt{ }$ & $\sqrt{ }$ & $\sqrt{ }$ & $\sqrt{ }$ & & & & $\sqrt{ }$ & $\sqrt{ }$ & $\sqrt{ }$ & $\sqrt{ }$ & $\checkmark$ & 2.0 & 3.3 & 5 \\
\hline 50 & & & $\sqrt{ }$ & & & & & & $\sqrt{ }$ & $\sqrt{ }$ & $\sqrt{ }$ & $\sqrt{ }$ & 1.0 & 5.2 & 3 \\
\hline
\end{tabular}


Table 51: Model's change in VTR prediction for worksites with Transit share between $0 \%$ to $5 \%$ and Vehicle Trip Rate between 50 to 60

\begin{tabular}{|c|c|c|c|c|c|c|c|c|c|c|c|c|c|c|c|}
\hline $\begin{array}{l}\text { Sr. } \\
\text { No. }\end{array}$ & $F \& A$ & ONS & СТВ & FI N & CWW & FLEX & TELE & PMT & NONF & GRH & RSMP & MRKT & $\begin{array}{r}\text { Tran } \\
\text { [0 } \\
\text { VTR } \\
\end{array}$ & $\begin{array}{l}\text { nsit Sha } \\
0-0.05) \\
-[60-50\end{array}$ & \\
\hline & & & & & & & & & & & & & Predicted & Actual & No.Ex \\
\hline 1 & $\sqrt{ }$ & $\sqrt{ }$ & $\sqrt{ }$ & $\sqrt{ }$ & & & & & $\sqrt{ }$ & $\sqrt{ }$ & $\sqrt{ }$ & $\sqrt{ }$ & 2.2 & 5.5 & 5 \\
\hline 2 & $\sqrt{ }$ & $\sqrt{ }$ & $\sqrt{ }$ & $\sqrt{ }$ & & $\sqrt{ }$ & & & $\sqrt{ }$ & $\sqrt{ }$ & $\sqrt{ }$ & $\sqrt{ }$ & -1.9 & 3.7 & 1 \\
\hline 3 & $\sqrt{ }$ & $\sqrt{ }$ & $\sqrt{ }$ & & & & & & $\sqrt{ }$ & $\sqrt{ }$ & $\sqrt{ }$ & $\sqrt{ }$ & 3.1 & 4.6 & 5 \\
\hline 4 & $\sqrt{ }$ & & $\sqrt{ }$ & $\sqrt{ }$ & & & & & $\sqrt{ }$ & $\sqrt{ }$ & $\sqrt{ }$ & $\sqrt{ }$ & - & - & - \\
\hline 5 & $\sqrt{ }$ & $\sqrt{ }$ & & & & & & & $\sqrt{ }$ & $\sqrt{ }$ & $\sqrt{ }$ & $\sqrt{ }$ & 3.5 & 21.9 & 3 \\
\hline 6 & & $\sqrt{ }$ & $\sqrt{ }$ & $\sqrt{ }$ & & & & & $\sqrt{ }$ & $\sqrt{ }$ & $\sqrt{ }$ & $\sqrt{ }$ & - & - & - \\
\hline 7 & $\sqrt{ }$ & $\sqrt{ }$ & & & & $\sqrt{ }$ & & & $\sqrt{ }$ & $\sqrt{ }$ & $\sqrt{ }$ & $\sqrt{ }$ & 2.1 & 5.7 & 1 \\
\hline 8 & $\sqrt{ }$ & $\sqrt{ }$ & $\sqrt{ }$ & $\sqrt{ }$ & $\sqrt{ }$ & & & & $\sqrt{ }$ & $\sqrt{ }$ & $\sqrt{ }$ & $\sqrt{ }$ & 6.6 & -0.2 & 2 \\
\hline 9 & $\sqrt{ }$ & & $\sqrt{ }$ & & & & & & $\sqrt{ }$ & $\sqrt{ }$ & $\sqrt{ }$ & $\sqrt{ }$ & 1.6 & 5.2 & 2 \\
\hline 10 & $\sqrt{ }$ & $\sqrt{ }$ & & & $\sqrt{ }$ & & & & $\sqrt{ }$ & $\sqrt{ }$ & $\sqrt{ }$ & $\sqrt{ }$ & 4.8 & 1.4 & 1 \\
\hline 11 & $\sqrt{ }$ & & $\sqrt{ }$ & $\sqrt{ }$ & & & & & & $\sqrt{ }$ & $\sqrt{ }$ & $\sqrt{ }$ & - & - & - \\
\hline 12 & $\sqrt{ }$ & & & & & & & & & $\sqrt{ }$ & $\sqrt{ }$ & $\sqrt{ }$ & 1.1 & -1.6 & 2 \\
\hline 13 & $\sqrt{ }$ & $\sqrt{ }$ & $\sqrt{ }$ & & & $\sqrt{ }$ & & & $\sqrt{ }$ & $\sqrt{ }$ & $\sqrt{ }$ & $\checkmark$ & 7.6 & 31.9 & 1 \\
\hline 14 & $\sqrt{ }$ & $\sqrt{ }$ & $\sqrt{ }$ & $\sqrt{ }$ & & & & & & $\sqrt{ }$ & $\sqrt{ }$ & $\checkmark$ & - & - & - \\
\hline 15 & $\sqrt{ }$ & & & & & & & & $\sqrt{ }$ & $\sqrt{ }$ & $\sqrt{ }$ & $\sqrt{ }$ & 3.1 & 1.7 & 1 \\
\hline 16 & $\sqrt{ }$ & $\sqrt{ }$ & $\sqrt{ }$ & $\sqrt{ }$ & $\sqrt{ }$ & $\sqrt{ }$ & & & $\sqrt{ }$ & $\sqrt{ }$ & $\sqrt{ }$ & $\sqrt{ }$ & 5.9 & 4.5 & 1 \\
\hline 17 & $\sqrt{ }$ & $\sqrt{ }$ & $\checkmark$ & $\sqrt{ }$ & & & & & $\sqrt{ }$ & $\sqrt{ }$ & & $\checkmark$ & 2.6 & 14.9 & 2 \\
\hline 18 & $\sqrt{ }$ & $\sqrt{ }$ & $\sqrt{ }$ & & $\sqrt{ }$ & & & & $\sqrt{ }$ & $\sqrt{ }$ & $\sqrt{ }$ & $\sqrt{ }$ & 2.4 & 5.9 & 2 \\
\hline 19 & $\sqrt{ }$ & & $\sqrt{ }$ & $\sqrt{ }$ & & $\sqrt{ }$ & & & $\sqrt{ }$ & $\sqrt{ }$ & $\sqrt{ }$ & $\sqrt{ }$ & - & - & - \\
\hline 20 & & & $\sqrt{ }$ & $\sqrt{ }$ & & & & & $\sqrt{ }$ & $\sqrt{ }$ & $\sqrt{ }$ & $\sqrt{ }$ & - & - & - \\
\hline 21 & $\sqrt{ }$ & $\sqrt{ }$ & & & $\sqrt{ }$ & & $\sqrt{ }$ & & $\sqrt{ }$ & $\sqrt{ }$ & $\checkmark$ & $\checkmark$ & 3.5 & 10.6 & 1 \\
\hline 22 & $\sqrt{ }$ & & $\sqrt{ }$ & & & $\sqrt{ }$ & & & $\sqrt{ }$ & $\sqrt{ }$ & $\sqrt{ }$ & $\sqrt{ }$ & - & - & - \\
\hline 23 & $\sqrt{ }$ & & $\ldots$ & & & & & & & & $\sqrt{ }$ & $\sqrt{ }$ & 4.0 & 2.5 & 5 \\
\hline 24 & $\sqrt{ }$ & $\sqrt{ }$ & 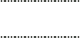 & $\sqrt{ }$ & & . & & & $\sqrt{ }$ & $\sqrt{ }$ & $\sqrt{ }$ & $\sqrt{ }$ & - & - & - \\
\hline 25 & $\sqrt{ }$ & $\sqrt{ }$ & $\sqrt{ }$ & & $\sqrt{ }$ & $\sqrt{ }$ & & & $\sqrt{ }$ & $\sqrt{ }$ & $\sqrt{ }$ & $\sqrt{ }$ & - & - & - \\
\hline 26 & $\sqrt{ }$ & $\sqrt{ }$ & $\sqrt{ }$ & $\sqrt{ }$ & & $\sqrt{ }$ & & & & $\sqrt{ }$ & $\sqrt{ }$ & $\checkmark$ & - & - & - \\
\hline 27 & $\checkmark$ & $\sqrt{ }$ & 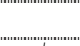 & & & & & & & & $\sqrt{ }$ & $\sqrt{ }$ & 2.9 & 4.5 & 3 \\
\hline 28 & $\sqrt{ }$ & $\sqrt{ }$ & $\sqrt{ }$ & & $\sqrt{ }$ & & $\sqrt{ }$ & & $\sqrt{ }$ & $\sqrt{ }$ & $\sqrt{ }$ & $\sqrt{ }$ & 8.8 & 13.9 & 1 \\
\hline 29 & $\sqrt{ }$ & $\sqrt{ }$ & $\sqrt{ }$ & & & & & & $\sqrt{ }$ & $\sqrt{ }$ & & $\sqrt{ }$ & 3.0 & 3.5 & 2 \\
\hline 30 & $\sqrt{ }$ & & $\sqrt{ }$ & $\sqrt{ }$ & $\sqrt{ }$ & & & & $\sqrt{ }$ & $\sqrt{ }$ & $\sqrt{ }$ & $\sqrt{ }$ & - & - & - \\
\hline 31 & $\sqrt{ }$ & $\sqrt{ }$ & $-\mathrm{ve}$ & & & & & & & $\sqrt{ }$ & $\sqrt{ }$ & $\sqrt{ }$ & 4.2 & 10.1 & 2 \\
\hline 32 & $\sqrt{ }$ & & & & & $\sqrt{ }$ & & & $\sqrt{ }$ & $\sqrt{ }$ & $\sqrt{ }$ & $\checkmark$ & - & - & - \\
\hline 33 & & $\sqrt{ }$ & $\sqrt{ }$ & & & & & & $\sqrt{ }$ & $\sqrt{ }$ & $\sqrt{ }$ & $\checkmark$ & - & - & - \\
\hline 34 & $\sqrt{ }$ & $\sqrt{ }$ & & $\sqrt{ }$ & $\sqrt{ }$ & $\sqrt{ }$ & & & $\sqrt{ }$ & $\sqrt{ }$ & $\sqrt{ }$ & $\sqrt{ }$ & - & - & - \\
\hline 35 & $\sqrt{ }$ & $\sqrt{ }$ & $\sqrt{ }$ & & & & & & & $\sqrt{ }$ & $\sqrt{ }$ & $\sqrt{ }$ & - & - & - \\
\hline 36 & $\sqrt{ }$ & & $\sqrt{ }$ & $\sqrt{ }$ & & & & & $\sqrt{ }$ & $\sqrt{ }$ & & $\sqrt{ }$ & 1.3 & 1.9 & 1 \\
\hline 37 & $\sqrt{ }$ & & & $\sqrt{ }$ & & & & & $\sqrt{ }$ & $\sqrt{ }$ & $\sqrt{ }$ & $\sqrt{ }$ & - & - & - \\
\hline 38 & $\sqrt{ }$ & $\sqrt{ }$ & $\sqrt{ }$ & $\checkmark$ & $\sqrt{ }$ & & $\checkmark$ & & $\sqrt{ }$ & $\sqrt{ }$ & $\checkmark$ & $\checkmark$ & 5.3 & 0.7 & 2 \\
\hline 39 & $\sqrt{ }$ & $\sqrt{ }$ & $\checkmark$ & $\sqrt{ }$ & $\sqrt{ }$ & $\sqrt{ }$ & $\sqrt{ }$ & & $\sqrt{ }$ & $\sqrt{ }$ & $\sqrt{ }$ & $\sqrt{ }$ & - & - & - \\
\hline 40 & $\sqrt{ }$ & & & $\sqrt{ }$ & & & & & & $\sqrt{ }$ & & $\sqrt{ }$ & 2.8 & -5.3 & 1 \\
\hline 41 & $\sqrt{ }$ & $\sqrt{ }$ & & & & & & & $\sqrt{ }$ & & $\sqrt{ }$ & $\sqrt{ }$ & 2.5 & 4.6 & 2 \\
\hline 42 & $\sqrt{ }$ & $\sqrt{ }$ & & & $\sqrt{ }$ & & $\sqrt{ }$ & & & $\sqrt{ }$ & $\sqrt{ }$ & $\checkmark$ & 4.6 & -1.0 & 1 \\
\hline 43 & $\sqrt{ }$ & $\sqrt{ }$ & & & $\sqrt{ }$ & & $\sqrt{ }$ & & $\sqrt{ }$ & & $\sqrt{ }$ & $\sqrt{ }$ & - & - & - \\
\hline 44 & $\sqrt{ }$ & $\sqrt{ }$ & $\sqrt{ }$ & $\sqrt{ }$ & & & $\sqrt{ }$ & & $\sqrt{ }$ & $\sqrt{ }$ & $\sqrt{ }$ & $\sqrt{ }$ & - & - & - \\
\hline 45 & $\sqrt{ }$ & $\sqrt{ }$ & & & $\sqrt{ }$ & & & & & & $\sqrt{ }$ & $\sqrt{ }$ & -0.5 & 21.3 & 1 \\
\hline 46 & $\sqrt{ }$ & & & & $\sqrt{ }$ & $\sqrt{ }$ & & & & $\sqrt{ }$ & $\sqrt{ }$ & $\sqrt{ }$ & - & - & - \\
\hline 47 & $\sqrt{ }$ & & $\sqrt{ }$ & $\sqrt{ }$ & $\sqrt{ }$ & $\sqrt{ }$ & & & $\sqrt{ }$ & $\sqrt{ }$ & $\sqrt{ }$ & $\sqrt{ }$ & 2.2 & 10.4 & 2 \\
\hline 48 & $\sqrt{ }$ & $\sqrt{ }$ & & & & & $\sqrt{ }$ & & & & $\sqrt{ }$ & $\checkmark$ & - & - & - \\
\hline 49 & $\sqrt{ }$ & $\sqrt{ }$ & $\sqrt{ }$ & $\sqrt{ }$ & & & & $\sqrt{ }$ & $\sqrt{ }$ & $\checkmark$ & $\checkmark$ & $\checkmark$ & - & - & - \\
\hline 50 & & & $\sqrt{ }$ & & & & & & $\sqrt{ }$ & $\sqrt{ }$ & $\checkmark$ & $\sqrt{ }$ & 2.1 & 2.8 & 1 \\
\hline
\end{tabular}


Table 52: Model's change in VTR prediction for worksites with Transit share between $5 \%$ to $15 \%$ and Vehicle Trip Rate between 90 to 100

\begin{tabular}{|c|c|c|c|c|c|c|c|c|c|c|c|c|c|c|c|}
\hline $\begin{array}{l}\text { Sr. } \\
\text { No. }\end{array}$ & $F \& A$ & ONS & CTB & FI N & CWW & FLEX & TELE & PMT & NONF & GRH & RSMP & MRKT & $\begin{array}{r}\text { Tra } \\
\text { [0. } \\
\text { VTR }\end{array}$ & $\begin{array}{l}\text { 1sit Sha } \\
05-0.15 \\
{[100-9}\end{array}$ & \\
\hline & & & & & & & & & & & & & Predicted & Actual & No.Ex \\
\hline 1 & $\sqrt{ }$ & $\sqrt{ }$ & $\sqrt{ }$ & $\sqrt{ }$ & & & & & $\sqrt{ }$ & $\sqrt{ }$ & $\sqrt{ }$ & $\sqrt{ }$ & -6.8 & 1.3 & 1 \\
\hline 2 & $\sqrt{ }$ & $\sqrt{ }$ & $\sqrt{ }$ & $\sqrt{ }$ & & $\sqrt{ }$ & & & $\sqrt{ }$ & $\sqrt{ }$ & $\sqrt{ }$ & $\sqrt{ }$ & -2.6 & -24.3 & 1 \\
\hline 3 & $\sqrt{ }$ & $\sqrt{ }$ & $\sqrt{ }$ & & & & & & $\sqrt{ }$ & $\sqrt{ }$ & $\sqrt{ }$ & $\sqrt{ }$ & -5.8 & 2.3 & 1 \\
\hline 4 & $\sqrt{ }$ & & $\sqrt{ }$ & $\sqrt{ }$ & & & & & $\sqrt{ }$ & $\sqrt{ }$ & $\sqrt{ }$ & $\sqrt{ }$ & -3.3 & -5.4 & 1 \\
\hline 5 & $\sqrt{ }$ & $\sqrt{ }$ & & & & & & & $\sqrt{ }$ & $\sqrt{ }$ & $\sqrt{ }$ & $\sqrt{ }$ & -4.6 & -1.3 & 2 \\
\hline 6 & & $\sqrt{ }$ & $\sqrt{ }$ & $\sqrt{ }$ & & & & & $\sqrt{ }$ & $\sqrt{ }$ & $\sqrt{ }$ & $\sqrt{ }$ & -7.5 & 4.0 & 3 \\
\hline 7 & $\sqrt{ }$ & $\sqrt{ }$ & & & & $\sqrt{ }$ & & & $\sqrt{ }$ & $\sqrt{ }$ & $\sqrt{ }$ & $\sqrt{ }$ & -1.8 & -10.1 & 2 \\
\hline 8 & $\sqrt{ }$ & $\sqrt{ }$ & $\sqrt{ }$ & $\sqrt{ }$ & $\sqrt{ }$ & & & & $\sqrt{ }$ & $\sqrt{ }$ & $\sqrt{ }$ & $\sqrt{ }$ & - & - & - \\
\hline 9 & $\sqrt{ }$ & & $\sqrt{ }$ & & & & & & $\sqrt{ }$ & $\sqrt{ }$ & $\sqrt{ }$ & $\sqrt{ }$ & -5.4 & -3.1 & 2 \\
\hline 10 & $\sqrt{ }$ & $\sqrt{ }$ & & & $\sqrt{ }$ & & & & $\sqrt{ }$ & $\sqrt{ }$ & $\sqrt{ }$ & $\sqrt{ }$ & - & - & - \\
\hline 11 & $\sqrt{ }$ & & $\sqrt{ }$ & $\sqrt{ }$ & & & & & & $\sqrt{ }$ & $\sqrt{ }$ & $\sqrt{ }$ & -3.3 & -5.4 & 1 \\
\hline 12 & $\sqrt{ }$ & & & & & & & & & $\sqrt{ }$ & $\sqrt{ }$ & $\sqrt{ }$ & - & - & - \\
\hline 13 & $\sqrt{ }$ & $\sqrt{ }$ & $\sqrt{ }$ & & & $\sqrt{ }$ & & & $\sqrt{ }$ & $\sqrt{ }$ & $\sqrt{ }$ & $\sqrt{ }$ & - & - & - \\
\hline 14 & $\sqrt{ }$ & $\sqrt{ }$ & $\sqrt{ }$ & $\sqrt{ }$ & & & & & & $\sqrt{ }$ & $\sqrt{ }$ & $\sqrt{ }$ & - & - & - \\
\hline 15 & $\sqrt{ }$ & - & & & & & & & $\sqrt{ }$ & $\sqrt{ }$ & $\sqrt{ }$ & $\sqrt{ }$ & - & - & - \\
\hline 16 & $\sqrt{ }$ & $\sqrt{ }$ & $\sqrt{ }$ & $\sqrt{ }$ & $\sqrt{ }$ & $\sqrt{ }$ & & & $\sqrt{ }$ & $\sqrt{ }$ & $\sqrt{ }$ & $\sqrt{ }$ & - & - & - \\
\hline 17 & $\sqrt{ }$ & $\sqrt{ }$ & $\sqrt{ }$ & $\sqrt{ }$ & & & & & $\sqrt{ }$ & $\sqrt{ }$ & & $\sqrt{ }$ & - & - & - \\
\hline 18 & $\sqrt{ }$ & $\sqrt{ }$ & $\sqrt{ }$ & . & $\sqrt{ }$ & & & & $\sqrt{ }$ & $\sqrt{ }$ & $\sqrt{ }$ & $\sqrt{ }$ & - & - & - \\
\hline 19 & $\sqrt{ }$ & & $\sqrt{ }$ & $\sqrt{ }$ & & $\sqrt{ }$ & & & $\sqrt{ }$ & $\sqrt{ }$ & $\sqrt{ }$ & $\sqrt{ }$ & - & - & - \\
\hline 20 & & & $\sqrt{ }$ & $\sqrt{ }$ & & & & & $\sqrt{ }$ & $\sqrt{ }$ & $\sqrt{ }$ & $\sqrt{ }$ & - & - & - \\
\hline 21 & $\sqrt{ }$ & $\sqrt{ }$ & & & $\sqrt{ }$ & & $\sqrt{ }$ & & $\sqrt{ }$ & $\sqrt{ }$ & $\sqrt{ }$ & $\sqrt{ }$ & - & - & - \\
\hline 22 & $\sqrt{ }$ & & $\sqrt{ }$ & & & $\sqrt{ }$ & & & $\sqrt{ }$ & $\sqrt{ }$ & $\sqrt{ }$ & $\sqrt{ }$ & - & - & - \\
\hline 23 & $\sqrt{ }$ & & & & & & & & & & $\sqrt{ }$ & $\sqrt{ }$ & - & - & - \\
\hline 24 & $\sqrt{ }$ & $\sqrt{ }$ & & $\sqrt{ }$ & & & & & $\sqrt{ }$ & $\sqrt{ }$ & $\sqrt{ }$ & $\sqrt{ }$ & - & - & - \\
\hline 25 & $\sqrt{ }$ & $\sqrt{ }$ & $\sqrt{ }$ & & $\sqrt{ }$ & $\sqrt{ }$ & & & $\sqrt{ }$ & $\sqrt{ }$ & $\sqrt{ }$ & $\sqrt{ }$ & - & - & - \\
\hline 26 & $\sqrt{ }$ & $\sqrt{ }$ & $\sqrt{ }$ & $\sqrt{ }$ & & $\sqrt{ }$ & & & & $\sqrt{ }$ & $\sqrt{ }$ & $\sqrt{ }$ & - & - & - \\
\hline 27 & $\sqrt{ }$ & $\sqrt{ }$ & - & & & & & & & & $\sqrt{ }$ & $\sqrt{ }$ & 0.5 & -5.0 & 3 \\
\hline 28 & $\sqrt{ }$ & $\sqrt{ }$ & $\sqrt{ }$ & & $\sqrt{ }$ & & $\sqrt{ }$ & & $\sqrt{ }$ & $\sqrt{ }$ & $\sqrt{ }$ & $\sqrt{ }$ & - & - & - \\
\hline 29 & $\sqrt{ }$ & $\sqrt{ }$ & $\sqrt{ }$ & & & & & & $\sqrt{ }$ & $\sqrt{ }$ & 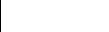 & $\sqrt{ }$ & - & - & - \\
\hline 30 & $\sqrt{ }$ & . & $\sqrt{ }$ & $\sqrt{ }$ & $\sqrt{ }$ & & & & $\sqrt{ }$ & $\sqrt{ }$ & $\sqrt{ }$ & $\sqrt{ }$ & - & - & - \\
\hline 31 & $\sqrt{ }$ & $\sqrt{ }$ & & & & & & & & $\sqrt{ }$ & $\sqrt{ }$ & $\sqrt{ }$ & - & - & - \\
\hline 32 & $\sqrt{ }$ & . & & & & $\sqrt{ }$ & & & $\sqrt{ }$ & $\sqrt{ }$ & $\sqrt{ }$ & $\sqrt{ }$ & - & - & - \\
\hline 33 & & $\sqrt{ }$ & $\sqrt{ }$ & & & & & & $\sqrt{ }$ & $\sqrt{ }$ & $\sqrt{ }$ & $\sqrt{ }$ & - & - & - \\
\hline 34 & $\sqrt{ }$ & $\sqrt{ }$ & & $\sqrt{ }$ & $\sqrt{ }$ & $\sqrt{ }$ & & & $\sqrt{ }$ & $\sqrt{ }$ & $\sqrt{ }$ & $\sqrt{ }$ & - & - & - \\
\hline 35 & $\sqrt{ }$ & $\sqrt{ }$ & $\sqrt{ }$ & & & & & & & $\sqrt{ }$ & $\sqrt{ }$ & $\sqrt{ }$ & - & - & - \\
\hline 36 & $\sqrt{ }$ & & $\sqrt{ }$ & $\sqrt{ }$ & & & & & $\sqrt{ }$ & $\sqrt{ }$ & & $\sqrt{ }$ & -7.6 & -0.9 & 1 \\
\hline 37 & $\sqrt{ }$ & & & $\sqrt{ }$ & & & & & $\sqrt{ }$ & $\sqrt{ }$ & $\sqrt{ }$ & $\sqrt{ }$ & - & - & - \\
\hline 38 & $\sqrt{ }$ & $\sqrt{ }$ & $\sqrt{ }$ & $\sqrt{ }$ & $\sqrt{ }$ & & $\sqrt{ }$ & & $\sqrt{ }$ & $\sqrt{ }$ & $\sqrt{ }$ & $\sqrt{ }$ & - & - & - \\
\hline 39 & $\sqrt{ }$ & $\sqrt{ }$ & $\sqrt{ }$ & $\sqrt{ }$ & $\sqrt{ }$ & $\sqrt{ }$ & $\sqrt{ }$ & & $\sqrt{ }$ & $\sqrt{ }$ & $\sqrt{ }$ & $\sqrt{ }$ & - & - & - \\
\hline 40 & $\sqrt{ }$ & & & $\sqrt{ }$ & & & & & & $\sqrt{ }$ & & $\sqrt{ }$ & - & - & - \\
\hline 41 & $\sqrt{ }$ & $\sqrt{ }$ & & & & & & & $\sqrt{ }$ & & $\sqrt{ }$ & $\sqrt{ }$ & -2.9 & -9.9 & 1 \\
\hline 42 & $\sqrt{ }$ & $\sqrt{ }$ & & & $\sqrt{ }$ & & $\sqrt{ }$ & & & $\sqrt{ }$ & $\sqrt{ }$ & $\sqrt{ }$ & - & - & - \\
\hline 43 & $\sqrt{ }$ & $\sqrt{ }$ & & & $\sqrt{ }$ & & $\sqrt{ }$ & & $\sqrt{ }$ & & $\sqrt{ }$ & $\sqrt{ }$ & - & - & - \\
\hline 44 & $\sqrt{ }$ & $\sqrt{ }$ & $\sqrt{ }$ & $\sqrt{ }$ & & & $\sqrt{ }$ & & $\sqrt{ }$ & $\sqrt{ }$ & $\sqrt{ }$ & V & - & - & - \\
\hline 45 & $\sqrt{ }$ & $\sqrt{ }$ & & & $\sqrt{ }$ & & & & & & $\sqrt{ }$ & $\sqrt{ }$ & - & - & - \\
\hline 46 & $\sqrt{ }$ & & & & $\sqrt{ }$ & $\sqrt{ }$ & & & & $\sqrt{ }$ & $\sqrt{ }$ & $\sqrt{ }$ & - & - & - \\
\hline 47 & $\sqrt{ }$ & & $\sqrt{ }$ & $\sqrt{ }$ & $\sqrt{ }$ & $\sqrt{ }$ & & & $\sqrt{ }$ & $\sqrt{ }$ & $\sqrt{ }$ & $\sqrt{ }$ & - & - & - \\
\hline 48 & $\sqrt{ }$ & $\sqrt{ }$ & & & & & $\sqrt{ }$ & & & & $\sqrt{ }$ & $\sqrt{ }$ & - & - & - \\
\hline 49 & $\sqrt{ }$ & $\sqrt{ }$ & $\sqrt{ }$ & $\sqrt{ }$ & & & & $\sqrt{ }$ & $\sqrt{ }$ & $\sqrt{ }$ & $\sqrt{ }$ & $\sqrt{ }$ & - & - & - \\
\hline 50 & & & $\sqrt{ }$ & & & & & & $\sqrt{ }$ & $\sqrt{ }$ & $\sqrt{ }$ & $\sqrt{ }$ & -4.9 & -11.0 & 1 \\
\hline
\end{tabular}


Table 53: Model's change in VTR prediction for worksites with Transit share between $5 \%$ to $15 \%$ and Vehicle Trip Rate between 80 to 90

\begin{tabular}{|c|c|c|c|c|c|c|c|c|c|c|c|c|c|c|c|}
\hline $\begin{array}{l}\text { Sr. } \\
\text { No. }\end{array}$ & $F \& A$ & ONS & СТВ & FI N & CWW & FLEX & TELE & PMT & NONF & GRH & RSMP & MRKT & $\begin{array}{c}\text { Trar } \\
{[0 .} \\
\text { VTR } \\
\end{array}$ & $\begin{array}{l}\text { nsit Sha } \\
05-0.15 \\
-[90-80\end{array}$ & \\
\hline & & & & & & & & & & & & & Predicted & Actual & No.Ex \\
\hline 1 & $\sqrt{ }$ & $\sqrt{ }$ & $\checkmark$ & $\checkmark$ & & & & & $\sqrt{ }$ & $\sqrt{ }$ & $\sqrt{ }$ & $\sqrt{ }$ & -3.2 & -3.7 & 44 \\
\hline 2 & $\checkmark$ & $\sqrt{ }$ & $\sqrt{ }$ & $\sqrt{ }$ & & $\sqrt{ }$ & & & $\sqrt{ }$ & $\sqrt{ }$ & $\sqrt{ }$ & $\sqrt{ }$ & -1.1 & -1.1 & 27 \\
\hline 3 & $\sqrt{ }$ & $\sqrt{ }$ & $\sqrt{ }$ & & & & & & $\sqrt{ }$ & $\sqrt{ }$ & $\sqrt{ }$ & $\sqrt{ }$ & -4.5 & -2.6 & 26 \\
\hline 4 & $\sqrt{ }$ & & $\sqrt{ }$ & $\sqrt{ }$ & & & & & $\sqrt{ }$ & $\sqrt{ }$ & $\sqrt{ }$ & $\sqrt{ }$ & -4.3 & -5.7 & 14 \\
\hline 5 & $\sqrt{ }$ & $\sqrt{ }$ & & & & & & & $\sqrt{ }$ & $\sqrt{ }$ & $\sqrt{ }$ & $\sqrt{ }$ & -1.9 & -3.7 & 20 \\
\hline 6 & & $\sqrt{ }$ & $\sqrt{ }$ & $\checkmark$ & & & & & $\sqrt{ }$ & $\checkmark$ & $\sqrt{ }$ & $\checkmark$ & -3.7 & 0.0 & 17 \\
\hline 7 & $\sqrt{ }$ & $\sqrt{ }$ & & & & $\sqrt{ }$ & & & $\sqrt{ }$ & $\sqrt{ }$ & $\sqrt{ }$ & $\sqrt{ }$ & -1.5 & -0.7 & 13 \\
\hline 8 & $\sqrt{ }$ & $\sqrt{ }$ & $\checkmark$ & $\sqrt{ }$ & $\sqrt{ }$ & & & & $\sqrt{ }$ & $\sqrt{ }$ & $\sqrt{ }$ & $\sqrt{ }$ & 0.5 & -17.2 & 3 \\
\hline 9 & $\sqrt{ }$ & & $\sqrt{ }$ & & & & & & $\sqrt{ }$ & $\sqrt{ }$ & $\sqrt{ }$ & $\sqrt{ }$ & -4.5 & -4.8 & 10 \\
\hline 10 & $\sqrt{ }$ & $\sqrt{ }$ & & & $\sqrt{ }$ & & & & $\sqrt{ }$ & $\sqrt{ }$ & $\sqrt{ }$ & $\sqrt{ }$ & -1.2 & 0.0 & 7 \\
\hline 11 & $\sqrt{ }$ & & $\sqrt{ }$ & $\checkmark$ & & & & & & $\sqrt{ }$ & $\sqrt{ }$ & $\sqrt{ }$ & -4.3 & -5.7 & 14 \\
\hline 12 & $\sqrt{ }$ & & & & & & & & & $\sqrt{ }$ & $\sqrt{ }$ & $\sqrt{ }$ & -2.4 & 0.2 & 8 \\
\hline 13 & $\checkmark$ & $\sqrt{ }$ & $\sqrt{ }$ & & & $\sqrt{ }$ & & & $\sqrt{ }$ & $\sqrt{ }$ & $\sqrt{ }$ & $\sqrt{ }$ & -2.3 & -3.7 & 14 \\
\hline 14 & $\sqrt{ }$ & $\sqrt{ }$ & $\sqrt{ }$ & $\sqrt{ }$ & & & & & & $\sqrt{ }$ & $\sqrt{ }$ & $\sqrt{ }$ & -2.7 & -2.6 & 15 \\
\hline 15 & $\sqrt{ }$ & & & & & & & & $\sqrt{ }$ & $\sqrt{ }$ & $\sqrt{ }$ & $\sqrt{ }$ & -3.4 & -0.1 & 12 \\
\hline 16 & $\sqrt{ }$ & $\sqrt{ }$ & $\sqrt{ }$ & $\checkmark$ & $\sqrt{ }$ & $\sqrt{ }$ & & & $\sqrt{ }$ & $\sqrt{ }$ & $\sqrt{ }$ & $\checkmark$ & 0.5 & -2.0 & 2 \\
\hline 17 & $\sqrt{ }$ & $\sqrt{ }$ & $\checkmark$ & $\checkmark$ & & & & & $\sqrt{ }$ & $\sqrt{ }$ & & $\checkmark$ & -3.9 & 0.5 & 13 \\
\hline 18 & $\sqrt{ }$ & $\sqrt{ }$ & $\sqrt{ }$ & & $\sqrt{ }$ & & & & $\sqrt{ }$ & $\sqrt{ }$ & $\sqrt{ }$ & $\sqrt{ }$ & -1.8 & -3.8 & 5 \\
\hline 19 & $\sqrt{ }$ & & $\sqrt{ }$ & $\sqrt{ }$ & & $\sqrt{ }$ & & & $\sqrt{ }$ & $\sqrt{ }$ & $\sqrt{ }$ & $\sqrt{ }$ & -1.6 & -4.7 & 6 \\
\hline 20 & & & $\sqrt{ }$ & $\sqrt{ }$ & & & & & $\sqrt{ }$ & $\sqrt{ }$ & $\sqrt{ }$ & $\sqrt{ }$ & -4.4 & -3.0 & 13 \\
\hline 21 & $\sqrt{ }$ & $\sqrt{ }$ & & & $\checkmark$ & & $\sqrt{ }$ & & $\sqrt{ }$ & $\checkmark$ & $\sqrt{ }$ & $\sqrt{ }$ & 0.1 & -2.8 & 6 \\
\hline 22 & $\sqrt{ }$ & & $\checkmark$ & & & $\sqrt{ }$ & & & $\sqrt{ }$ & $\sqrt{ }$ & $\sqrt{ }$ & $\sqrt{ }$ & -3.7 & -3.4 & 5 \\
\hline 23 & $\sqrt{ }$ & & & & & & & & & & $\sqrt{ }$ & $\checkmark$ & -4.8 & -2.4 & 3 \\
\hline 24 & $\sqrt{ }$ & $\sqrt{ }$ & & $\sqrt{ }$ & & & & & $\sqrt{ }$ & $\sqrt{ }$ & $\sqrt{ }$ & $\sqrt{ }$ & -1.5 & -1.5 & 1 \\
\hline 25 & $\sqrt{ }$ & $\sqrt{ }$ & $\sqrt{ }$ & & $\sqrt{ }$ & $\sqrt{ }$ & & & $\sqrt{ }$ & $\sqrt{ }$ & $\sqrt{ }$ & $\sqrt{ }$ & 0.0 & -0.5 & 6 \\
\hline 26 & $\sqrt{ }$ & $\sqrt{ }$ & $\sqrt{ }$ & $\sqrt{ }$ & & $\sqrt{ }$ & & & & $\sqrt{ }$ & $\sqrt{ }$ & $\checkmark$ & -3.2 & 1.4 & 8 \\
\hline 27 & $\sqrt{ }$ & $\sqrt{ }$ & & & & & & & & & $\sqrt{ }$ & $\sqrt{ }$ & -2.8 & -3.6 & 21 \\
\hline 28 & $\sqrt{ }$ & $\sqrt{ }$ & $\checkmark$ & & $\checkmark$ & & $\sqrt{ }$ & & $\sqrt{ }$ & $\sqrt{ }$ & $\sqrt{ }$ & $\sqrt{ }$ & -1.1 & -0.3 & 4 \\
\hline 29 & $\sqrt{ }$ & $\sqrt{ }$ & $\checkmark$ & & & & & & $\sqrt{ }$ & $\sqrt{ }$ & & $\sqrt{ }$ & -3.6 & -5.6 & 1 \\
\hline 30 & $\sqrt{ }$ & & $\sqrt{ }$ & $\sqrt{ }$ & $\sqrt{ }$ & & & & $\sqrt{ }$ & $\sqrt{ }$ & $\sqrt{ }$ & $\sqrt{ }$ & -2.0 & 0.0 & 7 \\
\hline 31 & $\sqrt{ }$ & $\sqrt{ }$ & - & & & & & & & $\sqrt{ }$ & $\sqrt{ }$ & $\sqrt{ }$ & -1.2 & -3.6 & 3 \\
\hline 32 & $\sqrt{ }$ & & & & & $\sqrt{ }$ & & & $\sqrt{ }$ & $\sqrt{ }$ & $\sqrt{ }$ & $\checkmark$ & -1.6 & -0.4 & 5 \\
\hline 33 & & $\sqrt{ }$ & $\checkmark$ & & & & & & $\sqrt{ }$ & $\sqrt{ }$ & $\sqrt{ }$ & $\checkmark$ & -3.7 & -1.1 & 10 \\
\hline 34 & $\checkmark$ & $\sqrt{ }$ & & $\checkmark$ & $\sqrt{ }$ & $\sqrt{ }$ & & & $\sqrt{ }$ & $\sqrt{ }$ & $\checkmark$ & $\sqrt{ }$ & -0.4 & 0.8 & 6 \\
\hline 35 & $\sqrt{ }$ & $\sqrt{ }$ & $\sqrt{ }$ & & & & & & & $\sqrt{ }$ & $\sqrt{ }$ & $\sqrt{ }$ & -2.6 & -2.0 & 7 \\
\hline 36 & $\sqrt{ }$ & & $\sqrt{ }$ & $\checkmark$ & & & & & $\sqrt{ }$ & $\sqrt{ }$ & & $\sqrt{ }$ & -3.8 & -5.5 & 4 \\
\hline 37 & $\sqrt{ }$ & & & $\checkmark$ & & & & & $\sqrt{ }$ & $\sqrt{ }$ & $\sqrt{ }$ & $\sqrt{ }$ & -3.5 & -5.7 & 2 \\
\hline 38 & $\checkmark$ & $\sqrt{ }$ & $\checkmark$ & $\checkmark$ & $\checkmark$ & & $\checkmark$ & & $\sqrt{ }$ & $\sqrt{ }$ & $\checkmark$ & $\checkmark$ & 0.8 & -4.9 & 3 \\
\hline 39 & $\sqrt{ }$ & $\sqrt{ }$ & $\checkmark$ & $\checkmark$ & $\checkmark$ & $\sqrt{ }$ & $\sqrt{ }$ & & $\sqrt{ }$ & $\sqrt{ }$ & $\sqrt{ }$ & $\sqrt{ }$ & -0.9 & 2.0 & 5 \\
\hline 40 & $\sqrt{ }$ & & & $\sqrt{ }$ & & & & & & $\sqrt{ }$ & & $\sqrt{ }$ & -2.5 & -1.4 & 3 \\
\hline 41 & $\sqrt{ }$ & $\sqrt{ }$ & & & & & & & $\sqrt{ }$ & & $\sqrt{ }$ & $\sqrt{ }$ & -1.4 & 4.6 & 3 \\
\hline 42 & $\sqrt{ }$ & $\sqrt{ }$ & & & $\checkmark$ & & $\sqrt{ }$ & & & $\sqrt{ }$ & $\sqrt{ }$ & $\sqrt{ }$ & -2.9 & 1.2 & 2 \\
\hline 43 & $\sqrt{ }$ & $\sqrt{ }$ & & & $\checkmark$ & & $\sqrt{ }$ & & $\sqrt{ }$ & & $\sqrt{ }$ & $\sqrt{ }$ & 0.9 & -3.8 & 6 \\
\hline 44 & $\sqrt{ }$ & $\sqrt{ }$ & $\sqrt{ }$ & $\sqrt{ }$ & & & $\sqrt{ }$ & & $\sqrt{ }$ & $\sqrt{ }$ & $\sqrt{ }$ & $\sqrt{ }$ & -3.7 & -7.4 & 4 \\
\hline 45 & $\sqrt{ }$ & $\sqrt{ }$ & & & $\sqrt{ }$ & & & & & & $\sqrt{ }$ & $\sqrt{ }$ & 0.1 & -0.1 & 4 \\
\hline 46 & $\sqrt{ }$ & & & & $\sqrt{ }$ & $\sqrt{ }$ & & & & $\sqrt{ }$ & $\sqrt{ }$ & $\sqrt{ }$ & -1.8 & -1.4 & 1 \\
\hline 47 & $\sqrt{ }$ & & $\checkmark$ & $\sqrt{ }$ & $\sqrt{ }$ & $\sqrt{ }$ & & & $\sqrt{ }$ & $\sqrt{ }$ & $\sqrt{ }$ & $\sqrt{ }$ & -1.1 & -9.4 & 2 \\
\hline 48 & $\sqrt{ }$ & $V$ & & & & & $\sqrt{ }$ & & & & $\sqrt{ }$ & $\checkmark$ & -3.6 & 0.5 & 4 \\
\hline 49 & $\sqrt{ }$ & $\sqrt{ }$ & $\checkmark$ & $\checkmark$ & & & & $\sqrt{ }$ & $\sqrt{ }$ & $\checkmark$ & $\sqrt{ }$ & $\checkmark$ & -3.5 & -3.0 & 3 \\
\hline 50 & & & $\sqrt{ }$ & & & & & & $\sqrt{ }$ & $\sqrt{ }$ & $\checkmark$ & $\sqrt{ }$ & -4.4 & -0.2 & 7 \\
\hline
\end{tabular}


Table 54: Model's change in VTR prediction for worksites with Transit share between $5 \%$ to $15 \%$ and Vehicle Trip Rate between 70 to 80

\begin{tabular}{|c|c|c|c|c|c|c|c|c|c|c|c|c|c|c|c|}
\hline $\begin{array}{l}\text { Sr. } \\
\text { No. }\end{array}$ & $F \& A$ & ONS & СТВ & FI N & CWW & FLEX & TELE & PMT & NONF & GRH & RSMP & MRKT & $\begin{array}{r}\text { Tran } \\
{\left[0.0^{-1}\right.} \\
\text { VTR } \\
\end{array}$ & $\begin{array}{l}\text { nsit Sha } \\
05-0.15 \\
-[80-70\end{array}$ & \\
\hline & & & & & & & & & & & & & Predicted & Actual & No.Ex \\
\hline 1 & $\sqrt{ }$ & $\sqrt{ }$ & $\checkmark$ & $\checkmark$ & & & & & $\sqrt{ }$ & $\sqrt{ }$ & $\sqrt{ }$ & $\sqrt{ }$ & -1.5 & -0.2 & 62 \\
\hline 2 & $\sqrt{ }$ & $\sqrt{ }$ & $\sqrt{ }$ & $\sqrt{ }$ & & $\sqrt{ }$ & & & $\sqrt{ }$ & $\sqrt{ }$ & $\sqrt{ }$ & $\sqrt{3}$ & -0.2 & -1.5 & 24 \\
\hline 3 & $\sqrt{ }$ & $\sqrt{ }$ & $\sqrt{ }$ & & & & & & $\sqrt{ }$ & $\sqrt{ }$ & $\sqrt{ }$ & $\sqrt{ }$ & -1.4 & 0.6 & 54 \\
\hline 4 & $\sqrt{ }$ & & $\sqrt{ }$ & $\sqrt{ }$ & & & & & $\sqrt{ }$ & $\sqrt{ }$ & $\sqrt{ }$ & $\sqrt{ }$ & -2.2 & -1.4 & 28 \\
\hline 5 & $\sqrt{ }$ & $\sqrt{ }$ & & & & & & & $\sqrt{ }$ & $\sqrt{ }$ & $\sqrt{ }$ & $\sqrt{ }$ & 0.1 & 0.1 & 33 \\
\hline 6 & & $\sqrt{ }$ & $\sqrt{ }$ & $\checkmark$ & & & & & $\sqrt{ }$ & $\checkmark$ & $\sqrt{ }$ & $\sqrt{ }$ & -1.0 & 0.5 & 52 \\
\hline 7 & $\sqrt{ }$ & $\sqrt{ }$ & & & & $\sqrt{ }$ & & & $\sqrt{ }$ & $\sqrt{ }$ & $\sqrt{ }$ & $\sqrt{3}$ & -0.7 & 0.0 & 20 \\
\hline 8 & $\sqrt{ }$ & $\sqrt{ }$ & $\checkmark$ & $\sqrt{ }$ & $\sqrt{ }$ & & & & $\sqrt{ }$ & $\sqrt{ }$ & $\sqrt{ }$ & $\sqrt{ }$ & 1.1 & 0.3 & 11 \\
\hline 9 & $\sqrt{ }$ & & $\sqrt{ }$ & & & & & & $\sqrt{ }$ & $\sqrt{ }$ & $\sqrt{ }$ & $\sqrt{ }$ & -2.5 & 0.2 & 30 \\
\hline 10 & $\sqrt{ }$ & $\sqrt{ }$ & & & $\sqrt{ }$ & & & & $\sqrt{ }$ & $\sqrt{ }$ & $\sqrt{ }$ & $\sqrt{ }$ & 0.8 & 0.6 & 29 \\
\hline 11 & $\sqrt{ }$ & & $\sqrt{ }$ & $\sqrt{ }$ & & & & & & $\sqrt{ }$ & $\sqrt{ }$ & $\sqrt{ }$ & -2.2 & -1.4 & 28 \\
\hline 12 & $\sqrt{ }$ & & & & & & & & & $\sqrt{ }$ & $\sqrt{ }$ & $\sqrt{ }$ & -0.2 & 1.6 & 18 \\
\hline 13 & $\checkmark$ & $\sqrt{ }$ & $\checkmark$ & & & $\sqrt{ }$ & & & $\sqrt{ }$ & $\sqrt{ }$ & $\checkmark$ & $\checkmark$ & -1.1 & -2.6 & 14 \\
\hline 14 & $\sqrt{ }$ & $\sqrt{ }$ & $\sqrt{ }$ & $\sqrt{ }$ & & & & & & $\checkmark$ & $\sqrt{ }$ & $\checkmark$ & -1.4 & -0.8 & 18 \\
\hline 15 & $\sqrt{ }$ & & & & & & & & $\sqrt{ }$ & $\sqrt{ }$ & $\sqrt{ }$ & $\sqrt{ }$ & -0.6 & -0.5 & 9 \\
\hline 16 & $\sqrt{ }$ & $\sqrt{ }$ & $\sqrt{ }$ & $\checkmark$ & $\sqrt{ }$ & $\sqrt{ }$ & & & $\sqrt{ }$ & $\sqrt{ }$ & $\sqrt{ }$ & $\checkmark$ & 1.4 & 1.7 & 12 \\
\hline 17 & $\sqrt{ }$ & $\sqrt{ }$ & $\checkmark$ & $\checkmark$ & & & & & $\sqrt{ }$ & $\sqrt{ }$ & & $\checkmark$ & -2.2 & 2.1 & 16 \\
\hline 18 & $\sqrt{ }$ & $\sqrt{ }$ & $\sqrt{ }$ & & $\sqrt{ }$ & & & & $\sqrt{ }$ & $\sqrt{ }$ & $\sqrt{ }$ & $\sqrt{ }$ & 0.2 & 0.6 & 15 \\
\hline 19 & $\sqrt{ }$ & & $\sqrt{ }$ & $\sqrt{ }$ & & $\sqrt{ }$ & & & $\sqrt{ }$ & $\sqrt{ }$ & $\sqrt{ }$ & $\sqrt{ }$ & -1.8 & 0.7 & 6 \\
\hline 20 & & & $\sqrt{ }$ & $\sqrt{ }$ & & & & & $\sqrt{ }$ & $\sqrt{ }$ & $\sqrt{ }$ & $\sqrt{ }$ & -2.1 & 0.3 & 18 \\
\hline 21 & $\sqrt{ }$ & $\sqrt{ }$ & & & $\checkmark$ & & $\sqrt{ }$ & & $\sqrt{ }$ & $\checkmark$ & $\sqrt{ }$ & $\sqrt{ }$ & 1.7 & -1.1 & 13 \\
\hline 22 & $\sqrt{ }$ & & $\checkmark$ & & & $\sqrt{ }$ & & & $\sqrt{ }$ & $\sqrt{ }$ & $\sqrt{ }$ & $\sqrt{ }$ & -3.2 & -1.5 & 13 \\
\hline 23 & $\sqrt{ }$ & & & & & & & & & & $\sqrt{ }$ & $\sqrt{ }$ & -1.3 & -0.6 & 7 \\
\hline 24 & $\sqrt{ }$ & $\sqrt{ }$ & & $\sqrt{ }$ & & & & & $\sqrt{ }$ & $\sqrt{ }$ & $\sqrt{ }$ & $\sqrt{ }$ & -0.2 & -3.4 & 9 \\
\hline 25 & $\sqrt{ }$ & $\sqrt{ }$ & $\sqrt{ }$ & & $\sqrt{ }$ & $\sqrt{ }$ & & & $\sqrt{ }$ & $\sqrt{ }$ & $\sqrt{ }$ & $\sqrt{ }$ & 1.1 & 0.1 & 7 \\
\hline 26 & $\sqrt{ }$ & $\sqrt{ }$ & $\sqrt{ }$ & $\sqrt{ }$ & & $\sqrt{ }$ & & & & $\sqrt{ }$ & $\sqrt{ }$ & $\checkmark$ & 0.8 & 0.8 & 5 \\
\hline 27 & $\sqrt{ }$ & $\sqrt{ }$ & & & & & & & & & $\sqrt{ }$ & $\sqrt{ }$ & -0.7 & -1.2 & 3 \\
\hline 28 & $\sqrt{ }$ & $\sqrt{ }$ & $\checkmark$ & & $\checkmark$ & & $\sqrt{ }$ & & $\sqrt{ }$ & $\sqrt{ }$ & $\sqrt{ }$ & $\sqrt{ }$ & 0.2 & 3.3 & 6 \\
\hline 29 & $\sqrt{ }$ & $\sqrt{ }$ & $\checkmark$ & & & & & & $\sqrt{ }$ & $\sqrt{ }$ & & $\sqrt{ }$ & -0.9 & 0.8 & 14 \\
\hline 30 & $\sqrt{ }$ & & $\sqrt{ }$ & $\sqrt{ }$ & $\sqrt{ }$ & & & & $\sqrt{ }$ & $\sqrt{ }$ & $\sqrt{ }$ & $\sqrt{ }$ & -0.2 & -3.5 & 5 \\
\hline 31 & $\sqrt{ }$ & $\sqrt{ }$ & - & & & & & & & $\sqrt{ }$ & $\sqrt{ }$ & $\sqrt{ }$ & 1.7 & 2.9 & 2 \\
\hline 32 & $\sqrt{ }$ & & & & & $\sqrt{ }$ & & & $\sqrt{ }$ & $\sqrt{ }$ & $\sqrt{ }$ & $\sqrt{ }$ & -1.4 & 3.8 & 11 \\
\hline 33 & & $\sqrt{ }$ & $\checkmark$ & & & & & & $\sqrt{ }$ & $\sqrt{ }$ & $\sqrt{ }$ & $\checkmark$ & 0.1 & -3.0 & 9 \\
\hline 34 & $\checkmark$ & $\sqrt{ }$ & & $\sqrt{ }$ & $\sqrt{ }$ & $\sqrt{ }$ & & & $\sqrt{ }$ & $\sqrt{ }$ & $\checkmark$ & $\sqrt{ }$ & 1.1 & -1.6 & 9 \\
\hline 35 & $\sqrt{ }$ & $\sqrt{ }$ & $\sqrt{ }$ & & & & & & & $\sqrt{ }$ & $\sqrt{ }$ & $\sqrt{ }$ & -0.9 & 1.1 & 12 \\
\hline 36 & $\sqrt{ }$ & & $\sqrt{ }$ & $\checkmark$ & & & & & $\sqrt{ }$ & $\sqrt{ }$ & & $\sqrt{ }$ & -0.3 & -5.5 & 3 \\
\hline 37 & $\sqrt{ }$ & & & $\checkmark$ & & & & & $\sqrt{ }$ & $\sqrt{ }$ & $\sqrt{ }$ & $\sqrt{ }$ & 1.6 & -0.6 & 2 \\
\hline 38 & $\checkmark$ & $\sqrt{ }$ & $\checkmark$ & $\checkmark$ & $\checkmark$ & & $\checkmark$ & & $\sqrt{ }$ & $\sqrt{ }$ & $\checkmark$ & $\checkmark$ & 3.4 & -1.5 & 4 \\
\hline 39 & $\sqrt{ }$ & $\sqrt{ }$ & $\checkmark$ & $\checkmark$ & $\checkmark$ & $\sqrt{ }$ & $\sqrt{ }$ & & $\sqrt{ }$ & $\sqrt{ }$ & $\sqrt{ }$ & $\sqrt{ }$ & 1.4 & 0.4 & 5 \\
\hline 40 & $\sqrt{ }$ & & & $\sqrt{ }$ & & & & & & $\sqrt{ }$ & & $\sqrt{ }$ & - & - & - \\
\hline 41 & $\sqrt{ }$ & $\sqrt{ }$ & & & & & & & $\sqrt{ }$ & & $\sqrt{ }$ & $\sqrt{ }$ & 0.9 & -3.0 & 7 \\
\hline 42 & $\sqrt{ }$ & $\sqrt{ }$ & & & $\checkmark$ & & $\sqrt{ }$ & & & $\sqrt{ }$ & $\sqrt{ }$ & $\sqrt{ }$ & 2.0 & 0.6 & 9 \\
\hline 43 & $\sqrt{ }$ & $\sqrt{ }$ & & & $\checkmark$ & & $\sqrt{ }$ & & $\sqrt{ }$ & & $\sqrt{ }$ & $\sqrt{ }$ & 2.5 & -6.0 & 4 \\
\hline 44 & $\sqrt{ }$ & $\sqrt{ }$ & $\sqrt{ }$ & $\sqrt{ }$ & & & $\sqrt{ }$ & & $\sqrt{ }$ & $\sqrt{ }$ & $\sqrt{ }$ & $\sqrt{ }$ & 0.5 & 0.3 & 7 \\
\hline 45 & $\sqrt{ }$ & $\sqrt{ }$ & & & $\sqrt{ }$ & & & & & & $\sqrt{ }$ & $\sqrt{ }$ & 1.2 & -1.6 & 5 \\
\hline 46 & $\sqrt{ }$ & & & & $\sqrt{ }$ & $\sqrt{ }$ & & & & $\sqrt{ }$ & $\sqrt{ }$ & $\sqrt{ }$ & -1.8 & 0.9 & 4 \\
\hline 47 & $\sqrt{ }$ & & $\checkmark$ & $\sqrt{ }$ & $\sqrt{ }$ & $\sqrt{ }$ & & & $\sqrt{ }$ & $\sqrt{ }$ & $\sqrt{ }$ & $\sqrt{ }$ & -0.4 & -4.2 & 2 \\
\hline 48 & $\sqrt{ }$ & $V$ & & & & & $\sqrt{ }$ & & & & $\sqrt{ }$ & $\checkmark$ & -2.8 & 2.7 & 13 \\
\hline 49 & $\sqrt{ }$ & $\sqrt{ }$ & $\checkmark$ & $\checkmark$ & & & & $\sqrt{ }$ & $\sqrt{ }$ & $\checkmark$ & $\sqrt{ }$ & $\checkmark$ & -1.9 & -0.5 & 7 \\
\hline 50 & & & $\sqrt{ }$ & & & & & & $\sqrt{ }$ & $\sqrt{ }$ & $\checkmark$ & $\sqrt{ }$ & -1.5 & -1.2 & 9 \\
\hline
\end{tabular}


Table 55: Model's change in VTR prediction for worksites with Transit share between $5 \%$ to $15 \%$ and Vehicle Trip Rate between 60 to 70

\begin{tabular}{|c|c|c|c|c|c|c|c|c|c|c|c|c|c|c|c|}
\hline $\begin{array}{l}\text { Sr. } \\
\text { No. }\end{array}$ & $F \& A$ & ONS & СТВ & FI N & CWW & FLEX & TELE & PMT & NONF & GRH & RSMP & MRKT & $\begin{array}{r}\text { Tran } \\
{\left[0.0^{-1}\right.} \\
\text { VTR } \\
\end{array}$ & $\begin{array}{l}\text { nsit Sha } \\
05-0.15 \\
-[70-60\end{array}$ & \\
\hline & & & & & & & & & & & & & Predicted & Actual & No.Ex \\
\hline 1 & $\sqrt{ }$ & $\sqrt{ }$ & $\checkmark$ & $\checkmark$ & & & & & $\sqrt{ }$ & $\sqrt{ }$ & $\sqrt{ }$ & $\sqrt{ }$ & -0.1 & 2.0 & 16 \\
\hline 2 & $\sqrt{ }$ & $\sqrt{ }$ & $\sqrt{ }$ & $\sqrt{ }$ & & $\sqrt{ }$ & & & $\sqrt{ }$ & $\sqrt{ }$ & $\sqrt{ }$ & $\sqrt{3}$ & 1.7 & 4.2 & 8 \\
\hline 3 & $\sqrt{ }$ & $\sqrt{ }$ & $\sqrt{ }$ & & & & & & $\sqrt{ }$ & $\sqrt{ }$ & $\sqrt{ }$ & $\sqrt{ }$ & 1.5 & -0.5 & 15 \\
\hline 4 & $\sqrt{ }$ & & $\sqrt{ }$ & $\sqrt{ }$ & & & & & $\sqrt{ }$ & $\sqrt{ }$ & $\sqrt{ }$ & $\sqrt{ }$ & 0.3 & 5.5 & 8 \\
\hline 5 & $\sqrt{ }$ & $\sqrt{ }$ & & & & & & & $\sqrt{ }$ & $\sqrt{ }$ & $\sqrt{ }$ & $\sqrt{ }$ & 1.9 & 3.4 & 14 \\
\hline 6 & & $\sqrt{ }$ & $\sqrt{ }$ & $\checkmark$ & & & & & $\sqrt{ }$ & $\checkmark$ & $\sqrt{ }$ & $\sqrt{ }$ & 0.5 & 3.0 & 10 \\
\hline 7 & $\sqrt{ }$ & $\sqrt{ }$ & & & & $\sqrt{ }$ & & & $\sqrt{ }$ & $\sqrt{ }$ & $\sqrt{ }$ & $\sqrt{ }$ & 2.9 & 7.2 & 12 \\
\hline 8 & $\sqrt{ }$ & $\sqrt{ }$ & $\checkmark$ & $\sqrt{ }$ & $\sqrt{ }$ & & & & $\sqrt{ }$ & $\sqrt{ }$ & $\sqrt{ }$ & $\sqrt{ }$ & -1.1 & 5.7 & 4 \\
\hline 9 & $\sqrt{ }$ & & $\sqrt{ }$ & & & & & & $\sqrt{ }$ & $\sqrt{ }$ & $\sqrt{ }$ & $\sqrt{ }$ & 0.4 & 2.1 & 11 \\
\hline 10 & $\sqrt{ }$ & $\sqrt{ }$ & & & $\sqrt{ }$ & & & & $\sqrt{ }$ & $\sqrt{ }$ & $\sqrt{ }$ & $\sqrt{ }$ & 1.1 & 0.7 & 7 \\
\hline 11 & $\sqrt{ }$ & & $\sqrt{ }$ & $\sqrt{ }$ & & & & & & $\sqrt{ }$ & $\sqrt{ }$ & $\sqrt{ }$ & 0.3 & 5.5 & 8 \\
\hline 12 & $\sqrt{ }$ & & & & & & & & & $\sqrt{ }$ & $\sqrt{ }$ & $\sqrt{ }$ & 1.8 & 1.9 & 16 \\
\hline 13 & $\checkmark$ & $\sqrt{ }$ & $\checkmark$ & & & $\sqrt{ }$ & & & $\sqrt{ }$ & $\sqrt{ }$ & $\checkmark$ & $\checkmark$ & 1.1 & 1.8 & 9 \\
\hline 14 & $\sqrt{ }$ & $\sqrt{ }$ & $\sqrt{ }$ & $\sqrt{ }$ & & & & & & $\sqrt{ }$ & $\sqrt{ }$ & $\sqrt{ }$ & 2.1 & 5.7 & 6 \\
\hline 15 & $\sqrt{ }$ & & & & & & & & $\sqrt{ }$ & $\sqrt{ }$ & $\sqrt{ }$ & $\sqrt{ }$ & 1.7 & 2.1 & 10 \\
\hline 16 & $\sqrt{ }$ & $\sqrt{ }$ & $\sqrt{ }$ & $\checkmark$ & $\sqrt{ }$ & $\sqrt{ }$ & & & $\sqrt{ }$ & $\sqrt{ }$ & $\sqrt{ }$ & $\checkmark$ & 3.7 & -3.3 & 4 \\
\hline 17 & $\sqrt{ }$ & $\sqrt{ }$ & $\checkmark$ & $\checkmark$ & & & & & $\sqrt{ }$ & $\sqrt{ }$ & & $\checkmark$ & -1.0 & 2.0 & 8 \\
\hline 18 & $\sqrt{ }$ & $\sqrt{ }$ & $\sqrt{ }$ & & $\sqrt{ }$ & & & & $\sqrt{ }$ & $\sqrt{ }$ & $\sqrt{ }$ & $\sqrt{ }$ & 2.7 & 5.3 & 4 \\
\hline 19 & $\sqrt{ }$ & & $\sqrt{ }$ & $\sqrt{ }$ & & $\sqrt{ }$ & & & $\sqrt{ }$ & $\sqrt{ }$ & $\sqrt{ }$ & $\sqrt{ }$ & 0.7 & 4.1 & 5 \\
\hline 20 & & & $\sqrt{ }$ & $\sqrt{ }$ & & & & & $\sqrt{ }$ & $\sqrt{ }$ & $\sqrt{ }$ & $\sqrt{ }$ & -1.3 & 5.3 & 5 \\
\hline 21 & $\sqrt{ }$ & $\sqrt{ }$ & & & $\checkmark$ & & $\sqrt{ }$ & & $\sqrt{ }$ & $\checkmark$ & $\checkmark$ & $\sqrt{ }$ & 2.1 & 0.6 & 8 \\
\hline 22 & $\sqrt{ }$ & & $\checkmark$ & & & $\sqrt{ }$ & & & $\sqrt{ }$ & $\sqrt{ }$ & $\sqrt{ }$ & $\sqrt{ }$ & -0.9 & 4.7 & 11 \\
\hline 23 & $\sqrt{ }$ & & & & & & & & & & $\sqrt{ }$ & $\checkmark$ & 1.5 & 2.2 & 7 \\
\hline 24 & $\sqrt{ }$ & $\sqrt{ }$ & & $\sqrt{ }$ & & & & & $\sqrt{ }$ & $\sqrt{ }$ & $\sqrt{ }$ & $\checkmark$ & 0.4 & -3.3 & 2 \\
\hline 25 & $\sqrt{ }$ & $\sqrt{ }$ & $\sqrt{ }$ & & $\sqrt{ }$ & $\sqrt{ }$ & & & $\sqrt{ }$ & $\sqrt{ }$ & $\sqrt{ }$ & $\sqrt{ }$ & 3.1 & 3.8 & 1 \\
\hline 26 & $\sqrt{ }$ & $\sqrt{ }$ & $\sqrt{ }$ & $\sqrt{ }$ & & $\sqrt{ }$ & & & & $\sqrt{ }$ & $\sqrt{ }$ & $\checkmark$ & 2.6 & -6.6 & 2 \\
\hline 27 & $\sqrt{ }$ & $\sqrt{ }$ & & & & & & & & & $\sqrt{ }$ & $\sqrt{ }$ & 2.2 & 2.4 & 4 \\
\hline 28 & $\sqrt{ }$ & $\sqrt{ }$ & $\checkmark$ & & $\checkmark$ & & $\sqrt{ }$ & & $\sqrt{ }$ & $\sqrt{ }$ & $\sqrt{ }$ & $\sqrt{ }$ & 2.7 & 2.9 & 2 \\
\hline 29 & $\sqrt{ }$ & $\sqrt{ }$ & $\checkmark$ & & & & & & $\sqrt{ }$ & $\sqrt{ }$ & & $\sqrt{ }$ & 2.3 & 0.0 & 5 \\
\hline 30 & $\sqrt{ }$ & & $\sqrt{ }$ & $\sqrt{ }$ & $\sqrt{ }$ & & & & $\sqrt{ }$ & $\sqrt{ }$ & $\sqrt{ }$ & $\sqrt{ }$ & -1.3 & -3.4 & 1 \\
\hline 31 & $\sqrt{ }$ & $\sqrt{ }$ & - & & & & & & & $\sqrt{ }$ & $\sqrt{ }$ & $\sqrt{ }$ & 1.8 & -0.7 & 10 \\
\hline 32 & $\sqrt{ }$ & & & & & $\sqrt{ }$ & & & $\sqrt{ }$ & $\sqrt{ }$ & $\sqrt{ }$ & $\checkmark$ & 1.9 & 8.2 & 3 \\
\hline 33 & & $\sqrt{ }$ & $\checkmark$ & & & & & & $\sqrt{ }$ & $\sqrt{ }$ & $\sqrt{ }$ & $\checkmark$ & 0.6 & 12.2 & 6 \\
\hline 34 & $\checkmark$ & $\sqrt{ }$ & & $\sqrt{ }$ & $\sqrt{ }$ & $\sqrt{ }$ & & & $\sqrt{ }$ & $\sqrt{ }$ & $\checkmark$ & $\sqrt{ }$ & 2.5 & 3.4 & 1 \\
\hline 35 & $\sqrt{ }$ & $\sqrt{ }$ & $\sqrt{ }$ & & & & & & & $\sqrt{ }$ & $\sqrt{ }$ & $\sqrt{ }$ & 1.6 & 0.6 & 4 \\
\hline 36 & $\sqrt{ }$ & & $\sqrt{ }$ & $\checkmark$ & & & & & $\sqrt{ }$ & $\sqrt{ }$ & & $\sqrt{ }$ & -1.1 & 1.5 & 2 \\
\hline 37 & $\sqrt{ }$ & & & $\sqrt{ }$ & & & & & $\sqrt{ }$ & $\sqrt{ }$ & $\sqrt{ }$ & $\sqrt{ }$ & - & - & - \\
\hline 38 & $\checkmark$ & $\sqrt{ }$ & $\checkmark$ & $\checkmark$ & $\checkmark$ & & $\checkmark$ & & $\sqrt{ }$ & $\sqrt{ }$ & $\checkmark$ & $\checkmark$ & - & - & - \\
\hline 39 & $\sqrt{ }$ & $\sqrt{ }$ & $\checkmark$ & $\checkmark$ & $\checkmark$ & $\sqrt{ }$ & $\sqrt{ }$ & & $\sqrt{ }$ & $\sqrt{ }$ & $\sqrt{ }$ & $\sqrt{ }$ & 3.6 & 13.1 & 1 \\
\hline 40 & $\sqrt{ }$ & & & $\sqrt{ }$ & & & & & & $\sqrt{ }$ & & $\sqrt{ }$ & 0.9 & 11.4 & 1 \\
\hline 41 & $\sqrt{ }$ & $\sqrt{ }$ & & & & & & & $\sqrt{ }$ & & $\sqrt{ }$ & $\sqrt{ }$ & 3.1 & 3.8 & 8 \\
\hline 42 & $\sqrt{ }$ & $\sqrt{ }$ & & & $\sqrt{ }$ & & $\sqrt{ }$ & & & $\sqrt{ }$ & $\sqrt{ }$ & $\checkmark$ & 0.8 & -2.3 & 5 \\
\hline 43 & $\sqrt{ }$ & $\sqrt{ }$ & & & $\checkmark$ & & $\sqrt{ }$ & & $\sqrt{ }$ & & $\sqrt{ }$ & $\checkmark$ & - & - & - \\
\hline 44 & $\sqrt{ }$ & $\sqrt{ }$ & $\sqrt{ }$ & $\sqrt{ }$ & & & $\sqrt{ }$ & & $\sqrt{ }$ & $\sqrt{ }$ & $\sqrt{ }$ & $\sqrt{ }$ & - & - & - \\
\hline 45 & $\sqrt{ }$ & $\sqrt{ }$ & & & $\sqrt{ }$ & & & & & & $\sqrt{ }$ & $\sqrt{ }$ & 0.0 & 17.3 & 1 \\
\hline 46 & $\sqrt{ }$ & & & & $\sqrt{ }$ & $\sqrt{ }$ & & & & $\sqrt{ }$ & $\sqrt{ }$ & $\sqrt{ }$ & 1.1 & 3.5 & 1 \\
\hline 47 & $\sqrt{ }$ & & $\checkmark$ & $\sqrt{ }$ & $\sqrt{ }$ & $\sqrt{ }$ & & & $\sqrt{ }$ & $\sqrt{ }$ & $\sqrt{ }$ & $\sqrt{ }$ & -2.2 & -1.1 & 1 \\
\hline 48 & $\sqrt{ }$ & $V$ & & & & & $\sqrt{ }$ & & & & $\sqrt{ }$ & $\checkmark$ & -1.7 & 13.1 & 5 \\
\hline 49 & $\sqrt{ }$ & $\sqrt{ }$ & $\checkmark$ & $\checkmark$ & & & & $\sqrt{ }$ & $\sqrt{ }$ & $\checkmark$ & $\sqrt{ }$ & $\checkmark$ & 1.7 & -2.3 & 5 \\
\hline 50 & & & $\sqrt{ }$ & & & & & & $\sqrt{ }$ & $\sqrt{ }$ & $\checkmark$ & $\sqrt{ }$ & 0.3 & -0.4 & 10 \\
\hline
\end{tabular}


Table 56: Model's change in VTR prediction for worksites with Transit share between $5 \%$ to $15 \%$ and Vehicle Trip Rate between 50 to 60

\begin{tabular}{|c|c|c|c|c|c|c|c|c|c|c|c|c|c|c|c|}
\hline $\begin{array}{l}\text { Sr. } \\
\text { No. }\end{array}$ & $F \& A$ & ONS & СТВ & FI N & CWW & FLEX & TELE & PMT & NONF & GRH & RSMP & MRKT & $\begin{array}{r}\text { Tran } \\
{\left[0.0^{-1}\right.} \\
\text { VTR } \\
\end{array}$ & $\begin{array}{l}\text { nsit Sha } \\
05-0.15 \\
-[60-50\end{array}$ & \\
\hline & & & & & & & & & & & & & Predicted & Actual & No.Ex \\
\hline 1 & $\sqrt{ }$ & $\sqrt{ }$ & $\checkmark$ & $\checkmark$ & & & & & $\sqrt{ }$ & $\sqrt{ }$ & $\sqrt{ }$ & $\sqrt{ }$ & 3.7 & 1.4 & 1 \\
\hline 2 & $\sqrt{ }$ & $\sqrt{ }$ & $\sqrt{ }$ & $\sqrt{ }$ & & $\sqrt{ }$ & & & $\sqrt{ }$ & $\sqrt{ }$ & $\sqrt{ }$ & $\sqrt{ }$ & 0.4 & 0.7 & 1 \\
\hline 3 & $\sqrt{ }$ & $\sqrt{ }$ & $\sqrt{ }$ & & & & & & $\sqrt{ }$ & $\sqrt{ }$ & $\sqrt{ }$ & $\sqrt{ }$ & 2.4 & 5.3 & 2 \\
\hline 4 & $\sqrt{ }$ & & $\sqrt{ }$ & $\sqrt{ }$ & & & & & $\sqrt{ }$ & $\sqrt{ }$ & $\sqrt{ }$ & $\sqrt{ }$ & - & - & - \\
\hline 5 & $\sqrt{ }$ & $\sqrt{ }$ & & & & & & & $\sqrt{ }$ & $\sqrt{ }$ & $\sqrt{ }$ & $\sqrt{ }$ & 0.7 & 6.8 & 3 \\
\hline 6 & & $\sqrt{ }$ & $\sqrt{ }$ & $\checkmark$ & & & & & $\sqrt{ }$ & $\checkmark$ & $\sqrt{ }$ & $\sqrt{ }$ & - & - & - \\
\hline 7 & $\sqrt{ }$ & $\sqrt{ }$ & & & & $\sqrt{ }$ & & & $\sqrt{ }$ & $\sqrt{ }$ & $\sqrt{ }$ & $\sqrt{ }$ & 6.3 & 7.4 & 1 \\
\hline 8 & $\sqrt{ }$ & $\sqrt{ }$ & $\sqrt{ }$ & $\sqrt{ }$ & $\sqrt{ }$ & & & & $\sqrt{ }$ & $\sqrt{ }$ & $\sqrt{ }$ & $\sqrt{ }$ & - & - & - \\
\hline 9 & $\sqrt{ }$ & & $\sqrt{ }$ & & & & & & $\sqrt{ }$ & $\sqrt{ }$ & $\sqrt{ }$ & $\sqrt{ }$ & -1.0 & 2.3 & 3 \\
\hline 10 & $\sqrt{ }$ & $\sqrt{ }$ & & & $\sqrt{ }$ & & & & $\sqrt{ }$ & $\sqrt{ }$ & $\sqrt{ }$ & $\sqrt{ }$ & 6.0 & 12.0 & 1 \\
\hline 11 & $\sqrt{ }$ & & $\sqrt{ }$ & $\sqrt{ }$ & & & & & & $\sqrt{ }$ & $\sqrt{ }$ & $\sqrt{ }$ & - & - & - \\
\hline 12 & $\sqrt{ }$ & & & & & & & & & $\sqrt{ }$ & $\sqrt{ }$ & $\sqrt{ }$ & 4.1 & 5.0 & 4 \\
\hline 13 & $\checkmark$ & $\sqrt{ }$ & $\checkmark$ & & & $\sqrt{ }$ & & & $\sqrt{ }$ & $\sqrt{ }$ & $\checkmark$ & $\checkmark$ & $=$ & - & - \\
\hline 14 & $\sqrt{ }$ & $\sqrt{ }$ & $\sqrt{ }$ & $\sqrt{ }$ & & & & & & $\sqrt{ }$ & $\sqrt{ }$ & $\sqrt{ }$ & 1.3 & 2.0 & 3 \\
\hline 15 & $\sqrt{ }$ & & & & & & & & $\sqrt{ }$ & $\sqrt{ }$ & $\sqrt{ }$ & $\sqrt{ }$ & 5.2 & 4.7 & 2 \\
\hline 16 & $\sqrt{ }$ & $\sqrt{ }$ & $\sqrt{ }$ & $\sqrt{ }$ & $\sqrt{ }$ & $\sqrt{ }$ & & & $\sqrt{ }$ & $\sqrt{ }$ & $\sqrt{ }$ & $\sqrt{ }$ & - & - & - \\
\hline 17 & $\sqrt{ }$ & $\sqrt{ }$ & $\sqrt{ }$ & $\sqrt{ }$ & & & & & $\sqrt{ }$ & $\checkmark$ & & $\checkmark$ & - & - & - \\
\hline 18 & $\sqrt{ }$ & $\sqrt{ }$ & $\sqrt{ }$ & . & $\sqrt{ }$ & & & & $\sqrt{ }$ & $\checkmark$ & $\sqrt{ }$ & $\sqrt{ }$ & 4.4 & 9.9 & 2 \\
\hline 19 & $\sqrt{ }$ & & $\sqrt{ }$ & $\sqrt{ }$ & & $\sqrt{ }$ & & & $\sqrt{ }$ & $\sqrt{ }$ & $\sqrt{ }$ & $\sqrt{ }$ & - & - & - \\
\hline 20 & & & $\sqrt{ }$ & $\sqrt{ }$ & & & & & $\sqrt{ }$ & $\sqrt{ }$ & $\sqrt{ }$ & $\sqrt{ }$ & 1.3 & 6.9 & 1 \\
\hline 21 & $\sqrt{ }$ & $\sqrt{ }$ & & & $\sqrt{ }$ & & $\sqrt{ }$ & & $\sqrt{ }$ & $\sqrt{ }$ & $\sqrt{ }$ & $\sqrt{ }$ & - & - & - \\
\hline 22 & $\sqrt{ }$ & & $\sqrt{ }$ & & & $\sqrt{ }$ & & & $\sqrt{ }$ & $\sqrt{ }$ & $\sqrt{ }$ & $\sqrt{ }$ & 1.0 & 7.1 & 4 \\
\hline 23 & $\sqrt{ }$ & & & & & & & & & & $\sqrt{ }$ & $\sqrt{ }$ & 4.5 & 2.0 & 3 \\
\hline 24 & $\sqrt{ }$ & $\sqrt{ }$ & 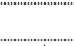 & $\sqrt{ }$ & & . & & & $\sqrt{ }$ & $\sqrt{ }$ & $\sqrt{ }$ & $\checkmark$ & - & - & - \\
\hline 25 & $\sqrt{ }$ & $\sqrt{ }$ & $\sqrt{ }$ & & $\sqrt{ }$ & $\sqrt{ }$ & & & $\sqrt{ }$ & $\sqrt{ }$ & $\sqrt{ }$ & $\sqrt{ }$ & - & - & - \\
\hline 26 & $\sqrt{ }$ & $\sqrt{ }$ & $\sqrt{ }$ & $\sqrt{ }$ & & $\sqrt{ }$ & & & & $\sqrt{ }$ & $\sqrt{ }$ & $\checkmark$ & 1.1 & 5.0 & 1 \\
\hline 27 & $\sqrt{ }$ & $\sqrt{ }$ & & & & & & & & & $\sqrt{ }$ & $\sqrt{ }$ & - & - & - \\
\hline 28 & $\sqrt{ }$ & $\sqrt{ }$ & $\sqrt{ }$ & & $\sqrt{ }$ & & $\sqrt{ }$ & & $\sqrt{ }$ & $\sqrt{ }$ & $\sqrt{ }$ & $\sqrt{ }$ & - & - & - \\
\hline 29 & $\sqrt{ }$ & $\sqrt{ }$ & $\checkmark$ & & & & & & $\sqrt{ }$ & $\sqrt{ }$ & & $\sqrt{ }$ & 0.4 & 6.4 & 3 \\
\hline 30 & $\sqrt{ }$ & & $\sqrt{ }$ & $\sqrt{ }$ & $\sqrt{ }$ & & & & $\sqrt{ }$ & $\sqrt{ }$ & $\sqrt{ }$ & $\sqrt{ }$ & 3.0 & 2.1 & 2 \\
\hline 31 & $\sqrt{ }$ & $\sqrt{ }$ & - & & & & & & & $\sqrt{ }$ & $\sqrt{ }$ & $\sqrt{ }$ & 4.3 & -1.8 & 3 \\
\hline 32 & $\sqrt{ }$ & & & & & $\sqrt{ }$ & & & $\sqrt{ }$ & $\sqrt{ }$ & $\sqrt{ }$ & $\checkmark$ & 3.3 & 5.1 & 3 \\
\hline 33 & & $\sqrt{ }$ & $\checkmark$ & & & & & & $\sqrt{ }$ & $\sqrt{ }$ & $\sqrt{ }$ & $\checkmark$ & 4.1 & -0.6 & 3 \\
\hline 34 & $\checkmark$ & $\sqrt{ }$ & & $\sqrt{ }$ & $\sqrt{ }$ & $\sqrt{ }$ & & & $\sqrt{ }$ & $\sqrt{ }$ & $\checkmark$ & $\sqrt{ }$ & $=$ & - & - \\
\hline 35 & $\sqrt{ }$ & $\sqrt{ }$ & $\sqrt{ }$ & & & & & & & $\sqrt{ }$ & $\sqrt{ }$ & $\sqrt{ }$ & 4.8 & 4.6 & 4 \\
\hline 36 & $\sqrt{ }$ & & $\sqrt{ }$ & $\sqrt{ }$ & & & & & $\sqrt{ }$ & $\sqrt{ }$ & & $\sqrt{ }$ & 0.5 & -5.2 & 2 \\
\hline 37 & $\sqrt{ }$ & & & $\sqrt{ }$ & & & & & $\sqrt{ }$ & $\sqrt{ }$ & $\sqrt{ }$ & $\sqrt{ }$ & 3.8 & 12.9 & 1 \\
\hline 38 & $\checkmark$ & $\sqrt{ }$ & $\checkmark$ & $\checkmark$ & $\checkmark$ & & $\checkmark$ & & $\sqrt{ }$ & $\sqrt{ }$ & $\checkmark$ & $\checkmark$ & - & - & - \\
\hline 39 & $\sqrt{ }$ & $\sqrt{ }$ & $\sqrt{ }$ & $\checkmark$ & $\checkmark$ & $\sqrt{ }$ & $\sqrt{ }$ & & $\sqrt{ }$ & $\checkmark$ & $\sqrt{ }$ & $\sqrt{ }$ & - & - & - \\
\hline 40 & $\sqrt{ }$ & & & $\sqrt{ }$ & & & & & & $\sqrt{ }$ & & $\sqrt{ }$ & - & - & - \\
\hline 41 & $\sqrt{ }$ & $\sqrt{ }$ & & & & & & & $\sqrt{ }$ & & $\sqrt{ }$ & $\sqrt{ }$ & 4.6 & 4.2 & 2 \\
\hline 42 & $\sqrt{ }$ & $\sqrt{ }$ & & & $\checkmark$ & & $\sqrt{ }$ & & & $\sqrt{ }$ & $\sqrt{ }$ & $\sqrt{ }$ & - & - & - \\
\hline 43 & $\sqrt{ }$ & $\sqrt{ }$ & & & $\sqrt{ }$ & & $\sqrt{ }$ & & $\sqrt{ }$ & & $\sqrt{ }$ & $\sqrt{ }$ & - & - & - \\
\hline 44 & $\sqrt{ }$ & $\sqrt{ }$ & $\sqrt{ }$ & $\sqrt{ }$ & & & $\sqrt{ }$ & & $\sqrt{ }$ & $\sqrt{ }$ & $\sqrt{ }$ & $\sqrt{ }$ & - & - & - \\
\hline 45 & $\sqrt{ }$ & $\sqrt{ }$ & & & $\sqrt{ }$ & & & & & & $\sqrt{ }$ & $\sqrt{ }$ & - & - & - \\
\hline 46 & $\sqrt{ }$ & & & & $\sqrt{ }$ & $\sqrt{ }$ & & & & $\sqrt{ }$ & $\sqrt{ }$ & $\sqrt{ }$ & - & - & - \\
\hline 47 & $\sqrt{ }$ & & $\checkmark$ & $\sqrt{ }$ & $\sqrt{ }$ & $\sqrt{ }$ & & & $\sqrt{ }$ & $\sqrt{ }$ & $\sqrt{ }$ & $\sqrt{ }$ & $=$ & - & - \\
\hline 48 & $\sqrt{ }$ & $V$ & & & & & $\sqrt{ }$ & & & & $\sqrt{ }$ & $\checkmark$ & -1.6 & 17.3 & 2 \\
\hline 49 & $\sqrt{ }$ & $\sqrt{ }$ & $\checkmark$ & $\checkmark$ & & & & $\sqrt{ }$ & $\sqrt{ }$ & $\checkmark$ & $\sqrt{ }$ & $\checkmark$ & 5.2 & 2.7 & 1 \\
\hline 50 & & & $\sqrt{ }$ & & & & & & $\sqrt{ }$ & $\sqrt{ }$ & $\checkmark$ & $\sqrt{ }$ & 1.9 & -2.7 & 1 \\
\hline
\end{tabular}


Table 57: Model's change in VTR prediction for worksites with Transit share between $15 \%$ to $25 \%$ and Vehicle Trip Rate between 70 to 80

\begin{tabular}{|c|c|c|c|c|c|c|c|c|c|c|c|c|c|c|c|}
\hline $\begin{array}{l}\text { Sr. } \\
\text { No. }\end{array}$ & $F \& A$ & ONS & СТВ & FI N & CWW & FLEX & TELE & PMT & NONF & GRH & RSMP & MRKT & $\begin{array}{r}\text { Tran } \\
{[0 .]} \\
\text { VTR } \\
\end{array}$ & $\begin{array}{l}\text { nsit Sha } \\
15-0.25 \\
-[80-70\end{array}$ & \\
\hline & & & & & & & & & & & & & Predicted & Actual & No.Ex \\
\hline 1 & $\sqrt{ }$ & $\sqrt{ }$ & $\checkmark$ & $\checkmark$ & & & & & $\sqrt{ }$ & $\sqrt{ }$ & $\sqrt{ }$ & $\sqrt{ }$ & -3.2 & -5.0 & 5 \\
\hline 2 & $\sqrt{ }$ & $\sqrt{ }$ & $\sqrt{ }$ & $\sqrt{ }$ & & $\sqrt{ }$ & & & $\sqrt{ }$ & $\sqrt{ }$ & $\sqrt{ }$ & $\sqrt{3}$ & -1.9 & -4.4 & 4 \\
\hline 3 & $\sqrt{ }$ & $\sqrt{ }$ & $\sqrt{ }$ & & & & & & $\sqrt{ }$ & $\sqrt{ }$ & $\sqrt{ }$ & $\sqrt{ }$ & -3.5 & -4.5 & 7 \\
\hline 4 & $\sqrt{ }$ & & $\sqrt{ }$ & $\sqrt{ }$ & & & & & $\sqrt{ }$ & $\sqrt{ }$ & $\sqrt{ }$ & $\sqrt{ }$ & -3.3 & 6.1 & 2 \\
\hline 5 & $\sqrt{ }$ & $\sqrt{ }$ & & & & & & & $\sqrt{ }$ & $\sqrt{ }$ & $\sqrt{ }$ & $\sqrt{ }$ & -1.6 & 1.1 & 5 \\
\hline 6 & & $\sqrt{ }$ & $\sqrt{ }$ & $\checkmark$ & & & & & $\sqrt{ }$ & $\checkmark$ & $\sqrt{ }$ & $\sqrt{ }$ & -3.5 & 0.7 & 8 \\
\hline 7 & $\sqrt{ }$ & $\sqrt{ }$ & & & & $\sqrt{ }$ & & & $\sqrt{ }$ & $\sqrt{ }$ & $\sqrt{ }$ & $\sqrt{n}$ & -2.4 & -10.7 & 1 \\
\hline 8 & $\sqrt{ }$ & $\sqrt{ }$ & $\checkmark$ & $\sqrt{ }$ & $\sqrt{ }$ & & & & $\sqrt{ }$ & $\sqrt{ }$ & $\sqrt{ }$ & $\sqrt{ }$ & - & - & - \\
\hline 9 & $\sqrt{ }$ & & $\sqrt{ }$ & & & & & & $\sqrt{ }$ & $\sqrt{ }$ & $\sqrt{ }$ & $\sqrt{ }$ & -6.3 & -6.2 & 1 \\
\hline 10 & $\sqrt{ }$ & $\sqrt{ }$ & & & $\sqrt{ }$ & & & & $\sqrt{ }$ & $\sqrt{ }$ & $\sqrt{ }$ & $\sqrt{ }$ & -1.0 & 2.2 & 1 \\
\hline 11 & $\sqrt{ }$ & & $\sqrt{ }$ & $\sqrt{ }$ & & & & & & $\sqrt{ }$ & $\sqrt{ }$ & $\sqrt{ }$ & -3.3 & 6.1 & 2 \\
\hline 12 & $\sqrt{ }$ & & & & & & & & & $\sqrt{ }$ & $\sqrt{ }$ & $\sqrt{ }$ & - & - & - \\
\hline 13 & $\checkmark$ & $\sqrt{ }$ & $\checkmark$ & & & $\sqrt{ }$ & & & $\sqrt{ }$ & $\sqrt{ }$ & $\checkmark$ & $\checkmark$ & -2.0 & -5.5 & 1 \\
\hline 14 & $\sqrt{ }$ & $\sqrt{ }$ & $\sqrt{ }$ & $\sqrt{ }$ & & & & & & $\sqrt{ }$ & $\sqrt{ }$ & $\checkmark$ & -5.6 & 4.9 & 1 \\
\hline 15 & $\sqrt{ }$ & & & & & & & & $\sqrt{ }$ & $\sqrt{ }$ & $\sqrt{ }$ & $\sqrt{ }$ & -1.7 & -2.2 & 1 \\
\hline 16 & $\sqrt{ }$ & $\sqrt{ }$ & $\sqrt{ }$ & $\sqrt{ }$ & $\sqrt{ }$ & $\sqrt{ }$ & & & $\sqrt{ }$ & $\sqrt{ }$ & $\sqrt{ }$ & $\sqrt{ }$ & - & - & - \\
\hline 17 & $\sqrt{ }$ & $\sqrt{ }$ & $\sqrt{ }$ & $\sqrt{ }$ & & & & & $\sqrt{ }$ & $\checkmark$ & & $\checkmark$ & -2.7 & -1.9 & 3 \\
\hline 18 & $\sqrt{ }$ & $\sqrt{ }$ & $\sqrt{ }$ & 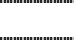 & $\sqrt{ }$ & & & & $\sqrt{ }$ & $\sqrt{ }$ & $\sqrt{ }$ & $\sqrt{ }$ & - & - & - \\
\hline 19 & $\sqrt{ }$ & & $\sqrt{ }$ & $\sqrt{ }$ & & $\sqrt{ }$ & & & $\sqrt{ }$ & $\sqrt{ }$ & $\sqrt{ }$ & $\sqrt{ }$ & - & - & - \\
\hline 20 & & & $\sqrt{ }$ & $\sqrt{ }$ & & & & & $\sqrt{ }$ & $\sqrt{ }$ & $\sqrt{ }$ & $\sqrt{ }$ & -3.7 & -0.8 & 3 \\
\hline 21 & $\sqrt{ }$ & $\sqrt{ }$ & & & $\sqrt{ }$ & & $\sqrt{ }$ & & $\sqrt{ }$ & $\sqrt{ }$ & $\sqrt{ }$ & $\sqrt{ }$ & - & - & - \\
\hline 22 & $\sqrt{ }$ & & $\sqrt{ }$ & & & $\sqrt{ }$ & & & $\sqrt{ }$ & $\sqrt{ }$ & $\sqrt{ }$ & $\sqrt{ }$ & - & - & - \\
\hline 23 & $\sqrt{ }$ & & & & & & & & & & $\sqrt{ }$ & $\sqrt{ }$ & - & - & - \\
\hline 24 & $\sqrt{ }$ & $\sqrt{ }$ & 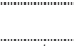 & $\sqrt{ }$ & & 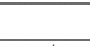 & & & $\sqrt{ }$ & $\sqrt{ }$ & $\sqrt{ }$ & $\checkmark$ & - & - & - \\
\hline 25 & $\sqrt{ }$ & $\sqrt{ }$ & $\sqrt{ }$ & & $\sqrt{ }$ & $\sqrt{ }$ & & & $\sqrt{ }$ & $\sqrt{ }$ & $\sqrt{ }$ & $\sqrt{ }$ & -0.7 & 6.4 & 1 \\
\hline 26 & $\sqrt{ }$ & $\sqrt{ }$ & $\sqrt{ }$ & $\sqrt{ }$ & & $\sqrt{ }$ & & & & $\sqrt{ }$ & $\sqrt{ }$ & $\checkmark$ & -1.6 & 9.5 & 1 \\
\hline 27 & $\sqrt{ }$ & $\sqrt{ }$ & & & & & & & & & $\sqrt{ }$ & $\sqrt{ }$ & 0.7 & 4.4 & 2 \\
\hline 28 & $\sqrt{ }$ & $\sqrt{ }$ & $\sqrt{ }$ & & $\sqrt{ }$ & & $\sqrt{ }$ & & $\sqrt{ }$ & $\sqrt{ }$ & $\sqrt{ }$ & $\sqrt{ }$ & - & - & - \\
\hline 29 & $\sqrt{ }$ & $\sqrt{ }$ & $\checkmark$ & & & & & & $\sqrt{ }$ & $\sqrt{ }$ & & $\sqrt{ }$ & -0.8 & -8.7 & 2 \\
\hline 30 & $\sqrt{ }$ & & $\sqrt{ }$ & $\sqrt{ }$ & $\sqrt{ }$ & & & & $\sqrt{ }$ & $\sqrt{ }$ & $\sqrt{ }$ & $\sqrt{ }$ & -0.9 & 4.7 & 1 \\
\hline 31 & $\sqrt{ }$ & $\sqrt{ }$ & $-\cdots$ & & & & & & & $\sqrt{ }$ & $\sqrt{ }$ & $\sqrt{ }$ & -1.1 & -3.7 & 1 \\
\hline 32 & $\sqrt{ }$ & & & & & $\sqrt{ }$ & & & $\sqrt{ }$ & $\sqrt{ }$ & $\sqrt{ }$ & $\checkmark$ & -3.6 & -11.5 & 3 \\
\hline 33 & & $\sqrt{ }$ & $\checkmark$ & & & & & & $\sqrt{ }$ & $\sqrt{ }$ & $\sqrt{ }$ & $\checkmark$ & -5.1 & -3.0 & 2 \\
\hline 34 & $\checkmark$ & $\sqrt{ }$ & & $\checkmark$ & $\sqrt{ }$ & $\sqrt{ }$ & & & $\sqrt{ }$ & $\sqrt{ }$ & $\checkmark$ & $\sqrt{ }$ & $=$ & - & - \\
\hline 35 & $\sqrt{ }$ & $\sqrt{ }$ & $\sqrt{ }$ & & & & & & & $\sqrt{ }$ & $\sqrt{ }$ & $\sqrt{ }$ & -1.0 & 2.0 & 1 \\
\hline 36 & $\sqrt{ }$ & & $\sqrt{ }$ & $\checkmark$ & & & & & $\sqrt{ }$ & $\sqrt{ }$ & & $\sqrt{ }$ & - & - & - \\
\hline 37 & $\sqrt{ }$ & & & $\sqrt{ }$ & & & & & $\sqrt{ }$ & $\sqrt{ }$ & $\sqrt{ }$ & $\sqrt{ }$ & - & - & - \\
\hline 38 & $\checkmark$ & $\sqrt{ }$ & $\checkmark$ & $\checkmark$ & $\checkmark$ & & $\checkmark$ & & $\sqrt{ }$ & $\sqrt{ }$ & $\checkmark$ & $\checkmark$ & $=$ & - & - \\
\hline 39 & $\sqrt{ }$ & $\sqrt{ }$ & $\sqrt{ }$ & $\checkmark$ & $\checkmark$ & $\sqrt{ }$ & $\sqrt{ }$ & & $\sqrt{ }$ & $\checkmark$ & $\sqrt{ }$ & $\sqrt{ }$ & - & - & - \\
\hline 40 & $\sqrt{ }$ & & & $\sqrt{ }$ & & & & & & $\sqrt{ }$ & & $\sqrt{ }$ & - & - & - \\
\hline 41 & $\sqrt{ }$ & $\sqrt{ }$ & & & & & & & $\sqrt{ }$ & & $\sqrt{ }$ & $\sqrt{ }$ & 3.0 & -3.0 & 1 \\
\hline 42 & $\sqrt{ }$ & $\sqrt{ }$ & & & $\checkmark$ & & $\sqrt{ }$ & & & $\sqrt{ }$ & $\sqrt{ }$ & $\sqrt{ }$ & $=$ & - & - \\
\hline 43 & $\sqrt{ }$ & $\sqrt{ }$ & & & $\sqrt{ }$ & & $\sqrt{ }$ & & $\sqrt{ }$ & & $\sqrt{ }$ & $\sqrt{ }$ & 2.5 & 5.9 & 2 \\
\hline 44 & $\sqrt{ }$ & $\sqrt{ }$ & $\sqrt{ }$ & $\sqrt{ }$ & & & $\sqrt{ }$ & & $\sqrt{ }$ & $\sqrt{ }$ & $\sqrt{ }$ & $\sqrt{ }$ & - & - & - \\
\hline 45 & $\sqrt{ }$ & $\sqrt{ }$ & & & $\sqrt{ }$ & & & & & & $\sqrt{ }$ & $\sqrt{ }$ & - & - & - \\
\hline 46 & $\sqrt{ }$ & & & & $\sqrt{ }$ & $\sqrt{ }$ & & & & $\sqrt{ }$ & $\sqrt{ }$ & $\sqrt{ }$ & - & - & - \\
\hline 47 & $\sqrt{ }$ & & $\checkmark$ & $\sqrt{ }$ & $\sqrt{ }$ & $\sqrt{ }$ & & & $\sqrt{ }$ & $\sqrt{ }$ & $\sqrt{ }$ & $\sqrt{ }$ & -3.5 & -1.8 & 1 \\
\hline 48 & $\sqrt{ }$ & $\sqrt{ }$ & & & & & $\sqrt{ }$ & & & & $\sqrt{ }$ & $\checkmark$ & - & - & - \\
\hline 49 & $\sqrt{ }$ & $\sqrt{ }$ & $\checkmark$ & $\checkmark$ & & & & $\sqrt{ }$ & $\sqrt{ }$ & $\checkmark$ & $\sqrt{ }$ & $\checkmark$ & -1.2 & 5.7 & 1 \\
\hline 50 & & & $\sqrt{ }$ & & & & & & $\sqrt{ }$ & $\sqrt{ }$ & $\checkmark$ & $\sqrt{ }$ & -1.1 & -1.2 & 1 \\
\hline
\end{tabular}


Table 58: Model's change in VTR prediction for worksites with Transit share between $15 \%$ to $25 \%$ and Vehicle Trip Rate between 60 to 70

\begin{tabular}{|c|c|c|c|c|c|c|c|c|c|c|c|c|c|c|c|}
\hline $\begin{array}{l}\text { Sr. } \\
\text { No. }\end{array}$ & $F \& A$ & ONS & СТВ & FI N & CWW & FLEX & TELE & PMT & NONF & GRH & RSMP & MRKT & $\begin{array}{r}\text { Tran } \\
{[0 .]} \\
\text { VTR } \\
\end{array}$ & $\begin{array}{l}\text { nsit Sha } \\
15-0.25 \\
-[70-60\end{array}$ & \\
\hline & & & & & & & & & & & & & Predicted & Actual & No.Ex \\
\hline 1 & $\sqrt{ }$ & $\sqrt{ }$ & $\checkmark$ & $\checkmark$ & & & & & $\sqrt{ }$ & $\sqrt{ }$ & $\sqrt{ }$ & $\sqrt{ }$ & -0.7 & 1.6 & 12 \\
\hline 2 & $\sqrt{ }$ & $\sqrt{ }$ & $\sqrt{ }$ & $\sqrt{ }$ & & $\sqrt{ }$ & & & $\sqrt{ }$ & $\sqrt{ }$ & $\sqrt{ }$ & $\sqrt{ }$ & -0.1 & 5.6 & 9 \\
\hline 3 & $\sqrt{ }$ & $\sqrt{ }$ & $\sqrt{ }$ & & & & & & $\sqrt{ }$ & $\sqrt{ }$ & $\sqrt{ }$ & $\sqrt{ }$ & -0.4 & 1.2 & 7 \\
\hline 4 & $\sqrt{ }$ & & $\sqrt{ }$ & $\sqrt{ }$ & & & & & $\sqrt{ }$ & $\sqrt{ }$ & $\sqrt{ }$ & $\sqrt{ }$ & -2.0 & -3.7 & 4 \\
\hline 5 & $\sqrt{ }$ & $\sqrt{ }$ & & & & & & & $\sqrt{ }$ & $\sqrt{ }$ & $\sqrt{ }$ & $\sqrt{ }$ & 2.0 & 0.4 & 13 \\
\hline 6 & & $\sqrt{ }$ & $\sqrt{ }$ & $\checkmark$ & & & & & $\sqrt{ }$ & $\checkmark$ & $\sqrt{ }$ & $\sqrt{ }$ & -1.1 & 5.4 & 9 \\
\hline 7 & $\sqrt{ }$ & $\sqrt{ }$ & & & & $\sqrt{ }$ & & & $\sqrt{ }$ & $\sqrt{ }$ & $\sqrt{ }$ & $\sqrt{3}$ & 1.0 & 1.3 & 3 \\
\hline 8 & $\sqrt{ }$ & $\sqrt{ }$ & $\checkmark$ & $\sqrt{ }$ & $\sqrt{ }$ & & & & $\sqrt{ }$ & $\sqrt{ }$ & $\sqrt{ }$ & $\sqrt{ }$ & - & - & - \\
\hline 9 & $\sqrt{ }$ & & $\sqrt{ }$ & & & & & & $\sqrt{ }$ & $\sqrt{ }$ & $\sqrt{ }$ & $\sqrt{ }$ & -0.8 & 0.2 & 7 \\
\hline 10 & $\sqrt{ }$ & $\sqrt{ }$ & & & $\sqrt{ }$ & & & & $\sqrt{ }$ & $\sqrt{ }$ & $\sqrt{ }$ & $\sqrt{ }$ & 2.0 & -2.8 & 1 \\
\hline 11 & $\sqrt{ }$ & & $\sqrt{ }$ & $\sqrt{ }$ & & & & & & $\sqrt{ }$ & $\sqrt{ }$ & $\sqrt{ }$ & -2.0 & -3.7 & 4 \\
\hline 12 & $\sqrt{ }$ & & & & & & & & & $\sqrt{ }$ & $\sqrt{ }$ & $\sqrt{ }$ & 1.3 & 2.1 & 7 \\
\hline 13 & $\checkmark$ & $\sqrt{ }$ & $\checkmark$ & & & $\sqrt{ }$ & & & $\sqrt{ }$ & $\sqrt{ }$ & $\checkmark$ & $\checkmark$ & -0.2 & 0.4 & 2 \\
\hline 14 & $\sqrt{ }$ & $\sqrt{ }$ & $\sqrt{ }$ & $\sqrt{ }$ & & & & & & $\sqrt{ }$ & $\sqrt{ }$ & $\checkmark$ & -1.0 & 3.2 & 6 \\
\hline 15 & $\sqrt{ }$ & & & & & & & & $\sqrt{ }$ & $\sqrt{ }$ & $\sqrt{ }$ & $\sqrt{ }$ & 0.4 & -11.8 & 2 \\
\hline 16 & $\sqrt{ }$ & $\sqrt{ }$ & $\sqrt{ }$ & $\checkmark$ & $\sqrt{ }$ & $\sqrt{ }$ & & & $\sqrt{ }$ & $\sqrt{ }$ & $\sqrt{ }$ & $\checkmark$ & 0.4 & -4.5 & 1 \\
\hline 17 & $\sqrt{ }$ & $\sqrt{ }$ & $\sqrt{ }$ & $\sqrt{ }$ & & & & & $\sqrt{ }$ & $\checkmark$ & & $\checkmark$ & -1.4 & 1.5 & 5 \\
\hline 18 & $\sqrt{ }$ & $\sqrt{ }$ & $\sqrt{ }$ & & $\sqrt{ }$ & & & & $\sqrt{ }$ & $\sqrt{ }$ & $\sqrt{ }$ & $\sqrt{ }$ & 3.3 & -4.1 & 1 \\
\hline 19 & $\sqrt{ }$ & & $\sqrt{ }$ & $\sqrt{ }$ & & $\sqrt{ }$ & & & $\sqrt{ }$ & $\sqrt{ }$ & $\sqrt{ }$ & $\sqrt{ }$ & -1.0 & 8.2 & 2 \\
\hline 20 & & & $\sqrt{ }$ & $\sqrt{ }$ & & & & & $\sqrt{ }$ & $\sqrt{ }$ & $\sqrt{ }$ & $\sqrt{ }$ & 0.5 & -0.3 & 6 \\
\hline 21 & $\sqrt{ }$ & $\sqrt{ }$ & & & $\checkmark$ & & $\sqrt{ }$ & & $\sqrt{ }$ & $\checkmark$ & $\checkmark$ & $\sqrt{ }$ & 4.8 & 14.7 & 1 \\
\hline 22 & $\sqrt{ }$ & & $\checkmark$ & & & $\sqrt{ }$ & & & $\sqrt{ }$ & $\sqrt{ }$ & $\sqrt{ }$ & $\sqrt{ }$ & -2.5 & 0.2 & 4 \\
\hline 23 & $\sqrt{ }$ & & & & & & & & & & $\sqrt{ }$ & $\checkmark$ & 1.3 & 8.2 & 1 \\
\hline 24 & $\sqrt{ }$ & $\sqrt{ }$ & 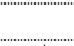 & $\sqrt{ }$ & & . & & & $\sqrt{ }$ & $\sqrt{ }$ & $\sqrt{ }$ & $\checkmark$ & - & - & - \\
\hline 25 & $\sqrt{ }$ & $\sqrt{ }$ & $\sqrt{ }$ & & $\sqrt{ }$ & $\sqrt{ }$ & & & $\sqrt{ }$ & $\sqrt{ }$ & $\sqrt{ }$ & $\sqrt{ }$ & - & - & - \\
\hline 26 & $\sqrt{ }$ & $\sqrt{ }$ & $\sqrt{ }$ & $\sqrt{ }$ & & $\sqrt{ }$ & & & & $\sqrt{ }$ & $\sqrt{ }$ & $\checkmark$ & -0.1 & 0.4 & 1 \\
\hline 27 & $\sqrt{ }$ & $\sqrt{ }$ & & & & & & & & & $\sqrt{ }$ & $\sqrt{ }$ & - & - & - \\
\hline 28 & $\sqrt{ }$ & $\sqrt{ }$ & $\checkmark$ & & $\checkmark$ & & $\sqrt{ }$ & & $\sqrt{ }$ & $\sqrt{ }$ & $\sqrt{ }$ & $\sqrt{ }$ & -0.3 & -13.7 & 1 \\
\hline 29 & $\sqrt{ }$ & $\sqrt{ }$ & $\checkmark$ & & & & & & $\sqrt{ }$ & $\sqrt{ }$ & & $\sqrt{ }$ & -0.2 & 2.4 & 7 \\
\hline 30 & $\sqrt{ }$ & & $\sqrt{ }$ & $\sqrt{ }$ & $\sqrt{ }$ & & & & $\sqrt{ }$ & $\sqrt{ }$ & $\sqrt{ }$ & $\sqrt{ }$ & -0.3 & 1.0 & 1 \\
\hline 31 & $\sqrt{ }$ & $\sqrt{ }$ & - & & & & & & & $\sqrt{ }$ & $\sqrt{ }$ & $\sqrt{ }$ & 0.4 & 0.0 & 6 \\
\hline 32 & $\sqrt{ }$ & & & & & $\sqrt{ }$ & & & $\sqrt{ }$ & $\sqrt{ }$ & $\sqrt{ }$ & $\checkmark$ & -0.9 & 8.8 & 1 \\
\hline 33 & & $\sqrt{ }$ & $\checkmark$ & & & & & & $\sqrt{ }$ & $\sqrt{ }$ & $\sqrt{ }$ & $\checkmark$ & 0.1 & 8.1 & 2 \\
\hline 34 & $\checkmark$ & $\sqrt{ }$ & & $\sqrt{ }$ & $\sqrt{ }$ & $\sqrt{ }$ & & & $\sqrt{ }$ & $\sqrt{ }$ & $\sqrt{ }$ & $\sqrt{ }$ & $=$ & - & - \\
\hline 35 & $\sqrt{ }$ & $\sqrt{ }$ & $\sqrt{ }$ & & & & & & & $\sqrt{ }$ & $\sqrt{ }$ & $\sqrt{ }$ & -0.8 & 2.7 & 6 \\
\hline 36 & $\sqrt{ }$ & & $\sqrt{ }$ & $\checkmark$ & & & & & $\sqrt{ }$ & $\sqrt{ }$ & & $\sqrt{ }$ & -1.6 & -1.9 & 1 \\
\hline 37 & $\sqrt{ }$ & & & $\sqrt{ }$ & & & & & $\sqrt{ }$ & $\sqrt{ }$ & $\sqrt{ }$ & $\sqrt{ }$ & - & - & - \\
\hline 38 & $\checkmark$ & $\sqrt{ }$ & $\checkmark$ & $\checkmark$ & $\checkmark$ & & $\checkmark$ & & $\sqrt{ }$ & $\sqrt{ }$ & $\checkmark$ & $\checkmark$ & - & - & - \\
\hline 39 & $\sqrt{ }$ & $\sqrt{ }$ & $\sqrt{ }$ & $\checkmark$ & $\checkmark$ & $\sqrt{ }$ & $\sqrt{ }$ & & $\sqrt{ }$ & $\checkmark$ & $\sqrt{ }$ & $\sqrt{ }$ & - & - & - \\
\hline 40 & $\sqrt{ }$ & & & $\sqrt{ }$ & & & & & & $\sqrt{ }$ & & $\sqrt{ }$ & - & - & - \\
\hline 41 & $\sqrt{ }$ & $\sqrt{ }$ & & & & & & & $\sqrt{ }$ & & $\sqrt{ }$ & $\sqrt{ }$ & 2.1 & -5.3 & 2 \\
\hline 42 & $\sqrt{ }$ & $\sqrt{ }$ & & & $\checkmark$ & & $\sqrt{ }$ & & & $\sqrt{ }$ & $\sqrt{ }$ & $\sqrt{ }$ & - & - & - \\
\hline 43 & $\sqrt{ }$ & $\sqrt{ }$ & & & $\sqrt{ }$ & & $\checkmark$ & & $\sqrt{ }$ & & $\checkmark$ & $\checkmark$ & 2.7 & -6.3 & 2 \\
\hline 44 & $\sqrt{ }$ & $\sqrt{ }$ & $\sqrt{ }$ & $\sqrt{ }$ & & & $\sqrt{ }$ & & $\sqrt{ }$ & $\sqrt{ }$ & $\sqrt{ }$ & $\sqrt{ }$ & 0.0 & 4.7 & 1 \\
\hline 45 & $\sqrt{ }$ & $\sqrt{ }$ & & & $\sqrt{ }$ & & & & & & $\sqrt{ }$ & $\sqrt{ }$ & - & - & - \\
\hline 46 & $\sqrt{ }$ & & & & $\sqrt{ }$ & $\sqrt{ }$ & & & & $\sqrt{ }$ & $\sqrt{ }$ & $\sqrt{ }$ & - & - & - \\
\hline 47 & $\sqrt{ }$ & & $\checkmark$ & $\sqrt{ }$ & $\sqrt{ }$ & $\sqrt{ }$ & & & $\sqrt{ }$ & $\sqrt{ }$ & $\sqrt{ }$ & $\sqrt{ }$ & - & - & - \\
\hline 48 & $\sqrt{ }$ & $\checkmark$ & & & & & $\sqrt{ }$ & & & & $\sqrt{ }$ & $\checkmark$ & -3.7 & -31.7 & 1 \\
\hline 49 & $\sqrt{ }$ & $\sqrt{ }$ & $\checkmark$ & $\checkmark$ & & & & $\sqrt{ }$ & $\sqrt{ }$ & $\checkmark$ & $\checkmark$ & $\checkmark$ & -1.7 & -1.8 & 5 \\
\hline 50 & & & $\sqrt{ }$ & & & & & & $\sqrt{ }$ & $\sqrt{ }$ & $\checkmark$ & $\sqrt{ }$ & -0.4 & 1.1 & 4 \\
\hline
\end{tabular}


Table 59: Model's change in VTR prediction for worksites with Transit share between $15 \%$ to $25 \%$ and Vehicle Trip Rate between 50 to 60

\begin{tabular}{|c|c|c|c|c|c|c|c|c|c|c|c|c|c|c|c|}
\hline \multirow[t]{2}{*}{$\begin{array}{l}\text { Sr. } \\
\text { No. }\end{array}$} & \multirow[t]{2}{*}{ F\&A } & \multirow[t]{2}{*}{ ONS } & \multirow[t]{2}{*}{ СТВ } & \multirow[t]{2}{*}{ FIN } & \multirow[t]{2}{*}{ CWw } & \multirow[t]{2}{*}{ FLEX } & \multirow[t]{2}{*}{ TELE } & \multirow[t]{2}{*}{ PMT } & \multirow[t]{2}{*}{ NONF } & \multirow[t]{2}{*}{ GRH } & \multirow[t]{2}{*}{ RSMP } & \multirow[t]{2}{*}{ MRKT } & \multicolumn{3}{|c|}{$\begin{array}{c}\text { Transit Share } \\
{[0.15-0.25)} \\
\text { VTR- }[60-50)\end{array}$} \\
\hline & & & & & & & & & & & & & Predicted & Actual & No.Ex \\
\hline 1 & $\sqrt{ }$ & $\sqrt{ }$ & $\sqrt{ }$ & $\sqrt{ }$ & & & & & $\sqrt{ }$ & $\sqrt{ }$ & $\sqrt{ }$ & $\sqrt{ }$ & 2.4 & 26.4 & 2 \\
\hline 2 & $\sqrt{ }$ & $\sqrt{ }$ & $\sqrt{ }$ & $\sqrt{ }$ & & $\sqrt{ }$ & & & $\sqrt{ }$ & $\sqrt{ }$ & $\checkmark$ & $\sqrt{ }$ & 0.9 & 6.8 & 1 \\
\hline 3 & $\sqrt{ }$ & $\sqrt{ }$ & v & & & & & & $\sqrt{ }$ & $\sqrt{ }$ & V & V & -0.3 & -4.3 & 1 \\
\hline 4 & $\sqrt{ }$ & & $\sqrt{ }$ & $\sqrt{ }$ & & & & & $\sqrt{ }$ & $\sqrt{ }$ & $\sqrt{ }$ & $\sqrt{ }$ & - & - & - \\
\hline 5 & $\sqrt{ }$ & $\sqrt{ }$ & & & & & & & $\sqrt{ }$ & $\sqrt{ }$ & $\sqrt{ }$ & $\sqrt{ }$ & 2.3 & -1.5 & 4 \\
\hline 6 & & $\sqrt{ }$ & $\checkmark$ & $\sqrt{ }$ & & & & & $\sqrt{ }$ & $\sqrt{ }$ & $\checkmark$ & $\checkmark$ & - & - & - \\
\hline 7 & $\sqrt{ }$ & $\sqrt{ }$ & & & & $\sqrt{ }$ & & & $\sqrt{ }$ & $\sqrt{ }$ & $\sqrt{ }$ & $\sqrt{ }$ & 4.4 & 11.5 & 2 \\
\hline 8 & $\sqrt{ }$ & $\sqrt{ }$ & $\sqrt{ }$ & $\sqrt{ }$ & $\sqrt{ }$ & & & & $\sqrt{ }$ & $\sqrt{ }$ & $\sqrt{ }$ & $\sqrt{ }$ & - & - & - \\
\hline 9 & $\sqrt{ }$ & & $\sqrt{ }$ & & & & & & $\sqrt{ }$ & $\sqrt{ }$ & $\sqrt{ }$ & $\sqrt{ }$ & 0.2 & 5.2 & 2 \\
\hline 10 & $\sqrt{ }$ & $\sqrt{ }$ & & & $\sqrt{ }$ & & & & $\sqrt{ }$ & $\sqrt{ }$ & $\sqrt{ }$ & $\sqrt{ }$ & - & - & - \\
\hline 11 & $\sqrt{ }$ & & $\sqrt{ }$ & $\sqrt{ }$ & & & & & & $\sqrt{ }$ & $\sqrt{ }$ & $\sqrt{ }$ & - & - & - \\
\hline 12 & $\sqrt{ }$ & & & & & & & & & $\sqrt{ }$ & $\sqrt{ }$ & $\sqrt{ }$ & 3.3 & 9.9 & 2 \\
\hline 13 & $\checkmark$ & $\sqrt{ }$ & $\checkmark$ & & & $\sqrt{ }$ & & & $\sqrt{ }$ & $\sqrt{ }$ & $\checkmark$ & $\checkmark$ & - & - & - \\
\hline 14 & $\sqrt{ }$ & $\sqrt{ }$ & $\sqrt{ }$ & $\sqrt{ }$ & & & & & & $\sqrt{ }$ & $\sqrt{ }$ & $\sqrt{ }$ & - & - & - \\
\hline 15 & $\sqrt{ }$ & & & & & & & & $\sqrt{ }$ & $\sqrt{ }$ & $\sqrt{ }$ & $\sqrt{ }$ & 4.2 & 0.3 & 3 \\
\hline 16 & $\sqrt{ }$ & $\sqrt{ }$ & $\sqrt{ }$ & $\sqrt{ }$ & $\sqrt{ }$ & $\sqrt{ }$ & & & $\sqrt{ }$ & $\sqrt{ }$ & $\sqrt{ }$ & $\sqrt{ }$ & - & - & - \\
\hline 17 & $\sqrt{ }$ & $\sqrt{ }$ & $\sqrt{ }$ & $\sqrt{ }$ & & & & & $\sqrt{ }$ & $\sqrt{ }$ & ........... & $\sqrt{ }$ & - & - & - \\
\hline 18 & $\sqrt{ }$ & $\sqrt{ }$ & v & & v & & & & $\sqrt{ }$ & $\sqrt{ }$ & V & $\sqrt{ }$ & - & - & - \\
\hline 19 & $\sqrt{ }$ & & $\sqrt{ }$ & $\sqrt{ }$ & & $\sqrt{ }$ & & & $\sqrt{ }$ & $\sqrt{ }$ & $\sqrt{ }$ & $\sqrt{ }$ & - & - & - \\
\hline 20 & & & $\sqrt{ }$ & $\sqrt{ }$ & & & & & $\sqrt{ }$ & $\sqrt{ }$ & $\sqrt{ }$ & $\sqrt{ }$ & - & - & - \\
\hline 21 & $\sqrt{ }$ & $\sqrt{ }$ & & & $\sqrt{ }$ & & $\sqrt{ }$ & & $\sqrt{ }$ & $\sqrt{ }$ & $\sqrt{ }$ & V & - & - & - \\
\hline 22 & $\sqrt{ }$ & & $\sqrt{ }$ & & & $\sqrt{ }$ & & & $\sqrt{ }$ & $\sqrt{ }$ & $\sqrt{ }$ & $\sqrt{ }$ & 2.4 & 14.9 & 1 \\
\hline 23 & $\sqrt{ }$ & & & & & & & & & & $\sqrt{ }$ & $\sqrt{ }$ & - & - & - \\
\hline 24 & $\sqrt{ }$ & $\sqrt{ }$ & 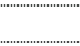 & $\sqrt{ }$ & & & & & $\sqrt{ }$ & $\sqrt{ }$ & $\sqrt{ }$ & $\sqrt{ }$ & - & - & - \\
\hline 25 & $\sqrt{ }$ & $\sqrt{ }$ & $\sqrt{ }$ & & $\sqrt{ }$ & $\sqrt{ }$ & & & $\sqrt{ }$ & $\sqrt{ }$ & $\sqrt{ }$ & $\sqrt{ }$ & - & - & - \\
\hline 26 & $\sqrt{ }$ & $\sqrt{ }$ & $\sqrt{ }$ & $\sqrt{ }$ & & $\sqrt{ }$ & & & & $\sqrt{ }$ & $\sqrt{ }$ & $\sqrt{ }$ & - & - & - \\
\hline 27 & $\sqrt{ }$ & $\sqrt{ }$ & & & & & & & & 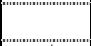 & $\checkmark$ & $\checkmark$ & 1.0 & 0.0 & 1 \\
\hline 28 & $\sqrt{ }$ & $\sqrt{ }$ & $\checkmark$ & & $\sqrt{ }$ & & $\sqrt{ }$ & & $\sqrt{ }$ & $\sqrt{ }$ & $\sqrt{ }$ & V & - & - & - \\
\hline 29 & $\sqrt{ }$ & $\sqrt{ }$ & $\sqrt{ }$ & & & & & & $\sqrt{ }$ & $\sqrt{ }$ & & $\sqrt{ }$ & - & - & - \\
\hline 30 & $\sqrt{ }$ & & $\sqrt{ }$ & $\sqrt{ }$ & $\sqrt{ }$ & & & & $\sqrt{ }$ & $\sqrt{ }$ & $\sqrt{ }$ & $\sqrt{ }$ & - & - & - \\
\hline 31 & $\sqrt{ }$ & $\sqrt{ }$ & & & & & & & & $\sqrt{ }$ & $\sqrt{ }$ & $\sqrt{ }$ & 3.2 & -3.4 & 4 \\
\hline 32 & $\sqrt{ }$ & & & & & $\sqrt{ }$ & & & $\sqrt{ }$ & $\sqrt{ }$ & $\sqrt{ }$ & $\sqrt{ }$ & - & - & - \\
\hline 33 & & $\sqrt{ }$ & $\sqrt{ }$ & & & & & & $\sqrt{ }$ & $\sqrt{ }$ & $\sqrt{ }$ & $\sqrt{ }$ & -2.0 & -12.5 & 1 \\
\hline 34 & $\sqrt{ }$ & $\sqrt{ }$ & & $\sqrt{ }$ & $\sqrt{ }$ & $\sqrt{ }$ & & & $\sqrt{ }$ & $\sqrt{ }$ & $\sqrt{ }$ & $\sqrt{ }$ & - & - & - \\
\hline 35 & $\sqrt{ }$ & $\sqrt{ }$ & $\sqrt{ }$ & & & & & & & $\sqrt{ }$ & $\sqrt{ }$ & $\sqrt{ }$ & 3.8 & 4.8 & 1 \\
\hline 36 & $\sqrt{ }$ & & $\sqrt{ }$ & $\sqrt{ }$ & & & & & $\sqrt{ }$ & $\sqrt{ }$ & & $\sqrt{ }$ & - & - & - \\
\hline 37 & $\sqrt{ }$ & & & $\sqrt{ }$ & & & & & $\sqrt{ }$ & $\sqrt{ }$ & $\sqrt{ }$ & $\sqrt{ }$ & - & - & - \\
\hline 38 & $\sqrt{ }$ & $\sqrt{ }$ & V & $\sqrt{ }$ & V & & $\sqrt{ }$ & & $\sqrt{ }$ & $\sqrt{ }$ & $\sqrt{ }$ & $\checkmark$ & - & - & - \\
\hline 39 & $\sqrt{ }$ & $\sqrt{ }$ & $\sqrt{ }$ & $\sqrt{ }$ & $\sqrt{ }$ & $\sqrt{ }$ & $\sqrt{ }$ & & $\sqrt{ }$ & $\sqrt{ }$ & $\sqrt{ }$ & $\sqrt{ }$ & - & - & - \\
\hline 40 & $\sqrt{ }$ & & & $\sqrt{ }$ & & & & & & $\sqrt{ }$ & 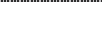 & $\sqrt{ }$ & - & - & - \\
\hline 41 & $\sqrt{ }$ & $\sqrt{ }$ & & & & & & & $\sqrt{ }$ & & $\sqrt{ }$ & $\sqrt{ }$ & 3.2 & 11.7 & 1 \\
\hline 42 & $\sqrt{ }$ & $\sqrt{ }$ & & & $\sqrt{ }$ & & $\sqrt{ }$ & & & $\sqrt{ }$ & $\sqrt{ }$ & $\sqrt{ }$ & - & - & - \\
\hline 43 & $\checkmark$ & $\sqrt{ }$ & & & $\checkmark$ & & $\sqrt{ }$ & & $\sqrt{ }$ & & $\sqrt{ }$ & $\checkmark$ & - & - & - \\
\hline 44 & $\sqrt{ }$ & $\sqrt{ }$ & $\sqrt{ }$ & $\sqrt{ }$ & & & $\sqrt{ }$ & & $\sqrt{ }$ & $\sqrt{ }$ & $\sqrt{ }$ & $\sqrt{ }$ & - & - & - \\
\hline 45 & $\sqrt{ }$ & $\sqrt{ }$ & & & $\sqrt{ }$ & & & & & & $\sqrt{ }$ & $\sqrt{ }$ & 1.1 & -5.4 & 1 \\
\hline 46 & $\sqrt{ }$ & & & & $\sqrt{ }$ & $\sqrt{ }$ & & & & $\sqrt{ }$ & $\sqrt{ }$ & $\sqrt{ }$ & 8.0 & 21.1 & 1 \\
\hline 47 & $\sqrt{ }$ & & $\sqrt{ }$ & $\sqrt{ }$ & $\sqrt{ }$ & $\sqrt{ }$ & & & $\sqrt{ }$ & $\sqrt{ }$ & $\sqrt{ }$ & $\sqrt{ }$ & - & - & - \\
\hline 48 & $\sqrt{ }$ & $\sqrt{ }$ & & & & & $\sqrt{ }$ & & & & $\checkmark$ & $\checkmark$ & -4.6 & 22.1 & 1 \\
\hline 49 & $\sqrt{ }$ & $\sqrt{ }$ & $\sqrt{ }$ & $\sqrt{ }$ & & & & $\sqrt{ }$ & $\sqrt{ }$ & $\sqrt{ }$ & $\checkmark$ & $\sqrt{ }$ & 0.3 & -2.6 & 3 \\
\hline 50 & & & $\sqrt{ }$ & & & & & & $\sqrt{ }$ & V & $\sqrt{ }$ & $\sqrt{ }$ & - & - & - \\
\hline
\end{tabular}


Table 60: Model's change in VTR prediction for worksites with Transit share between $25 \%$ to $35 \%$ and Vehicle Trip Rate between 60 to 70

\begin{tabular}{|c|c|c|c|c|c|c|c|c|c|c|c|c|c|c|c|}
\hline $\begin{array}{l}\text { Sr. } \\
\text { No. }\end{array}$ & $F \& A$ & ONS & СТВ & FIN & CWw & FLEX & TELE & PMT & NONF & GRH & RSMP & MRKT & $\begin{array}{c}\text { Trar } \\
{[0 .} \\
\text { VTR }\end{array}$ & $\begin{array}{l}\text { ssit Sha } \\
25-0.35 \\
-[70-6\end{array}$ & \\
\hline & & & & & & & & & & & & & Predicted & Actual & No.Ex \\
\hline 1 & $\sqrt{ }$ & $\sqrt{ }$ & $\sqrt{ }$ & $\sqrt{ }$ & & & & & $\sqrt{ }$ & $\sqrt{ }$ & $\sqrt{ }$ & $\sqrt{ }$ & -1.9 & 7.2 & 3 \\
\hline 2 & $\sqrt{ }$ & $\sqrt{ }$ & $\sqrt{ }$ & $\sqrt{ }$ & & $\sqrt{ }$ & & & $\sqrt{ }$ & $\sqrt{ }$ & $\sqrt{ }$ & $\sqrt{ }$ & -1.9 & -3.9 & 4 \\
\hline 3 & $\checkmark$ & $\sqrt{ }$ & $\sqrt{ }$ & & & & & & $\sqrt{ }$ & $\checkmark$ & $\sqrt{ }$ & $\sqrt{ }$ & -3.0 & -7.3 & 1 \\
\hline 4 & $\sqrt{ }$ & & $\sqrt{ }$ & $\sqrt{ }$ & & & & & $\sqrt{ }$ & $\sqrt{ }$ & $\sqrt{ }$ & $\sqrt{ }$ & -1.9 & 12.8 & 1 \\
\hline 5 & $\sqrt{ }$ & $\sqrt{ }$ & & & & & & & $\sqrt{ }$ & $\sqrt{ }$ & $\sqrt{ }$ & $\sqrt{ }$ & - & - & - \\
\hline 6 & & $\sqrt{ }$ & $\sqrt{ }$ & $\sqrt{ }$ & & & & & $\sqrt{ }$ & $\sqrt{ }$ & $\sqrt{ }$ & V & -2.9 & -4.4 & 3 \\
\hline 7 & $\sqrt{ }$ & $\sqrt{ }$ & & & & $\sqrt{ }$ & & & $\sqrt{ }$ & $\checkmark$ & $\sqrt{ }$ & $\sqrt{ }$ & $=$ & - & - \\
\hline 8 & $\sqrt{ }$ & $\sqrt{ }$ & $\sqrt{ }$ & $\sqrt{ }$ & $\sqrt{ }$ & & & & $\sqrt{ }$ & v & $\sqrt{ }$ & $\sqrt{ }$ & - & - & - \\
\hline 9 & $\sqrt{ }$ & & $\checkmark$ & & & & & & $\sqrt{ }$ & $\sqrt{ }$ & $\sqrt{ }$ & $\sqrt{ }$ & -1.8 & -0.4 & 3 \\
\hline 10 & $\sqrt{ }$ & $\sqrt{ }$ & & & $\sqrt{ }$ & & & & $\sqrt{ }$ & $\sqrt{ }$ & $\sqrt{ }$ & $\sqrt{ }$ & - & - & - \\
\hline 11 & $\sqrt{ }$ & & $\sqrt{ }$ & $\checkmark$ & & & & & & $\sqrt{ }$ & $\sqrt{ }$ & $\sqrt{ }$ & -1.9 & 12.8 & 1 \\
\hline 12 & $\sqrt{ }$ & & & & & & & & & $\sqrt{ }$ & $\sqrt{ }$ & $\sqrt{ }$ & -3.3 & -8.2 & 2 \\
\hline 13 & $\sqrt{ }$ & $\sqrt{ }$ & $\sqrt{ }$ & & & $\sqrt{ }$ & & & $\sqrt{ }$ & $\sqrt{ }$ & $\sqrt{ }$ & $\sqrt{ }$ & -3.1 & 1.5 & 1 \\
\hline 14 & $\sqrt{ }$ & $\sqrt{ }$ & $\sqrt{ }$ & $\checkmark$ & & & & & & $\checkmark$ & $\checkmark$ & $\sqrt{ }$ & -2.5 & -3.0 & 2 \\
\hline 15 & $\sqrt{ }$ & & & & & & & & $\sqrt{ }$ & $\sqrt{ }$ & $\sqrt{ }$ & $\sqrt{ }$ & - & - & - \\
\hline 16 & $\sqrt{ }$ & $\sqrt{ }$ & $\sqrt{ }$ & $\sqrt{ }$ & $\sqrt{ }$ & $\sqrt{ }$ & & & $\sqrt{ }$ & $\sqrt{ }$ & $\sqrt{ }$ & $\sqrt{ }$ & - & - & - \\
\hline 17 & $\sqrt{ }$ & $\sqrt{ }$ & $\sqrt{ }$ & $\sqrt{ }$ & & & & & $\sqrt{ }$ & $\sqrt{ }$ & & $\checkmark$ & - & - & - \\
\hline 18 & $\sqrt{ }$ & $\sqrt{ }$ & $\checkmark$ & & $\checkmark$ & & & & $\sqrt{ }$ & $\checkmark$ & $\sqrt{ }$ & $\checkmark$ & -1.9 & 1.5 & 1 \\
\hline 19 & $\sqrt{ }$ & & $\sqrt{ }$ & $\sqrt{ }$ & & $\sqrt{ }$ & & & $\sqrt{ }$ & $\sqrt{ }$ & $\sqrt{ }$ & $\sqrt{ }$ & - & - & - \\
\hline 20 & & & $\sqrt{ }$ & $\sqrt{ }$ & & & & & $\sqrt{ }$ & $\sqrt{ }$ & $\sqrt{ }$ & $\sqrt{ }$ & - & - & - \\
\hline 21 & $\sqrt{ }$ & $\sqrt{ }$ & & & $\sqrt{ }$ & & $\sqrt{ }$ & & $\sqrt{ }$ & $\sqrt{ }$ & $\sqrt{ }$ & $\sqrt{ }$ & - & - & - \\
\hline 22 & $\sqrt{ }$ & & $\sqrt{ }$ & & & $\sqrt{ }$ & & & $\sqrt{ }$ & $\sqrt{ }$ & $\sqrt{ }$ & $\sqrt{ }$ & - & - & - \\
\hline 23 & $\sqrt{ }$ & & & & & & & & & & $\sqrt{ }$ & $\sqrt{ }$ & - & - & - \\
\hline 24 & $\sqrt{ }$ & $\sqrt{ }$ & & $\checkmark$ & & & & & $\sqrt{ }$ & $\sqrt{ }$ & $\sqrt{ }$ & $\checkmark$ & - & - & - \\
\hline 25 & $\sqrt{ }$ & $\sqrt{ }$ & $\checkmark$ & $x^{\prime \prime}$ & $\sqrt{ }$ & $\sqrt{ }$ & & & $\sqrt{ }$ & $\sqrt{ }$ & $\checkmark$ & $\checkmark$ & - & - & - \\
\hline 26 & $\sqrt{ }$ & $\sqrt{ }$ & $\sqrt{ }$ & $\sqrt{ }$ & & $\sqrt{ }$ & & & & $\sqrt{ }$ & $\sqrt{ }$ & $\sqrt{ }$ & -3.2 & -8.5 & 1 \\
\hline 27 & $\sqrt{ }$ & $\sqrt{ }$ & & & & & & & & & $\sqrt{ }$ & J & - & - & - \\
\hline 28 & $V$ & $\sqrt{ }$ & $\checkmark$ & & $\checkmark$ & & $\checkmark$ & & $\sqrt{ }$ & $\sqrt{ }$ & $\sqrt{ }$ & $\sqrt{ }$ & $=$ & - & - \\
\hline 29 & $\sqrt{ }$ & $\sqrt{ }$ & $\sqrt{ }$ & & & & & & $\sqrt{ }$ & $\sqrt{ }$ & & $\sqrt{ }$ & -2.4 & 4.5 & 1 \\
\hline 30 & $\sqrt{ }$ & & $\sqrt{ }$ & $\sqrt{ }$ & $\sqrt{ }$ & & & & $\sqrt{ }$ & $\sqrt{ }$ & $\sqrt{ }$ & $\sqrt{ }$ & - & - & - \\
\hline 31 & $\sqrt{ }$ & $\sqrt{ }$ & & & & & & & & $\sqrt{ }$ & $\sqrt{ }$ & $\sqrt{ }$ & -1.4 & -6.0 & 1 \\
\hline 32 & $\sqrt{ }$ & & & & & $\sqrt{ }$ & & & $\sqrt{ }$ & $\sqrt{ }$ & $\sqrt{ }$ & $\checkmark$ & -2.6 & 6.7 & 1 \\
\hline 33 & & $\sqrt{ }$ & $\checkmark$ & & & & & & $\sqrt{ }$ & $\checkmark$ & $\sqrt{ }$ & $\sqrt{ }$ & - & - & - \\
\hline 34 & $\sqrt{ }$ & $\sqrt{ }$ & & $\sqrt{ }$ & $\sqrt{ }$ & $\sqrt{ }$ & & & $\sqrt{ }$ & $\sqrt{ }$ & $\sqrt{ }$ & $\sqrt{ }$ & - & - & - \\
\hline 35 & $\sqrt{ }$ & $\sqrt{ }$ & $\sqrt{ }$ & & & & & & & $\sqrt{ }$ & $\sqrt{ }$ & $\sqrt{ }$ & -1.7 & -7.3 & 2 \\
\hline 36 & $\sqrt{ }$ & & $\sqrt{ }$ & $\sqrt{ }$ & & & & & $\sqrt{ }$ & $\sqrt{ }$ & & $\sqrt{ }$ & - & - & - \\
\hline 37 & $\sqrt{ }$ & & & $\sqrt{ }$ & & & & & $\sqrt{ }$ & $\sqrt{ }$ & $\sqrt{ }$ & $\sqrt{ }$ & - & - & - \\
\hline 38 & $\sqrt{ }$ & $\sqrt{ }$ & $\sqrt{ }$ & $\checkmark$ & $\sqrt{ }$ & & $\sqrt{ }$ & & $\sqrt{ }$ & $\sqrt{ }$ & $\sqrt{ }$ & $\sqrt{ }$ & - & - & - \\
\hline 39 & $\sqrt{ }$ & $\sqrt{ }$ & $\checkmark$ & $\checkmark$ & $\checkmark$ & $\sqrt{ }$ & $\sqrt{ }$ & & $\sqrt{ }$ & $\checkmark$ & $\sqrt{ }$ & $\checkmark$ & 2.1 & -6.7 & 1 \\
\hline 40 & $\sqrt{ }$ & & & $\sqrt{ }$ & & & & & & $\sqrt{ }$ & & $\sqrt{ }$ & - & - & - \\
\hline 41 & $\sqrt{ }$ & $\sqrt{ }$ & & & & & & & $\sqrt{ }$ & & $\sqrt{ }$ & $\sqrt{ }$ & - & - & - \\
\hline 42 & $\sqrt{ }$ & $\sqrt{ }$ & & & $\checkmark$ & & $\checkmark$ & & & $\sqrt{ }$ & $\sqrt{ }$ & $\checkmark$ & - & - & - \\
\hline 43 & $\sqrt{ }$ & $\sqrt{ }$ & & & $\sqrt{ }$ & & $\sqrt{ }$ & & $\sqrt{ }$ & & $\sqrt{ }$ & $\sqrt{ }$ & - & - & - \\
\hline 44 & $\checkmark$ & $\sqrt{ }$ & $\checkmark$ & $\checkmark$ & & & $\checkmark$ & & $\sqrt{ }$ & $\sqrt{ }$ & $\checkmark$ & $\checkmark$ & - & - & - \\
\hline 45 & $\sqrt{ }$ & $\sqrt{ }$ & & & $\sqrt{ }$ & & & & & & $\sqrt{ }$ & $\sqrt{ }$ & - & - & - \\
\hline 46 & $\sqrt{ }$ & 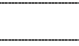 & & & $\sqrt{ }$ & $\sqrt{ }$ & & & & $\sqrt{ }$ & $\sqrt{ }$ & $\checkmark$ & - & - & - \\
\hline 47 & $\sqrt{ }$ & & $\sqrt{ }$ & $\sqrt{ }$ & $\sqrt{ }$ & $\sqrt{ }$ & & & $\sqrt{ }$ & $\sqrt{ }$ & $\sqrt{ }$ & $\sqrt{ }$ & - & - & - \\
\hline 48 & $\sqrt{ }$ & $\sqrt{ }$ & & & & & $\sqrt{ }$ & & & & $\sqrt{ }$ & $\checkmark$ & - & - & - \\
\hline 49 & $\sqrt{ }$ & $\sqrt{ }$ & $\sqrt{ }$ & $\checkmark$ & & & & $\sqrt{ }$ & $\sqrt{ }$ & $V$ & $\checkmark$ & $\checkmark$ & -1.5 & -0.1 & 1 \\
\hline 50 & & & $\sqrt{ }$ & & & & & & $\sqrt{ }$ & $\sqrt{ }$ & $\sqrt{ }$ & $\sqrt{ }$ & - & - & - \\
\hline
\end{tabular}


Table 61: Model's change in VTR prediction for worksites with Transit share between $25 \%$ to $35 \%$ and Vehicle Trip Rate between 50 to 60

\begin{tabular}{|c|c|c|c|c|c|c|c|c|c|c|c|c|c|c|c|}
\hline $\begin{array}{l}\text { Sr. } \\
\text { No. }\end{array}$ & $F \& A$ & ONS & СТВ & FI N & CWW & FLEX & TELE & PMT & NONF & GRH & RSMP & MRKT & $\begin{array}{r}\text { Trar } \\
{[0 .} \\
\text { VTR } \\
\end{array}$ & $\begin{array}{l}\text { Isit Sha } \\
25-0.35 \\
-[60-5\end{array}$ & \\
\hline & & & & & & & & & & & & & Predicted & Actual & No.Ex \\
\hline 1 & $\sqrt{ }$ & $\sqrt{ }$ & $\sqrt{ }$ & $\sqrt{ }$ & & & & & $\sqrt{ }$ & $\sqrt{ }$ & $\sqrt{ }$ & $\sqrt{ }$ & -0.5 & -0.3 & 5 \\
\hline 2 & $\sqrt{ }$ & $\sqrt{ }$ & $\sqrt{ }$ & $\sqrt{ }$ & & $\sqrt{ }$ & & & $\sqrt{ }$ & $\sqrt{ }$ & $\sqrt{ }$ & $\sqrt{ }$ & - & - & - \\
\hline 3 & $\sqrt{ }$ & $\sqrt{ }$ & $\sqrt{ }$ & & & & & & $\sqrt{ }$ & $\sqrt{ }$ & v & $\sqrt{ }$ & 0.6 & 4.9 & 3 \\
\hline 4 & $\sqrt{ }$ & & $\sqrt{ }$ & $\sqrt{ }$ & & & & & $\sqrt{ }$ & $\sqrt{ }$ & $\sqrt{ }$ & $\sqrt{ }$ & - & - & - \\
\hline 5 & $\sqrt{ }$ & $\sqrt{ }$ & & & & & & & $\sqrt{ }$ & $\sqrt{ }$ & $\sqrt{ }$ & $\sqrt{ }$ & - & - & - \\
\hline 6 & & $\sqrt{ }$ & $\sqrt{ }$ & $\sqrt{ }$ & & & & & $\sqrt{ }$ & $\sqrt{ }$ & $\sqrt{ }$ & $\sqrt{ }$ & 0.1 & 1.5 & 3 \\
\hline 7 & $\sqrt{ }$ & $\sqrt{ }$ & & & & $\sqrt{ }$ & & & $\sqrt{ }$ & $\sqrt{ }$ & $\sqrt{ }$ & $\checkmark$ & -1.9 & 6.8 & 1 \\
\hline 8 & $\sqrt{ }$ & $\sqrt{ }$ & $\sqrt{ }$ & $\sqrt{ }$ & $\sqrt{ }$ & & & & $\sqrt{ }$ & $\sqrt{ }$ & $\sqrt{ }$ & $\sqrt{ }$ & - & - & - \\
\hline 9 & $\sqrt{ }$ & & $\sqrt{ }$ & & & & & & $\sqrt{ }$ & $\sqrt{ }$ & $\sqrt{ }$ & $\sqrt{ }$ & -1.4 & 0.0 & 2 \\
\hline 10 & $\sqrt{ }$ & $\sqrt{ }$ & & & $\sqrt{ }$ & & & & $\sqrt{ }$ & $\sqrt{ }$ & $\sqrt{ }$ & $\sqrt{ }$ & - & - & - \\
\hline 11 & $\sqrt{ }$ & & $\sqrt{ }$ & $\sqrt{ }$ & & & & & & $\sqrt{ }$ & $\sqrt{ }$ & $\sqrt{ }$ & - & - & - \\
\hline 12 & $\sqrt{ }$ & & & & & & & & & $\sqrt{ }$ & $\sqrt{ }$ & $\sqrt{ }$ & - & - & - \\
\hline 13 & $\sqrt{ }$ & $\sqrt{ }$ & $\sqrt{ }$ & & & $\sqrt{ }$ & & & $\sqrt{ }$ & $\sqrt{ }$ & $\sqrt{ }$ & $\sqrt{ }$ & 1.3 & 2.7 & 1 \\
\hline 14 & $\sqrt{ }$ & $\sqrt{ }$ & $\sqrt{ }$ & $\sqrt{ }$ & & & & & & $\sqrt{ }$ & $\sqrt{ }$ & $\checkmark$ & 1.5 & 0.1 & 1 \\
\hline 15 & $\sqrt{ }$ & & & & & & & & $\sqrt{ }$ & $\sqrt{ }$ & $\sqrt{ }$ & $\sqrt{ }$ & 1.5 & 0.6 & 3 \\
\hline 16 & $\sqrt{ }$ & $\sqrt{ }$ & $\sqrt{ }$ & $\sqrt{ }$ & $\sqrt{ }$ & $\sqrt{ }$ & & & $\sqrt{ }$ & $\sqrt{ }$ & $\sqrt{ }$ & $\sqrt{ }$ & - & - & - \\
\hline 17 & $\sqrt{ }$ & $\sqrt{ }$ & $\checkmark$ & $\sqrt{ }$ & & & & & $\sqrt{ }$ & $\sqrt{ }$ & $x^{\prime \prime}$ & $\checkmark$ & - & - & - \\
\hline 18 & $\sqrt{ }$ & $\sqrt{ }$ & $\sqrt{ }$ & & $\sqrt{ }$ & & & & $\sqrt{ }$ & $\sqrt{ }$ & $\sqrt{ }$ & $\sqrt{ }$ & - & - & - \\
\hline 19 & $\sqrt{ }$ & & $\sqrt{ }$ & $\sqrt{ }$ & & $\sqrt{ }$ & & & $\sqrt{ }$ & $\sqrt{ }$ & $\sqrt{ }$ & $\sqrt{ }$ & -0.5 & 4.2 & 1 \\
\hline 20 & & & $\sqrt{ }$ & $\sqrt{ }$ & & & & & $\sqrt{ }$ & $\sqrt{ }$ & $\sqrt{ }$ & $\sqrt{ }$ & 0.8 & -2.4 & 1 \\
\hline 21 & $\sqrt{ }$ & $\sqrt{ }$ & & & $\sqrt{ }$ & & $\sqrt{ }$ & & $\sqrt{ }$ & $\sqrt{ }$ & $\sqrt{ }$ & $\sqrt{ }$ & 5.3 & 10.8 & 1 \\
\hline 22 & $\sqrt{ }$ & & $\sqrt{ }$ & & & $\sqrt{ }$ & & & $\sqrt{ }$ & $\sqrt{ }$ & $\sqrt{ }$ & $\sqrt{ }$ & - & - & - \\
\hline 23 & $\sqrt{ }$ & & 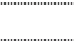 & & & & & & & & $\sqrt{ }$ & $\sqrt{ }$ & - & - & - \\
\hline 24 & $\sqrt{ }$ & $\sqrt{ }$ & $=$ & $\sqrt{ }$ & & . & & & $\sqrt{ }$ & $\sqrt{ }$ & $\sqrt{ }$ & $\sqrt{ }$ & - & - & - \\
\hline 25 & $\sqrt{ }$ & $\sqrt{ }$ & $\sqrt{ }$ & & $\sqrt{ }$ & $\sqrt{ }$ & & & $\sqrt{ }$ & $\sqrt{ }$ & $\sqrt{ }$ & $\sqrt{ }$ & -1.8 & 29.3 & 1 \\
\hline 26 & $\sqrt{ }$ & $\sqrt{ }$ & $\sqrt{ }$ & $\sqrt{ }$ & & $\sqrt{ }$ & & & & $\sqrt{ }$ & $\sqrt{ }$ & $\checkmark$ & - & - & - \\
\hline 27 & $\checkmark$ & $\sqrt{ }$ & & & & & & & & 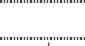 & $\sqrt{ }$ & $\sqrt{ }$ & 3.2 & 0.1 & 3 \\
\hline 28 & $\sqrt{ }$ & $\sqrt{ }$ & $\sqrt{ }$ & & $\sqrt{ }$ & & $\sqrt{ }$ & & $\sqrt{ }$ & $\sqrt{ }$ & $\sqrt{ }$ & $\sqrt{ }$ & - & - & - \\
\hline 29 & $\sqrt{ }$ & $\sqrt{ }$ & $\sqrt{ }$ & & & & & & $\sqrt{ }$ & $\sqrt{ }$ & & $\sqrt{ }$ & 3.4 & 8.8 & 1 \\
\hline 30 & $\sqrt{ }$ & & $\sqrt{ }$ & $\sqrt{ }$ & $\sqrt{ }$ & & & & $\sqrt{ }$ & $\sqrt{ }$ & $\sqrt{ }$ & $\sqrt{ }$ & - & - & - \\
\hline 31 & $\sqrt{ }$ & $\sqrt{ }$ & $-\mathrm{ve}$ & & & & & & & $\sqrt{ }$ & $\sqrt{ }$ & $\sqrt{ }$ & 2.3 & 3.2 & 2 \\
\hline 32 & $\sqrt{ }$ & & & & & $\sqrt{ }$ & & & $\sqrt{ }$ & $\sqrt{ }$ & $\sqrt{ }$ & $\checkmark$ & - & - & - \\
\hline 33 & & $\sqrt{ }$ & $\sqrt{ }$ & & & & & & $\sqrt{ }$ & $\sqrt{ }$ & $\sqrt{ }$ & $\checkmark$ & -2.0 & 0.9 & 1 \\
\hline 34 & $\sqrt{ }$ & $\sqrt{ }$ & & $\sqrt{ }$ & $\sqrt{ }$ & $\sqrt{ }$ & & & $\sqrt{ }$ & $\sqrt{ }$ & $\sqrt{ }$ & $\sqrt{ }$ & - & - & - \\
\hline 35 & $\sqrt{ }$ & $\sqrt{ }$ & $\sqrt{ }$ & & & & & & & $\sqrt{ }$ & $\sqrt{ }$ & $\sqrt{ }$ & 2.1 & 8.4 & 1 \\
\hline 36 & $\sqrt{ }$ & & $\sqrt{ }$ & $\sqrt{ }$ & & & & & $\sqrt{ }$ & $\sqrt{ }$ & & $\sqrt{ }$ & - & - & - \\
\hline 37 & $\sqrt{ }$ & & & $\sqrt{ }$ & & & & & $\sqrt{ }$ & $\sqrt{ }$ & $\sqrt{ }$ & $\sqrt{ }$ & - & - & - \\
\hline 38 & $\sqrt{ }$ & $\sqrt{ }$ & $\sqrt{ }$ & $\checkmark$ & $\sqrt{ }$ & & $\checkmark$ & & $\sqrt{ }$ & $\sqrt{ }$ & $\checkmark$ & $\checkmark$ & $=$ & - & - \\
\hline 39 & $\sqrt{ }$ & $\sqrt{ }$ & $\checkmark$ & $\sqrt{ }$ & $\sqrt{ }$ & $\sqrt{ }$ & $\sqrt{ }$ & & $\sqrt{ }$ & $\sqrt{ }$ & $\sqrt{ }$ & $\sqrt{ }$ & $=$ & - & - \\
\hline 40 & $\sqrt{ }$ & & & $\sqrt{ }$ & & & & & & $\sqrt{ }$ & & $\sqrt{ }$ & 1.1 & 2.1 & 1 \\
\hline 41 & $\sqrt{ }$ & $\sqrt{ }$ & & & & & & & $\sqrt{ }$ & & $\sqrt{ }$ & $\sqrt{ }$ & - & - & - \\
\hline 42 & $\sqrt{ }$ & $\sqrt{ }$ & & & $\sqrt{ }$ & & $\sqrt{ }$ & & & $\sqrt{ }$ & $\sqrt{ }$ & $\checkmark$ & - & - & - \\
\hline 43 & $\sqrt{ }$ & $\sqrt{ }$ & & & $\sqrt{ }$ & & $\sqrt{ }$ & & $\sqrt{ }$ & & $\sqrt{ }$ & $\sqrt{ }$ & - & - & - \\
\hline 44 & $\sqrt{ }$ & $\sqrt{ }$ & $\sqrt{ }$ & $\sqrt{ }$ & & & $\sqrt{ }$ & & $\sqrt{ }$ & $\sqrt{ }$ & $\sqrt{ }$ & $\sqrt{ }$ & 0.3 & -5.8 & 1 \\
\hline 45 & $\sqrt{ }$ & $\sqrt{ }$ & & & $\sqrt{ }$ & & & & & & $\sqrt{ }$ & $\sqrt{ }$ & - & - & - \\
\hline 46 & $\sqrt{ }$ & & & & $\sqrt{ }$ & $\sqrt{ }$ & & & & $\sqrt{ }$ & $\sqrt{ }$ & $\sqrt{ }$ & - & - & - \\
\hline 47 & $\sqrt{ }$ & & $\sqrt{ }$ & $\sqrt{ }$ & $\sqrt{ }$ & $\sqrt{ }$ & & & $\sqrt{ }$ & $\sqrt{ }$ & $\sqrt{ }$ & $\sqrt{ }$ & - & - & - \\
\hline 48 & $\sqrt{ }$ & $\sqrt{ }$ & & & & & $\sqrt{ }$ & & & & $\sqrt{ }$ & $\checkmark$ & - & - & - \\
\hline 49 & $\sqrt{ }$ & $\sqrt{ }$ & $\sqrt{ }$ & $\sqrt{ }$ & & & & $\sqrt{ }$ & $\sqrt{ }$ & $\checkmark$ & $\sqrt{ }$ & $\checkmark$ & 1.0 & -2.6 & 2 \\
\hline 50 & & & $\sqrt{ }$ & & & & & & $\sqrt{ }$ & $\sqrt{ }$ & $\checkmark$ & $\sqrt{ }$ & 2.0 & 13.0 & 1 \\
\hline
\end{tabular}


Table 62: Model's change in VTR prediction for worksites with Transit share between $25 \%$ to $35 \%$ and Vehicle Trip Rate between 50 to 60

\begin{tabular}{|c|c|c|c|c|c|c|c|c|c|c|c|c|c|c|c|}
\hline $\begin{array}{c}\text { Sr. } \\
\text { No. }\end{array}$ & F\&A & ONS & CTB & FI N & CWW & FLEX & TELE & PMT & NONF & GRH & RSMP & MRKT & $\begin{array}{c}\text { Trar } \\
\text { [0. } \\
\text { VTR }\end{array}$ & $\begin{array}{l}\text { nsit Sha } \\
35-0.45 \\
-[60-5\end{array}$ & \\
\hline & & & & & & & & & & & & & Predicted & Actual & No.Ex \\
\hline 1 & $\sqrt{ }$ & $\sqrt{ }$ & $\sqrt{ }$ & $\sqrt{ }$ & & & & & $\sqrt{ }$ & $\sqrt{ }$ & $\sqrt{ }$ & $\sqrt{ }$ & -1.8 & 1.8 & 3 \\
\hline 2 & $\sqrt{ }$ & $\sqrt{ }$ & $\sqrt{ }$ & $\sqrt{ }$ & & $\sqrt{ }$ & & & $\sqrt{ }$ & $\sqrt{ }$ & $\sqrt{ }$ & $\sqrt{ }$ & - & - & - \\
\hline 3 & $\sqrt{ }$ & $\sqrt{ }$ & $\sqrt{ }$ & & & & & & $\sqrt{ }$ & $\sqrt{ }$ & $\sqrt{ }$ & $\sqrt{ }$ & -2.1 & -2.0 & 1 \\
\hline 4 & $\sqrt{ }$ & & $\sqrt{ }$ & $\sqrt{ }$ & & & & & $\sqrt{ }$ & $\sqrt{ }$ & $\sqrt{ }$ & $\sqrt{ }$ & - & - & - \\
\hline 5 & $\sqrt{ }$ & $\sqrt{ }$ & & & & & & & $\sqrt{ }$ & $\sqrt{ }$ & $\sqrt{ }$ & $\sqrt{ }$ & - & - & - \\
\hline 6 & & $\sqrt{ }$ & $\sqrt{ }$ & $\sqrt{ }$ & & & & & $\sqrt{ }$ & $\sqrt{ }$ & $\sqrt{ }$ & $\sqrt{ }$ & - & - & - \\
\hline 7 & $\sqrt{ }$ & $\sqrt{ }$ & & & & $\sqrt{ }$ & & & $\sqrt{ }$ & $\sqrt{ }$ & $\sqrt{ }$ & $\sqrt{ }$ & - & - & - \\
\hline 8 & $\sqrt{ }$ & $\sqrt{ }$ & $\sqrt{ }$ & $\sqrt{ }$ & $\sqrt{ }$ & & & & $\sqrt{ }$ & $\sqrt{ }$ & $\sqrt{ }$ & $\sqrt{ }$ & - & - & - \\
\hline 9 & $\sqrt{ }$ & & $\sqrt{ }$ & & & & & & $\sqrt{ }$ & $\sqrt{ }$ & $\sqrt{ }$ & $\sqrt{ }$ & - & - & - \\
\hline 10 & $\sqrt{ }$ & $\sqrt{ }$ & & & $\sqrt{ }$ & & & & $\sqrt{ }$ & $\sqrt{ }$ & $\sqrt{ }$ & $\sqrt{ }$ & - & - & - \\
\hline 11 & $\sqrt{ }$ & & $\sqrt{ }$ & $\sqrt{ }$ & & & & & & $\sqrt{ }$ & $\sqrt{ }$ & $\sqrt{ }$ & - & - & - \\
\hline 12 & $\sqrt{ }$ & & & & & & & & & $\sqrt{ }$ & $\sqrt{ }$ & $\sqrt{ }$ & - & - & - \\
\hline 13 & $\sqrt{ }$ & $\sqrt{ }$ & $\sqrt{ }$ & & & $\sqrt{ }$ & & & $\sqrt{ }$ & $\sqrt{ }$ & $\sqrt{ }$ & $\sqrt{ }$ & - & - & - \\
\hline 14 & $\sqrt{ }$ & $\sqrt{ }$ & $\sqrt{ }$ & $\sqrt{ }$ & & & & & & $\sqrt{ }$ & $\sqrt{ }$ & $\sqrt{ }$ & - & - & - \\
\hline 15 & $\sqrt{ }$ & 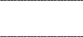 & 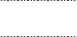 & & & & & & $\sqrt{ }$ & $\sqrt{ }$ & $\sqrt{ }$ & $\sqrt{ }$ & 1.4 & 3.4 & 1 \\
\hline 16 & $\sqrt{ }$ & $\sqrt{ }$ & $\sqrt{ }$ & $\sqrt{ }$ & $\sqrt{ }$ & $\sqrt{ }$ & & & $\sqrt{ }$ & $\sqrt{ }$ & $\sqrt{ }$ & $\sqrt{ }$ & - & - & - \\
\hline 17 & $\sqrt{ }$ & $\sqrt{ }$ & $\sqrt{ }$ & $\sqrt{ }$ & & & & & $\sqrt{ }$ & $\sqrt{ }$ & & $\sqrt{ }$ & - & - & - \\
\hline 18 & $\sqrt{ }$ & $\sqrt{ }$ & $\sqrt{ }$ & & $\sqrt{ }$ & & & & $\sqrt{ }$ & $\sqrt{ }$ & $\sqrt{ }$ & $\sqrt{ }$ & - & - & - \\
\hline 19 & $\sqrt{ }$ & & $\sqrt{ }$ & $\sqrt{ }$ & & $\sqrt{ }$ & & & $\sqrt{ }$ & $\sqrt{ }$ & $\sqrt{ }$ & $\sqrt{ }$ & - & - & - \\
\hline 20 & & & $\sqrt{ }$ & $\sqrt{ }$ & & & & & $\sqrt{ }$ & $\sqrt{ }$ & $\sqrt{ }$ & $\sqrt{ }$ & - & - & - \\
\hline 21 & $\sqrt{ }$ & $\sqrt{ }$ & & & $\sqrt{ }$ & & $\sqrt{ }$ & & $\sqrt{ }$ & $\sqrt{ }$ & $\sqrt{ }$ & $\sqrt{ }$ & - & - & - \\
\hline 22 & $\sqrt{ }$ & & $\sqrt{ }$ & & & $\sqrt{ }$ & & & $\sqrt{ }$ & $\sqrt{ }$ & $\sqrt{ }$ & $\sqrt{ }$ & - & - & - \\
\hline 23 & $\sqrt{ }$ & & & & & & & & & & $\sqrt{ }$ & $\sqrt{ }$ & - & - & - \\
\hline 24 & $\sqrt{ }$ & $\sqrt{ }$ & & $\sqrt{ }$ & & & & & $\sqrt{ }$ & $\sqrt{ }$ & $\sqrt{ }$ & $\sqrt{ }$ & - & - & - \\
\hline 25 & $\sqrt{ }$ & $\sqrt{ }$ & $\sqrt{ }$ & & $\sqrt{ }$ & $\sqrt{ }$ & & & $\sqrt{ }$ & $\sqrt{ }$ & $\sqrt{ }$ & $\sqrt{ }$ & - & - & - \\
\hline 26 & $\sqrt{ }$ & $\sqrt{ }$ & $\sqrt{ }$ & $\sqrt{ }$ & & $\sqrt{ }$ & & & & $\sqrt{ }$ & $\sqrt{ }$ & $\sqrt{ }$ & - & - & - \\
\hline 27 & $\sqrt{ }$ & $\sqrt{ }$ & 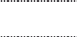 & & & & & & & & $\sqrt{ }$ & $\sqrt{ }$ & - & - & - \\
\hline 28 & $\sqrt{ }$ & $\sqrt{ }$ & $\sqrt{ }$ & & $\sqrt{ }$ & & $\sqrt{ }$ & & $\sqrt{ }$ & $\sqrt{ }$ & $\sqrt{ }$ & $\sqrt{ }$ & - & - & - \\
\hline 29 & $\sqrt{ }$ & $\sqrt{ }$ & $\sqrt{ }$ & & & & & & $\sqrt{ }$ & $\sqrt{ }$ & - & $\sqrt{ }$ & -1.6 & 8.5 & 1 \\
\hline 30 & $\sqrt{ }$ & . & $\sqrt{ }$ & $\sqrt{ }$ & $\sqrt{ }$ & & & & $\sqrt{ }$ & $\sqrt{ }$ & $\sqrt{ }$ & $\sqrt{ }$ & - & - & - \\
\hline 31 & $\sqrt{ }$ & $\sqrt{ }$ & & & & & & & & $\sqrt{ }$ & $\sqrt{ }$ & $\sqrt{ }$ & -0.9 & 8.3 & 1 \\
\hline 32 & $\sqrt{ }$ & . & & & & $\sqrt{ }$ & & & $\sqrt{ }$ & $\sqrt{ }$ & $\sqrt{ }$ & $\sqrt{ }$ & - & - & - \\
\hline 33 & & $\sqrt{ }$ & $\sqrt{ }$ & & & & & & $\sqrt{ }$ & $\sqrt{ }$ & $\sqrt{ }$ & $\sqrt{ }$ & - & - & - \\
\hline 34 & $\sqrt{ }$ & $\sqrt{ }$ & & $\sqrt{ }$ & $\sqrt{ }$ & $\sqrt{ }$ & & & $\sqrt{ }$ & $\sqrt{ }$ & $\sqrt{ }$ & $\sqrt{ }$ & - & - & - \\
\hline 35 & $\sqrt{ }$ & $\sqrt{ }$ & $\sqrt{ }$ & & & & & & & $\sqrt{ }$ & $\sqrt{ }$ & $\sqrt{ }$ & - & - & - \\
\hline 36 & $\sqrt{ }$ & & $\sqrt{ }$ & $\sqrt{ }$ & & & & & $\sqrt{ }$ & $\sqrt{ }$ & - & $\sqrt{ }$ & - & - & - \\
\hline 37 & $\sqrt{ }$ & & & $\sqrt{ }$ & & & & & $\sqrt{ }$ & $\sqrt{ }$ & $\sqrt{ }$ & $\sqrt{ }$ & - & - & - \\
\hline 38 & $\sqrt{ }$ & $\sqrt{ }$ & $\sqrt{ }$ & $\sqrt{ }$ & $\sqrt{ }$ & & $\sqrt{ }$ & & $\sqrt{ }$ & $\sqrt{ }$ & $\sqrt{ }$ & $\sqrt{ }$ & - & - & - \\
\hline 39 & $\sqrt{ }$ & $\sqrt{ }$ & $\sqrt{ }$ & $\sqrt{ }$ & $\sqrt{ }$ & $\sqrt{ }$ & $\sqrt{ }$ & & $\sqrt{ }$ & $\sqrt{ }$ & $\sqrt{ }$ & $\sqrt{ }$ & - & - & - \\
\hline 40 & $\sqrt{ }$ & & & $\sqrt{ }$ & & & & & & $\sqrt{ }$ & & $\sqrt{ }$ & - & - & - \\
\hline 41 & $\sqrt{ }$ & $\sqrt{ }$ & & & & & & & $\sqrt{ }$ & & $\sqrt{ }$ & $\sqrt{ }$ & - & - & - \\
\hline 42 & $\sqrt{ }$ & $\sqrt{ }$ & & & $\sqrt{ }$ & & $\sqrt{ }$ & & & $\sqrt{ }$ & $\sqrt{ }$ & $\sqrt{ }$ & - & - & - \\
\hline 43 & $\sqrt{ }$ & $\sqrt{ }$ & & & $\sqrt{ }$ & & $\sqrt{ }$ & & $\sqrt{ }$ & & $\sqrt{ }$ & $\sqrt{ }$ & - & - & - \\
\hline 44 & $\sqrt{ }$ & $\sqrt{ }$ & $\sqrt{ }$ & $\sqrt{ }$ & & & $\sqrt{ }$ & & $\sqrt{ }$ & $\sqrt{ }$ & $\sqrt{ }$ & $\sqrt{ }$ & - & - & - \\
\hline 45 & $\sqrt{ }$ & $\sqrt{ }$ & & & $\sqrt{ }$ & & & & & & $\sqrt{ }$ & $\sqrt{ }$ & - & - & - \\
\hline 46 & $\sqrt{ }$ & & & & $\sqrt{ }$ & $\sqrt{ }$ & & & & $\sqrt{ }$ & $\sqrt{ }$ & $\sqrt{ }$ & - & - & - \\
\hline 47 & $\sqrt{ }$ & & $\sqrt{ }$ & $\sqrt{ }$ & $\sqrt{ }$ & $\sqrt{ }$ & & & $\sqrt{ }$ & $\sqrt{ }$ & $\sqrt{ }$ & $\sqrt{ }$ & - & - & - \\
\hline 48 & $\sqrt{ }$ & $\sqrt{ }$ & & & & & $\sqrt{ }$ & & & & $\sqrt{ }$ & $\sqrt{ }$ & - & - & - \\
\hline 49 & $\sqrt{ }$ & $\sqrt{ }$ & $\sqrt{ }$ & $\sqrt{ }$ & & & & $\sqrt{ }$ & $\sqrt{ }$ & $\sqrt{ }$ & $\sqrt{ }$ & $\sqrt{ }$ & - & - & - \\
\hline 50 & & & $\sqrt{ }$ & & & & & & $\sqrt{ }$ & $\sqrt{ }$ & $\sqrt{ }$ & $\sqrt{ }$ & - & - & - \\
\hline
\end{tabular}




\section{SUMMARY}

This project was undertaken with purpose of simplifying the transportation management process for all parties in the development and planning process for both residential and commercial enterprises. The software model developed from this project provides a valuable and interactive tool for anyone involved in the decision-making process for new developments and transportation programs. This software allows for precise prediction to the extent each incentive, disincentive, or program has an effect on vehicle trip rate.

This software model takes much of the guesswork out of planning developmental impact and roadway level of service. The user of the software can enter data for several variables, such as the number of employees, the shares of different commuting modes used by the employees, incentives offered, disincentives charged, etc. The learned model built on the data obtained from various sources culled from years of longitudinal research, returns a predictive answer as to the amount the vehicle trips that will be reduced or increased.

This project used several thousand worksite trip reduction plans to build the model. The data came from three urban areas in the United States: Los Angeles, Tucson, and Washington State that have had trip reduction requirements on employers for many years. Employers were required to submit plans to reach a particular objective such as a reduction in the levels of single occupant vehicle (SOV) use. The data consisted of worksite modal characteristics aggregated at the employer level and a listing of incentives and amenities offered by employers. The Los Angeles data contained the largest data set data, with the Tucson, Arizona and Washington state data sets being considerably smaller. Data quality control problems reduced the size of the data in each area and eliminated or restricted some potentially useful variables (e.g., dollar values of some incentives). For performance evaluation the datasets were divided in two disjoint sets 'training/testing set' which was used to build the models and 'validation set' which was used as an unseen data to evaluated the models.

The dependent variable chosen was the change in vehicle trip rate (VTR) (e.g., reduction of 4.5 vehicles per 100 employees). VTR correlates closely with the goals of TDM -- reduce trips, decrease air pollution, decrease the need for parking -- and is generally proportional to the desired result. Alternative dependent variables such as SOV share or average vehicle ridership (AVR) have disadvantages. SOV share misses the benefits of moving from one non-SOV mode to another where the switch may actual reducing traffic but not affect SOV share (e.g., carpool to transit). The reduction in vehicle trips is distorted when using AVR as the dependent variable due to the non-liner relationship between AVR and vehicle trips. 
For example, increasing AVR by 0.25 from 1.10 to 1.35 persons per vehicle would require a reduction in 17 vehicle trips per 100 people. The same increase (0.25) for a worksite with an AVR of 1.50 to 1.75 would only require a reduction in 9 vehicle trips per 100 people.

Two approaches were used for the model building process: linear statistical regression models and non-linear neural networks. The linear statistical regression models were used as a benchmark for the validity and accuracy of the neural net models. The linear statistical regression models minimize the sum of the error between the real and predicted data, learning simple linear relationships between the worksite characteristics, incentives and the dependant variable 'change in VTR', while the neural networks learn more complex non-linear relationships. Sometimes linear regression methods were used to determine which variables the neural net would use to build its models.

Several phases were followed to build the models. Models were built for each of the three datasets using a variety of approaches of handling the data, including variable selection, grouping of incentives, and the treatment of outliers. Models were also built after combining the data from the three urban areas into a single dataset. Under the assumption the transportation industry was most interested in a model that predicted when large reductions could be achieved, the model performance objective was focused on predicting the change equally well across the range of the changes in VTR.

\section{Los Angeles}

\section{Phase I}

The Los Angeles dataset consisted of 25,459 total records. A first attempt to build neural network models using all the variables present in the data resulted in very poor performance. So, different linear regression modeling approaches were used to select different variables sets on which neural network models were built. The neural network model built using stepwise method variable set was able to get better results on all performance measures.

\section{Phase II}

Next, the Los Angeles data was over-sampled in some bins and under-sampled in others in order to improve the accuracy on moderate range of change in VTR. In addition, this data was added with the costs associated with each incentive, and a stepwise neural net model was built. It was concluded that the over-sampling biased the model towards predicting more negative changes in VTR with reduction in overall accuracy.

\section{Phase III}

Later it was discovered that some of the records in the data sets had not a single incentives implemented. It was felt that any worksite which goes 
from having some incentives in one year to no incentive the next year and again having some incentive the next to next year, was missing the information regarding the incentive plans for the intermediate year. So it was decided that these records be removed as they might influence the model in some wrong direction. The cleaned dataset now contained 18,140 records in total. Also, it was felt that a simple model which was not significantly worse than the best complex model would be more preferred due to its simplicity. So all of the individual incentives were replaced by the grouped incentives and news models were built. The two best models obtained from this model-building session were the ones constructed using the neural network: one built using stepwise regression variables on incentives and costs and the other one built on grouped incentives. Both models got $16.92 \%$ accuracy on full range of change in VTR, with comparable accuracies on moderate range of change in VTR and R-square values.

\section{Phase IV}

Finally, it was felt that by removing the records with very large changes in VTR (which might not be the effect of incentives offered) might improve models performance. So a normal distribution of the data set was produced and all records which were outside of three distributions above or below the mean were removed from the set. In effect 790 records were dropped from the validation set and 7,432 from the training set. Three models were built with variables based on previously constructed models. However, the best model, the one built from stepwise regression on incentives with costs added was still lacking in accuracies when compared to the models from previous phases.

\section{Recommended Los Angeles Model}

The two candidate models were the ones built on data with records with 'no incentives' removed using the stepwise regression variables on incentives and costs and the other built on grouped incentives. There was no significant difference between their accuracy measures of the two models. So the simple model built on data with grouped incentives was regarded as the recommended Los Angeles Model.

\section{Tucson}

\section{Phase I}

The Tucson data set consisted of 1,121 total records. The models built with variable selection did not select many of the incentives variables in predicting change in VTR and so were considered unsuitable. The neural network model built with no variable selection got the best 'bin classification on full range of change in VTR' of $20.54 \%, 16.60 \%$ accuracy on moderate range of change in VTR and the R-square value of 0.022 . 
To reduce the complexity of the previous models, all of the individual incentives were replaced with grouped incentives. The neural network model built with no variable selection seemed to over-fit the training data and got poor results on validation data. While the linear regression model was better than the neural network model it was still worse than the previously built neural network model on individual ungrouped incentives

\section{Phase II}

In this phase, the Tucson data was over-sampled to boost the accuracy in the moderate range of change in VTR. A neural network model built with no variable selection which got $18.75 \%$ accuracy on full range of change in VTR, $12.77 \%$ accuracy on moderate range of change in VTR and an $\mathrm{R}$-square value of 0.036 gave balanced predictions.

A better model on over-sampled grouped incentive data was the neural network model having $16.96 \%$ accuracy on full range of change in VTR, $14.86 \%$ accuracy on moderate range of change in VTR with 0.007 R-square.

\section{Recommended Tucson Model}

In the end, two neural network models with no variable selection were front runners for the best model: one was built on full sample data with individual incentives, and the other was built on the over-sampled grouped incentives data. The winner was evidently the neural network model built on individual incentives on full sample data. 


\section{Washington}

\section{Phase I}

The Washington data set consisted of 1,414 total records. Since some variables in the data might not be readily available to employers or program coordinators who might be using the software, employee's preferences towards incentives and the type of work variables were removed from the data. In the study it was found that the employee's preferences towards incentive variables did indeed influence the change in VTR. However, since these variables may not be available to future software users, they were only used to study their impact. The two models which came out as front runners were the linear forced enter regression model and the neural network model both built on all variables except for preferences.

To reduce the complexity of the models, all of the individual incentives were replaced with grouped incentives and simple neural network with no variable selection and a linear forced enter regression model were built. The neural network model built showed a better distribution of accuracy in the moderate range of change in VTR than the linear regression forced enter model. The scatter plots also showed that the predictions of the neural network model built with all grouped variables were much closer to the actual values.

\section{Phase II}

Since better accuracies were desired on the moderate range of change in VTR, an over-sampling of the Washington data was done. Again the models with employee's preferences towards incentives came out better restating their importance in predicting changes in VTR. But, since the preference variable information might not readily accessible to everyone, the models built without preference variables were considered for final analysis. The forced enter regression models from this model-building session came up to be the best, but still it performed worse than its equivalent model on original full sample data.

Two more models were built with grouped incentives over-sampled data. The better model was the neural network built with no variable selection as it seemed to be less biased than the linear regression model.

\section{Recommended Washington Model}

As stated in the previous sections, the neural network models built in both phases I and II data including employee's preferences towards incentives obtained much better accuracies than the models built without them. However, given that this information might not be available to future model builders, only models without preferences were considered.

The two models that were considered as the best on the Washington data were the forced enter linear regression model built on the individual 
incentive full sample data and the neural network model built on grouped incentive over-sampled data. The models were so close that a cross-tab of positive/negative actual/predicted changes in VTR was set-up to decide. The most important factor of the best model was its ability to predict correct negative changes in VTR. So it was desirable to improve recall which captured the completeness of the model at predicting negative changes in VTR without sacrificing precision which captured the correctness of the model at predicting negative changes in VTR. The f-value weighted these two measures equally (with $\beta=1$ ) to give one performance measure. The forced enter linear regression had the best $f$-value on validation set so was considered the recommended model for the Washington data set.

\section{Combined Data Models}

All of the incentive data from each of the three data sets was combined to build a larger, generalized model, based on the information of all three areas. Some of variables had to be collapsed into one group variable in order to ensure uniformity of the incentives.

\section{Phase I}

The training data from all three cities was combined into a single training set, while the validation sets for each of the cities were left intact for testing of the combination training sets. Neural net with no variables selected models and forced enter linear regression models were built on data containing only 23 variables. The models were evaluated on the three validation sets and then compared with best independent model from three locations. It was found that the neural net model built on the combined data with no variable selection was able to get better 'bin classification accuracy on moderate range of change in VTR' on the Los Angeles validation set as compared to the recommended independent Los Angeles model built on grouped data. The combined data neural net model also was able to improve bin classification for the recommended independent Tucson model built on over-sampled grouped incentive data. The Washington data set and recommended model did not have improved accuracy with the combination neural net model.

It was concluded from this neural network model with no variable selection that by adding more data from the other datasets to Los Angeles data helped in improving the accuracy of the Los Angeles model, and therefore became the recommended model for the Los Angeles data. However, the Tucson data, while increasing accuracy on moderate range of change in VTR at expense of accuracy on full range, became more biased to predicting negative VTR changes and the Washington data actually showed a decrease in accuracy with the Phase I combination neural net model. 


\section{Phase II}

To deal with the combined data neural network model's problems with the Washington and Tucson data sets, the three training sets of data were sampled equally to get equally combined training data set. This data set contains 2,018 records from each data set. A neural network and a forced enter linear regression model were built on this new equally sampled data. The equally sampled combined data set models still could not improve the accuracy on the Washington validation set. The equally combined data neural network model was able to improve accuracy on Tucson validation set on moderate range of change in VTR with reduced R-square value. But still it was difficult to state that it was the best model for Tucson data. So a cross-tab was done, and the neural network model built on the equally sampled combined data resulted in better Recall F-value on the Tucson validation set and therefore became the recommended model for the Tucson data.

\section{Best Generalized Model}

Overall, the best generalized model for any location is the neural net model built on equally sampled data based on the three performance measures described earlier.

Table 63 Performance Measures for Best Generalized Model

\begin{tabular}{|l|l|l|l|l|}
\hline & LA Validation & $\begin{array}{l}\text { Washington } \\
\text { Validation }\end{array}$ & $\begin{array}{l}\text { Tucson } \\
\text { Validation }\end{array}$ & Training Set \\
\hline $\begin{array}{l}\text { Bin Classification } \\
\text { Accuracy on moderate } \\
\text { range of change in VTR } \\
\text { (i.e. bins a2 to a5) }\end{array}$ & $23.20 \%$ & $14.62 \%$ & $25.32 \%$ & $29.24 \%$ \\
\hline $\begin{array}{l}\text { Bin Classification } \\
\text { Accuracy on full range } \\
\text { of change in VTR (i.e. } \\
\text { all bins) }\end{array}$ & $15.78 \%$ & $12.50 \%$ & $20.54 \%$ & $21.09 \%$ \\
\hline R-square & 0.075 & 0.011 & 0.013 & 0.215 \\
\hline
\end{tabular}

This is the model at http://www.nctr.usf.edu/worksite. 


\section{I. CONCLUSION}

No single variable selection technique, data handling method, or modeling approach yielded the best-fitting model for all urban areas. In many cases, there was no significant performance difference between the top models, so the recommended model for some dataset had to be decided by using the F-value measure, which incorporated two other metrics: Recall which gave a measure of the completeness of the model, and Precision which gave a measure of the correctness of the model.

The best model for each city also was not the model that used data only from that city. Before combining the Los Angeles data set with those from the other two areas, the preferred model was the one built on the grouped incentives data with records with 'no incentives' removed. But after combining the datasets, the neural network model built with no variable selection performed better for Los Angeles than the model built with only data from Los Angeles. Also for Tucson data, a neural network model built on the equally sampled (i.e., each dataset contributes equally) data performed better than the previously selected neural network model built on the full sample (i.e., all valid records from three datasets) with ungrouped incentives data. The best model for the Washington data was the linear forced enter regression model built on full sample with ungrouped incentive data.

The generalized models for any urban area were built on the combined training datasets and equally sampled training datasets. The models built using equally sampled datasets were the ones which were not biased towards the any dataset. So the best generalized model for any location is the neural net model built on equally sampled data which is the version deployed at http://www.nctr.usf.edu/worksite.

Overall, the neural net models performed better than the linear regression models. This might be due to the ability of the neural network program to move beyond simple linear regression, which tries to minimize the error between the predicted and actual data sets. The neural network models in many situations were able to learn the non-linear relationships among various combinations of strategies. There were some neural network models which performed worse than the linear models. This might be due to the over-fitting of the training data and reducing the neural net's power to generalize over unseen validation data.

Quality control issues with the provided datasets affected the model building process. In the case of the Los Angeles data, there were many worksites for which some incentives were available in one year, then not a single incentive was shown in the following year, only to have incentives "reappear" the next plan cycle. These unexplained gaps in reporting can 
affect the ability of the model to estimate the impact of a particular incentive.

Another data problem encountered was the use of different units of measurement across programs. For example, the transit subsidy values were reported ranging from $\$ 0.20$ to $\$ 3,000$. Though employers were to report these values as "cost per employee per month", the lower figure basis appears to be along the lines of "cost per employee per trip" and the upper figure might be the "total cost per employee using the mode per year". The difficulty is there is no way of telling from the data. Also it was found that the incentives had differences in their definition across different datasets which introduced error into the results. This problem made modelbuilding a complex task when trying to condense and collapse all of the similar variables into one.

The aggregate nature of the data loses the ability to explain whether the change in mode behavior was influenced by the programs or changes in the workforce or other exogenous variables. While hundreds of thousands of employee data, including employee's preferences to particular options, were available from the State of Washington dataset, there was no identifier to track individual changes in behavior over time. The other two datasets did not have any comparable data to Washington's individual survey responses. Access to such disaggregate data could improve the ability of a model to track behavior changes over time based on changes to worksite incentives, amenities and programs. As suggested in the Future Work section, the development of a nationwide database which would include employee's preferences for incentives, similar to those used in the State of Washington data. 


\section{FUTURE WORK}

Given the common interest shared by the public sector and worksites in assessing the relative effectiveness of worksite trip reduction program, future work should begin by improving the quality of the data already being collected. Quality can be improved by adopting standard definitions and common terminology. Common terms will contribute to an expanded dataset by making the data compatible with other data from other parts of the country. This approach could be facilitated by the creation of a centralized database.

Adhering to quality control procedures also could add more explanatory power to the data, especially as it relates to the value of financial incentives. Improving the quality of information that already exists can help worksites more cost-effectively deliver vehicle trip reduction programs.

The data used in this model-building approach was aggregated to the worksite level prior by the employer. This aggregate level detail does not allow for analysis to determine which individuals' travel behavior has changed. In order to get a real handle on what makes VTR increase or decrease and what causes people to choose alternative transportation options, attempts to control for the differences should be used. Access to disaggregate data collected over time can help establish a "test" and "control" group approach to control for differences, for example, in the composition of the workforce.

Access to the disaggregate data also would help track the long-term effects of the programs. The current project assesses the impacts between two time periods (usually separated by only one to two years). However, the cumulative effect of these programs over time is less understood (i.e., will the worksite experience a constant, variable, or exponential change in VTR over time as the programs diffuse within the workforce and move beyond the "early adopters"?) Diffusion is the process by which the trip reduction program offerings are communicated through certain channels over time among the employees at that location. While the collection of individual data may be difficult due to privacy and attrition issues, it is worth investigating the possibility of collecting this type of transportation behavior data to help develop sustainable transportation strategies and programs for the future. 